ENGINEERING CHANGE NOTICE

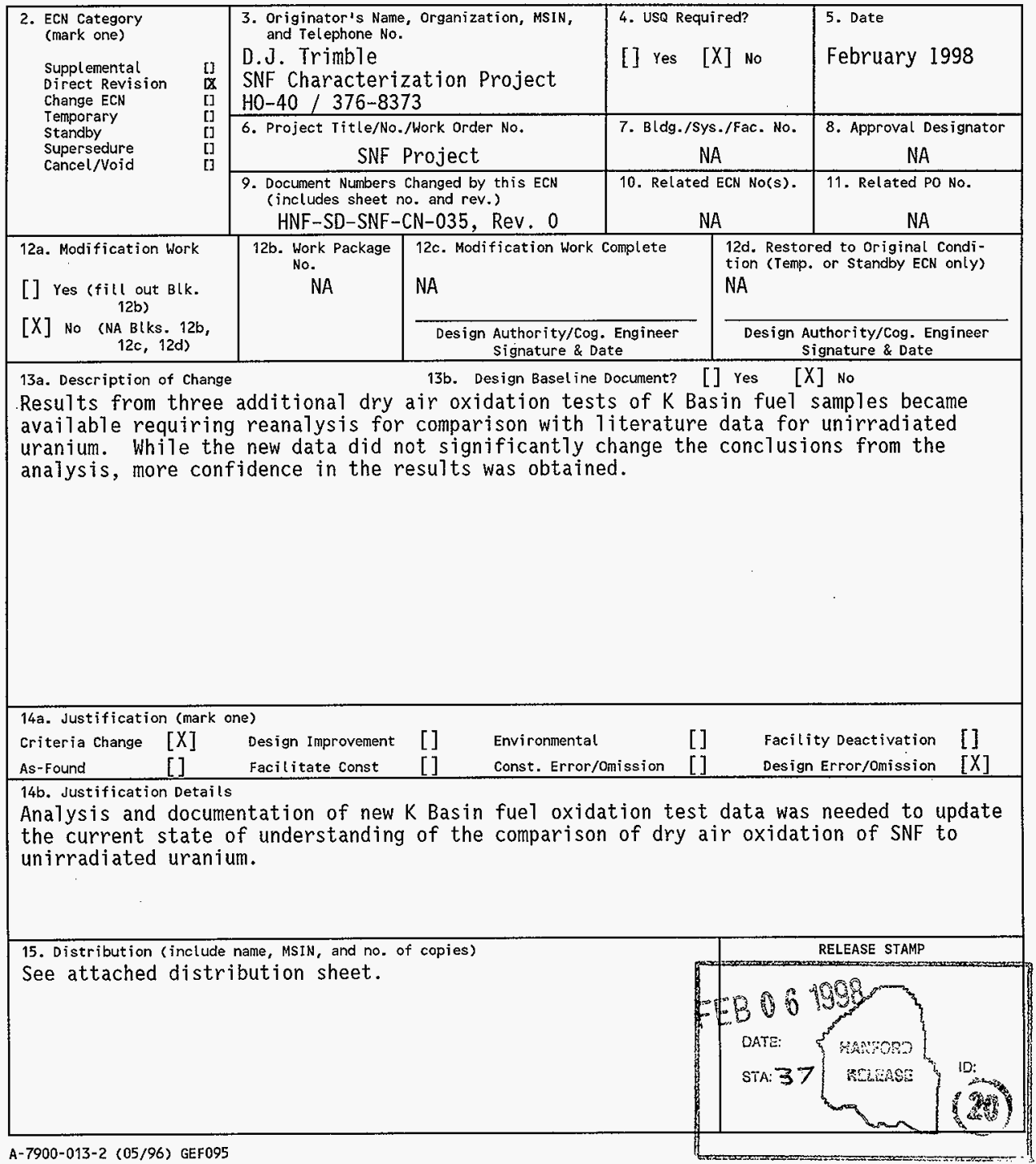




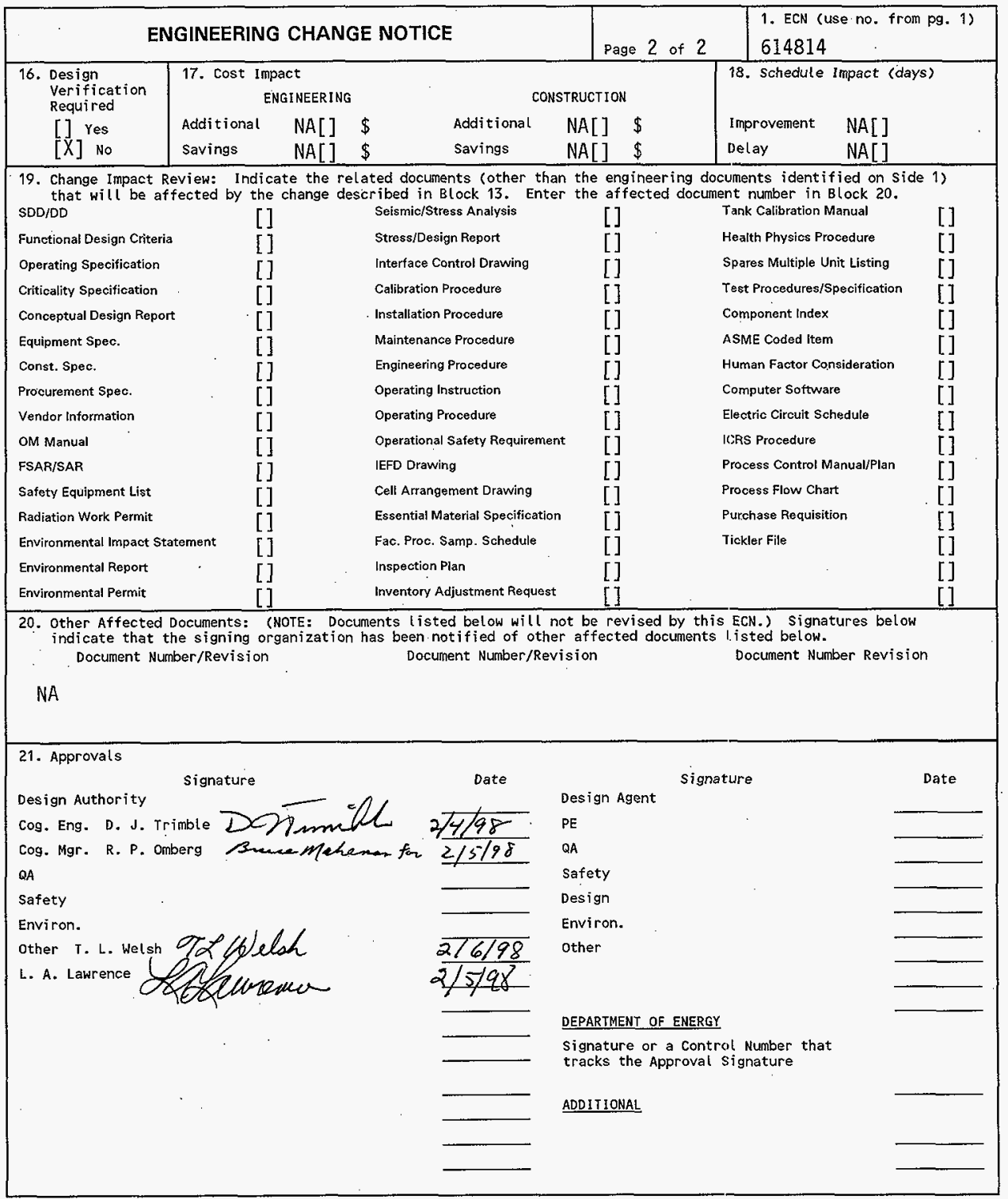




\title{
STATISTICAL ANALYSIS OF OXIDATION RATES FOR K BASIN FUEL IN DRY AIR
}

\author{
D. J. Trimble and T. L. Welsh* \\ Duke Engineering and Services Hanford, Richland, WA 99352 \\ U.S. Department of Energy Contract DE-AC06-96RL13200
}

\begin{tabular}{|c|c|c|}
\hline $\begin{array}{l}\text { EDT/ECN: } \\
\text { Org Code } \\
\text { B\&R Code }\end{array}$ & $\begin{array}{l}614814 \\
2 T 720 \\
\text { EW7040000 }\end{array}$ & $\begin{array}{l}\text { UC: } 2070 \\
\text { Charge Code: } \\
\text { Total Pages: }\end{array}$ \\
\hline
\end{tabular}

Key Words: K Basin, Spent Nuclear Fuel, N Reactor Fuel, Uranium, Oxidation, Dry Air

Abstract: Test data from oxidation of $K$ Basin fuel (SNF) samples in dry air were reviewed, and linear reaction rates were derived on a time-average basis. The derived rates were compared to literature data for unirradiated uranium in dry air using rate law of the form $\log ($ rate $)=a+b(1 / T)$. The analyses found differences between the SNF data and the Titerature data. Oxidation rate below $150^{\circ} \mathrm{C}$ was higher for $K$ Basin fuel than for unirradiated uranium.

*B\&W Protec, Inc.

TRADEMARK DISCLAIMER. Reference herein to any specific commercial product, process, or service by trade name, trademark, manufacturer, or otherwise, does not necessarily constitute or imply its endorsement, recomendation, or favoring by the United States Government or any agency thereof or its contractors or subcontractors.

Printed in the United States of America. To obtain copies of this document, contact: Document Control Services, P.O. Box 950, Mailstop H6-08, Richland WA 99352, Phone (509) 372-2420; Fax (509) 376-4989.
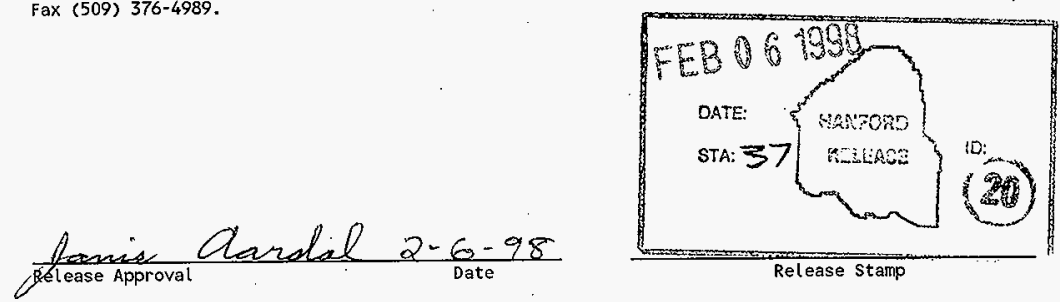
(2) Title

PSTATISTICAL ANALYSIS OF OXIDATION RATES FOR K BASIN FUEL IN DRY AIR

CHANGE CONTROL RECORD

(3) Revision (4) Description of Change - Replace, Add, and Delete Pages

(7) Initial Release, EDT 620798

0

1 RS
ECN 614814.

Complete rewrite presenting new data and revised analysis, results, discussion, and conclusions.
Authorized for Release

(5) Cog. Engr. (6) Cog. Mgr. Dạţe

D. J. Trimble $\quad$ R. P. Omberg 12/19/97

D. J. Trimble $\quad$ R. P. Omberg $2 / 6 / 98$

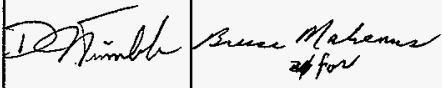


HNF-SD-SNF-CN-035, Rev . 1

\section{STATISTICAL ANALYSIS OF OXIDATION RATES}

FOR $K$ BASIN FUEL IN DRY AIR

D. J. Trimble

Duke Engineering \& Services Hanford, Inc.

T. L. Welsh

B\&W Protec

February 1998 
HNF-SD-SNF-CN-035, Rev. 1

\section{CONTENTS}

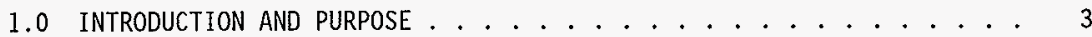

2.0 SUMMARY RESULTS . . . . . . . . . . . . . . . . . 5

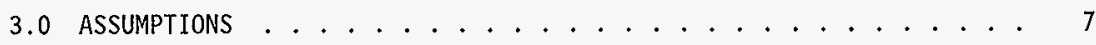

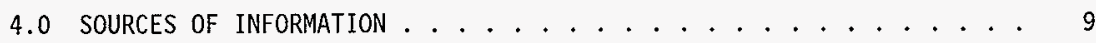

5.0 ANALYSIS OF DATA .............................. 11

5.1 OXIDATION RATE DATA FROM THE LITERATURE . . . . . . . . . 11

5.2 K BASIN FUEL TEST DATA . . . . . . 12

5.3 OXIDATION RATE CONSTANTS FROM K BASIN FUEL TESTS . . . . . 12

5.4 COMPARISON OF K BASIN FUEL OXIDATION RATES WITH LITERATURE DATA $\ldots \ldots \ldots$. . . . . . . . 16

5.5 REGRESSION ANALYSIS OF K BASIN FUEL DATA . . . . . . . 20

5.6 SUMMARY OBSERVATIONS AND DISCUSSION ............. 24

6.0 CONCLUSIONS ........................... 25

7.0 REFERENCES : . . . . . . . . . . . . . . . . . . . . 27

APPENDIX A DATA BASE FOR OXIDATION OF UNIRRADIATED URANIUM IN DRY AIR ............ . . 29

APPENDIX B WEIGHT GAIN DATA PLOTS WITH RATE ANALYSIS

DATA FROM OXIDATION TESTING OF $K$ BASIN

FUEL SAMPLES IN DRY AIR . . . . . . . . . . 33

APPENDIX $C$ WEIGHT GAIN DATA TABLES FROM OXIDATION TESTING

OF K BASIN FUEL SAMPLES IN DRY AIR ......... 


\section{LIST OF FIGURES}

1. Literature Data for Linear Oxidation of Unirradiated Uranium in Dry Air at Temperatures below $400{ }^{\circ} \mathrm{C} \ldots \ldots 11$

2. Data Plots for $K$ Basin Fuel Oxidation Test Runș Exhibiting Normal or Near Normal Behavior . . . . . . . . . 13

3. Thermo-Gravimetric Analysis Data Plots for K Basin Fuel Sample Runs at $144{ }^{\circ} \mathrm{C}$ and $150{ }^{\circ} \mathrm{C}$........... 14

4. Thermo-Gravimetric Analysis Data Plots for K Basin Fuel Sample Runs at $200^{\circ} \mathrm{C}$. . . . . . . . . . . . . . 14

5. Thermo-Gravimetric Analysis Data Plots for K Basin Fuel Sample Runs at $300^{\circ} \mathrm{C}$. . . . . . . . . . . 15

6. Thermo-Gravimetric Analysis Data Plots for K Basin Fuel Sample Runs at $350^{\circ} \mathrm{C}$ and $400{ }^{\circ} \mathrm{C} \ldots . . . . . . .215$

7. Oxidation Rates from $\mathrm{K}$ Basin Fue $\mathrm{T}$ Tests and Prediction Lines from Regression of Literature Data. . . . . . . . . . 19

8. Standardized Residuals from Regression of K Basin Fuel Oxidation Rate Data . . . . . . . . . . . . 20

9. Regression Lines for Dry Air Oxidation Rate Data from K Basin Fuel Tests ................ .

10. Comparison of Regressions of Oxidation Rate Data for Unirradiated Uranium and $K$ Basin Fue 1

\section{LIST OF TABLES}

1. Sample Data and Results from Thermo-Gravimetric Analysis

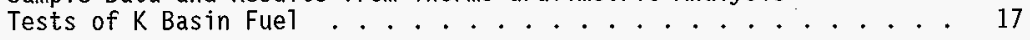

2. Data Groupings Used in Outlier Tests... . . . . . . . . . . 19

3. P-Values for Comparisons of Linear Regression Constants for Various Sets of Oxidation Rate Data ............. 
HNF-SD-SNF-CN-035, Rev. 1

\section{STATISTICAL ANALYSIS OF OXIDATION RATES FOR $K$ BASIN FUEL IN DRY AIR}

\subsection{INTRODUCTION AND PURPOSE}

Oxidation rate of irradiated $N$ Reactor fuel (SNF) which have been stored in the $K$ Basins (K Basin fuel) have been measured in support of the Spent Nuclear Fuel (SNF) Project. The measurements were made by Pacific Northwest National Laboratory (PNNL) using thermo-gravimetric analysis (TGA). Smal1 samples from damaged portions of K Basin fue 1 were oxidized in dry air at constant temperatures ranging from $69^{\circ} \mathrm{C}$ to $400^{\circ} \mathrm{C}$, and sample weight change was measured as a function of time. These measurements and an evaluation of the data was reported by Abrefah et a1. (1998).

This report documents an independent examination of TGA test run data from sixteen $\mathrm{K}$ Basin fuel samples. Time-average linear oxidation rates were derived from the TGA data. The analysis did not address complex oxidation mechanisms or experimental anomalies in deriving the oxidation rate data. Statistical methods were utilized to compare these 1 imited data to the 1 arger body of literature data for unirradiated uranium collected by Pearce (1989). Rate law equations for oxidation using Arrhenius reaction rate theory were used in the analysis. 
HNF-SD-SNF-CN-035, Rev. 1

This page intentionally left blank. 


\subsection{SUMMARY RESULTS}

Linear oxidation rates were derived from the TGA test data for sixteen $K$ Basin fuel (SNF) samples. The tests were performed in dry air at temperatures ranging from $69{ }^{\circ} \mathrm{C}$ to $400{ }^{\circ} \mathrm{C}$. The weight gain data curves from nine of the tests were erratic indicating complex oxidation mechanisms and/or test equipment/configuration problems. The remaining seven tests produced relatively smooth data curves indicating a more classical behavior. A timeaveraged linear oxidation rate was computed for each of the sample runs. These rates were statistically compared to the literature data for dry air oxidation of unirradiated uranium.

Differences were found between the 1 iterature data and the $K$ Basin SNF data. Five of the sixteen data were outside the $95 \%$ prediction interval for unirradiated uranium data with four of these at temperatures below $150{ }^{\circ} \mathrm{C}$. Rate law constants from regressions of the SNF data were statistically different than those for the Titerature data. The regressions predict higher oxidation rate for SNF below about $150^{\circ} \mathrm{C}$ than for unirradiated uranium. Above about $250{ }^{\circ} \mathrm{C}$ SNF oxidation rate did not exceed unirradiated uranium oxidation. 
HNF-SD-SNF-CN-035, Rev. 1

This page intentionally left blank. 
HNF-SD-SNF-CN-035, Rev. 1

\subsection{ASSUMPTIONS}

The $K$ Basin fuel TGA data were evaluated using the following assumptions:

- The equipment used in the tests performed adequately producing accurate and representative information

- Oniy positive linear slopes of significant portions of the data provide oxidation rate information

- Complex mechanistic judgements about the data were not required to evaluate a test for oxidation rate information

- Time-weighted averages of the positive linear slopes represents an overal1 linear oxidation rate for each test.

The time-average oxidation rates derived from the test data were compared to a collection of literature data for oxidation of unirradiated uranium using the following assumptions:

- The test samples adequately represented $K$ Basin fuel material

- The K Basin fuel samples and test procedures were sufficiently similar to those of the literature data that valid comparisons could be made. 
HNF-SD-SNF-CN-035, Rev. 1

This page intentionally left blank. 
HNF-SD-SNF-CN-035, Rev. 1

\subsection{SOURCES OF INFORMATION}

The literature data used in this analysis were obtained from Pearce (1989) and are 1isted in Appendix A. The TGA oxidation measurements of the $K$ Basin fuel samples were performed by Pacific Northwest National Laboratory (PNNL) and are documented in Abrefah, et al., (1998). The TGA weight gain data from each of the sixteen test runs evaluated are presented in Appendix $C$. 
HNF-SD-SNF-CN-035, Rev. 1

This page intentionally left blank. 


\subsection{ANALYSIS OF DATA}

The TGA data plots for the $K$ Basin fuel (SNF) sample runs were examined, and a time-averaged oxidation rate was derived using the positive slopes from the data curve. The $K$ Basin fuel oxidation rate data were then statistically compared to oxidation rate data from the 1iterature for unirradiated uranium. In addition the rate 1 aw parameters (constants) were determined from 1 inear regressions of various sets of the $K$ Basin fuel data and the literature data, and these regression parameters were statistically evaluated to determine whether the rate 1 aw parameters were equivalent among the data sets.

\subsection{OXIDATION RATE DATÁ FROM THE LITERATURE}

A collection of oxidation rate data from the literature for unirradiated uranium in dry air was documented by Pearce (1989). The data represent 1 inear oxidation kinetics from multiple literature sources. These literature data, within the temperature range of interest, are tabulated in Appendix $A$ and are presented on an Arrhenius plot (logarithm of the rate versus inverse absolute temperature) on Figure 1. The rate law (regression line) for oxidation and the $95 \%$ prediction interval for the regression are shown on Figure 1 .

Figure 1. Literature Data for Linear Oxidation of Unirradiated Uranium in Dry Air at Temperatures below $400{ }^{\circ} \mathrm{C}$.

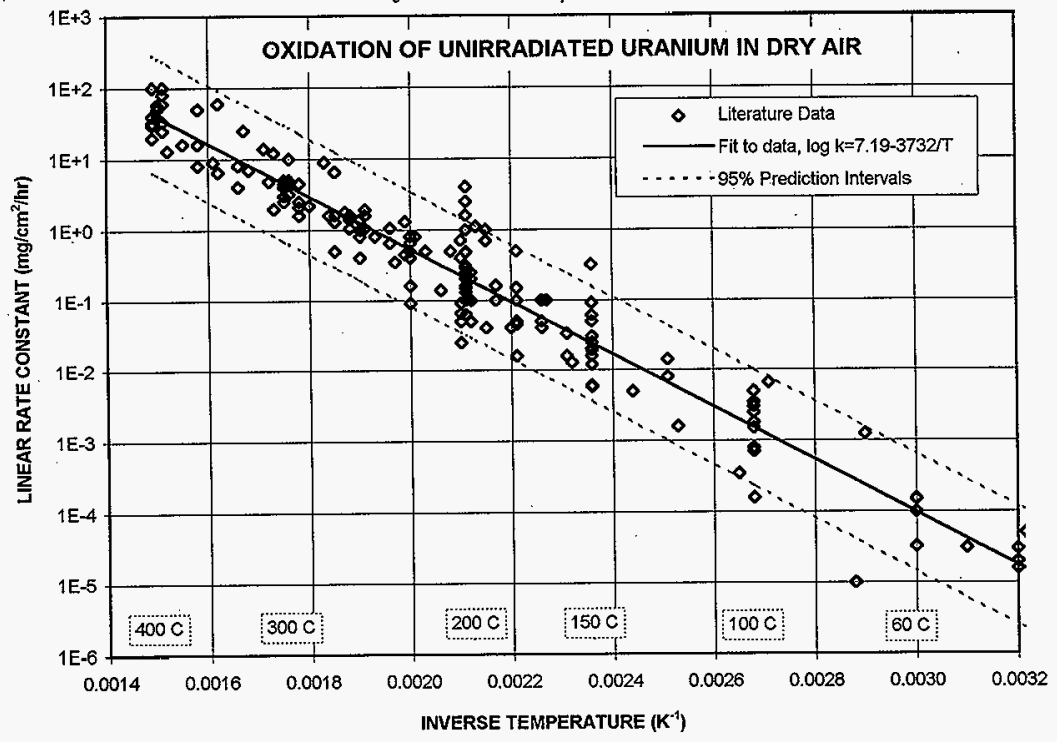




\subsection{K BASIN FUEL TEST DATA}

Plots of the TGA data for the sixteen $K$ Basin fuel samples used in this analysis are provided in Appendix $B$. These plots include all the data that were collected in the TGA runs. The analyses described in Section 5.3 used all the data in the plots except those indicated in Appendix B.

An inspection of the TGA data plots for SNF samples indicates two general categories of data. These categories are based on comparisons with similar literature data and consistency of the progressing weight change. The weight gain curves are compared in Figures 2 through 6 using weight gain units of $\left(\mathrm{mg} / \mathrm{cm}^{2}\right)$.

Normal: Seven TGA runs (19A, 20, 23, 47, 48, 49a, and 51 which are illustrated in Figure 2) are typical of the classical oxidation behavior for metallic uranium reacting in air (Ritchie 1984) and for this discussion are considered "normal." The curves are smooth with generally decreasing rates of oxidation with the rates becoming linear after long exposure times. In some cases, the reaction rate will accelerate before becoming linear (Ritchie 1984). This behavior was exhibited by Run 47. The jagged appearance of Run 48 has been attributed to equipment problems, a smoothing 7 ine through the points would give a curve appearing similar to that for Runs $19 \mathrm{a}$ and 20 . Runs $8\left(400{ }^{\circ} \mathrm{C}\right)$ and $49 \mathrm{~b}\left(124{ }^{\circ} \mathrm{C}\right)$ exhibited normal behaviors through much of the test, and then oxidation accelerated by factors of 90 to 200 . They were thus placed in the "erratic" category.

Erratic: Nine runs were categorized as "erratic". Seven runs are quite irregular and at times erratic in their behavior (Figures 3 through 6 ) and two runs ( 8 and $49 \mathrm{~b}$ in Figure 2) were somewhat erratic. Figures 3 and 4 also include for reference purposes the "normal" runs at the respective test temperatures. The "erratic" curves generally exhibited a wide range of slopes that included long periods of low weight gain ending in short periods of rapid weight gain. In some cases an initial period of weight loss was observed (Runs 7 and 18). Long periods of low weight gain were generally quite variable. In contrast the relatively rapid rates of increasing weight occurred over short time periods and were, at times, followed by rapid and significant weight loss (Runs $7,18,18 \mathrm{~b}$, and 22) or very s7ow and/or variable weight gain (Runs $6,6 r, 8,21 a$, and 49b). These characteristics are not consistent with the typical normal weight gain curve, indicating abrupt changes in the rate of weight gain (increases and losses) and possible malfunctioning of equipment.

\subsection{OXIDATION RATE CONSTANTS FROM $K$ BASIN FUEL TESTS}

A large variability was noted in the shape of many of the TGA curves from the $K$ Basin fuel tests. The changing slopes within a given test seemed to indicate oxidation rates that were both increasing and decreasing with time. The variability of the magnitude of the change was, in some cases, an order of magnitude or more. In general the high slopes persisted for short time periods relative to the lower rates. Due to this great variability, standard methods for deriving a single oxidation rate to 
Figure 2. Data Plots for $K$ Basin Fuel Oxidation Test Runs Exhibiting Normal or Near Normal Behavior.

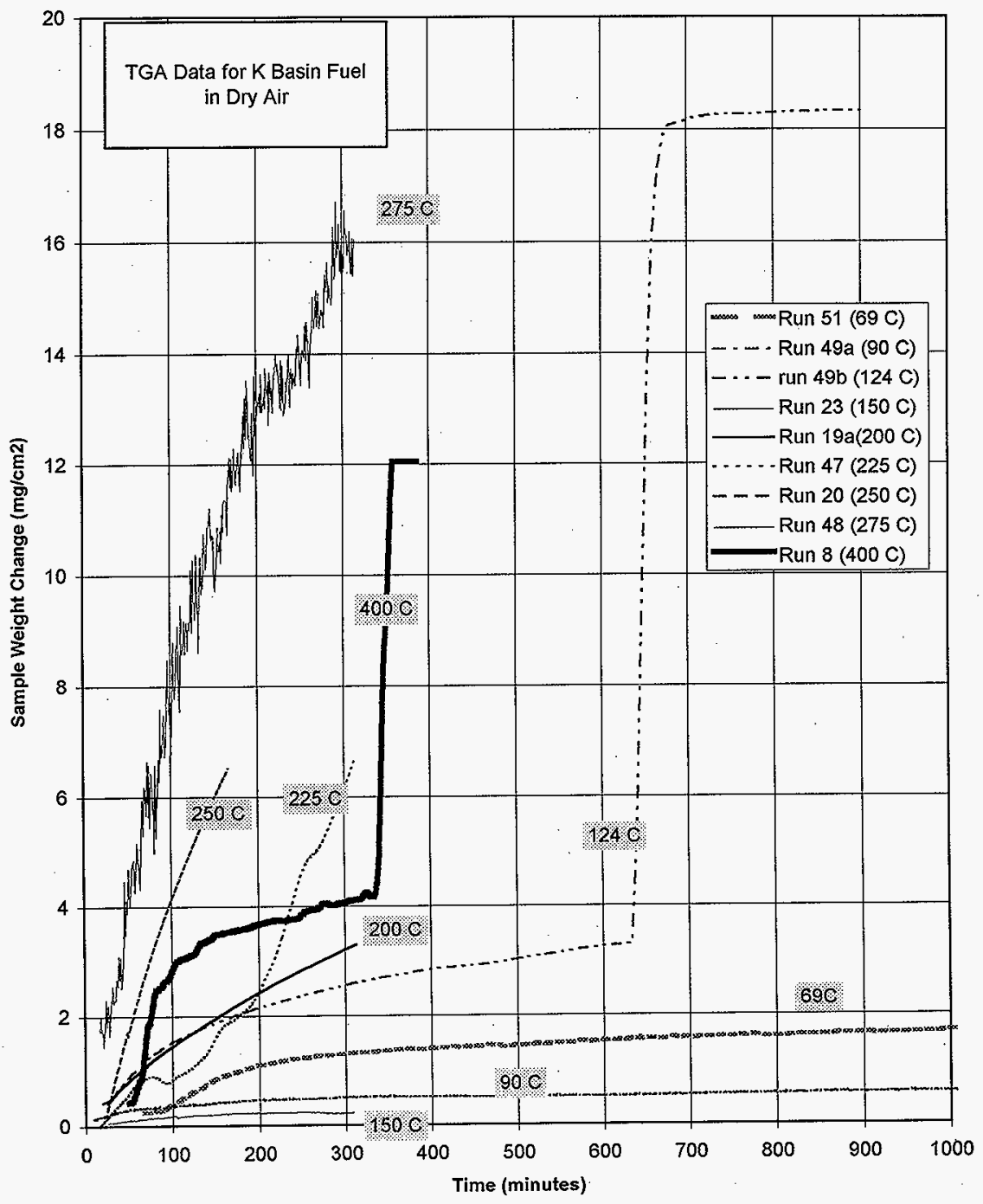


Figure 3. Thermo-Gravimetric Analysis Data Plots for $\mathrm{K}$ Bas in Fuel Sample Runs at $144^{\circ} \mathrm{C}$ and $150{ }^{\circ} \mathrm{C}$.

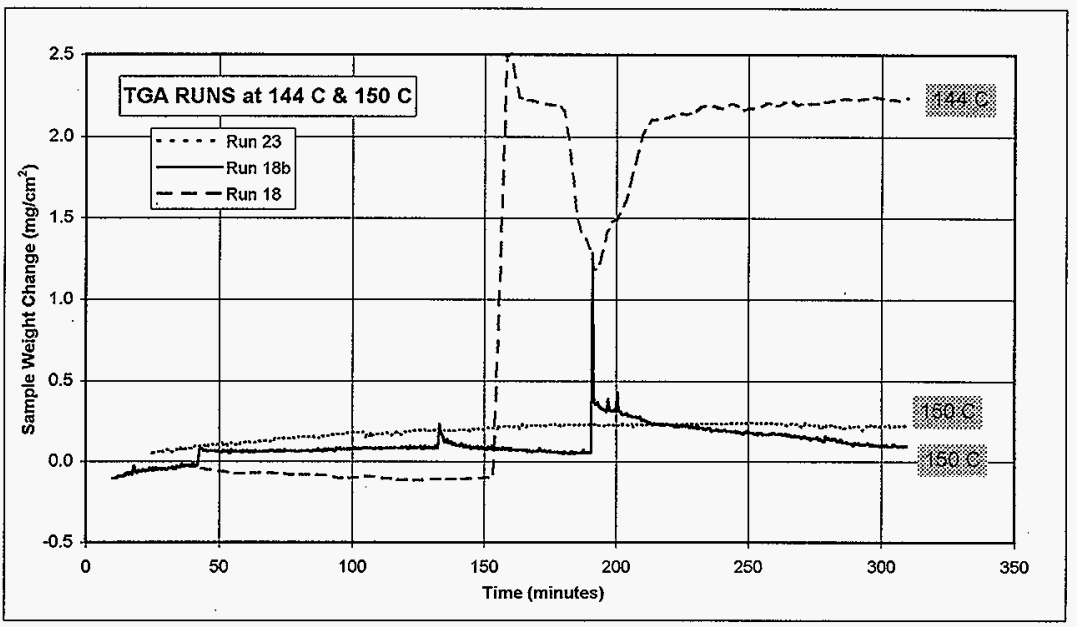

Figure 4. Thermo-Gravimetric Analysis Data Plots for $\mathrm{K}$ Basin Fuel Sample Runs at $200{ }^{\circ} \mathrm{C}$.

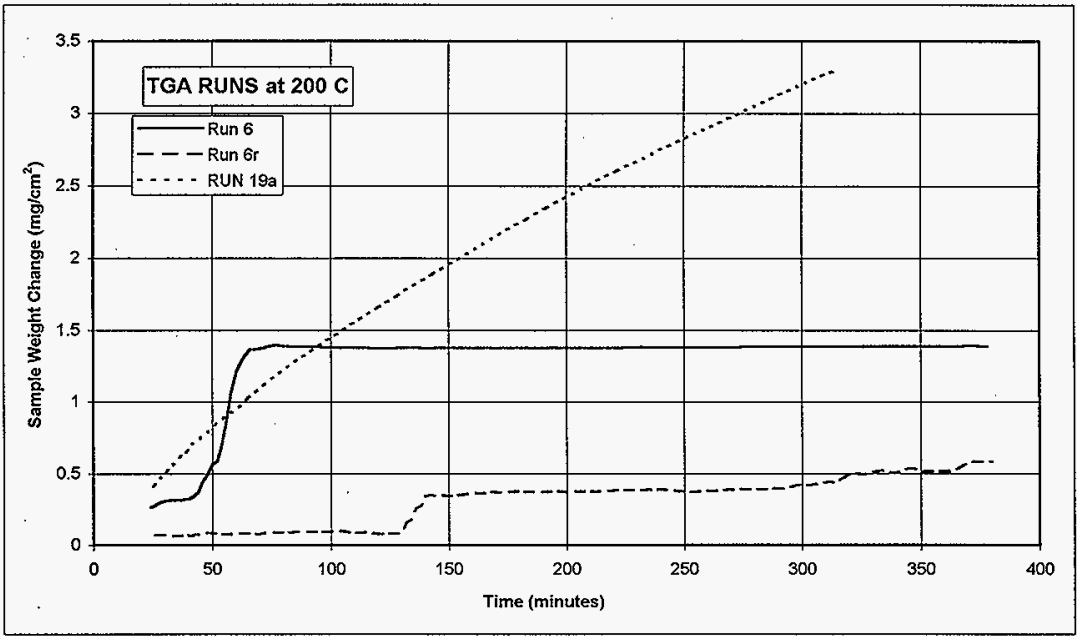


Figure 5. Thermo-Gravimetric Analysis Data Plots for $K$ Basin Fuel Sample Runs at $300{ }^{\circ} \mathrm{C}$.

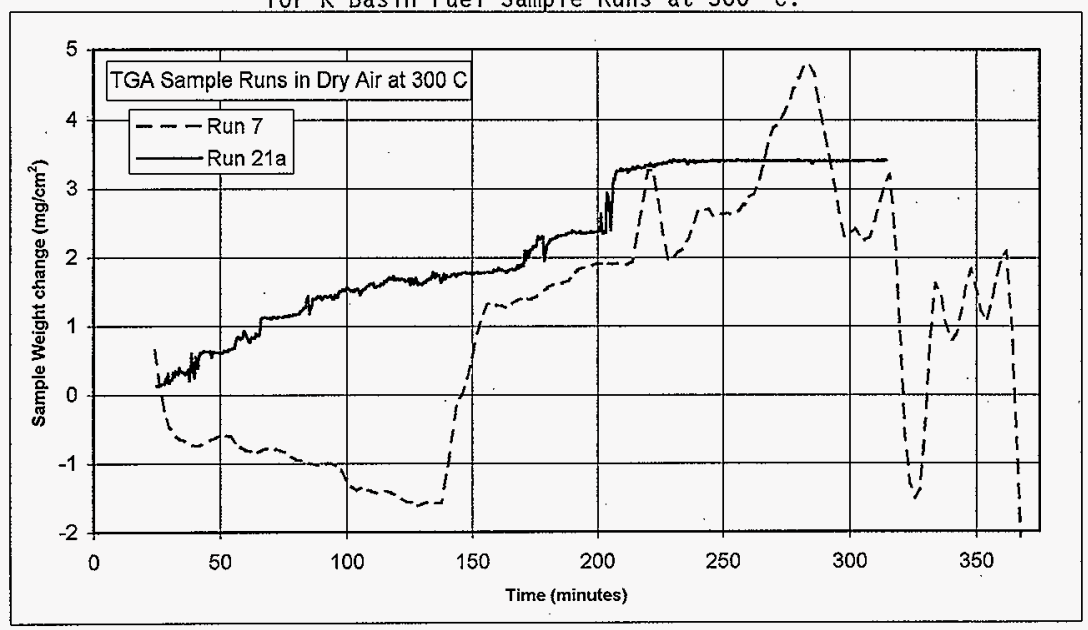

Figure 6. Thermo-Gravimetric Analysis Data Plots for $\mathrm{K}$ Basin Fuel Sample Runs at $350^{\circ} \mathrm{C}$ and $400^{\circ} \mathrm{C}$.

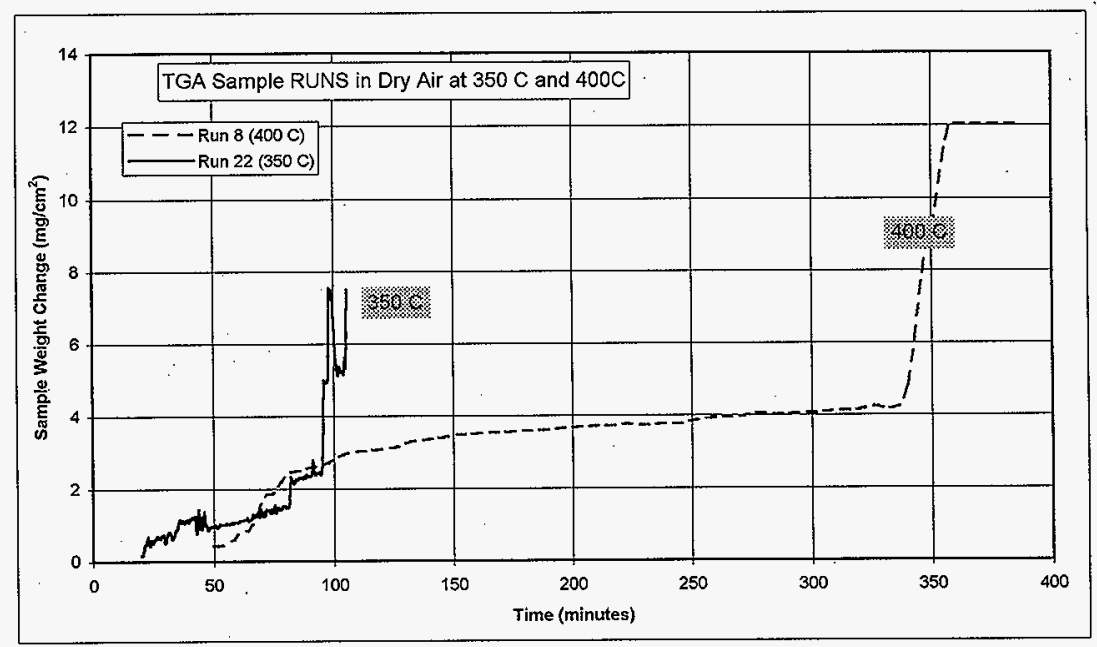


represent a given test were not possible without making mechanistic or subjective judgements about the data. Thus, a method for calculating a time-weighted average linear rate was developed. This method utilized all statistically significant positive slopes.

Slopes were derived using linear regression techniques on the linear regions of the data curves. A minimum of four observations (data points) were used for each slope. Only time intervals which showed positive weight changes were used, although occasional momentary indications of weight loss were ignored in selecting a positive slope range. All slopes selected were statistically different from zero at the 0.05 level of significance.

The analyses of the regression results from the $T$ inear regions of each TGA run are illustrated in Appendix B. In most cases the coefficient of determination $\left(R^{2}\right)$ for a regression exceeded $80 \%$. The $R^{2}$ value is an indicator of the strength of association between variables of the regression relation. It is the proportion of the variability in the dependent variable (weight change) that is explained by the independent variable (time). In a few cases, the regression analysis had an $R^{2}$ value less than $80 \%$ due to a wider data scatter in the time interval evaluated.

The oxidation rate in milligrams of weight gain per square centimeter of exposed uranium per hour $\left(\mathrm{mg} / \mathrm{cm}^{2} / \mathrm{hr}\right)$ was calculated from the slopes of each data curve. Sample surface areas had been estimated from measurements of the geometric surface areas of the as cut samples. A time-weighted average oxidation rate was then calculated for each TGA run. The data from all positive slopes were used in the average. Sample information, regression results and rate data are listed in Table 1 .

The slope corresponding to the time-weighted average oxidation rate is plotted with the data curves in Appendix B. These plots illustrate how oxidation at the time-average rate compares with the test data.

\subsection{COMPARISON OF $\mathrm{K}$ BASIN FUEL OXIDATION RATES WITH LITERATURE DATA}

Figure 7 is an Arrhenius plot of the time-averaged oxidation rate data from each of the $\mathrm{K}$ Basin fuel TGA runs. Al so shown in Figure 7 are the regression 1 ine and $95 \%$ prediction interval for the literature data shown in Figure 1. Note that five of the $K$ Basin fuel oxidation rate data points fall wel1 outside the $95 \%$ prediction interval calculated from the literature data.

The logarithm (Base 10) of the oxidation rates from the $K$ Basin fuel TGA data were combined with the logarithm (Base 10) of the oxidation rates from the literature data at similar temperatures (eleven groups of data were constructed, Table 2). Outlier tests (ASTM 1969) were performed for each group of data to determine if the $K$ Basin fuel data were consistent with the literature data. The $\mathrm{K}$ Basin fuel sample oxidation rate at $124{ }^{\circ} \mathrm{C}, 144{ }^{\circ} \mathrm{C}$, and $400^{\circ} \mathrm{C}$ tested as "outliers" while all other $\mathrm{K}$ Basin fuel data did not test as outlier data relative to the literature data. 
Table 1. Sample Data and Results from Thermo-Gravimetric Analysis Tests of K Basin Fuel. (Page 1 of 2).

\begin{tabular}{|c|c|c|c|c|c|c|c|c|}
\hline \multicolumn{2}{|c|}{ RUN } & \multirow{3}{*}{$\begin{array}{c}\text { Sample } \\
\text { Area } \\
\left(\mathrm{cm}^{2}\right) \\
\end{array}$} & \multicolumn{4}{|c|}{ SLOPE DATA } & \multirow{2}{*}{\multicolumn{2}{|c|}{$\begin{array}{c}\text { Oxidation Rate } \\
\left(\mathrm{mg} / \mathrm{cm}^{2} / \mathrm{hr}\right)\end{array}$}} \\
\hline \multirow[t]{2}{*}{ Number } & \multirow{2}{*}{$\begin{array}{l}\text { Temp. } \\
\operatorname{deg} \mathrm{C}\end{array}$} & & \multirow[t]{2}{*}{ No. } & \multirow{2}{*}{$\begin{array}{l}\text { Duration }\left(\mathrm{t}^{*}{ }^{*}\right. \\
\text { (min.) }\end{array}$} & \multirow{2}{*}{$\begin{array}{c}\begin{array}{c}\text { Value } \\
\text { (mg/min) }\end{array} \\
\end{array}$} & \multirow[t]{2}{*}{$R^{2}$} & & \\
\hline & & & & & & & Slope ${ }^{* \hbar t}$ & Áverage ${ }^{* *}$ \\
\hline \multirow{4}{*}{6} & \multirow{4}{*}{200} & \multirow{4}{*}{3.02} & 1 & 20.0 & $1.19 \mathrm{E}-2$ & 0.887 & $2.4 \mathrm{E}-1$ & \multirow{4}{*}{0.22472} \\
\hline & & & 2 & 18.0 & $1.67 \mathrm{E}-1$ & 0.963 & $3.3 \mathrm{E}+0$ & \\
\hline & & & 3 & 10.0 & $9.50 \mathrm{E}-3$ & 0.978 & $1.9 \mathrm{E}-1$ & \\
\hline & & & 4 & 252.0 & $1.94 \mathrm{E}-4$ & 0.962 & $3.9 \mathrm{E}-3$ & \\
\hline \multirow{7}{*}{$6 r$} & \multirow{7}{*}{200} & \multirow{7}{*}{2.96} & 1 & 72.0 & $1.27 \mathrm{E}-3$ & 0.852 & $2.6 \mathrm{E}-2$ & \multirow{7}{*}{0.139} \\
\hline & & & 2 & 14.0 & $6.34 \mathrm{E}-2$ & 0.970 & $1.3 \mathrm{E}+0$ & \\
\hline & & & 3 & 16.0 & $3.34 \mathrm{E}-3$ & 0.901 & $6.8 \mathrm{E}-2$ & \\
\hline & & & 4 & 68.0 & $7.35 E-4$ & 0.884 & $1.5 \mathrm{E}-2$ & \\
\hline & & & 5 & 24.0 & $2.54 \mathrm{E}-3$ & 0.978 & $5.1 \mathrm{E}-2$ & \\
\hline & & & 6 & 66.0 & $7.43 \mathrm{E}-3$ & 0.952 & $1.5 \mathrm{E}-1$ & \\
\hline & & & 7 & 12.0 & $1.89 \mathrm{E}-2$ & 0.954 & $3.8 \mathrm{E}-1$ & \\
\hline \multirow{9}{*}{7} & & & 1 & 18.0 & $4.44 E-1$ & 0.975 & $9.3 E+0$ & \\
\hline & & & 2 & 54.0 & $4.06 \mathrm{E}-2$ & 0.939 & $8.5 \mathrm{E}-1$ & \\
\hline & & & 3 & 6.0 & $6.35 \mathrm{E}-1$ & 0.998 & $1.3 \mathrm{E}+1$ & 5.68 \\
\hline & & & 4 & 12.0 & $1.65 \mathrm{E}-1$ & 0.902 & $3.4 \mathrm{E}+0$ & \\
\hline & 300 & 2.87 & 5 & 30.0 & $2.31 \mathrm{E}-1$ & 0.983 & $4.8 \mathrm{E}+0$ & \\
\hline & & & 6 & 10.0 & $2.99 \mathrm{E}-1$ & 0.967 & $6.3 \mathrm{E}+0$ & \\
\hline & & & 7 & 8.0 & $1.21 E+0$ & 0.936 & $2.5 \mathrm{E}+1$ & \\
\hline & & & 8 & 8.0 & $4.03 E-1$ & 0.953 & $8.4 \mathrm{E}+0$ & \\
\hline & & & 9 & 8.0 & $3.83 \mathrm{E}-1$ & 0.959 & $8.0 \mathrm{E}+0$ & \\
\hline & & & 1 & 12.0 & $6.55 \mathrm{E}-2$ & 0.926 & $2.0 \mathrm{E}+0$ & \\
\hline & & & 2 & 16.0 & $1.84 \mathrm{E}-1$ & 0.970 & $5.7 \mathrm{E}+0$ & \\
\hline 8 & 400 & 1.95 & 3. & 115.0 & $2.90 \mathrm{E}-2$ & 0.967 & $8.9 \mathrm{E}-1$ & 1.93 \\
\hline & & & 4 & 242.0 & $8.10 \mathrm{E}-3$ & 0.983 & $2.5 \mathrm{E}-1$ & \\
\hline & & & 5 & 22.0 & $7.51 \mathrm{E}-1$ & 0.989 & $2.3 \mathrm{E}+1$ & \\
\hline & & & 1 & 6.7 & $1.07 \mathrm{E}+0$ & 0.946 & $2.5 \mathrm{E}+1$ & \\
\hline 18 & 144 & 2.54 & 2 & 18.3 & $1.17 \mathrm{E}-1$ & 0.977 & $2.8 \mathrm{E}+0$ & \\
\hline & & & 3 & 21.7 & $1.11 \mathrm{E}-2$ & 0.933 & $2.6 \mathrm{E}-1$ & 1.93 \\
\hline & & & 4 & 71.7 & $2.34 \mathrm{E}-3$ & 0.794 & $5.5 \mathrm{E}-2$ & \\
\hline & & & 1 & 7.5 & 1.17E-2 & 0.813 & $2.7 \mathrm{E}-1$ & \\
\hline $18 \mathrm{~b}$ & 150 & 2.62 & 2 & 22.0 & $5.10 E-3$ & 0.800 & $1.2 \mathrm{E}-1$ & \\
\hline & & & 3 & 2.5 & $1.42 \mathrm{E}-1$ & 0.813 & $3.3 E+0$ & 0.127 \\
\hline & & & 4 & 83.5 & $1.00 \mathrm{E}-3$ & 0.789 & $2.3 \mathrm{E}-2$ & \\
\hline & & & 1 & 30.0 & $4.08 \mathrm{E}-2$ & 0.997 & $9.6 \mathrm{E}-1$ & \\
\hline & & & 2 & 35.0 & $3.26 \mathrm{E}-2$ & 0.999 & $7.6 \mathrm{E}-1$ & \\
\hline & & & 3 & 30.0 & $2.70 \mathrm{E}-2$ & 1.000 & $6.3 \mathrm{E}-1$ & 0.600 \\
\hline $19 a$ & 200 & 2.56 & 4 & 40.0 & $2.51 \mathrm{E}-2$ & 1.000 & $5.9 \mathrm{E}-1$ & \\
\hline & & & 5 & 60.0 & $2.24 \mathrm{E}-2$ & 1.000 & $5.3 \mathrm{E}-1$ & \\
\hline & & & 6 & 85.0 & $1.93 \mathrm{E}-2$ & 1.000 & $4,5 \mathrm{E}-1$ & \\
\hline & & & 1 & 23.4 & $1.53 \mathrm{E}-1$ & 1.000 & $3.7 E+0$ & \\
\hline & & & 2 & 11.5 & $1.41 \mathrm{E}-1$ & 1.000 & $3.4 \mathrm{E}+0$ & \\
\hline 20. & 250 & 2.50 & 3 & 6.2 & $1.34 \mathrm{E}-1$ & 0.999 & $3.2 \mathrm{E}+0$ & 2.69 \\
\hline & & & 4 & 7.7 & $1.24 \mathrm{E}-1$ & 1.000 & $3.0 \mathrm{E}+0$ & \\
\hline & & & 5 & 11.5 & $1.15 \mathrm{E}-1$ & 1.000 & $2.8 \mathrm{E}+0$ & \\
\hline & & & 6 & 19.6 & $1.04 \mathrm{E}-1$ & 1.000 & $2.5 \mathrm{E}+0$ & \\
\hline & & & 7 & 14.8 & $9.57 \mathrm{E}-2$ & 1.000 & $2.3 \mathrm{E}+0$ & \\
\hline & & & 8 & 9.6 & $9.12 \mathrm{E}-2$ & 1.000 & $2.2 \mathrm{E}+0$ & \\
\hline & & & 9 & 19.1 & $8.56 \mathrm{E}-2$ & 1.000 & $2.1 \mathrm{E}+0$ & \\
\hline & & & 10 & 13.4 & $8.06 \mathrm{E}-2$ & 1.000 & $1.9 \mathrm{E}+0$ & \\
\hline
\end{tabular}


Table 1. Sample Data and Results from Thermo-Gravimetric Analysis Tests of $\mathrm{K}$ Basin Fuel. (Page 2 of 2)

\begin{tabular}{|c|c|c|c|c|c|c|c|c|}
\hline \multicolumn{2}{|c|}{ RUN } & \multirow{3}{*}{$\begin{array}{c}\text { Sample } \\
\text { Area } \\
\left(\mathrm{cm}^{2}\right)\end{array}$} & \multicolumn{4}{|c|}{ SLOPE DATA } & \multirow{2}{*}{\multicolumn{2}{|c|}{$\begin{array}{l}\text { Oxidation Rate } \\
\left(\mathrm{mg} / \mathrm{cm}^{2} / \mathrm{hr}\right)\end{array}$}} \\
\hline \multirow[t]{2}{*}{ Number } & \multirow{2}{*}{$\begin{array}{l}\text { Temp. } \\
\text { deg C }\end{array}$} & & \multirow[t]{2}{*}{ No. } & \multirow{2}{*}{$\begin{array}{l}\text { Duration }\left(\mathrm{t}^{*}\right. \\
\text { (min.) }\end{array}$} & \multirow{2}{*}{$\begin{array}{c}\text { Value } \\
(\mathrm{mg} / \mathrm{min})\end{array}$} & \multirow[t]{2}{*}{$\mathrm{R}^{2}$} & & \\
\hline & & & & & & & Slope & Average $^{\star k}$ \\
\hline \multirow{7}{*}{$21 a$} & \multirow{7}{*}{300} & \multirow{7}{*}{2.51} & 1 & 61.3 & $4.92 \mathrm{E}-2$ & 0.960 & $1.2 \mathrm{E}+0$ & \multirow{7}{*}{1.04} \\
\hline & & & 2 & 33.8 & $2.29 \mathrm{E}-2$ & 0.896 & $5.5 \mathrm{E}-1$ & \\
\hline & & & 3 & 46.6 & $1.04 \mathrm{E}-2$ & 0.794 & $2.5 E-1$ & \\
\hline & & & 4 & 10.3 & 1.22E-1 & 0.919 & $2.9 \mathrm{E}+0$ & \\
\hline & & & 5 & 10.3 & $4.01 \mathrm{E}-2$ & 0.915 & $9.6 \mathrm{E}-1$ & \\
\hline & & & 6 & 1.5 & $1.32 \mathrm{E}+0$ & 0.815 & $3.2 E+1$ & \\
\hline & & & 7 & 27.4 & $1.55 \mathrm{E}-2$ & 0.930 & $3.7 \mathrm{E}-1$ & \\
\hline \multirow{5}{*}{22} & \multirow{5}{*}{350} & \multirow{5}{*}{2.51} & 1 & 22.4 & $1.02 \mathrm{E}-1$ & 0.874 & $2.4 \mathrm{E}+0$ & \multirow{5}{*}{7.72} \\
\hline & & & 2 & 34.0 & $4.87 \mathrm{E}-2$ & 0.936 & $1.2 E+0$ & \\
\hline & & & 3 & 8.8 & $4.30 \mathrm{E}-2$ & 0.516 & $1.0 \mathrm{E}+0$ & \\
\hline & & & 4 & 4.7 & $2.91 \mathrm{E}+0$ & 0.887 & $7.0 E+1$ & \\
\hline & & & 5 & 1.4 & $3.67 \mathrm{E}+0$ & 0.649 & $8.8 \mathrm{E}+1$ & \\
\hline \multirow{5}{*}{23} & \multirow{5}{*}{150} & \multirow{5}{*}{1.24} & 1 & 79.5 & $1.96 \mathrm{E}-3$ & 0.979 & 9.5E-2 & \multirow{5}{*}{0.0549} \\
\hline & & & 2 & 21.5 & $2.02 \mathrm{E}-3$ & 0.849 & $9.8 \mathrm{E}-2$ & \\
\hline & & & 3 & 46.5 & $1.14 \mathrm{E}-3$ & 0.897 & $5.5 \mathrm{E}-2$ & \\
\hline & & & 4 & 76.0 & $1.94 \mathrm{E}-4$ & 0.605 & $9.4 \mathrm{E}-3$ & \\
\hline & & & 5 & 25.5 & $6.14 \mathrm{E}-4$ & 0.442 & $3.0 \mathrm{E}-2$ & \\
\hline \multirow{8}{*}{47} & \multirow{8}{*}{225} & & 1 & 63.0 & $3.72 \mathrm{E}-2$ & 0.989 & 9.7E-1 & \\
\hline & & & 2 & 45.0 & $2.57 \mathrm{E}-2$ & 0.988 & $6.7 \mathrm{E}-1$ & \\
\hline & & 2.29 & 3. & 14.0 & $6.62 \mathrm{E}-2$ & 0.999 & $1.7 \mathrm{E}+0$ & 1.45 \\
\hline & & & 4 & 38.0 & $2.79 \mathrm{E}-2$ & 0.986 & $7.3 \mathrm{E}-1$ & \\
\hline & & & 5 & 22.0 & $7.20 \mathrm{E}-2$ & 1.000 & $1.9 \mathrm{E}+0$ & \\
\hline & & & 6 & 33.0 & $1.15 \mathrm{E}-1$ & 1.000 & $3.0 \mathrm{E}+0$ & \\
\hline & & & 7 & 21.0 & $4.40 \mathrm{E}-2$ & 0.982 & $1.2 \mathrm{E}+0$ & \\
\hline & & - & 8 & 38.0 & $8.99 \mathrm{E}-2$ & 0.999 & $2.4 \mathrm{E}+0$ & \\
\hline & & & 1 & 133.0 & $1.65 \mathrm{E}-1$ & 0.972 & $3.8 \mathrm{E}+0$ & \\
\hline 48 & 275 & 2.62 & 2 & 26.0 & $1.89 \mathrm{E}-1$ & 0.806 & $4.3 \mathrm{E}+0$ & 2.76 \\
\hline & & & 3 & 136.0 & $6.43 \mathrm{E}-2$ & 0.874 & $1.5 \mathrm{E}+0$ & \\
\hline & & & 1 & 42.0 & $1.02 \mathrm{E}-2$ & 0.985 & $2.6 \mathrm{E}-1$ & \\
\hline $49 a$ & 90 & 2.41 & 2 & 159.0 & $2.59 \mathrm{E}-3$ & 0.986 & $6.5 \mathrm{E}-2$ & 0.0231 \\
\hline & & & 3 & 997.0 & $2.70 \mathrm{E}-4$ & 0.928 & $6.7 \mathrm{E}-3$ & \\
\hline & & & 1 & 100.0 & $3.11 \mathrm{E}-2$ & 0.982 & $7.8 \mathrm{E}-1$ & \\
\hline & & & 2 & $186.7^{\circ}$ & $1.12 \mathrm{E}-2$ & 0.990 & $2.8 E-1$ & \\
\hline $49 b$ & 124 & 2.39 & 3 & 326.7 & $5.22 \mathrm{E}-3$ & 0.995 & 1.3E-1 & 1.25 \\
\hline & & & 4 & 33.3 & $1.09 \mathrm{E}+0$ & 0.980 & $2.7 \mathrm{E}+1$ & \\
\hline & & & 5 & 233.3 & $2.40 \mathrm{E}-3$ & 0.636 & $6.0 \mathrm{E}-2$ & \\
\hline & & & 1 & 109.7 & $1.84 \mathrm{E}-2$ & 0.983 & $4.6 \mathrm{E}-1$ & \\
\hline 51 & 69 & 2.41 & 2 & 92.0 & $3.50 \mathrm{E}-3$ & 0.960 & $8.7 E-2$ & 0.039 \\
\hline & & & 3 & 4347.0 & $1.10 \mathrm{E}-3$ & 0.992 & 2.7E-2 & \\
\hline
\end{tabular}

* Slope time is duration of measurement.

** Slope rate $=$ slope $(\mathrm{mg} / \mathrm{min}){ }^{*} 60(\mathrm{~min} / \mathrm{hr}) /$ sample area $\left(\mathrm{cm}^{\wedge} 2\right)$

Average rate $=\{$ sum of [slope rate $*$ time] $/$ sum of [times] $\}$ for all slopes in a run 
Figure 7. Oxidation Rates from $\mathrm{K}$ Bas in Fue Tests and

Prediction Lines from Regression of Literature Data.

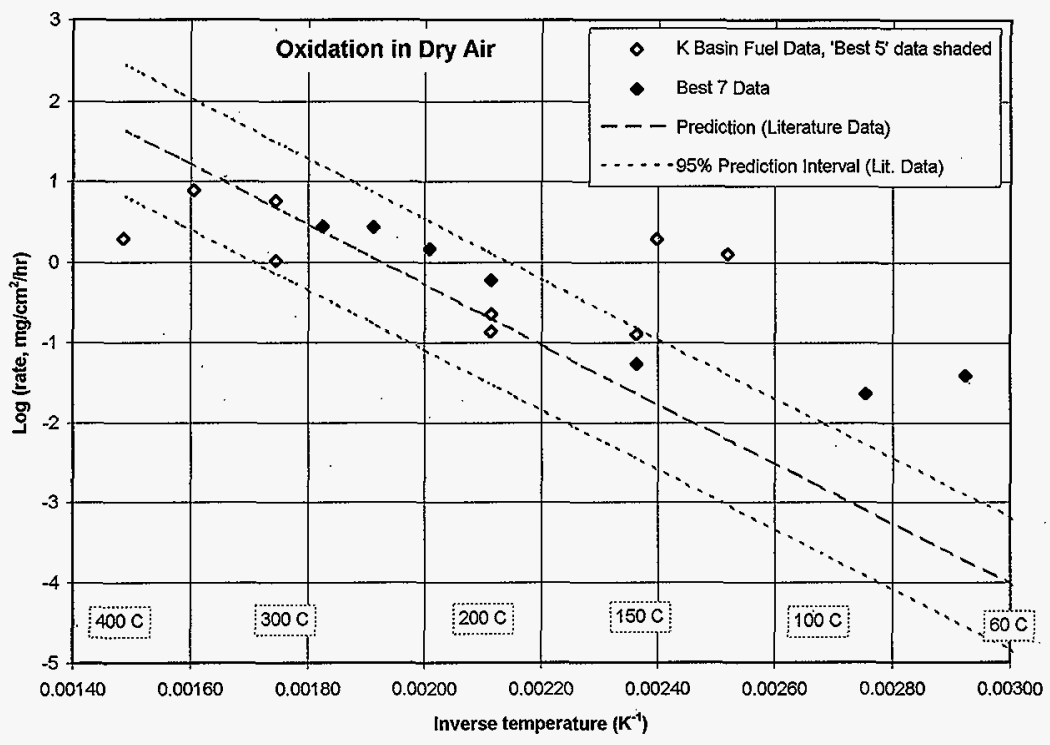

Tab7e 2. Data Groupings Used in Outlier Tests.

\begin{tabular}{|c|c|c|}
\hline & $K$ Basin Fuel Weighted Average & Literature Data \\
\hline Group 1 & $90^{\circ} \mathrm{C}, \quad 69^{\circ} \mathrm{C}(\mathrm{n}=2)$ & $44^{\circ} \mathrm{C}-94^{\circ} \mathrm{C} \quad(\mathrm{n}=6)$ \\
\hline Group 2 & $90{ }^{\circ} \mathrm{C}, \quad 69{ }^{\circ} \mathrm{C} \quad(\mathrm{n}=2)$ & $65^{\circ} \mathrm{C}-115^{\circ} \mathrm{C} \quad(n=13)$ \\
\hline Group 3 & $144{ }^{\circ} \mathrm{C}, 124{ }^{\circ} \mathrm{C} \quad(\mathrm{n}=2)$ & $99^{\circ} \mathrm{C}-149^{\circ} \mathrm{C} \quad(n=14)$ \\
\hline Group 4 & $124{ }^{\circ} \mathrm{C}, 144^{\circ} \mathrm{C}, 150^{\circ} \mathrm{C} \quad(\mathrm{n}=3)$ & $119^{\circ} \mathrm{C}-169^{\circ} \mathrm{C} \quad(\mathrm{n}=20)$ \\
\hline Group 5 & $144{ }^{\circ} \mathrm{C}, 150^{\circ} \mathrm{C} \quad(\mathrm{n}=3)$ & $125^{\circ} \mathrm{C}-175^{\circ} \mathrm{C} \quad(\mathrm{n}=22)$ \\
\hline Group 6 & $200{ }^{\circ} \mathrm{C} \quad(n=3)$ & $175^{\circ} \mathrm{C}-225^{\circ} \mathrm{C} \quad(n=44)$ \\
\hline Group 7 & $225^{\circ} \mathrm{C} \quad(n=1)$ & $200^{\circ} \mathrm{C}-250^{\circ} \mathrm{C} \quad(n=42)$ \\
\hline Group 8 & $250^{\circ} \mathrm{C} \quad(\mathrm{n}=1)$ & $225^{\circ} \mathrm{C}-275^{\circ} \mathrm{C} \quad(\mathrm{n}=29)$ \\
\hline Group 9 & $275^{\circ} \mathrm{C} \quad(n=1)$ & $250^{\circ} \mathrm{C}-300^{\circ} \mathrm{C} \quad(n=31)$ \\
\hline Group 10 & $300^{\circ} \mathrm{C} \quad(n=2)$ & $275^{\circ} \mathrm{C}-325^{\circ} \mathrm{C} \quad(n=21)$ \\
\hline Group 11 & $350^{\circ} \mathrm{C} \quad(n=1)$ & $325^{\circ} \mathrm{C}-375^{\circ} \mathrm{C} \quad(n=10)$ \\
\hline Group 12 & $400{ }^{\circ} \mathrm{C} \quad(n=1)$ & $375^{\circ} \mathrm{C}-425^{\circ} \mathrm{C} \quad(n=14)$ \\
\hline
\end{tabular}




\subsection{REGRESSION ANALYSIS OF $\mathrm{K}$ BASIN FUEL DATA}

The sixteen $K$ Basin SNF data were regressed on an Arrhenius plot. Standardized residuals from the regression are shown on Figure 8 . A standardized residual is the difference between the predicted value (LOG of oxidation rate) and the observed value divided by the standard error (MSE) of the regression analysis. The standardized residuals should be randomiy scattered about zero. Outliers are extreme observations. In a residual plot, they are points that lie far beyond the scatter of the remaining residuals (perhaps four or more standard deviations from zero). There are no standardized residuals in Figure 8 that are greater than four standard deviations from zero. However, there are two consecutive standardized residuals (Run 18 at $144{ }^{\circ} \mathrm{C}$ and Run 49 a at $124{ }^{\circ} \mathrm{C}$ ) that are approximately two standard deviations (both positive) from zero. In statistical process control (SPC) both one observation greater than three times the standard deviation from the mean and two consecutive observations greater than two times the standard deviation from the mean indicate an "out of control" situation. Thus, it is probable that the two observations which generated these two standardized residual are outliers.

Figure 8. Standardized Residuals from Regression of $K$ Basin Fuel Oxidation Rate Data.

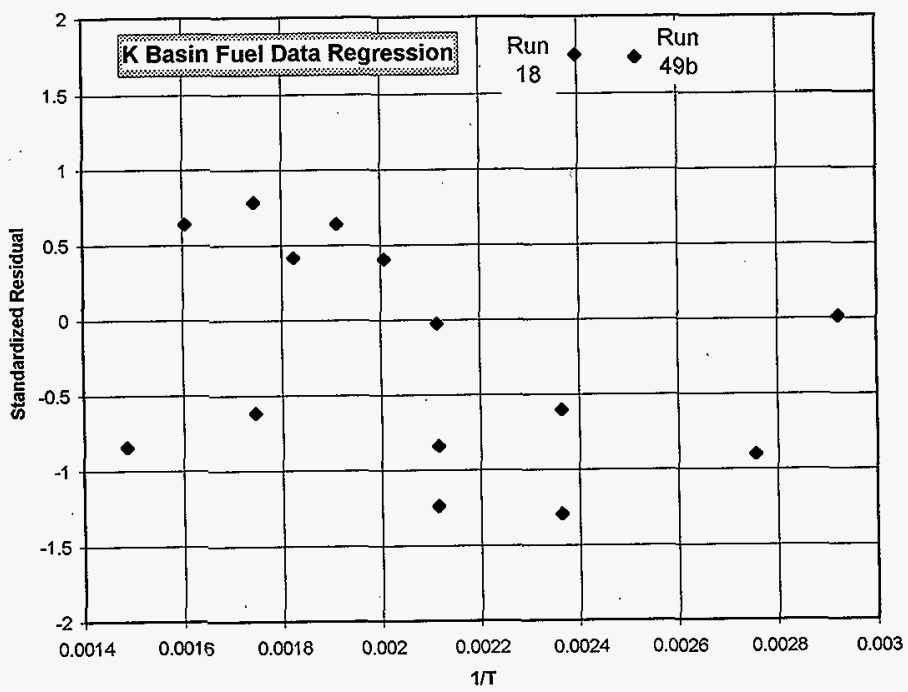


A major reason for discarding an outlier is that under the least squares method (regression analysis), a fitted line is pulled disproportionately toward an outlying observation because the sum of the squared deviations is minimized. The regression analysis using the sixteen $K$ Basin SNF data was performed using a statistical program called STATGRAPHICS.* one of the options in the regression analys is from STATGRAPHICS provides the observations which have high leverage (the data influence the regression results). The influence measure option indicated that there were two observations (Run 18 at $144{ }^{\circ} \mathrm{C}$ and Run $49 \mathrm{a}$ at $124{ }^{\circ} \mathrm{C}$ ) with high leverage. Note that these are the same two observations mentioned in the previous paragraph.

The $K$ Basin fuel data were grouped into three sets for further comparative analysis: (1) "all 16 data", (2) the "edited 14 data" (Runs 18 and $49 \mathrm{a}$ deleted), and (3) the "best 7 data" (data from "normal" runs, see Section 5.2). The regression results from the three data sets are shown on Figure 9 with the SNF data and on Figure 10 with the literature data regression lines.

Hypothesis testing was used to answer the question "Can the regression lines be regarded as the same?" The linear regressions, $1 / \mathrm{T}\left(\mathrm{K}^{-1}\right)$ as the independent variable and the logarithm of the oxjdation rate as the dependent variable, may differ in the slope, in the intercept, and in the residual variances. The usual approach is to compare (perform a hypothesis test of equality) the residuals variables first, then the slopes, and then the constants. The statistical tests (Snedecor 1980) regarding equality with respect to residual variances, slopes, and intercepts were performed for a11 pair combinations of the data sets (1 iterature versus a 11 16, literature versus "edited 14", 1 iterature versus "best 7", al1 16 versus "edited 14", al1 16 versus "best 7", and "edited 14" versus "best $7^{\prime \prime}$ ). The results (p-values) of these statistical tests are tabulated in Table 3 . The p-value (significance level of the test) indicates whether the hypothes is of equality is rejected or not rejected.

Hypothesis testing was also performed to determine if the $\mathrm{K}$ Basin fue 1 data. (weighted average for each fuel sample), when added to the literature data, made significant changes to the regression analysis of the literature data. Statistical tests (Snedecor 1980) regarding equality with respect to residual variances, slopes, and intercepts were performed for all pair combinations of the data sets (1iterature versus literature +16 , literature versus 1 iterature +14 , and Titerature versus 1 iterature +7 ). The observed significance levels ( $p$-values) of the tests are tabulated in Table 3.

A11 statistical tests were evaluated at the 0.05 significance level. That is, reject the hypothesis of equality (e.g., slope $_{1}=$ slope $_{2}$, which also can be stated as slope ${ }_{1}-$ slope $_{2}=0$ ) if the p-value is less than 0.05 . The 0.05 level of significance means that there is a $5 \%$ chance that the hypothesis of equality will be rejected when the hypothesis of equality is really true. A Jevel of $1 \%$ is sometimes used when an incorrect rejection of the hypothesis is regarded as a serious mistake. Reporting the p-value shows the actual significance of the statistical test.

*STATGRAPHICS is a trademark of Statistical Graphics Corporation, Rockville, Maryland 20852. 
Figure 9. Regression Lines for Dry Air Oxidation Rate Data from $K$ Basin Fuel Tests.

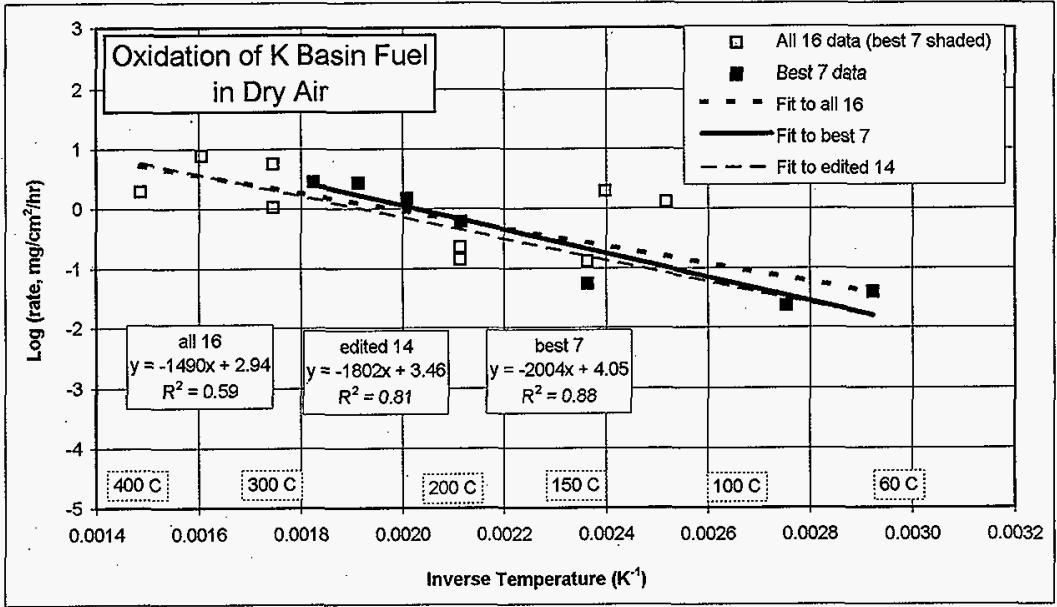

Figure 10. Comparison of Regressions of Oxidation Rate Data for Unirradiated Uranium and K Basin Fuel.

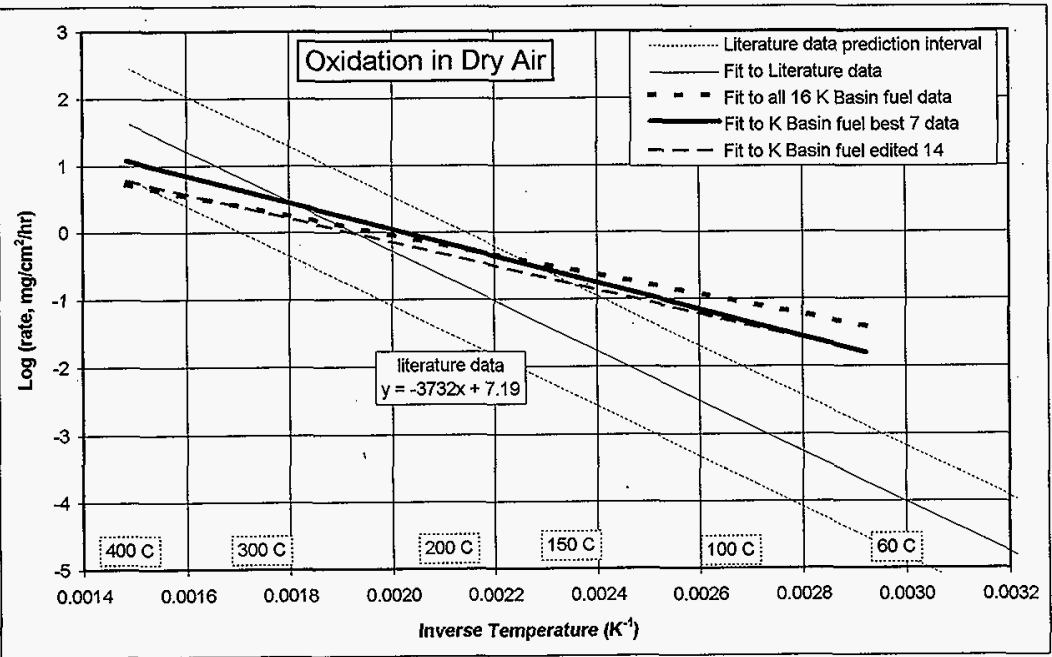


Table 3. P-Values for Comparisons of Linear Regression Constants for Various Sets of 0xidation Rate Data.

\begin{tabular}{|c|c|c|c|c|c|}
\hline Data Set & $\begin{array}{c}\text { Statistical } \\
\text { Tests }\end{array}$ & $\begin{array}{c}\text { Literature } \\
\text { Data }\end{array}$ & $\begin{array}{c}\text { K Basin } \\
\text { Fuel } \\
\text { A11 } 16\end{array}$ & $\begin{array}{l}\text { K Basin } \\
\text { Fuel } \\
\text { Edited } 14\end{array}$ & $\begin{array}{c}\text { K Basin } \\
\text { Fuel } \\
\text { Best } 7\end{array}$ \\
\hline $\begin{array}{l}\text { Literature } \\
\text { Data }\end{array}$ & $\begin{array}{l}\text { Variance } \\
\text { Slope } \\
\text { Intercept }\end{array}$ & & $\begin{array}{l}0.084^{* *} \\
0.000^{*} \\
N / A\end{array}$ & $\begin{array}{l}0.394 \\
0.000 * \\
N / A\end{array}$ & $\begin{array}{l}0.359 \\
0.000 * \\
N / A\end{array}$ \\
\hline $\begin{array}{l}\text { K Basin } \\
\text { Fue] } \\
\text { Al] } 16 \\
\end{array}$ & $\begin{array}{l}\text { Variance } \\
\text { Slope } \\
\text { Intercept }\end{array}$ & $\begin{array}{l}0.084^{* *} \\
0.000^{*} \\
N / A^{2}\end{array}$ & & $\begin{array}{l}0.136 \\
0.473 \\
0.423 \\
\end{array}$ & $\begin{array}{l}0.175 \\
0.368 \\
0.880 \\
\end{array}$ \\
\hline $\begin{array}{l}\text { K Basin } \\
\text { Fuel } \\
\text { Edited } 14 \\
\end{array}$ & $\begin{array}{l}\text { Variance } \\
\text { Slope } \\
\text { Intercept }\end{array}$ & $\begin{array}{l}0.394 \\
0.000^{*} \\
N / A\end{array}$ & $\begin{array}{l}0.136 \\
0.473 \\
0.423 \\
\end{array}$ & & $\begin{array}{l}0.432 \\
0.648 \\
0.404\end{array}$ \\
\hline $\begin{array}{l}\text { K Basin } \\
\text { Fuel } \\
\text { Best } 7\end{array}$ & $\begin{array}{l}\text { Variance } \\
\text { Slope } \\
\text { Intercept }\end{array}$ & $\begin{array}{l}0.359 \\
0.000 * \\
N / A\end{array}$ & $\begin{array}{l}0.175 \\
0.368 \\
0.880\end{array}$ & $\begin{array}{l}0.432 \\
0.648 \\
0.404 \\
\end{array}$ & \\
\hline Data Set & $\begin{array}{l}\text { Statistical } \\
\text { Tests }\end{array}$ & $\begin{array}{l}\text { Literature } \\
+ \text { A11 } 16 \\
\end{array}$ & $\begin{array}{l}\text { Literature } \\
+ \text { Edited } 14 \\
\end{array}$ & $\begin{array}{l}\text { Literature + } \\
\text { Best } 7\end{array}$ & \\
\hline $\begin{array}{l}\text { Literature } \\
\text { Data }\end{array}$ & $\begin{array}{l}\text { Variance } \\
\text { Slope } \\
\text { Intercept }\end{array}$ & $\begin{array}{l}0.003^{*} \\
0.109 \\
0.370 \\
\end{array}$ & $\begin{array}{l}0.065 * * \\
0.212 \\
0.648 \\
\end{array}$ & $\begin{array}{l}0.098 \\
0.387 \\
0.504 \\
\end{array}$ & \\
\hline
\end{tabular}

${ }^{1}$ Hypothesis of equality rejected at the 0.05 level of significance (denoted by * in the table). If the $p$-value is only marginally greater than 0.05 (denoted by **), the non-rejection of the hypothes is is suspect.

${ }^{2} \mathrm{~N} / \mathrm{A}$ : Not applicable. Testing for equality of intercepts has no meaning when the slopes are clearly not equal.

The hypothesis that the variances are equal was not rejected $(0.05$ level of significance) for all tests except the test comparing literature and 1iterature+16. Caution should be used when evaluating the two tests (1iterature compared to K Basin "a11 16" and Iiterature compared to literature+14) which had p-values sightly greater than 0.05 .

The hypothesis that the slopes are equal was not rejected $(0.05$ level of significance) for all tests except the following:

- Literature compared to K Basin "a 17 16"

- Literature compared to K Basin "edited 14"

- Literature compared to K Basin "best 7" 
Hypothesis testing to determine the equality of the intercepts was conducted only if the slopes had tested equal (the hypothesis that the slopes were equal was not rejected). The hypothesis that the intercepts are equa] was not rejected for these tests.

\subsection{SUMMARY OBSERVATIONS AND DISCUSSION}

Five of the sixteen $K$ Basin fuel data were not consistent with the literature data, falling well outside the $95 \%$ prediction intervals for the 7iterature data. At the high end of the temperature range $\left(400^{\circ} \mathrm{C}\right)$ the SNF datum was low, and all SNF data below $150^{\circ} \mathrm{C}$ were high in comparison to the literature data. Three of these five data were from "erratic" TGA curves and tested as statistical outlier data relative to the literature data. The remaining two data $\left(90^{\circ} \mathrm{C}\right.$ and $\left.69{ }^{\circ} \mathrm{C}\right)$ were from "normal" TGA curves.

The rate $T$ aw equations from regressions of the SNF data were significantly different than for the literature data. The $K$ Bas in SNF regression lines cross the literature data regression 1 ine at about $250{ }^{\circ} \mathrm{C}$ and cross the $95 \%$ upper prediction interval line somewhat above $150{ }^{\circ} \mathrm{C}$. This indicates higher oxidation rates at temperature below $150^{\circ} \mathrm{C}$ for $\mathrm{K}$ Basin SNF than would be Tikely to occur for unirradiated fuel.

It should be noted that the method for deriving linear rates from the SNF data was different than had been used for the literature data. This could account for differences between SNF oxidation rates and the literature rates. If, where possible, the more accepted way of evaluating oxidation data were used in evaluating the $K$ Basin fuel data, lower rates would, in some cases, be derived, and smaller differences would be observed between $K$ Basin fuel data and 1 iterature data. 
HNF-SD-SNF-CN-035, Rev. 1

\subsection{CONCLUSIONS}

The time-averaged linear oxidation rate data from $K$ Basin fuel tests in . dry air at temperatures from $150{ }^{\circ} \mathrm{C}$ to $350{ }^{\circ} \mathrm{C}$ were bounded by the $95 \%$ prediction intervals of the literature linear rate data for unirradiated uranium in dry air. Below $150^{\circ} \mathrm{C}$ K Basin SNF data were significantly greater than the Titerature data. Rate law equation from regressions of the SNF data predict higher rate at low temperatures (below $150^{\circ} \mathrm{C}$ ) than for unirradiated uranium. Above $250{ }^{\circ} \mathrm{C}$ SNF oxidation rate did not exceed rate for unirradiated uranium. 
HNF-SD-SNF-CN-035, Rev. 1

This page intentionally left blank. 


\subsection{REFERENCES}

Abrefah, J., H. C. Buchanan, W. M. Gerry, W. J. Gray, and S. C. Marschman, 1998, Dry Air Oxidation Kinetics of K Basin Spent Nuclear Fuel, PNNL-17786, Pacific Northwest National Laboratory, Richland, Washington.

ASTM, 1969, "General Test Methods," 1969 Book of ASTM Standards, Part 30, American Society for Testing and Materials, Philadelphia, Pennsylvania.

Pearce, R. J., 1989, "A Review of the Rates of Reaction of Unirradiated Uranium in Gaseous Atmosphere," RD/B/6231/R86, Central Electric Generating Board, Berkeley Nuclear Laboratories, United Kingdom.

Ritchie, A. G., 1984, "The Kinetics of the Initial Stages of the Reaction of Uranium with Oxygen," Journal of the Less-Common Meta7s, 98.

Snedecor, G. W. and W. G. Cochran, 1980, "Statistical Methods," Seventh Edition, Iowa State University Press, Ames, Iowa. 
HNF-SD-SNF-CN-035, Rev. 1

This page intentionally left blank. 
HNF-SD-SNF-CN-035, Rev. I

A P PENDIX A

DATA BASE FOR OXIDATION OF UNIRRADIATED URANIUM IN DRY AIR 
HNF-SD-SNF-CN-035, Rev. I

This page intentionally left blank. 
HNF-SD-SNF-CN-035, Rev. 1

\section{APPENDIX A}

\section{DATA BASE FOR OXIDATION OF UNIRRADIATED URANIUM IN DRY AIR}

The data for dry air oxidation of unirradiated uranium at temperatures less that $445^{\circ} \mathrm{C}$ are 1 isted in Table Al (Pearce 1989).

\section{REFERENCES}

Pearce, R. J., 1989, "A Review of the Rates of Reaction of Unirradiated Uranium in Gaseous Atmosphere," RD/B/6231/R86, Central Electric Generating Board, Berkeley Nuclear Laboratories, United Kingdom.

Waber, J. T., 1952, "A Review of the Corrosion of Uranium and Its Alloys," LA-1524, Los Alamos National Laboratory, Los Alamos, New Mexico. 
Table A1. Rate Data for Dry Air Oxidation of Unirradiated Uranium at Temperatures $\angle 445^{\circ} \mathrm{C}$ (Pearce 1989).

\begin{tabular}{|c|c|c|c|c|c|c|c|c|}
\hline \multicolumn{9}{|c|}{ PEARCE DATA } \\
\hline$T(C)$ & $1 / \mathrm{T}\left(\mathrm{K}^{-1}\right)$ & $\mathrm{mg} / \mathrm{cm}^{2} / \mathrm{hr}$ & $T(C)$ & $1 / \mathrm{T}\left(\mathrm{K}^{-1}\right)$ & $\mathrm{mg} / \mathrm{cm}^{2} / \mathrm{hr}$ & $T(\mathrm{C})$ & $1 / \pi\left(\mathrm{K}^{-1}\right)$ & $\mathrm{mg} / \mathrm{cm}^{2} / \mathrm{hr}$ \\
\hline $38^{*}$ & $3.24 \mathrm{E}-3$ & $5.0 \mathrm{E}-5$ & 192 & $2.15 \mathrm{E}-3$ & $7.0 \mathrm{E}-1$ & 259 & $1.88 \mathrm{E}-3$ & $1.6 \mathrm{E}+0$ \\
\hline 40 & $3.20 \mathrm{E}-3$ & $1.6 \mathrm{E}-5$ & 192 & $2.15 \mathrm{E}-3$ & $1.0 \mathrm{E}+0$ & 259 & $1.88 \mathrm{E}-3$ & $1.4 \mathrm{E}+0$ \\
\hline 40 & $3.20 \mathrm{E}-3$ & $2.0 \mathrm{E}-5$ & 196 & $2.13 \mathrm{E}-3$ & $1.1 \mathrm{E}+0$ & 262 & $1.87 \mathrm{E}-3$ & $1.8 E+0$ \\
\hline 40 & $3.20 \mathrm{E}-3$ & $3.0 \mathrm{E}-5$ & 199 & $2.12 E-3$ & $5.0 \mathrm{E}-2$ & 268 & $1.85 \mathrm{E}-3$ & $5.0 \mathrm{E}-1$ \\
\hline 50 & $3.10 \mathrm{E}-3$ & $3.1 \mathrm{E}-5$ & 199 & $2.12 \mathrm{E}-3$ & $1.0 \mathrm{E}-1$ & 268 & $1.85 \mathrm{E}-3$ & $1.6 \mathrm{E}+0$ \\
\hline 60 & $3.00 \mathrm{E}-3$ & $3.2 \mathrm{E}-5$ & 199 & $2.12 \mathrm{E}-3$ & $2.0 E-1$ & 268 & $1.85 \mathrm{E}-3$ & $1.3 \mathrm{E}+0$ \\
\hline 60 & $3.00 \mathrm{E}-3$ & $1.0 \mathrm{E}-4$ & 199 & $2.12 \mathrm{E}-3$ & $2.5 \mathrm{E}-1$ & 268 & $1.85 \mathrm{E}-3$ & $6.5 \mathrm{E}+0$ \\
\hline 60 & $3.00 \mathrm{E}-3$ & $1.5 \mathrm{E}-4$ & 201 & $2.11 \mathrm{E}-3$ & $6.3 \mathrm{E}-2$ & 270 & $1.84 \mathrm{E}-3$ & $1.6 \mathrm{E}+0$ \\
\hline 72 & $2.90 \mathrm{E}-3$ & $1.3 E-3$ & 201 & $2.11 \mathrm{E}-3$ & $1.0 \mathrm{E}-1$ & 273 & $1.83 \mathrm{E}-3$ & $9.0 \mathrm{E}+0$ \\
\hline 74 & $2.88 \mathrm{E}-3$ & 1.0E-5 & 201 & $2.11 \mathrm{E}-3$ & -1.5E-1 & 283 & $1.80 \mathrm{E}-3$ & $2.2 \mathrm{E}+0$ \\
\hline 96 & $2.71 \mathrm{E}-3$ & $6.6 \mathrm{E}-3$ & 201 & $2.11 \mathrm{E}-3$ & $1.3 \mathrm{E}-1$ & 289 & $1.78 \mathrm{E}-3$ & $2.5 \mathrm{E}+0$ \\
\hline 100 & $2.68 \mathrm{E}-3$ & 1.6E-4 & 201 & $2.11 \mathrm{E}-3$ & $1.6 \mathrm{E}-1$ & 289 & $1.78 \mathrm{E}-3$ & $4.5 \mathrm{E}+0$ \\
\hline 100 & $2.68 E-3$ & 7.0E-4 & 201 & $2.11 E-3$ & $2.0 \mathrm{E}-1$ & 289 & $1.78 \mathrm{E}-3$ & $1.6 \mathrm{E}+0$ \\
\hline 100 & $2.68 \mathrm{E}-3$ & $8.0 \mathrm{E}-4$ & 201 & $2.11 \mathrm{E}-3$ & 2.1E-1 & 289 & $1.78 \mathrm{E}-3$ & $2.1 \mathrm{E}+0$ \\
\hline 100 & $2.68 \mathrm{E}-3$ & $1.5 \mathrm{E}-3$ & 201 & $2.11 \mathrm{E}-3$ & $2.3 \mathrm{E}-1$ & 295 & $1.76 \mathrm{E}-3$ & $3.2 \mathrm{E}+0$ \\
\hline 100 & $2.68 \mathrm{E}-3$ & $1.8 \mathrm{E}-3$ & 201 & $2.11 \mathrm{E}-3$ & $2.5 \mathrm{E}-1$ & 295 & $1.76 E-3$ & $4.3 \mathrm{E}+0$ \\
\hline 100 & $2.68 \mathrm{E}-3$ & $2.5 \mathrm{E}-3$ & 201 & $2.11 \mathrm{E}-3$ & $2.9 \mathrm{E}-1$ & 295 & $1.76 \mathrm{E}-3$ & $5.0 \mathrm{E}+0$ \\
\hline 100 & $2.68 \mathrm{E}-3$ & $3.0 \mathrm{E}-3$ & 201 & $2.11 \mathrm{E}-3$ & $3.1 \mathrm{E}-1$ & 295 & $1.76 \mathrm{E}-3$ & $1.0 \mathrm{E}+1$ \\
\hline 100 & $2.68 \mathrm{E}-3$ & $3.5 \mathrm{E}-3$ & 201 & $2.11 \mathrm{E}-3$ & $4.8 \mathrm{E}-1$ & 298 & $1.75 \mathrm{E}-3$ & $2.5 \mathrm{E}+0$ \\
\hline 100 & $2.68 \mathrm{E}-3$ & $5.0 \mathrm{E}-3$ & 201 & $2.11 \mathrm{E}-3$ & $4.9 \mathrm{E}-1$ & 298 & $1.75 \mathrm{E}-3$ & $3.0 \mathrm{E}+0$ \\
\hline 104 & $2.65 \mathrm{E}-3$ & $3.5 \mathrm{E}-4$ & 201 & $2.11 \mathrm{E}-3$ & $1.0 \mathrm{E}+0$ & 298 & $1.75 \mathrm{E}-3$ & $4.0 \mathrm{E}+0$ \\
\hline 122 & $2.53 \mathrm{E}-3$ & $1.6 \mathrm{E}-3$ & 201 & $2.11 \mathrm{E}-3$ & $1.6 \mathrm{E}+0$ & 298 & $1.75 \mathrm{E}-3$ & $4.5 \mathrm{E}+0$ \\
\hline 125 & $2.51 \mathrm{E}-3$ & 8.0E-3 & 201 & $2.11 \mathrm{E}-3$ & $2.5 \mathrm{E}+0$ & 298 & $1.75 \mathrm{E}-3$ & $5.0 \mathrm{E}+0$ \\
\hline 125 & $2.51 \mathrm{E}-3$ & $1.4 \mathrm{E}-2$ & 201 & $2.11 \mathrm{E}-3$ & $4.0 \mathrm{E}+0$ & 305 & $1.73 \mathrm{E}-3$ & $2.0 \mathrm{E}+0$ \\
\hline 137 & $2.44 \mathrm{E}-3$ & $5.0 \mathrm{E}-3$ & 203 & $2.10 \mathrm{E}-3$ & $2.5 \mathrm{E}-2$ & 305 & $1.73 \mathrm{E}-3$ & $1.2 \mathrm{E}+1$ \\
\hline 151 & $2.36 \mathrm{E}-3$ & $5.8 \mathrm{E}-3$ & 203 & $2.10 \mathrm{E}-3$ & $5.0 \mathrm{E}-2$ & 308 & $1.72 E-3$ & $4.8 \mathrm{E}+0$ \\
\hline 151 & $2.36 \mathrm{E}-3$ & $6.0 \mathrm{E}-3$ & 203 & $2.10 \mathrm{E}-3$ & $6.5 \mathrm{E}-2$ & 312 & 1.71E-3 & $1.4 \mathrm{E}+1$ \\
\hline 151 & $2.36 \mathrm{E}-3$ & $1.2 \mathrm{E}-2$ & 203 & $2.10 \mathrm{E}-3$ & $9.0 \mathrm{E}-2$ & 322 & $1.68 \mathrm{E}-3$ & $7.0 \mathrm{E}+0$ \\
\hline 151 & $2.36 \mathrm{E}-3$ & 1.6E-2 & 203 & $2.10 \mathrm{E}-3$ & $4.0 \mathrm{E}-1$ & 326 & $1.67 \mathrm{E}-3$ & $2.5 \mathrm{E}+1$ \\
\hline 151 & $2.36 \mathrm{E}-3$ & $1.9 \mathrm{E}-2$ & 203 & $2,10 \mathrm{E}-3$ & $7.1 \mathrm{E}-1$ & 329 & $1.66 \mathrm{E}-3$ & $4.0 \mathrm{E}+0$ \\
\hline 151 & $2.36 \mathrm{E}-3$ & $2.0 \mathrm{E}-2$ & 208 & $2.08 \mathrm{E}-3$ & $5.0 \mathrm{E}-1$ & 329 & $1.66 \mathrm{E}-3$ & $8.0 \mathrm{E}+0$ \\
\hline 151 & $2.36 \mathrm{E}-3$ & $2.5 \mathrm{E}-2$ & 212 & $2.06 \mathrm{E}-3$ & $1.4 \mathrm{E}-1$ & 344 & $1.62 \mathrm{E}-3$ & $6.5 \mathrm{E}+0$ \\
\hline 151 & $2.36 \mathrm{E}-3$ & $3.0 \mathrm{E}-2$ & 220 & $2.03 \mathrm{E}-3$ & $5: 0 \mathrm{E}-1$ & 344 & $1.62 \mathrm{E}-3$ & $6.0 \mathrm{E}+1$ \\
\hline 151 & $2.36 \mathrm{E}-3$ & $5.0 \mathrm{E}-2$ & 225 & $2.01 \mathrm{E}-3$ & $8.0 \mathrm{E}-1$ & 348 & $1.61 \mathrm{E}-3$ & $9.0 \mathrm{E}+0$ \\
\hline 151 & $2.36 E-3$ & $6.0 \mathrm{E}-2$ & 227 & $2.00 \mathrm{E}-3$ & $9.0 \mathrm{E}-2$ & 360 & $1.58 \mathrm{E}-3$ & $1.6 \mathrm{E}+1$ \\
\hline 151 & $2.36 \mathrm{E}-3$ & $9.0 \mathrm{E}-2$ & 227 & $2.00 \mathrm{E}-3$ & 1.6E-1 & 360 & $1.58 \mathrm{E}-3$ & $5.0 \mathrm{E}+1$ \\
\hline 151 & $2.36 \mathrm{E}-3$ & $3.2 \mathrm{E}-1$ & 227 & $2.00 E-3$ & $4.0 \mathrm{E}-1$ & 360 & $1.58 \mathrm{E}-3$ & $8.0 \mathrm{E}+0$ \\
\hline 158 & $2.32 \mathrm{E}-3$ & $1.3 \mathrm{E}-2$ & 227 & $2.00 \mathrm{E}-3$ & $5.0 \mathrm{E}-1$ & 372 & $1.55 \mathrm{E}-3$ & $1.6 \mathrm{E}+1$ \\
\hline 160 & $2.31 \mathrm{E}-3$ & $1.6 \mathrm{E}-2$ & 227 & $2.00 \mathrm{E}-3$ & $6.5 E-1$ & 385 & $1.52 \mathrm{E}-3$ & 1.3E+1 \\
\hline 160 & $2.31 \mathrm{E}-3$ & 3.3E-2 & 227 & $2.00 \mathrm{E}-3$ & $8.0 \mathrm{E}-1$ & 389 & $1.51 \mathrm{E}-3$ & $2.5 \mathrm{E}+1$ \\
\hline 168 & $2.27 \mathrm{E}-3$ & $1.0 \mathrm{E}-1$ & 230 & $1.99 \mathrm{E}-3$ & $4.5 \mathrm{E}-1$ & 389 & $1.51 \mathrm{E}-3$ & $3.3 E+1$ \\
\hline 169 & $2.26 \mathrm{E}-3$ & 4.0E-2 & 230 & 1.99E-3 & $1.3 E+0$ & 389 & $1.51 \mathrm{E}-3$ & $6.0 \mathrm{E}+1$ \\
\hline 169 & $2.26 \mathrm{E}-3$ & 5.0E-2 & 235 & $1.97 \mathrm{E}-3$ & $3.5 \mathrm{E}-1$ & 389 & $1.51 \mathrm{E}-3$ & $8.0 \mathrm{E}+1$ \\
\hline 169 & $2.26 \mathrm{E}-3$ & 1.0E-1 & 237 & $1.96 \mathrm{E}-3$ & $6.5 \mathrm{E}-1$ & 389 & $1.51 \mathrm{E}-3$ & $1.0 \mathrm{E}+2$ \\
\hline 179 & $2.21 E-3$ & $1.6 \mathrm{E}-2$ & 237 & $1.96 \mathrm{E}-3$ & $1.1 E+0$ & 394 & $1.50 \mathrm{E}-3$ & $5.0 \mathrm{E}+1$ \\
\hline 179 & $2.21 \mathrm{E}-3$ & $4.5 \mathrm{E}-2$ & 245 & $1.93 \mathrm{E}-3$ & $8.0 \mathrm{E}-1$ & 394 & $1.50 \mathrm{E}-3$ & $5.8 \mathrm{E}+1$ \\
\hline 179 & $2.21 \mathrm{E}-3$ & $5.0 \mathrm{E}-2$ & 251 & $1.91 \mathrm{E}-3$ & $1.1 \mathrm{E}+0$ & 394 & $1.50 \mathrm{E}-3$ & $3.8 E+1$ \\
\hline 179 & $2.21 \mathrm{E}-3$ & $1.0 \mathrm{E}-1$ & 251 & $1.91 \mathrm{E}-3$ & $1.6 \mathrm{E}+0$ & 398 & $1.49 \mathrm{E}-3$ & $2.0 \mathrm{E}+1$ \\
\hline 179 & $2.21 \mathrm{E}-3$ & $5.0 \mathrm{E}-1$ & 251 & 1.91E-3 & $1.9 \mathrm{E}+0$ & 398 & $1.49 \mathrm{E}-3$ & $3.0 \mathrm{E}+1$ \\
\hline 179 & $2.21 \mathrm{E}-3$ & 1.5E-1 & 253 & $1.90 \mathrm{E}-3$ & $4.0 \mathrm{E}-1$ & 398 & $1.49 \mathrm{E}-3$ & $3.2 \mathrm{E}+1$ \\
\hline 182 & $2.20 \mathrm{E}-3$ & $4.0 \mathrm{E}-2$ & 253 & $1.90 \mathrm{E}-3$ & $8.0 \mathrm{E}-1$ & 398 & $1.49 \mathrm{E}-3$ & $4.0 \mathrm{E}+1$ \\
\hline 188 & $2.17 E-3$ & 1.0E-1 & 253 & $1.90 \mathrm{E}-3$ & $1.0 \mathrm{E}+0$ & 398 & $1.49 \mathrm{E}-3$ & $1.0 \mathrm{E}+2$ \\
\hline 188 & $2.17 \mathrm{E}-3$ & $1.6 \mathrm{E}-1$ & 253 & $1.90 \mathrm{E}-3$ & $1.4 \mathrm{E}+0$ & 441 & $1.40 \mathrm{E}-3$ & 1.0E+2 \\
\hline 192 & $2.15 \mathrm{E}-3$ & $4.0 \mathrm{E}-2$ & 259 & $1.88 \mathrm{E}-3$ & $1.1 \mathrm{E}+0$ & & & \\
\hline
\end{tabular}


HNF-SD-SNF-CN-035, Rev. 1

A P PEN DIX B

WEIGHT GAIN DATA PLOTS WITH RATE ANALYSIS DATA FROM OXIDATION TESTING OF $K$ BASIN FUEL SAMPLES IN DRY AIR 
HNF-SD-SNF-CN-035, Rev. I

This page intentionally left blank. 
Figure B1. K Basin Fue1 Sample Run 6 at $200^{\circ} \mathrm{C}$.
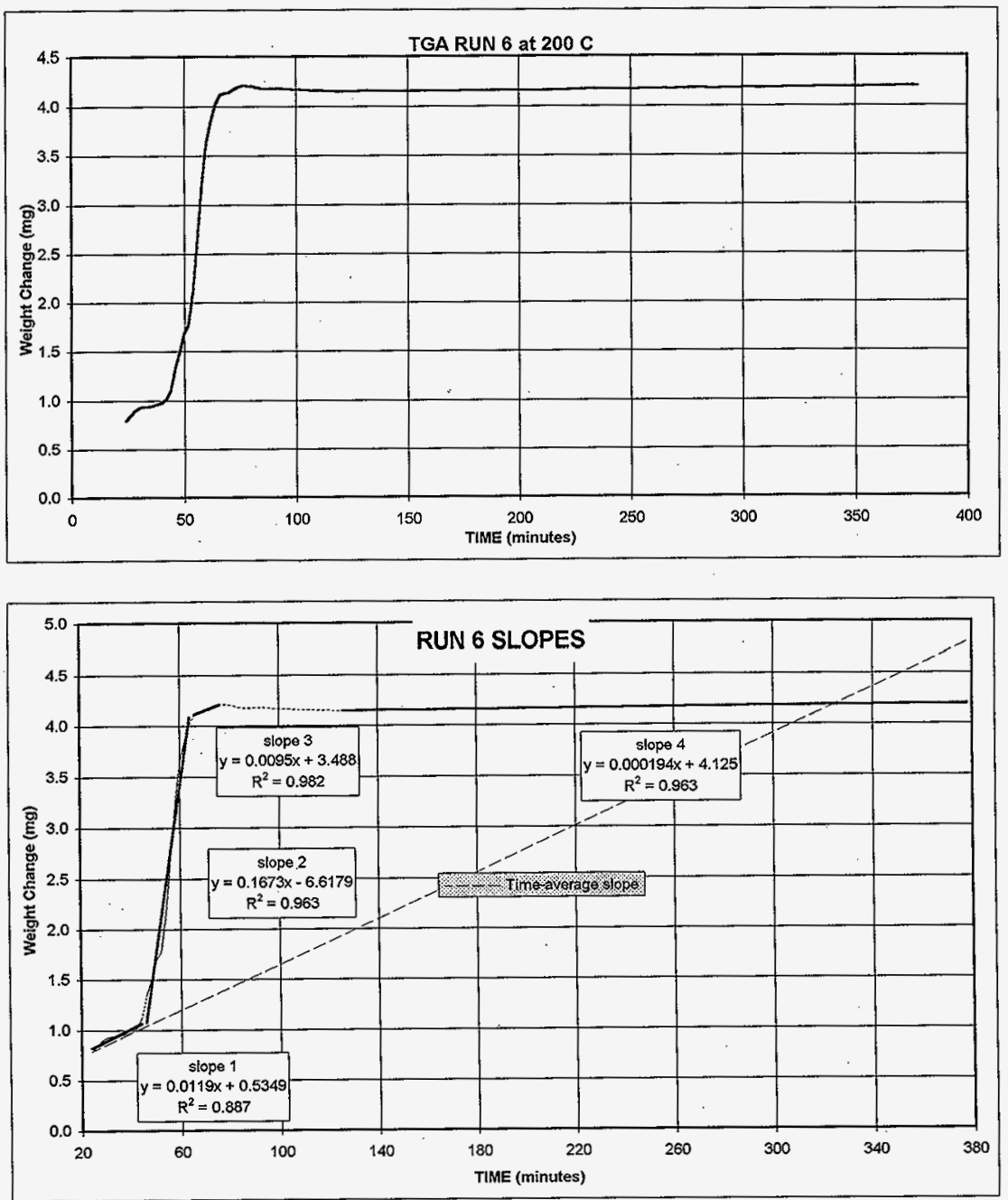
Figure B2. $\mathrm{K}$ Basin Fuel Sample Run $6 r$ at $200{ }^{\circ} \mathrm{C}$.
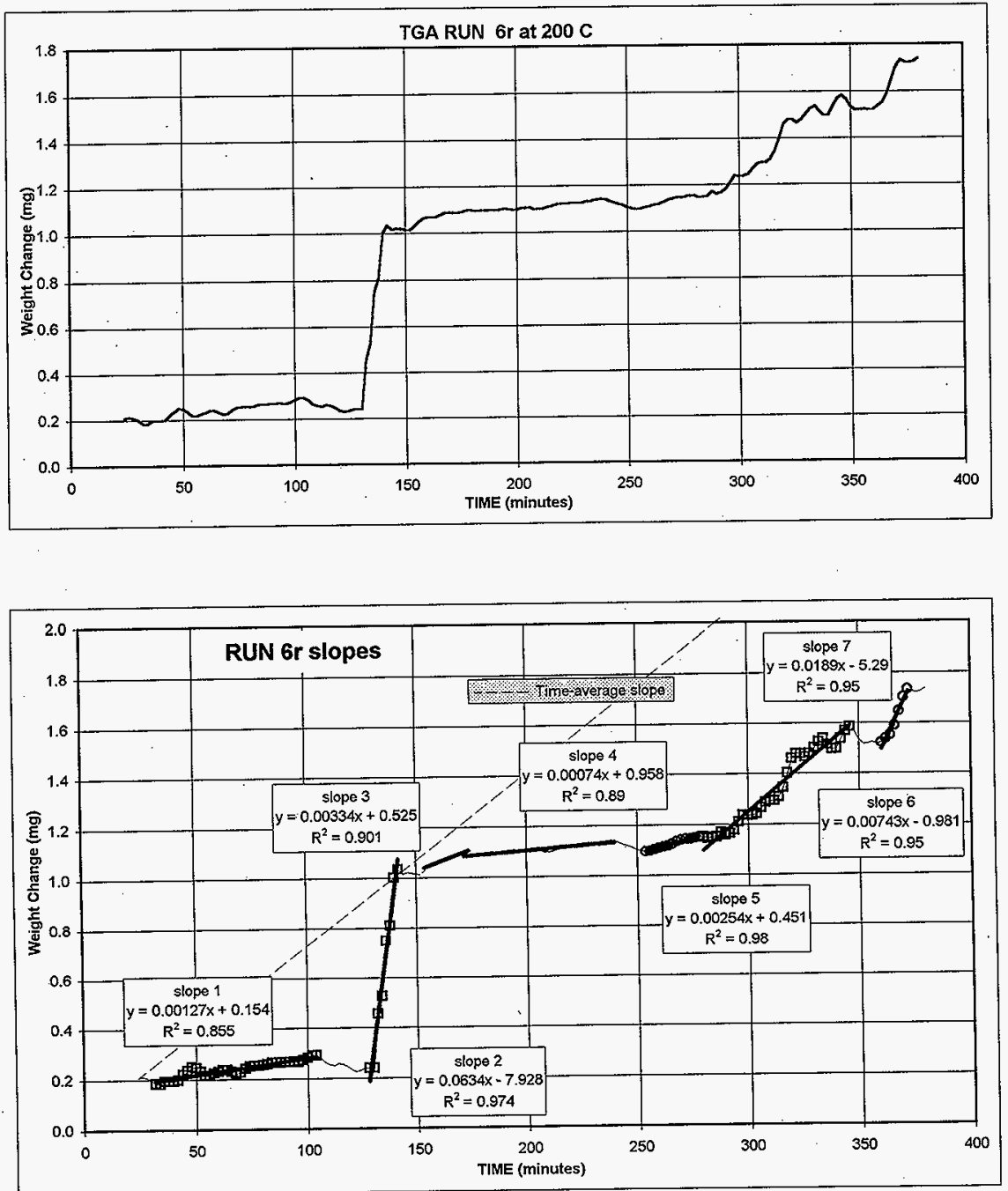
HNF-SD-SNF-CN-035, Rev. 1

Figure B3. K Basin Fuel Sample Run 7 at $300{ }^{\circ} \mathrm{C}$.
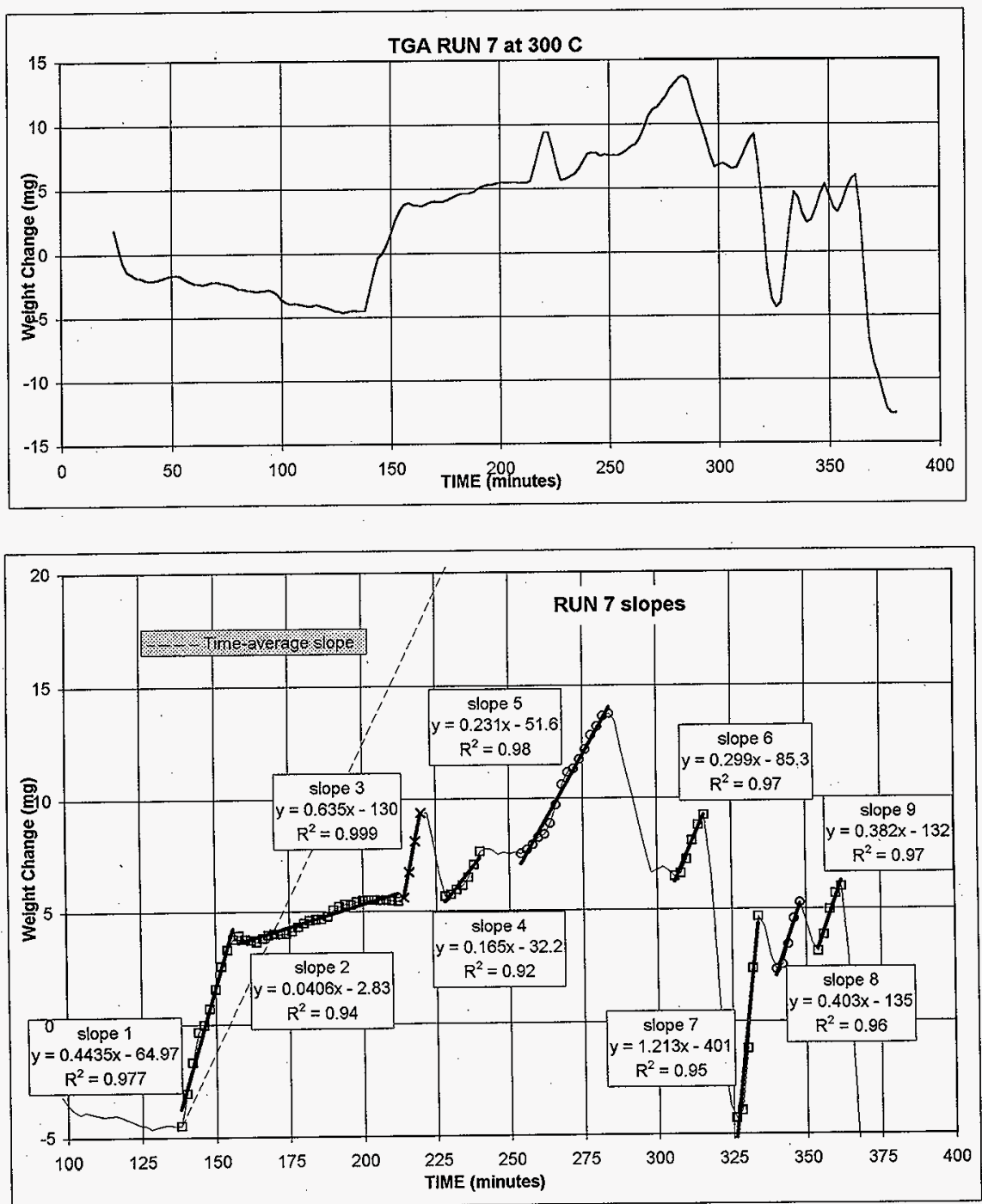
HNF-SD-SNF-CN-035, Rev. 1

Figure B4. K Basin Fuel Sample Run 8 at $400^{\circ} \mathrm{C}$.
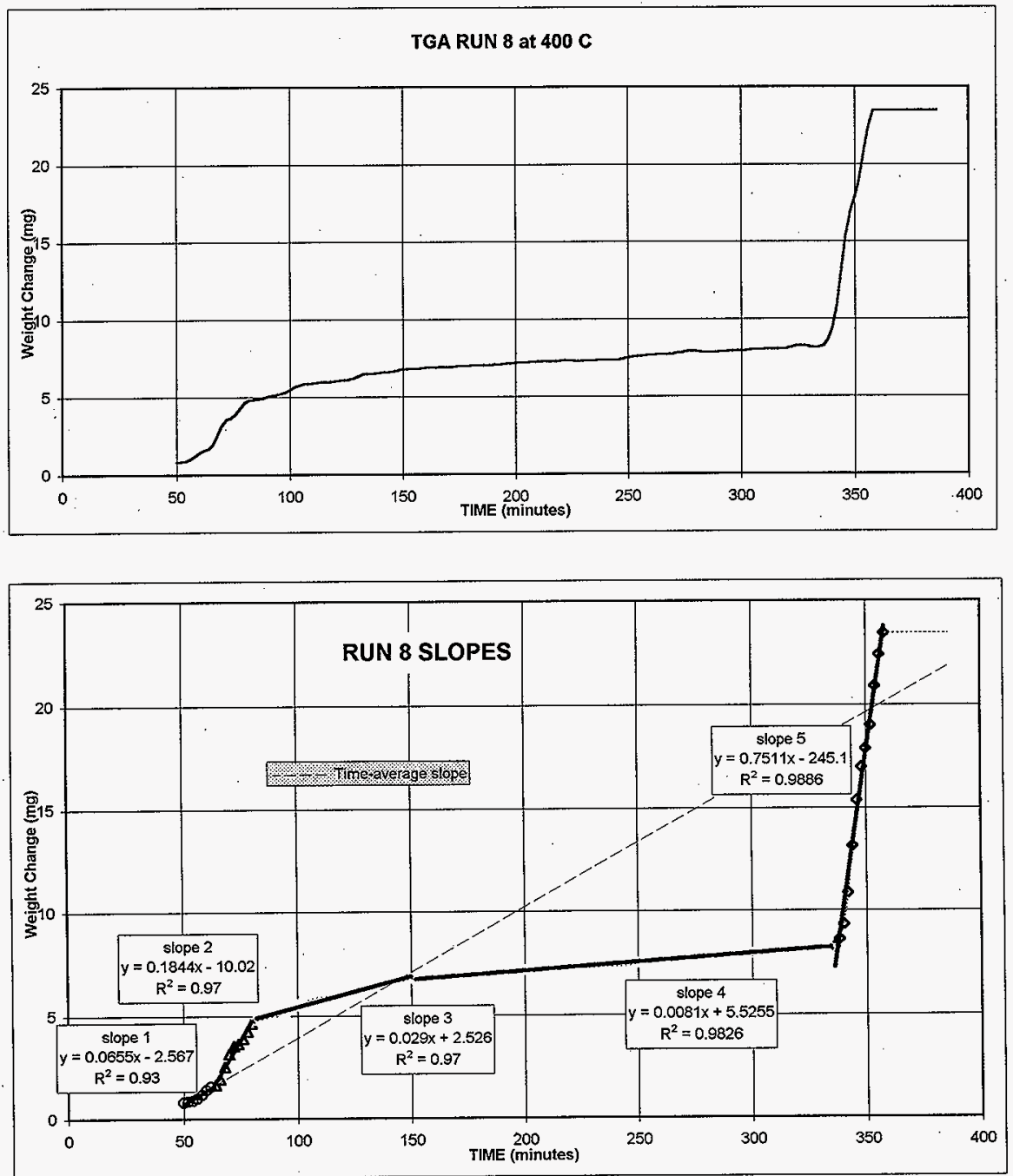
Figure B5. K Basin Fuel Sample Run 18 at $144{ }^{\circ} \mathrm{C}$.

TGA RUN 18 at $144 \mathrm{C}$
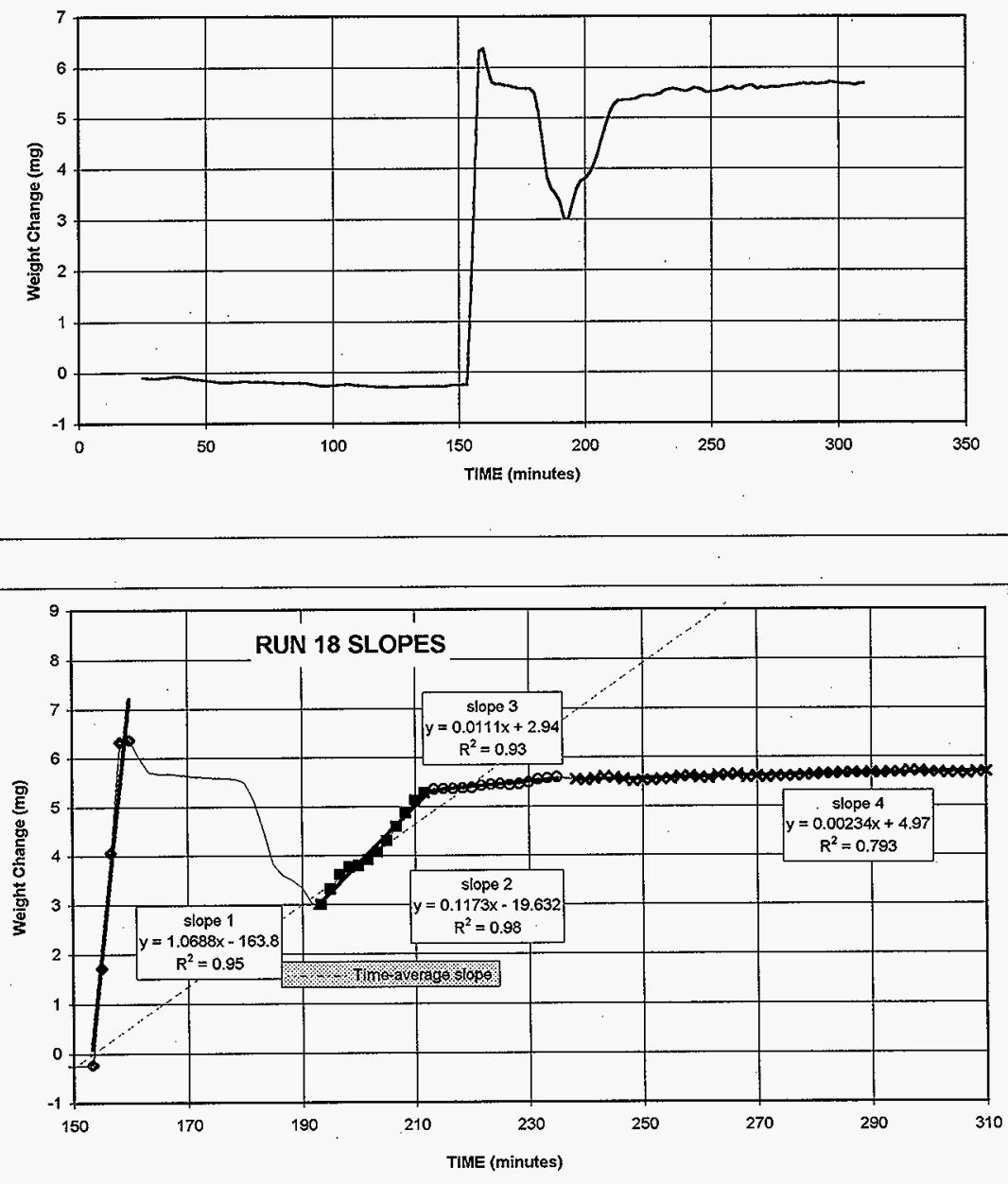
Figure B6. K Basin Fuel Sample Run $18 \mathrm{~b}$ at $150^{\circ} \mathrm{C}$.
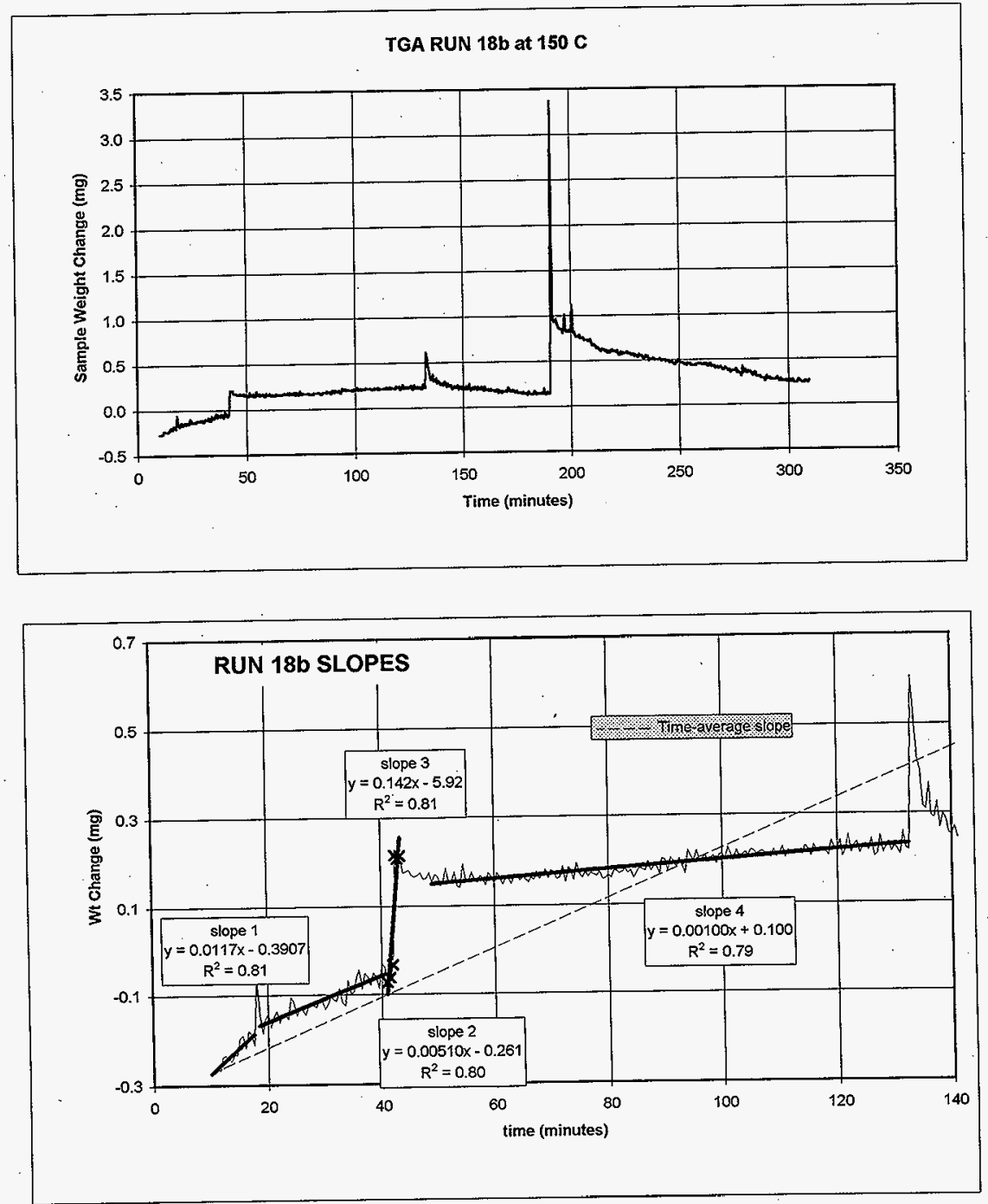
Figure B7. K Basin Fuel Sample Run $19 \mathrm{a}$ at $200{ }^{\circ} \mathrm{C}$.
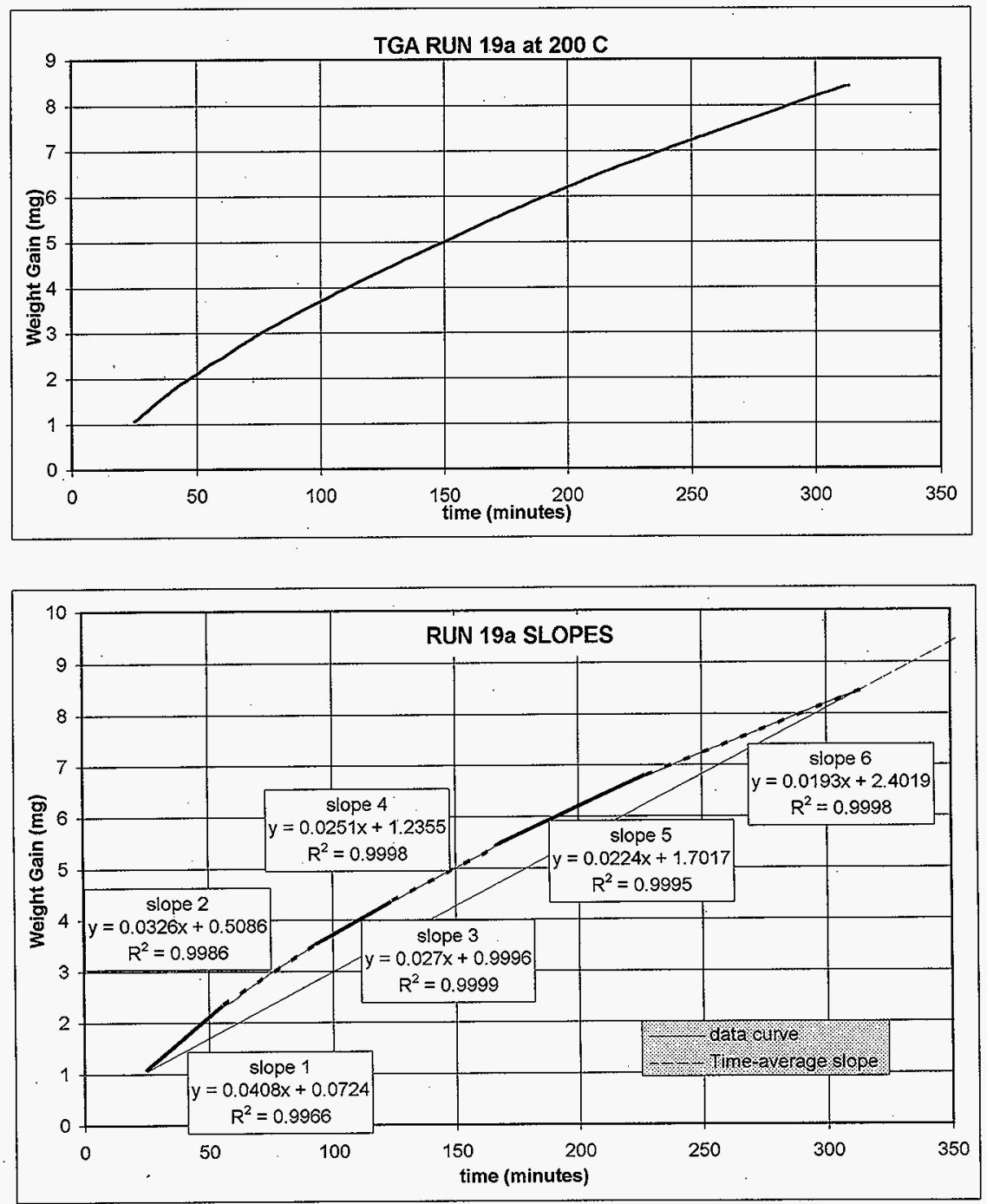
Figure B8. K Basin Fuel Sample Run 20 at $250{ }^{\circ} \mathrm{C}$.
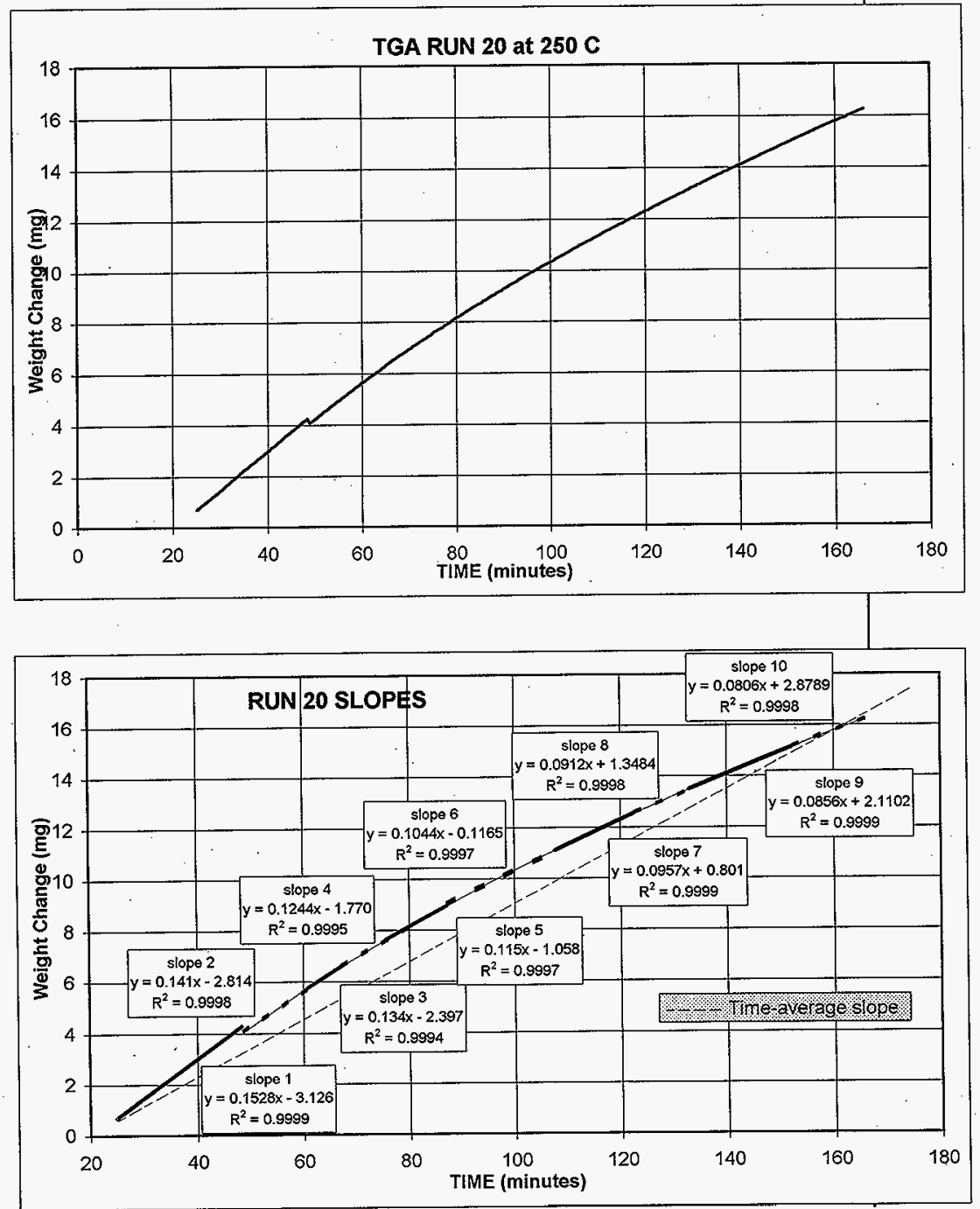
Figure B9. K Basin Fuel Sample Run 2la at $300{ }^{\circ} \mathrm{C}$.
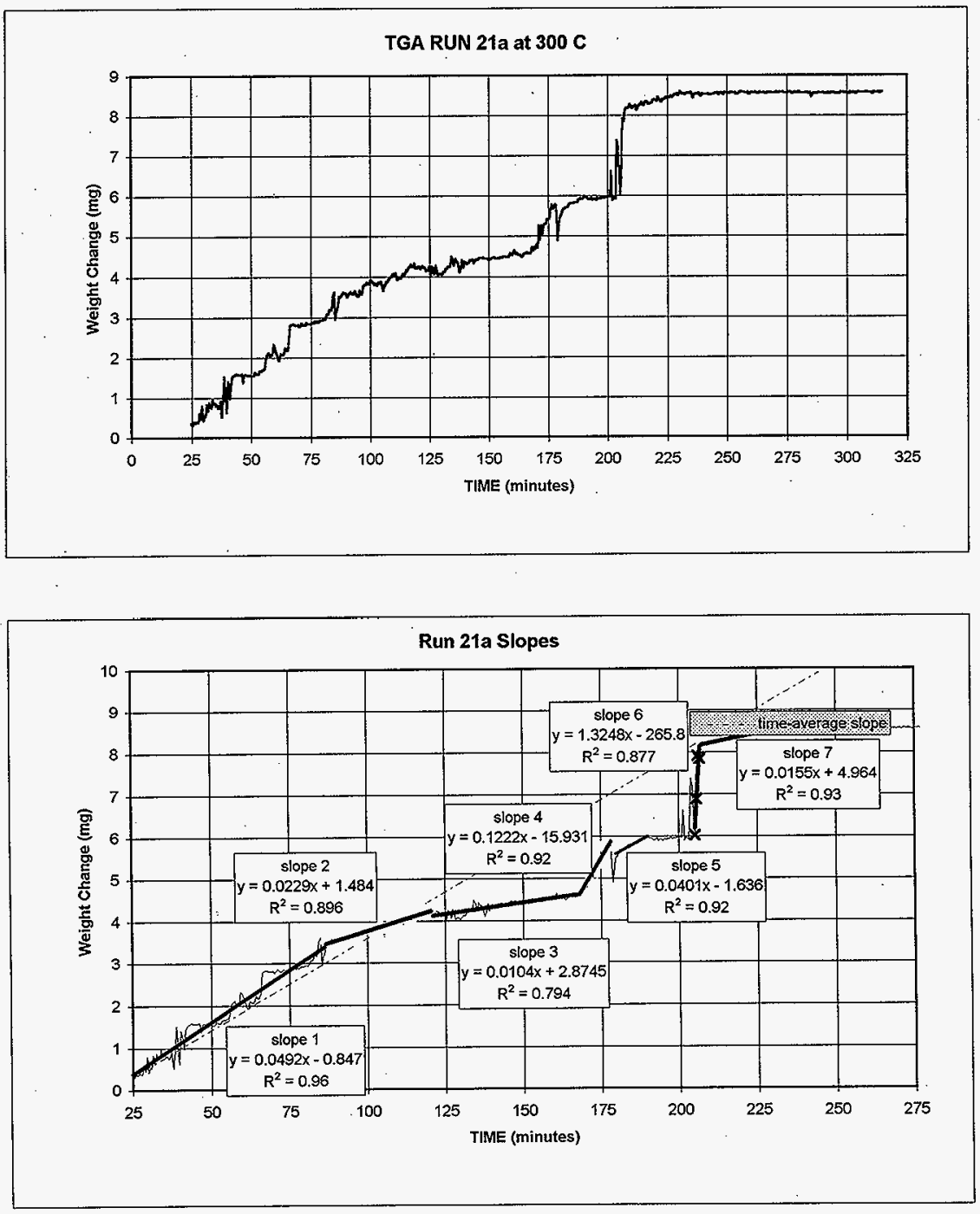
Figure B10. K Basin Fuel Sample Run 22 at $350{ }^{\circ} \mathrm{C}$.

TGA RUN 22 at $350 \mathrm{C}$
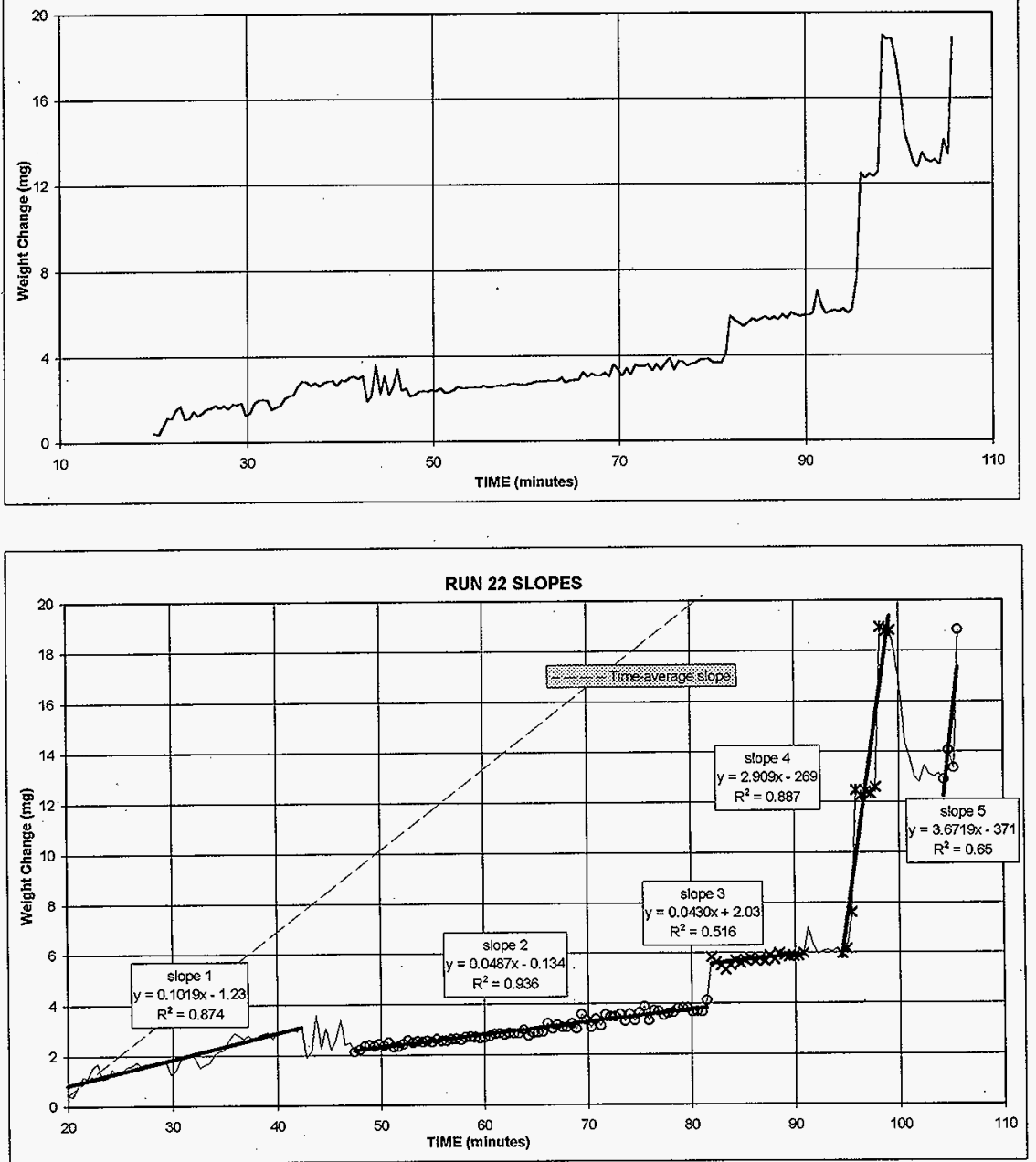
Figure B11. K Basin Fuel Sample Run 23 at $150^{\circ} \mathrm{C}$. TGA RUN 23 at $150 \mathrm{C}$

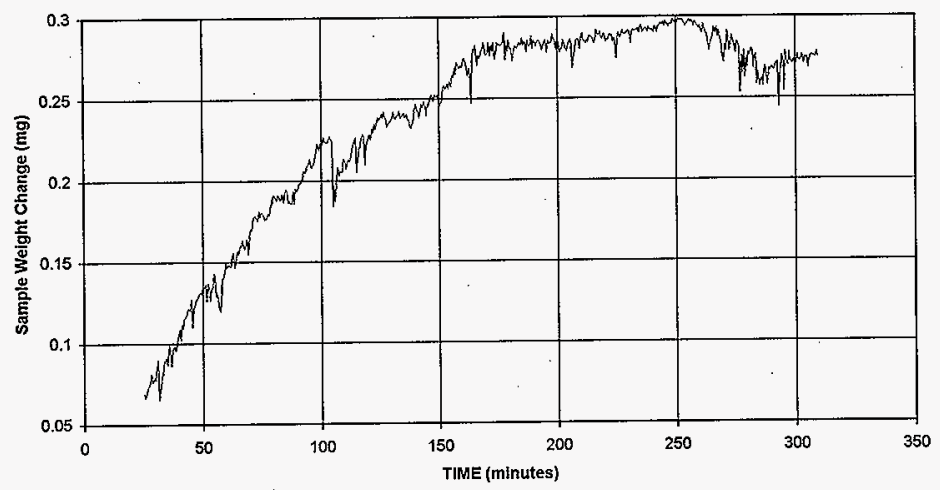

TGA RUN 23 SLOPES

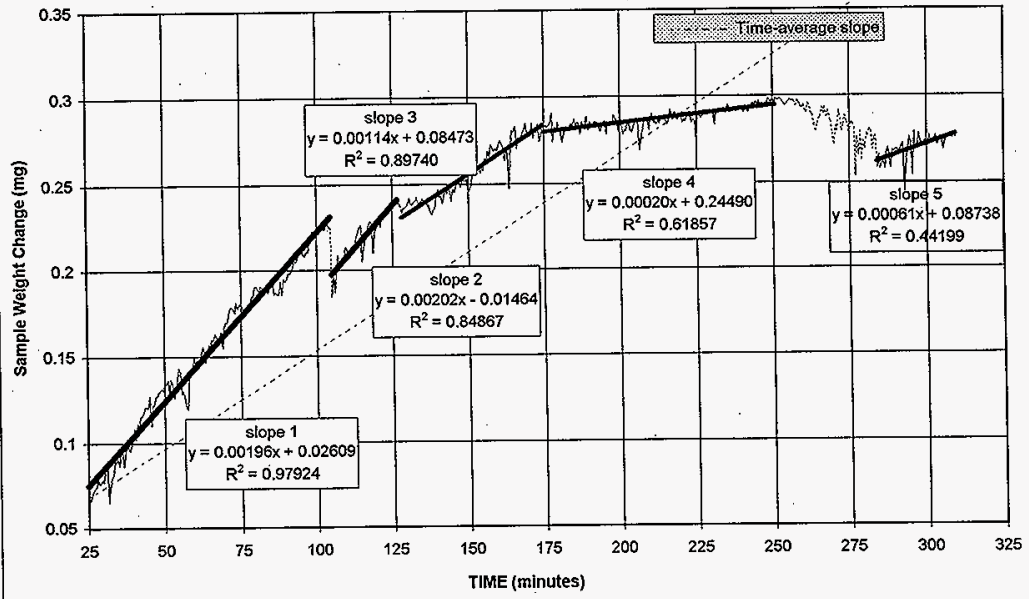


Figure B12. K Basin Fuel Sample Run 47 at $225^{\circ} \mathrm{C}$.
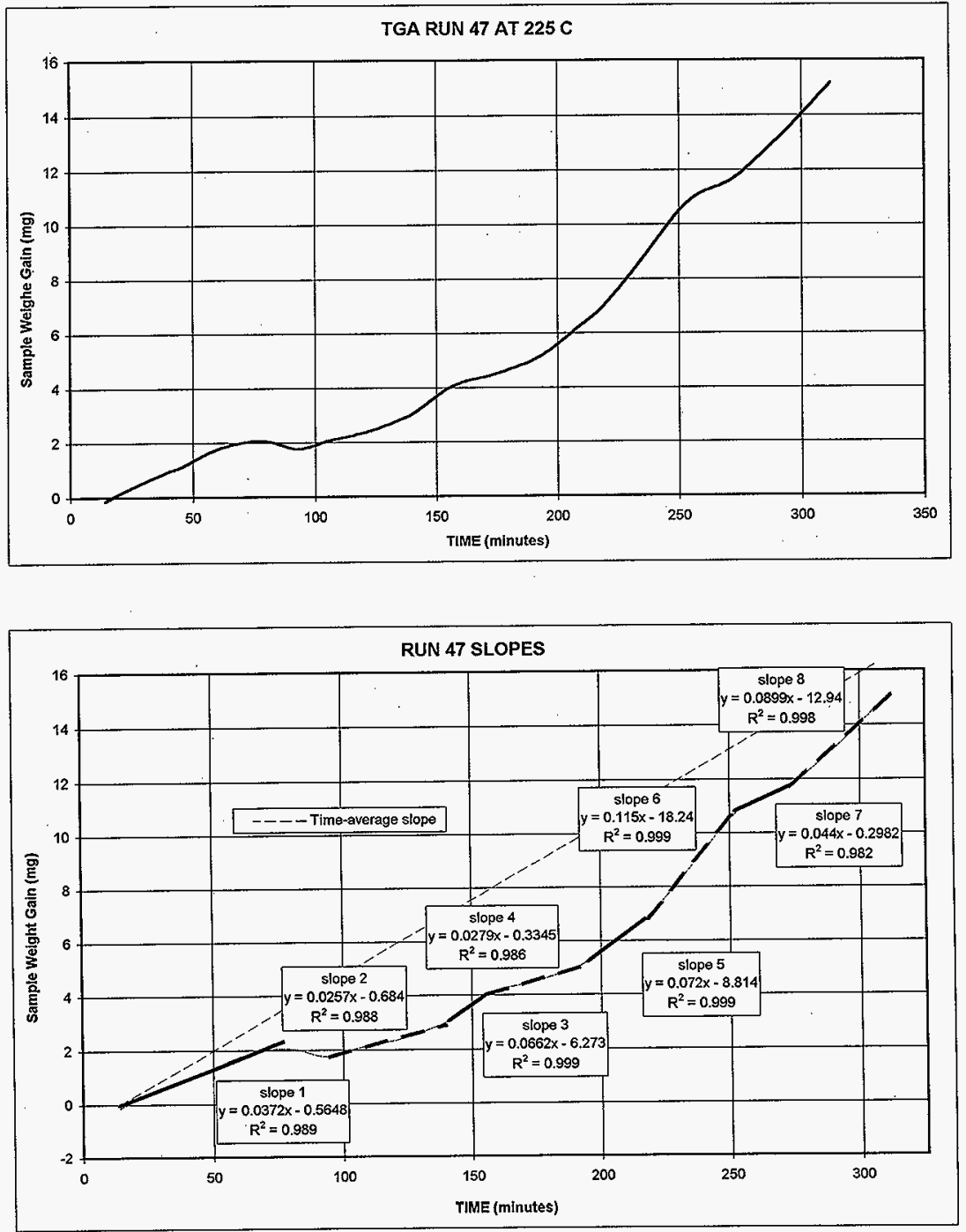
Figure B13. K Basin Fuel Sample Run 48 at $275^{\circ} \mathrm{C}$.
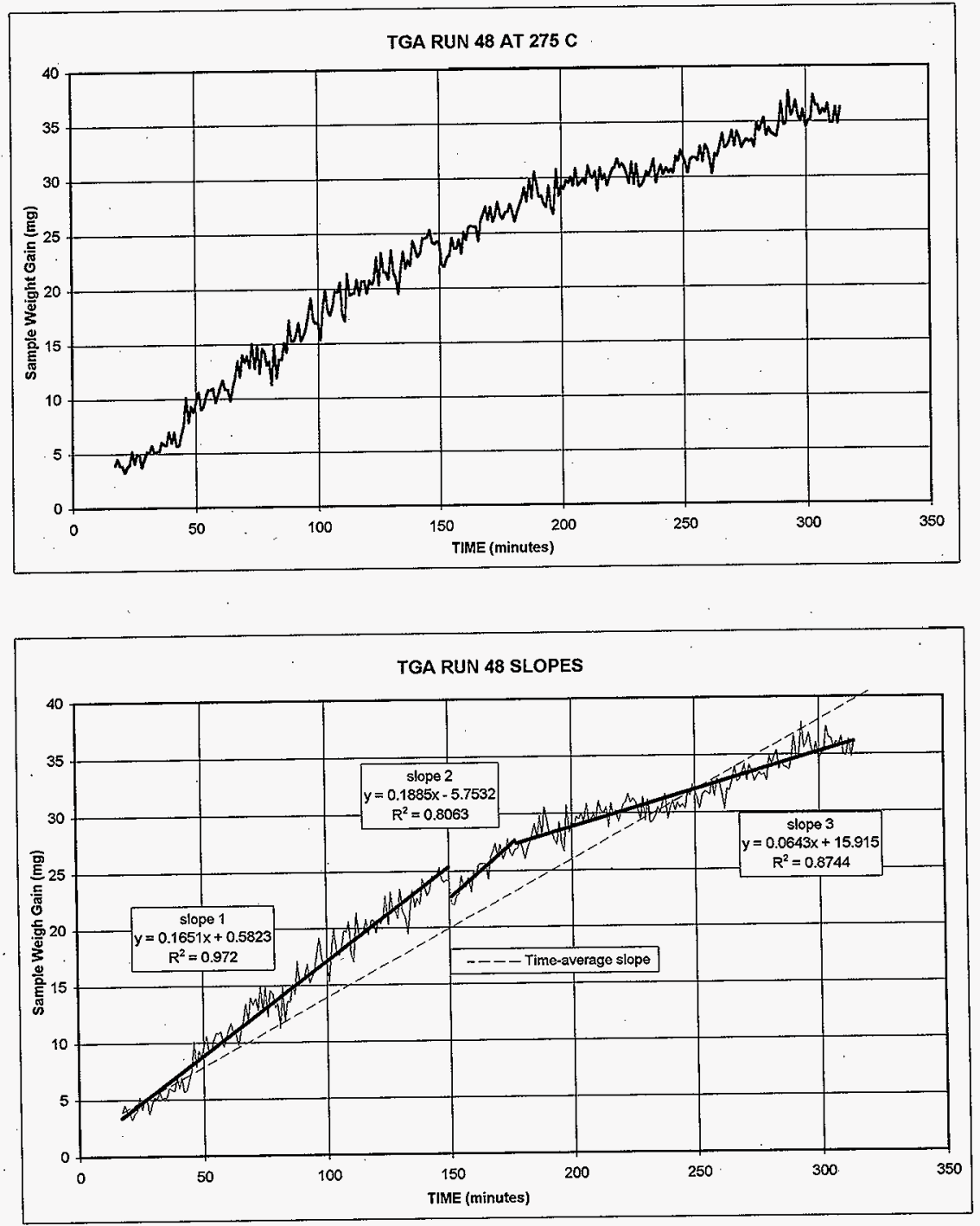
Figure B14. K Basin Fuel Sample Run $49 \mathrm{a}$ at $90^{\circ} \mathrm{C}$.
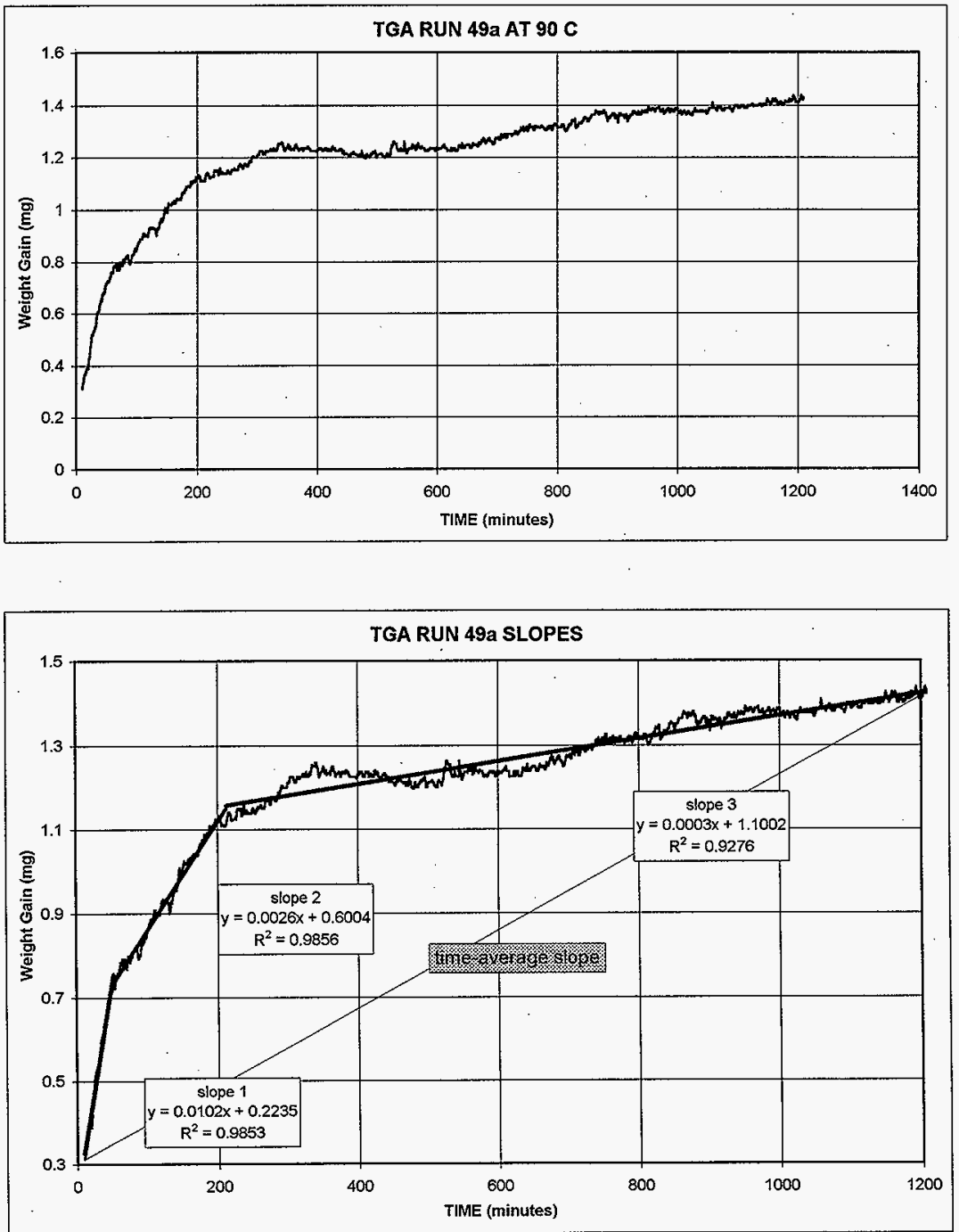
HNF-SD-SNF-CN-035, Rev. 1

Figure B15. K Basin Fuel Sample Run $49 \mathrm{~b}$ at $124{ }^{\circ} \mathrm{C}$.
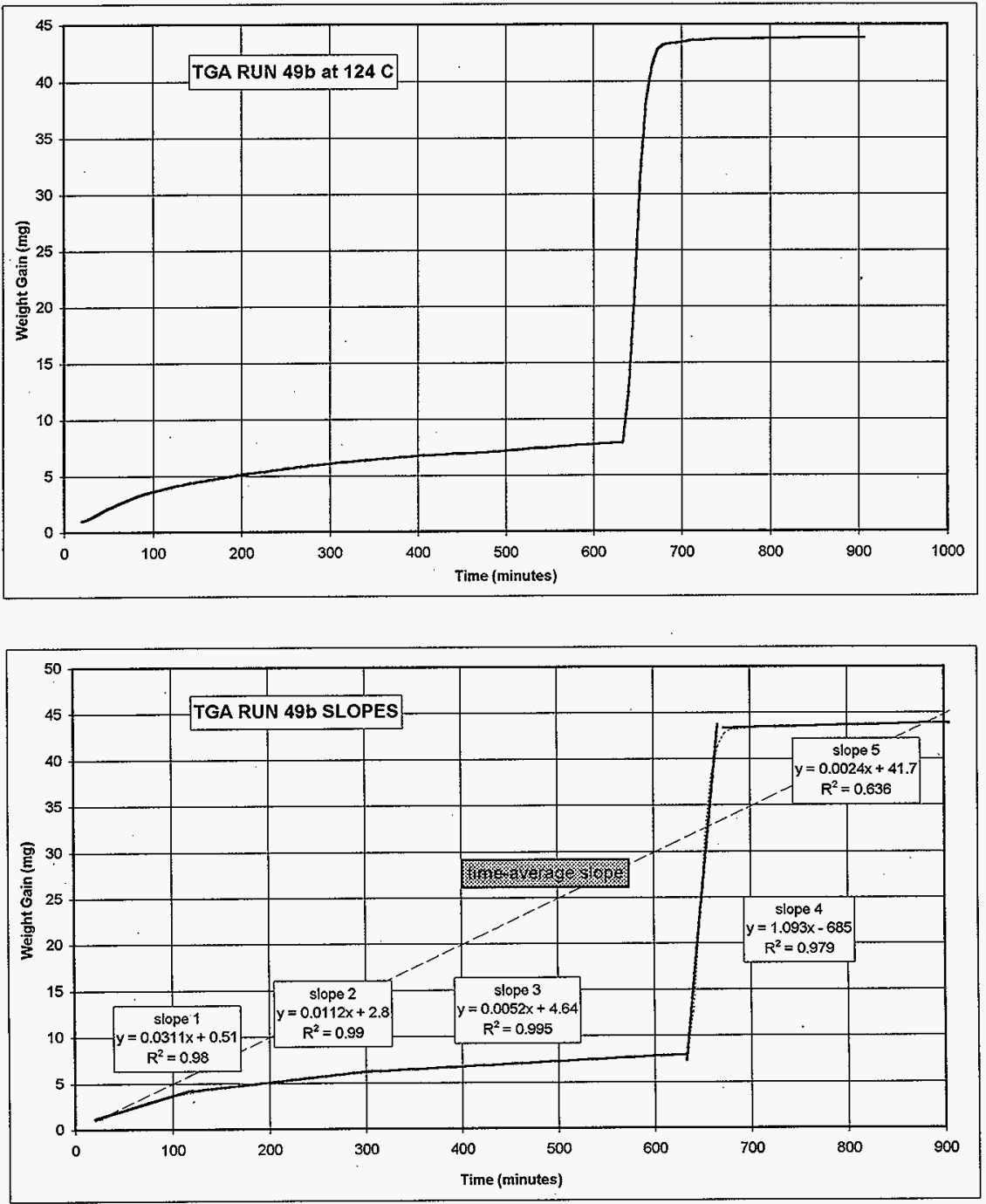
HNF-SD-SNF-CN-035, Rev. 1

Figure B16. K Basin Fuel Sample Run 51 at $69{ }^{\circ} \mathrm{C}$.
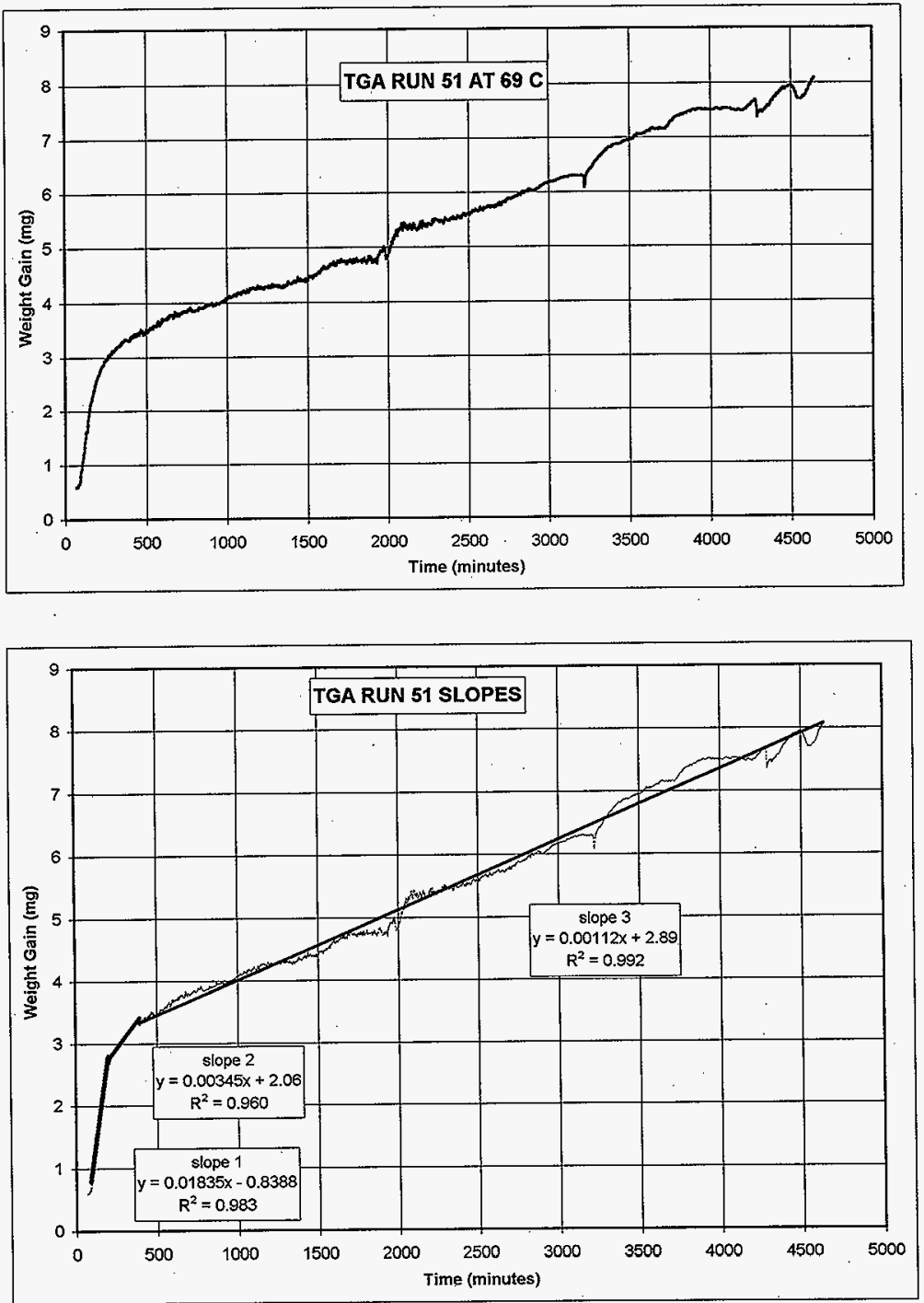
HNF-SD-SNF-CN-035, Rev. 1

This page intentionally left blank. 
HNF-SD-SNF-CN-035, Rev. 1

A P PENDIX C

WEIGHT GAIN DATA TABLES FROM OXIDATION TESTING

OF $K$ BASIN FUEL SAMPLES IN DRY AIR 
HNF-SD-SNF-CN-035, Rev. 1

This page intentionally left blank. 
Table C1. Thermo-Gravimetric Analysis Data

for $K$ Basin Fuel Sample Run 6 at $200{ }^{\circ} \mathrm{C}$.

\begin{tabular}{|c|c|c|c|c|c|c|c|}
\hline \multicolumn{8}{|c|}{ RUN NUMBER 6 AT $200 \mathrm{C}$} \\
\hline $\begin{array}{c}\text { time } \\
\text { minutes }\end{array}$ & $\begin{array}{c}\text { wt gain } \\
\mathrm{mg}\end{array}$ & $\begin{array}{c}\text { time } \\
\text { minutes }\end{array}$ & $\begin{array}{c}\text { wt gain } \\
\text { mg }\end{array}$ & $\begin{array}{c}\text { time } \\
\text { minutes }\end{array}$ & $\begin{array}{c}\text { wt gain } \\
\text { mg }\end{array}$ & $\begin{array}{c}\text { time } \\
\text { minutes }\end{array}$ & $\begin{array}{c}\text { wit gain } \\
\text { mg }\end{array}$ \\
\hline 24.00 & 0.7912 & 120.00 & 4.1525 & 216.00 & 4.1613 & 312.00 & 4.1821 \\
\hline 26.00 & 0.8429 & 122.00 & 4.1504 & 218.00 & 4.1617 & 314.00 & 4.1825 \\
\hline 28.00 & 0.8950 & 124.00 & 4.1496 & 220.00 & 4.1629 & 316.00 & 4.1833 \\
\hline 30.00 & 0.9262 & 126.00 & 4.1492 & 222.00 & 4.1642 & 318.00 & 4.1846 \\
\hline 32.00 & 0.9375 & 128.00 & 4.1542 & 224.00 & 4.1654 & 320.00 & 4.1854 \\
\hline 34.00 & 0.9379 & 130.00 & 4.1567 & 226.00 & 4.1679 & 322.00 & 4.1863 \\
\hline 36.00 & 0.9471 & 132.00 & 4.1583 & 228.00 & 4.1713 & 324.00 & 4.1875 \\
\hline 38.00 & 0.9629 & 134.00 & 4.1567 & 230.00 & 4.1754 & 326.00 & 4.1883 \\
\hline 40.00 & 0.9792 & 136.00 & 4.1554 & 232.00 & 4.1767 & 328.00 & 4.1888 \\
\hline 42.00 & 1.0175 & 138.00 & 4.1542 & 234.00 & 4.1758 & 330.00 & 4.1888 \\
\hline 44.00 & 1.1096 & 140.00 & 4.1529 & 236.00 & 4.1725 & 332.00 & 4.1892 \\
\hline 46.00 & 1.3392 & 142.00 & 4.1533 & 238.00 & 4.1704 & 334.00 & 4.1896 \\
\hline 48.00 & 1.5004 & 144.00 & 4.1546 & 240.00 & 4.1700 & 336.00 & 4.1904 \\
\hline 50.00 & 1.6979 & 146.00 & 4.1563 & 242.00 & 4.1713 & 338.00 & 4.1908 \\
\hline 52.00 & 1.7817 & 148.00 & 4.1571 & 244.00 & 4.1708 & 340.00 & 4.1913 \\
\hline 54.00 & 2.1125 & 150.00 & 4.1579 & 246.00 & 4.1704 & 342.00 & 4.1913 \\
\hline 56.00 & 2.6500 & 152.00 & 4.1575 & 248.00 & 4.1696 & 344.00 & 4.1917 \\
\hline 58.00 & 3.2150 & 154.00 & 4.1579 & 250.00 & 4.1704 & 346.00 & 4.1929 \\
\hline 60.00 & 3.6433 & 156.00 & 4.1583 & 252.00 & 4.1721 & 348.00 & 4.1933 \\
\hline 62.00 & 3.8542 & 158.00 & 4.1588 & 254.00 & 4.1737 & 350.00 & 4.1938 \\
\hline 64.00 & 4.0233 & 160.00 & 4.1571 & 256.00 & 4.1758 & 352.00 & 4.1933 \\
\hline 66.00 & 4.1200 & 162.00 & 4.1558 & 258.00 & 4.1758 & 354.00 & 4.1950 \\
\hline 68.00 & 4.1329 & 164.00 & 4,1554 & 260.00 & 4.1754 & 356.00 & 4.1963 \\
\hline 70.00 & 4.1454 & 166.00 & 4.1546 & 262.00 & 4.1746 & 358.00 & 4.1979 \\
\hline 72.00 & 4.1688 & 168.00 & 4.1538 & 264.00 & 4.1742 & 360.00 & 4.1979 \\
\hline 74.00 & 4.1954 & 170.00 & 4.1533 & 266.00 & 4.1742 & 362.00 & 4.1979 \\
\hline 76.00 & 4.2108 & 172.00 & 4.1558 & 268.00 & 4.1733 & 364.00 & 4.1979 \\
\hline 78.00 & 4.2121 & 174.00 & 4.1583 & 270.00 & 4.1738 & 366.00 & 4.1988 \\
\hline 80.00 & 4.2050 & 176.00 & 4.1592 & 272.00 & 4.1750 & 368.00 & 4.2000 \\
\hline 82.00 & 4.1963 & 178.00 & 4.1583 & 274.00 & 4.1779 & 370.00 & 4.2008 \\
\hline 84.00 & 4.1842 & 180.00 & 4.1583 & 276.00 & 4.1804 & 372.00 & 4.2021 \\
\hline 86.00 & 4.1800 & 182.00 & 4.1592 & 278.00 & 4.1812 & 374.00 & 4.2013 \\
\hline 88.00 & 4.1788 & 184.00 & 4.1608 & 280.00 & 4.1813 & 376.00 & 4.1992 \\
\hline 90.00 & 4.1825 & 186.00 & 4.1613 & 282.00 & 4.1804 & 378.00 & 4.1963 \\
\hline 92.00 & 4.1821 & 188.00 & 4.1608 & 284.00 & 4.1796 & & \\
\hline 94.00 & 4.1808 & 190.00 & 4.1613 & 286.00 & 4.1783 & & \\
\hline 96.00 & 4.1775 & 192.00 & 4.1613 & 288.00 & 4.1779 & & \\
\hline 98.00 & 4.1754 & 194.00 & 4.1621 & 290.00 & 4.1788 & & \\
\hline 100.00 & 4.1725 & 196.00 & 4.1625 & 292.00 & 4.1800 & & \\
\hline 102.00 & 4.1700 & 198.00 & 4.1617 & 294.00 & 4.1812 & & \\
\hline 104.00 & 4.1657 & 200.00 & 4.1617 & 296.00 & 4.1821 & & \\
\hline 106.00 & 4.1633 & 202.00 & 4.1608 & 298.00 & 4.1833 & & \\
\hline 108.00 & 4.1608 & 204.00 & 4.1617 & 300.00 & 4.1838 & & \\
\hline 110.00 & 4.1596 & 206.00 & 4.1617 & 302.00 & 4.1850 & & \\
\hline 112.00 & 4.1583 & 208.00 & 4.1613 & 304.00 & 4.1846 & & \\
\hline 114.00 & 4.1563 & 210.00 & 4.1604 & 306.00 & 4.1838 & & \\
\hline 116.00 & 4.1546 & 212.00 & 4.1600 & 308.00 & 4.1825 & & \\
\hline 118.00 & 4.1533 & 214.00 & 4.1604 & 310.00 & 4.1821 & & \\
\hline
\end{tabular}


Table C2. Thermo-Gravimetric Analysis Data

for $K$ Basin Fuel Sample Run $6 r$ at $200{ }^{\circ} \mathrm{C}$.

RUN NUMBER 6r AT $200 \mathrm{C}$

\begin{tabular}{|c|c|c|c|c|c|c|c|}
\hline $\begin{array}{c}\text { TIME } \\
\text { (minutes) }\end{array}$ & $\begin{array}{c}\text { WT GAIN } \\
(\mathrm{mg})\end{array}$ & $\begin{array}{c}\text { TiME } \\
\text { (minutes) }\end{array}$ & $\begin{array}{l}\text { WT GAIN } \\
\quad(\mathrm{mg})\end{array}$ & $\begin{array}{c}\text { TIME } \\
\text { (minutes) }\end{array}$ & $\begin{array}{c}\text { WT GAIN } \\
\text { (mg) }\end{array}$ & $\begin{array}{c}\text { TIME } \\
\text { (minutes) }\end{array}$ & $\begin{array}{c}\text { WT GAIN } \\
\text { (mg) }\end{array}$ \\
\hline 24.00 & 0.2079 & 120.00 & 0.2346 & 216.00 & 1.1138 & 312.00 & $\frac{1.29}{1.2925}$ \\
\hline 26.00 & 0.2113 & 122.00 & 0.2279 & 218.00 & 1.1188 & 314.00 & 1.3075 \\
\hline 28.00 & 0.2079 & 124.00 & 0.2333 & 220.00 & 1.1229 & 316.00 & 1.3450 \\
\hline 30.00 & 0.1983 & 126.00 & 0.2400 & 222.00 & 1.1233 & 318.00 & 1.4021 \\
\hline 32.00 & 0.1842 & 128.00 & 0.2400 & 224.00 & 1.1254 & 320.00 & 1.4604 \\
\hline 34.00 & 0.1833 & 130.00 & 0.2408 & 226.00 & 1.1258 & 322.00 & 1.4796 \\
\hline 36.00 & 0.1942 & 132.00 & 0.4567 & 228.00 & 1.1263 & 324.00 & 1.4788 \\
\hline 38.00 & 0.1950 & 134.00 & 0.5254 & 230.00 & 1.1283 & 326.00 & 1.4658 \\
\hline 40.00 & 0.1942 & 136.00 & 0.7479 & 232.00 & 1.1313 & 328.00 & 1.4796 \\
\hline 42.00 & 0.1992 & 138.00 & 0.8092 & 234.00 & 1.1342 & 330.00 & 1.5038 \\
\hline 44.00 & 0.2208 & 140.00 & 1.0025 & 236.00 & 1.1375 & 332.00 & 1.5267 \\
\hline 46.00 & 0.2363 & 142.00 & 1.0350 & 238.00 & 1.1421 & 334.00 & 1.5375 \\
\hline 48.00 & 0.2508 & 144.00 & 1.0158 & 240.00 & 1.1408 & 336.00 & 1.5154 \\
\hline 50.00 & 0.2458 & 146.00 & 1.0213 & 242.00 & 1.1325 & 338.00 & 1.4967 \\
\hline 52.00 & 0.2329 & 148.00 & 1.0192 & 244.00 & 1.1254 & 340.00 & 1.4992 \\
\hline 54.00 & 0.2158 & 150.00 & 1.0163 & 246.00 & 1.1196 & 342.00 & 1.5371 \\
\hline 56.00 & 0.2154 & 152.00 & 1.0142 & 248.00 & 1.1142 & 344.00 & 1.5688 \\
\hline 58.00 & 0.2250 & 154.00 & 1.0288 & 250.00 & 1.1050 & 346.00 & 1.5846 \\
\hline 60.00 & 0.2304 & 156.00 & 1.0467 & 252.00 & 1.0996 & 348.00 & 1.5663 \\
\hline 62.00 & 0.2392 & 158.00 & 1.0621 & 254.00 & 1.0963 & 350.00 & 1.5358 \\
\hline 64.00 & 0.2388 & 160.00 & 1.0683 & 256.00 & 1.0983 & 352.00 & 1.5192 \\
\hline 66.00 & 0.2313 & 162.00 & 1.0688 & 258.00 & 1.1042 & 354.00 & 1.5200 \\
\hline 68.00 & 0.2229 & 164.00 & 1.0717 & 260.00 & 1.1092 & 356.00 & 1.5246 \\
\hline 70.00 & 0.2275 & 166.00 & 1.0788 & 262.00 & 1.1146 & 358.00 & 1.5208 \\
\hline 72.00 & 0.2429 & 168.00 & 1.0871 & 264.00 & 1.1179 & 360.00 & 1.5217 \\
\hline 74.00 & 0.2529 & 170.00 & 1.0896 & 266.00 & 1.1250 & 362.00 & 1.5371 \\
\hline 76.00 & 0.2550 & 172.00 & 1.0863 & 268.00 & 1.1350 & 364.00 & 1.5513 \\
\hline 78.00 & 0.2542 & 174.00 & 1.0879 & 270.00 & 1.1408 & 366.00 & 1.5879 \\
\hline 80.00 & 0.2567 & 176.00 & 1.0921 & 272.00 & 1.1446 & 368.00 & 1.6446 \\
\hline 82.00 & 0.2596 & 178.00 & 1.0983 & 274.00 & 1.1442 & 370.00 & 1.7021 \\
\hline 84.00 & 0.2654 & 180.00 & 1.0988 & 276.00 & 1.1483 & 372.00 & 1.7325 \\
\hline 86.00 & 0.2654 & 182.00 & 1.0938 & 278.00 & 1.1525 & 374.00 & 1.7242 \\
\hline 88.00 & 0.2663 & 184.00 & 1.0958 & 280.00 & 1.1529 & 376.00 & 1.7208 \\
\hline 90.00 & 0.2675 & 186.00 & 1.0958 & 282.00 & 1.1458 & 378.00 & 1.7258 \\
\hline 92.00 & 0.2688 & 188.00 & 1.0983 & 284.00 & 1.1496 & 380.00 & 1.7375 \\
\hline 94.00 & 0.2700 & 190.00 & 1.0963 & 286.00 & 1.1513 & & \\
\hline 96.00 & 0.2679 & 192.00 & 1.0988 & 288.00 & 1.1704 & & \\
\hline 98.00 & 0.2738 & 194.00 & 1.1033 & 290.00 & 1.1604 & & \\
\hline 100.00 & 0.2833 & 196.00 & 1.1033 & 292.00 & 1.1654 & & \\
\hline 102.00 & 0.2921 & 198.00 & 1.1013 & 294.00 & 1.1779 & & \\
\hline 104.00 & 0.2921 & 200.00 & 1.0975 & 296.00 & 1.2092 & & \\
\hline 106.00 & 0.2821 & 202.00 & 1.1038 & 298.00 & 1.2379 & & \\
\hline 108.00 & 0.2638 & 204.00 & 1.1063 & 300.00 & 1.2346 & & \\
\hline 110.00 & 0.2558 & 206.00 & 1.1083 & 302.00 & 1.2363 & & \\
\hline 112.00 & 0.2517 & 208.00 & 1.1000 & 304.00 & 1.2421 & & \\
\hline 114.00 & 0.2604 & 210.00 & 1.0996 & 306.00 & 1.2663 & & \\
\hline 116.00 & 0.2567 & 212.00 & 1.1021 & 308.00 & 1.2879 & & \\
\hline 118.00 & 0.2483 & 214.00 & 1.1079 & 310.00 & 1.2933 & & \\
\hline
\end{tabular}


Table C3. Thermo-Gravimetric Analysis Data

for $\mathrm{K}$ Basin Fuel Sample Run 7 at $300{ }^{\circ} \mathrm{C}$.

\begin{tabular}{|c|c|c|c|c|c|c|c|}
\hline \multicolumn{8}{|c|}{ TGA DATA FOR RUN 7 AT $300 \mathrm{C}$} \\
\hline TIME & Wt Change & TIME & Wt Change & TIME & Wt Change & TIME & guver \\
\hline (minutes) & $(\mathrm{mg})$ & (minutes) & $(\mathrm{mg})$ & (minutes) & (mg) & (minutes) & (mg) \\
\hline 24.00 & 1.863 & 120.00 & -4.212 & 216.00 & 6.750 & 312.00 & 8.087 \\
\hline 26.00 & 0.458 & 122.00 & -4.329 & 218.00 & 8.121 & 314.00 & 8.783 \\
\hline 28.00 & -0.783 & 124.00 & -4.462 & 220.00 & 9.383 & 316.00 & 9.212 \\
\hline 30.00 & -1.400 & 126.00 & -4.496 & 222.00 & 9.392 & 318.00 & 6.758 \\
\hline 32.00 & -1.646 & 128.00 & -4.633 & 224.00 & 8.167 & 320.00 & 2.750 \\
\hline 34.00 & -1.854 & 130.00 & -4.563 & 226.00 & 6.704 & 322.00 & -1.754 \\
\hline 36.00 & -1.904 & 132.00 & -4.488 & 228.00 & 5.642 & 324.00 & -3.713 \\
\hline 38.00 & -2.050 & 134.00 & -4.458 & 230.00 & 5.738 & 326.00 & -4.308 \\
\hline 40.00 & -2.125 & 136.00 & -4.508 & 232.00 & 5.954 & 328.00 & -3.967 \\
\hline 42.00 & -2.088 & 138.00 & -4.488 & 234.00 & 6.125 & 330.00 & -1.217 \\
\hline 44.00 & -2.013 & 140.00 & -3.063 & 236.00 & 6.488 & 332.00 & 2.342 \\
\hline 46.00 & -1.888 & 142.00 & -1.683 & 238.00 & 7.063 & 334.00 & 4.667 \\
\hline 48.00 & -1.788 & 144.00 & -0.333 & 240.00 & 7.654 & 336.00 & 4.196 \\
\hline 50.00 & -1.696 & 146.00 & -0.013 & 242.00 & 7.783 & 338.00 & 2.942 \\
\hline 52.00 & -1.679 & 148.00 & 0.683 & 244.00 & 7.733 & 340.00 & 2.283 \\
\hline 54.00 & -1.758 & 150.00 & 1.554 & 246.00 & 7.517 & 342.00 & 2.500 \\
\hline 56.00 & -1.958 & 152.00 & 2.558 & 248.00 & 7.604 & 344.00 & 3.408 \\
\hline 58.00 & -2.154 & 154.00 & 3.296 & 250.00 & 7.538 & 346.00 & 4.563 \\
\hline 60.00 & -2.317 & 156.00 & 3.767 & 252.00 & 7.600 & 348.00 & 5.283 \\
\hline 62.00 & -2.350 & 158.00 & 3.921 & 254.00 & 7.533 & 350.00 & 4.450 \\
\hline 64.00 & -2.413 & 160.00 & 3.754 & 256.00 & 7.713 & 352.00 & 3.467 \\
\hline 66.00 & -2.333 & 162.00 & 3.721 & 258.00 & 7.950 & 354.00 & 3.079 \\
\hline 68.00 & -2.246 & 164.00 & 3.638 & 260.00 & 8.250 & 356.00 & 3.829 \\
\hline 70.00 & -2.208 & 166.00 & 3.813 & 262.00 & 8.400 & 358.00 & 4.958 \\
\hline 72.00 & -2.263 & 168.00 & 3.950 & 264.00 & 8.888 & 360.00 & 5.663 \\
\hline 74.00 & -2.338 & 170.00 & 4.021 & 266.00 & 9.696 & 362.00 & 5.988 \\
\hline 76.00 & -2.417 & 172.00 & 4.000 & 268.00 & 10.617 & 364.00 & 3.121 \\
\hline 78.00 & -2.533 & 174.00 & 4.017 & 270.00 & 11.146 & 366.00 & -1.917 \\
\hline 80.00 & -2.700 & 176.00 & 4.125 & 272.00 & 11.300 & 368.00 & -6.767 \\
\hline 82.00 & -2.742 & 178.00 & 4.317 & 274.00 & 11.725 & 370.00 & -8.788 \\
\hline 84.00 & -2.813 & 180.00 & 4.479 & 276.00 & 12.158 & 372.00 & -9.788 \\
\hline 86.00 & -2.858 & 182.00 & 4.608 & 278.00 & 12.796 & 374.00 & -11.150 \\
\hline 88.00 & -2.929 & 184.00 & 4.633 & 280.00 & 13.167 & 376.00 & -12.325 \\
\hline 90.00 & -2.917 & 186.00 & 4.671 & 282.00 & 13.629 & 378.00 & -12.729 \\
\hline 92.00 & -2.888 & 188.00 & 4.775 & 284.00 & 13.742 & 380.00 & -12.694 \\
\hline 94.00 & -2.829 & 190.00 & 5.054 & 286.00 & 13.433 & & \\
\hline 96.00 & -2.954 & 192.00 & 5.200 & 288.00 & 12.183 & & \\
\hline 98.00 & -3.163 & 194.00 & 5.317 & 290.00 & 11.000 & & \\
\hline 100.00 & -3.558 & 196.00 & 5.304 & 292.00 & 10.025 & & \\
\hline 102.00 & -3.796 & 198.00 & 5.413 & 294.00 & 8.871 & & \\
\hline 104.00 & -3.967 & 200.00 & 5.483 & 296.00 & 7.638 & & \\
\hline 106.00 & -3.858 & 202.00 & 5.500 & 298.00 & 6.633 & & \\
\hline 108.00 & -3.954 & 204.00 & 5.504 & 300.00 & 6.796 & & \\
\hline 110.00 & -4.012 & 206.00 & 5.475 & 302.00 & 6.917 & & \\
\hline 112.00 & -4.083 & 208.00 & 5.504 & 304.00 & 6.712 & & \\
\hline 114.00 & -4.058 & 210.00 & 5.462 & 306.00 & 6.492 & & \\
\hline 116.00 & -4.004 & 212.00 & 5.446 & 308.00 & 6.613 & & \\
\hline 118.00 & -4.096 & 214.00 & 5.604 & 310.00 & 7.258 & & \\
\hline
\end{tabular}


Table C4. Thermo-Gravimetric Analysis Data for $\mathrm{K}$ Basin Fuel Sample Run 8 at $400{ }^{\circ} \mathrm{C}$.

\begin{tabular}{|c|c|c|c|c|c|c|c|}
\hline \multicolumn{8}{|c|}{ TGA DATA FOR RUN NUMBER 8 AT 400 C } \\
\hline $\begin{array}{c}\text { TIME } \\
\text { (minutes) }\end{array}$ & $\begin{array}{c}\text { Wt Gain } \\
\text { (mg) }\end{array}$ & $\begin{array}{c}\text { TIME } \\
\text { (minutes) }\end{array}$ & $\begin{array}{c}\text { Wt Gain } \\
\text { (mg) }\end{array}$ & $\begin{array}{c}\text { TIME } \\
\text { (minutes) }\end{array}$ & $\begin{array}{c}\text { Wt Gain } \\
\text { (mg) }\end{array}$ & $\begin{array}{c}\text { TIME } \\
\text { (minutes) }\end{array}$ & $\begin{array}{c}\text { Wt Gain } \\
(\mathrm{mg})\end{array}$ \\
\hline 50.00 & 0.8129 & 146.00 & 6.6567 & 242.00 & 7.3408 & 338.00 & 8.6121 \\
\hline 52.00 & 0.8496 & 148.00 & 6.7042 & 244.00 & 7.3463 & 340.00 & 9.3458 \\
\hline 54.00 & 0.8879 & 150.00 & 6.7679 & 246.00 & 7.3708 & 342.00 & 10.8520 \\
\hline 56.00 & 0.9921 & 152.00 & 6.8033 & 248.00 & 7.4167 & 344.00 & 13.1580 \\
\hline 58.00 & 1.1892 & 154.00 & 6.8125 & 250.00 & 7.4792 & 346.00 & 15.3580 \\
\hline 60.00 & 1.4071 & 156.00 & 6.8158 & 252.00 & 7.5408 & 348.00 & 16.9720 \\
\hline 62.00 & 1.5633 & 158.00 & 6.8296 & 254.00 & 7.5892 & 350.00 & 17.8890 \\
\hline 64.00 & 1.6521 & 160.00 & 6.8542 & 256.00 & 7.6250 & 352.00 & 19.0100 \\
\hline 66.00 & 1.9517 & 162.00 & 6.8708 & 258.00 & 7.6442 & 354.00 & 20.9110 \\
\hline 68.00 & 2.5521 & 164.00 & 6.8808 & 260.00 & 7.6550 & 356.00 & 22.4370 \\
\hline 70.00 & 3.1746 & 166.00 & 6.8858 & 262.00 & 7.6750 & 358.00 & 23.4850 \\
\hline 72.00 & 3.5542 & 168.00 & 6.8896 & 264.00 & 7.6921 & 360.00 & 23.4850 \\
\hline 74.00 & 3.6433 & 170.00 & 6.9029 & 266.00 & 7.6933 & 362.00 & 23.4850 \\
\hline 76.00 & 3.8950 & 172.00 & 6.9208 & 268.00 & 7.7050 & 364.00 & 23.4850 \\
\hline 78.00 & 4.2763 & 174.00 & 6.9450 & 270.00 & 7.7300 & 366.00 & 23.4850 \\
\hline 80.00 & 4.6517 & 176.00 & 6.9621 & 272.00 & 7.7883 & 368.00 & 23.4850 \\
\hline 82.00 & 4.8150 & 178.00 & 6.9733 & 274.00 & 7.8450 & 370.00 & 23.4850 \\
\hline 84.00 & 4.8450 & 180.00 & 6.97 .75 & 276.00 & 7.8888 & 372.00 & 23.4850 \\
\hline 86.00 & 4.8813 & 182.00 & 6.9842 & 278.00 & 7.9104 & 374.00 & 23.4850 \\
\hline 88.00 & 4.9425 & 184.00 & 6.9958 & 280.00 & 7.9071 & 376.00 & 23.4850 \\
\hline 90.00 & 5.0304 & 186.00 & 7.0125 & 282.00 & 7.8792 & 378.00 & 23.4850 \\
\hline 92.00 & 5.1021 & 188.00 & 7.0283 & 284.00 & 7.8463 & 380.00 & 23.4850 \\
\hline 94.00 & 5.1557 & 190.00 & 7.0429 & 286.00 & 7.8300 & 382.00 & 23.4850 \\
\hline 96.00 & 5.2125 & 192.00 & 7.0608 & 288.00 & 7.8404 & 384.00 & 23.4850 \\
\hline 98.00 & 5.3150 & 194.00 & 7.0900 & 290.00 & 7.8604 & 386.00 & 23.4850 \\
\hline 100.00 & 5.4733 & 196.00 & 7.1225 & 292.00 & 7.8792 & & \\
\hline 102.00 & 5.6342 & 198.00 & 7.1475 & 294.00 & 7.9000 & & \\
\hline 104.00 & 5.7563 & 200.00 & 7.1638 & 296.00 & 7.9246 & & \\
\hline 106.00 & 5.8254 & 202.00 & 7.1771 & 298.00 & 7.9225 & & \\
\hline 108.00 & 5.8704 & 204.00 & 7.1871 & 300.00 & 7.9304 & & \\
\hline 110.00 & 5.9083 & 206.00 & 7.1971 & 302.00 & 7.9458 & & \\
\hline 112.00 & 5.9283 & 208.00 & 7.2071 & 304.00 & 7.9767 & & \\
\hline 114.00 & 5.9488 & 210.00 & 7.2483 & 306.00 & 7.9946 & & \\
\hline 116.00 & 5.9679 & 2.12 .00 & 7.2608 & 308.00 & 8.0038 & & \\
\hline 118.00 & 6.0025 & 214.00 & 7.2700 & 310.00 & 8.0192 & & \\
\hline 120.00 & 6.0375 & 216.00 & 7.2562 & 312.00 & 8.0279 & & \\
\hline 122.00 & 6.0621 & 218.00 & 7.2825 & 314.00 & 8.0325 & & \\
\hline 124.00 & 6.0871 & 220.00 & 7.3154 & 316.00 & 8.0438 & & \\
\hline 126.00 & 6.1183 & 222.00 & 7.3288 & 318.00 & 8.0604 & & \\
\hline 128.00 & 8.1938 & 224.00 & 7.3183 & 320.00 & 8.0875 & & \\
\hline 130.00 & 6.3175 & 226.00 & 7.2942 & 322.00 & 8.1504 & & \\
\hline 132.00 & 6.4200 & 228.00 & 7.2846 & 324.00 & 8.2333 & & \\
\hline 134.00 & 6.4821 & 230.00 & 7.2883 & 326.00 & 8.2754 & & \\
\hline 136.00 & 6.4942 & 232.00 & 7.3042 & 328.00 & 8.2317 & & \\
\hline 138.00 & 6.5213 & 234.00 & 7.3171 & 330.00 & 8.1650 & & \\
\hline 140.00 & 6.5567 & 236.00 & 7.3279 & 332.00 & 8.1304 & & \\
\hline 142.00 & 6.5933 & 238.00 & 7.3288 & 334.00 & 8.1479 & & \\
\hline 144.00 & 6.6208 & 240.00 & 7.3337 & 336.00 & 8.2458 & & \\
\hline
\end{tabular}


Table C5. Thermo-Gravimetric Analysis Data

for $K$ Basin Fuel Sample Run 18 at $144{ }^{\circ} \mathrm{C}$.

\begin{tabular}{|c|c|c|c|c|c|c|c|}
\hline \multicolumn{8}{|c|}{ RUN NUMBER 18 AT $144 \mathrm{C}$} \\
\hline $\begin{array}{c}\text { TIME } \\
\text { (minutes) }\end{array}$ & $\begin{array}{c}\text { Wt Gain } \\
\text { (mg) }\end{array}$ & $\begin{array}{c}\text { TIME } \\
\text { (minutes) }\end{array}$ & $\begin{array}{c}\text { Wt Gain } \\
\text { (mg) }\end{array}$ & $\begin{array}{c}\text { TIME } \\
\text { (minutes) }\end{array}$ & $\begin{array}{c}\text { Wt Gain } \\
(\mathrm{mg})\end{array}$ & $\begin{array}{c}\text { TIME } \\
\text { (minutes) }\end{array}$ & $\begin{array}{c}\text { Wt Gain } \\
\text { (mg) }\end{array}$ \\
\hline 25.00 & -0.1017 & 105.00 & -0.2358 & 185.00 & 3.8117 & 265.00 & 5.6308 \\
\hline 26.67 & -0.1013 & 106.67 & -0.2338 & 186.67 & 3.5938 & 266.67 & 5.6346 \\
\hline 28.33 & -0.1096 & 108.33 & -0.2417 & 188.33 & 3.4892 & 268.33 & 5.5708 \\
\hline 30.00 & -0.1054 & 110.00 & -0.2504 & 190.00 & 3.3417 & 270.00 & 5.6025 \\
\hline 31.67 & -0.1042 & 111.67 & -0.2608 & 191.67 & 3.0204 & 271.67 & 5.5800 \\
\hline 33.33 & -0.0975 & 113.33 & -0.2667 & 193.33 & 3.0075 & 273.33 & 5.6017 \\
\hline 35.00 & -0.0871 & 115.00 & -0.2704 & 195.00 & 3.3058 & 275.00 & 5.5983 \\
\hline 36.67 & -0.0767 & 116.67 & -0.2758 & 196.67 & 3.6008 & 276.67 & 5.6054 \\
\hline 38.33 & -0.0712 & 118.33 & -0.2808 & 198.33 & 3.7492 & 278.33 & 5.6225 \\
\hline 40.00 & -0.0746 & 120.00 & -0.2875 & 200.00 & 3.7892 & 280.00 & 5.6267 \\
\hline 41.67 & -0.0867 & 121.67 & -0.2883 & 201.67 & 3.8958 & 281.67 & 5.6442 \\
\hline 43.33 & -0.1050 & 123.33 & -0.2913 & 203.33 & 4.0600 & 283.33 & 5.6450 \\
\hline 45.00 & -0.1208 & 125.00 & -0.2917 & 205.00 & 4.2904 & 285.00 & 5.6633 \\
\hline 46.67 & -0.1304 & 126.67 & -0.2888 & 206.67 & 4.5900 & 286.67 & 5.6746 \\
\hline 48.33 & -0.1379 & 128.33 & -0.2838 & 208.33 & 4.8600 & 288.33 & 5.6596 \\
\hline 50.00 & -0.1471 & 130.00 & -0.2767 & 210.00 & 5.1233 & 290.00 & 5.6708 \\
\hline 51.67 & -0.1575 & 131.67 & -0.2746 & 211.67 & 5.2700 & 291.67 & 5.6592 \\
\hline 53.33 & -0.1717 & 133.33 & -0.2738 & 213.33 & 5.3425 & 293.33 & 5.6771 \\
\hline 55.00 & -0.1842 & 135.00 & -0.2763 & 215.00 & 5.3517 & 295.00 & 5.6775 \\
\hline 56.67 & -0.1925 & 136.67 & -0.2792 & 216.67 & 5.3538 & 296.67 & 5.7092 \\
\hline 58.33 & -0.1892 & 138.33 & -0.2783 & 218.33 & 5.3625 & 298.33 & 5.6996 \\
\hline 60.00 & -0.1850 & 140.00 & -0.2792 & 220.00 & 5.3779 & 300.00 & 5.6833 \\
\hline 61.67 & -0.1783 & 141.67 & -0.2788 & 221.67 & 5.4129 & 301.67 & 5.6767 \\
\hline 63.33 & -0.1767 & 143.33 & -0.2708 & 223.33 & 5.4333 & 303.33 & 5.6621 \\
\hline 65.00 & -0.1725 & 145.00 & -0.2667 & 225.00 & 5.4438 & 305.00 & 5.6650 \\
\hline 66.67 & -0.1738 & 146.67 & -0.2583 & 226.67 & 5.4288 & 306.67 & 5.6463 \\
\hline 68.33 & -0.1775 & 148.33 & -0.2567 & 228.33 & 5.4529 & 308.33 & 5.6804 \\
\hline 70.00 & -0.1833 & 150.00 & -0.2504 & 230.00 & 5.4825 & 310.00 & 5.6894 \\
\hline 71.67 & -0.1846 & 151.67 & -0.2442 & 231.67 & 5.5388 & & \\
\hline 73.33 & -0.1854 & 153.33 & -0.2358 & 233.33 & 5.5650 & & \\
\hline 75.00 & -0.1908 & 155.00 & 1.7167 & 235.00 & 5.5858 & & \\
\hline 76.67 & -0.1971 & 156.67 & 4.0613 & 236.67 & 5.5642 & & \\
\hline 78.33 & -0.2025 & 158.33 & 6.3188 & 238.33 & 5.5408 & & \\
\hline 80.00 & -0.2042 & 160.00 & 6.3696 & 240.00 & 5.5175 & & \\
\hline 81.67 & -0.2083 & 161.67 & 5.9950 & 241.67 & 5.5554 & & \\
\hline 83.33 & -0.2083 & 163.33 & 5.7050 & 243.33 & 5.5963 & & \\
\hline 85.00 & -0.2088 & 165.00 & 5.6688 & 245.00 & 5.5796 & & \\
\hline 86.67 & -0.2067 & 166.67 & 5.6654 & 246.67 & 5.5479 & & \\
\hline 88.33 & -0.2108 & 168.33 & 5.6450 & 248.33 & 5.4992 & & \\
\hline 90.00 & -0.2179 & 170.00 & 5.6292 & 250.00 & 5.5196 & & \\
\hline 91.67 & -0.2308 & 171.67 & 5.6083 & 251.67 & 5.5192 & & \\
\hline 93.33 & -0.2442 & 173.33 & 5.5946 & 253.33 & 5.5454 & & \\
\hline 95.00 & -0.2563 & 175.00 & 5.5850 & 255.00 & 5.5592 & & \\
\hline 96.67 & -0.2617 & 176.67 & 5.5771 & 256.67 & 5.5988 & & \\
\hline 98.33 & -0.2579 & 178.33 & 5.5671 & 258.33 & 5.6100 & & \\
\hline 100.00 & -0.2517 & 180.00 & 5.4946 & 260.00 & 5.5704 & & \\
\hline 101.67 & -0.2458 & 181.67 & 5.1108 & 261.67 & 5.5654 & & \\
\hline 103.33 & -0.2404 & 183.33 & 4.5004 & 263.33 & 5.6142 & & \\
\hline
\end{tabular}


Table C6. Thermo-Gravimetric Analysis Data for $\mathrm{K}$ Basin Fuel Sample Run $18 \mathrm{~b}$ at $150{ }^{\circ} \mathrm{C}$. (Page 1 of 3 )

\begin{tabular}{|c|c|c|c|c|c|c|c|}
\hline \multicolumn{8}{|c|}{ RUN NUMBER $18 b$ AT $150 \mathrm{C}$} \\
\hline TIME & Wt Gain & TIME & Wt Gain & TIME & Wt Gain & TIME & Wt Gain \\
\hline (minutes) & $(\mathrm{mg})$ & (minutes) & (mg) & (minutes) & (mg) & (minutes) & $(\mathrm{mg})$ \\
\hline$\overline{|c| 0.0}$ & -0.272 & 35.0 & -0.095 & 60.0 & 0.152 & 85.0 & 0.173 \\
\hline 10.5 & -0.273 & 35.5 & -0.063 & 60.5 & 0.173 & 85.5 & 0.182 \\
\hline 11.0 & -0.264 & 36.0 & -0.043 & 61.0 & 0.163 & 86.0 & 0.179 \\
\hline 11.5 & -0.267 & 36.5 & -0.087 & 61.5 & 0.147 & 86.5 & 0.176 \\
\hline 12.0 & -0.236 & 37.0 & -0.052 & 62.0 & 0.163 & 87.0 & 0.197 \\
\hline 12.5 & -0.234 & 37.5 & -0.059 & 62.5 & 0.160 & 87.5 & 0.175 \\
\hline 13.0 & -0.241 & 38.0 & -0.078 & 63.0 & 0.148 & 88.0 & 0.187 \\
\hline 13.5 & -0.246 & 38.5 & -0.050 & 63.5 & 0.170 & 88.5 & 0.185 \\
\hline 14.0 & -0.232 & 39.0 & -0.061 & 64.0 & 0.166 & 89.0 & 0.167 \\
\hline 14.5 & -0.232 & 39.5 & -0.086 & 64.5 & 0.156 & 89.5 & 0.203 \\
\hline 15.0 & -0.195 & 40.0 & -0.029 & 65.0 & 0.165 & 90.0 & 0.200 \\
\hline 15.5 & -0.190 & 40.5 & -0.039 & 65.5 & 0.159 & 90.5 & 0.175 \\
\hline 16.0 & -0.217 & 41.0 & -0.073 & 66.0 & 0.152 & 91.0 & 0.204 \\
\hline 16.5 & -0.180 & 41.5 & -0.062 & 66.5 & 0.163 & 91.5 & 0.195 \\
\hline 17.0 & -0.186 & 42.0 & -0.034 & 67.0 & 0.158 & 92.0 & 0.183 \\
\hline 17.5 & -0.210 & 42.5 & 0.215 & 67.5 & 0.175 & 92.5 & 0.203 \\
\hline 18.0 & -0.065 & 43.0 & 0.207 & 68.0 & 0.160 & 93.0 & 0.186 \\
\hline 18.5 & -0.144 & 43.5 & 0.213 & 68.5 & 0.171 & 93.5 & 0.168 \\
\hline 19.0 & -0.184 & 44.0 & 0.174 & 69.0 & 0.180 & 94.0 & 0.222 \\
\hline 19.5 & -0.153 & 44.5 & 0.177 & 69.5 & 0.161 & 94.5 & 0.207 \\
\hline 20.0 & -0.140 & 45.0 & 0.181 & 70.0 & 0.179 & 95.0 & 0.198 \\
\hline 20.5 & -0.171 & 45.5 & 0.169 & 70.5 & 0.173 & 95.5 & 0.220 \\
\hline 21.0 & -0.148 & 46.0 & 0.166 & 71.0 & 0.158 & 96.0 & 0.196 \\
\hline 21.5 & -0.136 & 46.5 & 0.170 & 71.5 & 0.163 & 96.5 & 0.203 \\
\hline 22.0 & -0.160 & 47.0 & 0.176 & 72.0 & 0.187 & 97.0 & 0.210 \\
\hline 22.5 & -0.1413 & 47.5 & 0.161 & 72.5 & 0.152 & 97.5 & 0.195 \\
\hline 23.0 & -0.140 & 48.0 & 63 & 73.0 & 0.185 & 98.0 & 0.203 \\
\hline 23.5 & -0.153 & 48.5 & 75 & 73.5 & 0.175 & 98.5 & 0.208 \\
\hline 24.0 & -0.103 & 49.0 & 0.154 & 74.0 & 0.156 & 99.0 & 0.190 \\
\hline 24.5 & -0.118 & 49.5 & 0.170 & 74.5 & 0.195 & 99.5 & 0.211 \\
\hline 25.0 & -0.145 & 50.0 & 0.167 & 75.0 & 0.170 & 100.0 & 0.212 \\
\hline 25.5 & -0.131 & 50.5 & 0.146 & 75.5 & 0.162 & 100.5 & 0.200 \\
\hline 26.0 & -0.132 & 51.0 & 0.153 & 76.0 & 0.175 & 101.0 & 0.227 \\
\hline 26.5 & -0.144 & 51.5 & 185 & 76.5 & 0.164 & 101.5 & 0.209 \\
\hline 27.0 & -0.124 & 52.0 & 0.152 & 77.0 & 0.173 & 102.0 & 0.203 \\
\hline 27.5 & -0.121 & 52.5 & 155 & 77.5 & 0.168 & 102.5 & 0.220 \\
\hline 28.0 & -0.125 & 53.0 & $0.176^{8}$ & 78.0 & 0.165 & 103.0 & 0.202 \\
\hline 28.5 & -0.128 & 53.5 & 0.144 & 78.5 & 0.175 & 103.5 & 0.210 \\
\hline 29.0 & -0.110 & 54.0 & 0.142 & 79.0 & 0.159 & 104.0 & 0.215 \\
\hline 29.5 & -0.114 & 54.5 & 0.195 & 79.5 & 0.160 & 104.5 & 0.209 \\
\hline 30.0 & -0.131 & 55.0 & 0.153 & 80.0 & 0.167 & 105.0 & 0.221 \\
\hline 30.5 & -0.121 & 55.5 & 0.155 & 80.5 & 0.160 & 105.5 & 0.220 \\
\hline 31.0 & -0.103 & 56.0 & 0.176 & 81.0 & 0.177 & 106.0 & 0.211 \\
\hline 31.5 & -0.111 & 56.5 & 0.158 & 81.5 & 0.176 & 106.5 & 0.215 \\
\hline 32.0 & -0.113 & 57.0 & 0.153 & 82.0 & 0.170 & 107.0 & 0.217 \\
\hline 32.5 & -0.085 & 57.5 & 0.168 & 82.5 & 0.173 & 107.5 & 0.213 \\
\hline 33.0 & -0.118 & 58.0 & 0.162 & 83.0 & 0.181 & 108.0 & 0.209 \\
\hline 33.5 & -0.124 & 58.5 & 0.146 & 83.5 & 0.171 & 108.5 & 0.203 \\
\hline 34.0 & -0.067 & 59.0 & 0.175 & 84.0 & 0.177 & 109.0 & 0.221 \\
\hline 34.5 & -0.098 & 59.5 & 0.165 & 84.5 & 0.159 & 109.5 & 0.205 \\
\hline
\end{tabular}


Table C6. Thermo-Gravimetric Analysis Data for $\mathrm{K}$ Basin Fuel Sample Run $18 \mathrm{~b}$ at $150^{\circ} \mathrm{C}$. (Page 2 of 3 )

\begin{tabular}{|c|c|c|c|c|c|c|c|}
\hline \multicolumn{8}{|c|}{ RUN NUMBER $18 \mathrm{~b}$ AT $150 \mathrm{C}$ (cont.) } \\
\hline TIME & Wt Gain & TIME & Wt Gain & TIME & Wt Gain & TIME & Wt Gain \\
\hline (minutes) & $(\mathrm{mg})$ & (minutes) & $(\mathrm{mg})$ & (minutes) & (mg) & (minutes) & (mg) \\
\hline 110.0 & 0.211 & 135.0 & 0.315 & 160.0 & 0.221 & 185.0 & 0.143 \\
\hline 110.5 & 0.228 & 135.5 & 0.309 & 160.5 & 0.190 & 185.5 & 0.139 \\
\hline 111.0 & 0.203 & 136.0 & 0.366 & 161.0 & 0.186 & 186.0 & 0.142 \\
\hline 111.5 & 0.220 & 136.5 & 0.294 & 161.5 & 0.195 & 186.5 & 0.147 \\
\hline 112.0 & 0.211 & 137.0 & 0.290 & 162.0 & 0.189 & 187.0 & 0.141 \\
\hline 112.5 & 0.212 & 137.5 & 0.325 & 162.5 & 0.178 & 187.5 & 0.195 \\
\hline 113.0 & 0.215 & 138.0 & 0.268 & 163.0 & 0.194 & 188.0 & 0.142 \\
\hline 113.5 & 0.218 & 138.5 & 0.299 & 163.5 & 0.179 & 188.5 & 0.134 \\
\hline 114.0 & 0.210 & 139.0 & 0.286 & 164.0 & 0.189 & 189.0 & 0.159 \\
\hline 114.5 & 0.229 & 139.5 & 0.254 & 164.5 & 0.189 & 189.5 & 0.140 \\
\hline 115.0 & $\overline{0.222}$ & 140.0 & 0.252 & 165.0 & 0.170 & 190.0 & 0.140 \\
\hline 115.5 & 0.206 & 140.5 & 0.270 & 165.5 & 0.190 & 190.5 & 0.158 \\
\hline 116.0 & 0.231 & 141.0 & 0.244 & 166.0 & 0.189 & 191.0 & 3.365 \\
\hline 116.5 & 0.215 & 141.5 & 0.288 & 166.5 & 0.157 & 191.5 & 1.027 \\
\hline 117.0 & 0.221 & 142.0 & 0.258 & 167.0 & 0.181 & 192.0 & 0.930 \\
\hline 117.5 & 0.216 & 142.5 & 0.230 & 167.5 & 0.174 & 192.5 & 0.932 \\
\hline 118.0 & 0.217 & 143.0 & 0.266 & 168.0 & 0.152 & 193.0 & 0.965 \\
\hline 118.5 & 0.207 & 143.5 & 0.232 & 168.5 & 0.174 & 193.5 & 0.913 \\
\hline 119.0 & 0.240 & 144.0 & 0.231 & 169.0 & 0.168 & 194.0 & 0.887 \\
\hline 119.5 & 0.221 & 144.5 & 0.251 & 169.5 & 0.163 & 194.5 & 0.855 \\
\hline 120.0 & 0.221 & 145.0 & 0.205 & 170.0 & 0.183 & 195.0 & 0.856 \\
\hline 120.5 & 0.240 & 145.5 & 0.225 & 170.5 & 0.165 & 195.5 & 0.849 \\
\hline 121.0 & 0.205 & 146.0 & 0.237 & 171.0 & 0.213 & 196.0 & 0.842 \\
\hline 121.5 & 0.219 & 146.5 & 0.212 & 171.5 & 0.189 & 196.5 & 0.805 \\
\hline 122.0 & 0.253 & 147.0 & 0.229 & 172.0 & 0.165 & 197.0 & 1.013 \\
\hline 122.5 & 0.213 & 147.5 & 0.243 & 172.5 & 0.169 & 197.5 & 0.848 \\
\hline 123.0 & 0.230 & 148.0 & 0.199 & 173.0 & 0.186 & 198.0 & 0.817 \\
\hline 123.5 & 0.236 & 148.5 & 0.235 & 173.5 & 0.173 & 198.5 & 0.835 \\
\hline 124.0 & 0.210 & 149.0 & 0.235 & 174.0 & 0.156 & 199.0 & 0.818 \\
\hline 124.5 & 0.229 & 149.5 & 0.197 & 174.5 & 0.172 & 199.5 & 0.846 \\
\hline 125.0 & 0.232 & 150.0 & 0.232 & 175.0 & 0.173 & 200.0 & 0.819 \\
\hline 125.5 & 0.217 & 150.5 & 0.199 & 175.5 & 0.149 & 200.5 & 1.118 \\
\hline 126.0 & 0.243 & 151.0 & 0.194 & 176.0 & 0.150 & 201.0 & 0.809 \\
\hline 126.5 & 0.204 & 151.5 & 0.235 & 176.5 & 0.158 & 201.5 & 0.818 \\
\hline 127.0 & 0.210 & 152.0 & 0.198 & 177.0 & 0.159 & 202.0 & 0.785 \\
\hline 127.5 & 0.251 & 152.5 & 0.214 & 177.5 & 0.146 & 202.5 & 0.765 \\
\hline 128.0 & 0.212 & 153.0 & 0.258 & 178.0 & 0.158 & 203.0 & 0.761 \\
\hline 128.5 & 0.224 & 153.5 & 0.184 & 178.5 & 0.141 & 203.5 & 0.773 \\
\hline 129.0 & 0.236 & 154.0 & 0.213 & 179.0 & 0.151 & 204.0 & 0.784 \\
\hline 129.5 & .215 & 154.5 & 0.217 & 179.5 & 0.150 & 204.5 & 0.744 \\
\hline 130.0 & 0.226 & 155.0 & 0.194 & 180.0 & 0.129 & 205.0 & 0.750 \\
\hline 130.5 & 0.220 & 155.5 & 0.223 & 180.5 & 0.165 & 205.5 & 0.753 \\
\hline 131.0 & 0.207 & 156.0 & 0.212 & 181.0 & 0.138 & 206.0 & 0.735 \\
\hline 131.5 & 0.259 & 156.5 & 0.191 & 181.5 & 0.136 & 206.5 & 0.725 \\
\hline 132.0 & 0.221 & 157.0 & 0.224 & 182.0 & 0.135 & 207.0 & 0.725 \\
\hline 132.5 & 0.218 & 157.5 & 0.208 & 182.5 & 0.133 & 207.5 & 0.729 \\
\hline 133.0 & 0.607 & 158.0 & 0.195 & 183.0 & 0.169 & 208.0 & 0.716 \\
\hline 133.5 & 0.512 & 158.5 & 0.223 & 183.5 & 0.158 & 208.5 & 0.711 \\
\hline 134.0 & 0.424 & 159.0 & 0.216 & 184.0 & 0.131 & 209.0 & 0.732 \\
\hline 134.5 & 0.395 & 159.5 & 0.181 & 184.5 & 0.144 & 209.5 & 0.685 \\
\hline
\end{tabular}


Table C6. Thermo-Gravimetric Analysis Data for $\mathrm{K}$ Basin Fuel Sample Run $18 \mathrm{~b}$ at $150{ }^{\circ} \mathrm{C}$. (Page 3 of 3 )

\begin{tabular}{|c|c|c|c|c|c|c|c|}
\hline \multicolumn{8}{|c|}{ RUN NUMBER $18 \mathrm{~b}$ AT $150 \mathrm{C}$ (cont.) } \\
\hline TIME & Wt Gain & TIME & Wt Gain & TIME & Wt Gain & TIME & Wt Gain \\
\hline (minutes) & (mg) & (minutes) & (mg) & (minutes) & $(\mathrm{mg})$ & (minutes) & (mg) \\
\hline 210.0 & 0.663 & 235.0 & 0.530 & 260.0 & 0.455 & 285.0 & 0.351 \\
\hline 210.5 & 0.682 & 235.5 & 0.540 & 260.5 & 0.447 & 285.5 & 0.313 \\
\hline 211.0 & 0.660 & 236.0 & 0.493 & 261.0 & 0.439 & 286.0 & 0.307 \\
\hline 211.5 & 0.661 & 236.5 & 0.509 & 261.5 & 0.456 & 286.5 & 0.312 \\
\hline 212.0 & 0.626 & 237.0 & 0.510 & 262.0 & 0.441 & 287.0 & 0.316 \\
\hline 212.5 & 0.641 & 237.5 & 0.507 & 262.5 & 0.455 & 287.5 & 0.300 \\
\hline 213.0 & 0.626 & 238.0 & 0.523 & 263.0 & 0.425 & 288.0 & 0.294 \\
\hline 213.5 & 0.619 & 238.5 & 0.513 & 263.5 & 0.430 & 288.5 & 0.294 \\
\hline 214.0 & 0.599 & 239.0 & 0.498 & 264.0 & 0.457 & 289.0 & 0.288 \\
\hline 214.5 & 0.626 & 239.5 & 0.504 & 264.5 & 0.437 & 289.5 & 0.257 \\
\hline 215.0 & 0.615 & 240.0 & 0.498 & 265.0 & 0.420 & 290.0 & 0.313 \\
\hline 215.5 & 0.619 & 240.5 & 0.504 & 265.5 & 0.431 & 290.5 & 0.279 \\
\hline 216.0 & 0.594 & 241.0 & 0.514 & 266.0 & 0.430 & 291.0 & 0.285 \\
\hline 216.5 & 0.612 & 241.5 & 0.505 & 266.5 & 0.402 & 291.5 & 0.300 \\
\hline 217.0 & 0.583 & 242.0 & 0.491 & 267.0 & 0.419 & 292.0 & 0.270 \\
\hline 217.5 & 0.605 & 242.5 & 0.502 & 267.5 & 0.421 & 292.5 & 0.273 \\
\hline 218.0 & 0.586 & 243.0 & 0.495 & 268.0 & 0.397 & 293.0 & 0.279 \\
\hline 218.5 & 0.607 & 243.5 & 0.501 & 268.5 & 0.413 & 293.5 & 0.271 \\
\hline 219.0 & 0.615 & 244.0 & 0.482 & 269.0 & 0.418 & 294.0 & 0.273 \\
\hline 219.5 & 0.596 & 244.5 & 0.490 & 269.5 & 0.410 & 294.5 & 0.288 \\
\hline 220.0 & 0.602 & 245.0 & 0.497 & 270.0 & 0.384 & 295.0 & 0.297 \\
\hline 220.5 & 0.601 & 245.5 & 0.469 & 270.5 & 0.396 & 295.5 & 0.282 \\
\hline 221.0 & 0.590 & 246.0 & 0.491 & 271.0 & 0.413 & 296.0 & 0.255 \\
\hline 221.5 & 0.605 & 246.5 & 0.482 & 271.5 & 0.395 & 296.5 & 0.283 \\
\hline 222.0 & 0.575 & 247.0 & 0.470 & 272.0 & 0.393 & 297.0 & 0.258 \\
\hline 222.5 & 0.557 & 247.5 & 0.460 & 272.5 & 0.376 & 297.5 & 0.267 \\
\hline 223.0 & 0.591 & 248.0 & 0.445 & 273.0 & 0.386 & 298.0 & 0.262 \\
\hline 223.5 & 0.563 & 248.5 & 0.477 & 273.5 & 0.355 & 298.5 & 0.257 \\
\hline 224.0 & 0.583 & 249.0 & 0.467 & 274.0 & 0.376 & 299.0 & 0.265 \\
\hline 224.5 & 0.578 & 249.5 & 0.502 & 274.5 & 0.377 & 299.5 & 0.246 \\
\hline 225.0 & 0.575 & 250.0 & 0.488 & 275.0 & 0.373 & 300.0 & 0.265 \\
\hline 225.5 & 0.583 & 250.5 & 0.465 & 275.5 & 0.378 & 300.5 & 0.247 \\
\hline 226.0 & 0.588 & 251.0 & 0.470 & 276.0 & 0.395 & 301.0 & 0.274 \\
\hline 226.5 & 0.567 & 251.5 & 0.467 & 276.5 & 0.349 & 301.5 & 0.247 \\
\hline 227.0 & 0.576 & 252.0 & 0.462 & 277.0 & 0.370 & 302.0 & 0.270 \\
\hline 227.5 & 0.574 & 252.5 & 0.446 & 277.5 & 0.355 & 302.5 & 0.243 \\
\hline 228.0 & 0.555 & 253.0 & 0.460 & 278.0 & 0.343 & 303.0 & 0.237 \\
\hline 228.5 & 0.567 & 253.5 & 0.434 & 278.5 & 0.316 & 303.5 & 0.238 \\
\hline 229.0 & 0.567 & 254.0 & 0.463 & 279.0 & 0.419 & 304.0 & 0.243 \\
\hline 229.5 & 0.545 & 254.5 & 0.459 & 279.5 & 0.353 & 304.5 & 0.270 \\
\hline 230.0 & 0.558 & 255.0 & 0.458 & 280.0 & 0.390 & 305.0 & 0.270 \\
\hline 230.5 & 0.548 & 255.5 & 0.434 & 280.5 & 0.370 & 305.5 & 0.254 \\
\hline 231.0 & 0.532 & 256.0 & 0.440 & 281.0 & 0.350 & 306.0 & 0.250 \\
\hline 231.5 & 0.561 & 256.5 & 0.434 & 281.5 & 0.375 & 306.5 & 0.234 \\
\hline 232.0 & 0.541 & 257.0 & 0.444 & 282.0 & 0.350 & 307.0 & 0.237 \\
\hline 232.5 & 0.553 & 257.5 & 0.437 & 282.5 & 0.369 & 307.5 & 0.255 \\
\hline 233.0 & 0.540 & 258.0 & 0.454 & 283.0 & 0.351 & 308.0 & 0.237 \\
\hline 233.5 & 0.515 & 258.5 & 0.446 & 283.5 & 0.334 & 308.5 & 0.257 \\
\hline 234.0 & 0.525 & 259.0 & 0.460 & 284.0 & 0.320 & 309.0 & 0.242 \\
\hline 234.5 & 0.547 & 259.5 & 0.460 & 284.5 & 0.355 & 309.5 & 0.259 \\
\hline
\end{tabular}


HNF-SD-SNF-CN-035, Rev. 0

Table C7. Thermo-Gravimetric Analysis Data

for $K$ Basin Fuel Sample Run $19 a$ at $200{ }^{\circ} \mathrm{C}$.

\begin{tabular}{|c|c|c|c|c|c|c|c|c|c|c|}
\hline \multicolumn{11}{|c|}{ RUN NUMBER 19a AT 200C } \\
\hline TIME & Wt Gain & & TIME & Wt Gain & & TIME & Wt Gain & & TIME & Wt Gain \\
\hline (minutes) & (mg) & & (minutes) & (mg) & & (minutes) & (mg) & & (minutes) & (mg) \\
\hline 25.00 & 1.0621 & & 103.33 & 3.7921 & & 181.67 & 5.7750 & & 260.00 & 7.4225 \\
\hline 26.67 & 1.1321 & & 105.00 & 3.8413 & & 183.33 & 5.8167 & & 261.67 & 7.4550 \\
\hline 28.33 & 1.2092 & & 106.67 & 3.8904 & & 185.00 & 5.8608 & & 263.33 & 7.4888 \\
\hline 30.00 & 1.2888 & & 108.33 & 3.9346 & & 186.67 & 5.9038 & & 265.00 & 7.5212 \\
\hline 31.67 & 1.3646 & & 110.00 & 3.9775 & & 188.33 & 5.9404 & & 266.67 & 7.5525 \\
\hline 33.33 & 1.4425 & & 111.67 & 4.0204 & & 190.00 & 5.9754 & & 268.33 & 7.5821 \\
\hline 35.00 & 1.5188 & & 113.33 & 4.0683 & & 191.67 & 6.0117 & & 270.00 & 7.6129 \\
\hline 36.67 & 1.5929 & & 115.00 & 4.1125 & & 193.33 & 6.0517 & & 271.67 & 7.6446 \\
\hline 38.33 & 1.6617 & & 116.67 & 4.1571 & & 195.00 & 6.0900 & & 273.33 & 7.6804 \\
\hline 40.00 & 1.7329 & & 118.33 & 4.1992 & & 196.67 & 6.1283 & & 275.00 & 7.7154 \\
\hline 41.67 & 1.8075 & & 120.00 & 4.2433 & & 198.33 & 6.1646 & & 276.67 & 7.7500 \\
\hline 43.33 & 1.8721 & & 121.67 & 4.2871 & & 200.00 & 6.2000 & & 278.33 & 7.7800 \\
\hline 45.00 & 1.9292 & & 123.33 & 4.3292 & & 201.67 & 6.2346 & & 280.00 & 7.8096 \\
\hline 46.67 & 1.9800 & & 125.00 & 4.3696 & & 203.33 & 6.2712 & & 281.67 & 7.8404 \\
\hline 48.33 & 2.0379 & & 126.67 & 4.4108 & & 205.00 & 6.3079 & & 283.33 & 7.8733 \\
\hline 50.00 & 2.0950 & & 128.33 & 4.4529 & & 206.67 & 6.3463 & & 285.00 & 7.9075 \\
\hline 51.67 & 2.1592 & & 130.00 & 4.4971 & & 208.33 & 6.3825 & & 286.67 & 7.9413 \\
\hline 53.33 & 2.2288 & & 131.67 & 4.5454 & & 210.00 & 6.4213 & & 288.33 & 7.9746 \\
\hline 55.00 & 2.2929 & & 133.33 & 4.5929 & & 211.67 & 6.4575 & & 290.00 & 8.0075 \\
\hline 56.67 & 2.3458 & & 135.00 & 4.6333 & & 213.33 & 6.4954 & & 291.67 & 8.0404 \\
\hline 58.33 & 2.3900 & & 136.67 & 4.6750 & & 215.00 & 6.5308 & & 293.33 & 8.0746 \\
\hline 60.00 & 2.4413 & & 138.33 & 4.7133 & & 216.67 & 6.5663 & & 295.00 & 8.1071 \\
\hline 61.67 & 2.5000 & & 140.00 & 4.7575 & & 218.33 & 6.6000 & & 296.67 & 8.1383 \\
\hline 63.33 & 2.5688 & & 141.67 & 4.7975 & & 220.00 & 6.6342 & & 298.33 & 8.1679 \\
\hline 65.00 & 2.6329 & & 143.33 & 4.8425 & & 221.67 & 6.6675 & & 300.00 & 8.1992 \\
\hline 66.67 & 2.6921 & & 145.00 & 4.8850 & & 223.33 & 6.7017 & & 301.67 & 8.2304 \\
\hline 68.33 & 2.7413 & & 146.67 & 4.9225 & & 225.00 & 6.7358 & & 303.33 & 8.2621 \\
\hline 70.00 & 2.7946 & & 148.33 & 4.9621 & & 226.67 & 6.7704 & & 305.00 & 8.2917 \\
\hline 71.67 & 2.8513 & & 150.00 & 5.0021 & & 228.33 & 6.8008 & & 306.67 & 8.3196 \\
\hline 73.33 & 2.9108 & & 151.67 & 5.0450 & & 230.00 & 6.8313 & & 308.33 & 8.3492 \\
\hline 75.00 & 2.9671 & & 153.33 & 5.0821 & & 231.67 & 6.8629 & & 310.00 & 8.3821 \\
\hline 76.67 & 3.0208 & & 155.00 & 5.1258 & & 233.33 & 6.8983 & & 311.67 & 8.4096 \\
\hline 78.33 & 3.0713 & & 156.67 & 5.1671 & & 235.00 & 6.9333 & & 313.33 & 8.4238 \\
\hline 80.00 & 3.1229 & & 158.33 & 5.2113 & & 236.67 & 6.9683 & & & \\
\hline 81.67 & 3.1738 & & 160.00 & 5.2504 & & 238.33 & 7.0021 & & & \\
\hline 83.33 & 3.2279 & & 161.67 & 5.2938 & & 240.00 & 7.0367 & & & \\
\hline 85.00 & 3.2775 & & 163.33 & 5.3408 & & 241.67 & 7.0696 & & & \\
\hline 86.67 & 3.3254 & & 165.00 & 5.3871 & & 243.33 & 7.1042 & & & \\
\hline 88.33 & 3.3708 & & 166.67 & 5.4288 & & 245.00 & 7.1375 & & & \\
\hline 90.00 & 3.4204 & & 168.33 & 5.4667 & & 246.67 & 7.1713 & & & \\
\hline 91.67 & 3.4713 & & 170.00 & 5.5067 & & 248.33 & 7.2042 & & & \\
\hline 93.33 & 3.5221 & & 171.67 & 5.5458 & & 250.00 & 7.2379 & & & \\
\hline 95.00 & 3.5683 & & 173.33 & 5.5854 & & 251.67 & 7.2712 & & & \\
\hline 96.67 & 3.6129 & & 175.00 & 5.6250 & & 253.33 & 7.3050 & & & \\
\hline 98.33 & 3.6571 & & 176.67 & 5.6671 & & 255.00 & 7.3350 & & & \\
\hline 100.00 & 3.7021 & & 178.33 & 5.7046 & & .256 .67 & 7.3642 & & & \\
\hline 101.67 & 3.7463 & 次 & 180.00 & 5.7396 & & 258.33 & 7.3917 & & & \\
\hline
\end{tabular}


HNF-SD-SNF-CN-035, Rev. 0

Table C8. Thermo-Gravimetric Analysis Data for $K$ Basin Fuel Sample Run 20 at $250^{\circ} \mathrm{C}$. (Page 1 of 2)

RUN NUMBER 20 AT $250 \mathrm{C}$

\begin{tabular}{|c|c|c|c|c|c|c|c|}
\hline $\begin{array}{c}\text { TIME } \\
\text { (minutes) }\end{array}$ & $\begin{array}{c}\text { Wt Gain } \\
(\mathrm{mg})\end{array}$ & $\begin{array}{c}\text { TIME } \\
\text { (minutes) }\end{array}$ & $\begin{array}{l}\text { Wt Gain } \\
\text { (mg) }\end{array}$ & $\begin{array}{c}\text { TIME } \\
\text { (minutes) }\end{array}$ & $\begin{array}{l}\text { Wt Gain } \\
\text { (mg) }\end{array}$ & $\begin{array}{c}\text { TIME } \\
\text { (minutes) }\end{array}$ & $\begin{array}{c}\text { Wt Gain } \\
\text { (mg) }\end{array}$ \\
\hline 24.98 & 0.68 & 47.94 & 4.18 & 70.90 & 7.053 & 93.86 & 9.692 \\
\hline 25.45 & 0.76 & 48.41 & 4.25 & 71.37 & 7.105 & 94.33 & 9.742 \\
\hline 25.93 & 0.84 & 48.89 & 4.07 & 71.85 & 7.167 & 94.81 & 9.792 \\
\hline 26.41 & 0.92 & 49.37 & 4.14 & 72.33 & 7.225 & 95.29 & 9.842 \\
\hline 26.89 & 0.97 & 49.85 & 4.22 & 72.81 & 7.285 & 95.77 & 9.896 \\
\hline 27.37 & 1.05 & 50.33 & 4.27 & 73.29 & 7.347 & 96.25 & 9.935 \\
\hline 27.85 & 1.12 & 50.81 & 4.35 & 73.77 & 7.404 & 96.73 & 9.984 \\
\hline 28.32 & 1.19 & 51.28 & 4.42 & 74.24 & 7.464 & 97.20 & 10.034 \\
\hline 28.80 & 1.26 & 51.76 & $4 . \overline{49}$ & 74.72 & 7.522 & 97.68 & 10.087 \\
\hline 29.28 & 1.34 & 52.24 & 4.56 & 75.20 & 7.600 & 98.16 & 10.135 \\
\hline 29.76 & 1.41 & 52.72 & 4.62 & 75.68 & 7.650 & 98.64 & 10.185 \\
\hline 30.24 & 1.48 & 53.20 & 4.69 & 76.16 & 7.699 & 99.12 & 10.234 \\
\hline 30.72 & 1.56 & 53.68 & 4.76 & 76.64 & 7.746 & 99.60 & 10.284 \\
\hline 31.19 & 1.64 & 54.15 & 4.83 & 77.11 & 7.804 & 100.07 & 10.331 \\
\hline 31.67 & 1.71 & 54.63 & 4.89 & 77.59 & 7.867 & 100.55 & 10.369 \\
\hline 32.15 & 1.79 & 55.11 & 4.96 & 78.07 & 7.920 & 101.03 & 10.429 \\
\hline 32.63 & 1.87 & 55.59 & 5.03 & 78.55 & 7.976 & 101.51 & 10.478 \\
\hline 33.11 & 1.94 & 56.07 & 5.09 & 79.03 & 8.030 & 101.99 & 10.532 \\
\hline 33.59 & 2.02 & 56.55 & 5.16 & 79.51 & 8.097 & 102.47 & 10.585 \\
\hline 34.06 & 2.09 & 57.02 & 5.23 & 79.98 & 8.154 & 102.94 & 10.622 \\
\hline 34.54 & 2.17 & 57.50 & 5.30 & 80.46 & 8.197 & 103.42 & 10.674 \\
\hline 35.02 & 2.24 & 57.98 & 5.36 & 80.94 & 8.260 & 103.90 & 10.722 \\
\hline 35.50 & 2,31 & 58.46 & 5.43 & 81.42 & 8.314 & 104.38 & 10.771 \\
\hline 35.98 & 2.38 & 58.94 & 5.50 & 81.90 & 8.374 & 104.86 & 10.821 \\
\hline 36.46 & 2.46 & 59.42 & 5.58 & 82.38 & 8.427 & 105.34 & 10.867 \\
\hline 36.93 & 2.52 & 59.89 & 5.63 & 82.85 & 8.481 & 105.81 & 10.923 \\
\hline 37.41 & 2.59 & 60.37 & 5.68 & 83.33 & 8.537 & 106.29 & 10.974 \\
\hline 37.89 & 2.67 & 60.85 & 5.76 & 83.81 & 8.593 & 105.77 & 11.012 \\
\hline 38.37 & 2.74 & 61.33 & 5.82 & 84.29 & 8.643 & 107.25 & 11.061 \\
\hline 38.85 & 2.81 & 61.81 & 5.88 & 84.77 & 8.688 & 107.73 & 11.107 \\
\hline 39.33 & 2.89 & 62.29 & 5.95 & 85.25 & 8.739 & 108.21 & 11.155 \\
\hline 39.80 & 2.96 & 62.76 & 6.01 & 85.72 & 8.806 & 108.68 & 11.201 \\
\hline 40.28 & 3.02 & 63.24 & 6.07 & 86.20 & 8.849 & 109.16 & 11.248 \\
\hline 40.76 & 3.10 & 63.72 & 6.13 & 86.68 & 8.903 & 109.64 & 11.295 \\
\hline 41.24 & 3.17 & 64.20 & 6.19 & 87.16 & 8.965 & 110.12 & 11.343 \\
\hline 41.72 & 3.25 & 64.68 & 6.26 & 87.64 & 9.017 & 110.60 & 11.378 \\
\hline 42.20 & 3.32 & 65.16 & 6.34 & 88.12 & 9.060 & 111.08 & 11.437 \\
\hline 42.67 & 3.40 & 65.63 & 6.41 & 88.59 & 9.111 & 111.55 & 11.489 \\
\hline 43.15 & 3.48 & 66.11 & 6.46 & 89.07 & 9.164 & 112.03 & 11.531 \\
\hline 43.63 & 3.56 & 66.59 & 6.52 & 89.55 & 9.214 & 112.51 & 11.577 \\
\hline 44.11 & 3.62 & 67.07 & 6.58 & 90.03 & 9.281 & 112.99 & 11.622 \\
\hline 44.59 & 3.69 & 67.55 & 6.65 & 90.51 & 9.321 & 113.47 & 11.667 \\
\hline 45.07 & 3.76 & 68.03 & 6.68 & 90.99 & 9.373 & 113.95 & 11.712 \\
\hline 45.54 & 3.82 & 68.50 & 6.75 & 91.46 & 9.438 & 114.42 & 11.757 \\
\hline 46.02 & 3.90 & 68.98 & 6.81 & 91.94 & 9.480 & 114.90 & 11.803 \\
\hline 46.50 & 3.97 & 69.46 & 8.87 & 92.42 & 9.530 & 115.38 & 11.849 \\
\hline 46.98 & 4.04 & 69.94 & 6.94 & 92.90 & 9.581 & 115.86 & 11.895 \\
\hline 47.46 & 4.11 & 70.42 & 6.98 & 93.38 & 9.640 & 116.34 & 11.942 \\
\hline
\end{tabular}


Table C8. Thermo-Gravimetric Analysis Data for K Basin Fue 7 Sample Run 20 at $250^{\circ} \mathrm{C}$. (Page 2 of 2)

\begin{tabular}{|c|c|c|c|c|c|}
\hline \multicolumn{6}{|c|}{ RUN NUMBER 20 AT 250 C (cont.) } \\
\hline $\begin{array}{c}\text { TIME } \\
\text { (minutes) }\end{array}$ & $\begin{array}{c}\text { Wt Gain } \\
(\mathrm{mg})\end{array}$ & $\begin{array}{c}\text { TIME } \\
\text { (minutes) }\end{array}$ & $\begin{array}{c}\text { Wt Gain } \\
(\mathrm{mg})\end{array}$ & $\begin{array}{c}\text { TIME } \\
\text { (minutes) } \\
\end{array}$ & $\begin{array}{l}\text { Wt Gain } \\
(\mathrm{mg})\end{array}$ \\
\hline 116.82 & 11.987 & 139.78 & 14.085 & 162.74 & 15.990 \\
\hline 117.29 & 12.034 & 140.25 & 14.119 & 163.21 & 16.030 \\
\hline 117.77 & 12.078 & 140.73 & 14.154 & 163.69 & 16.068 \\
\hline 118.25 & 12.124 & 141.21 & 14.195 & 164.17 & 16.104 \\
\hline 118.73 & 12.170 & 141.69 & 14.236 & 164.65 & 16.145 \\
\hline 119.21 & 12.213 & 142.17 & 14.278 & 165.13 & 16.185 \\
\hline 119.69 & 12.259 & 142.65 & 14.319 & 165.61 & 16.234 \\
\hline 120.16 & 12.313 & 143.12 & 14.361 & $166 . \overline{08}$ & 16.270 \\
\hline 120.64 & 12.340 & 143.60 & 14.402 & & \\
\hline 121.12 & 12.390 & 144.08 & 14.440 & & \\
\hline 121.60 & 12.437 & 144.56 & 14.483 & & \\
\hline 122.08 & 12.484 & 145.04 & 14.525 & & \\
\hline 122.56 & 12.528 & 145.52 & 14.567 & & \\
\hline 123.03 & 12.570 & 145.99 & 14.608 & & \\
\hline 123.51 & 12.614 & 146.47 & 14.648 & & \\
\hline 123.99 & 12.659 & 146.95 & 14.685 & & \\
\hline 124.47 & 12.702 & $\overline{147.43}$ & 14.725 & & \\
\hline 124.95 & 12.747 & 147.91 & 14.765 & & \\
\hline 125.43 & 12.790 & 148.39 & 14.810 & & \\
\hline 125.90 & 12.832 & 148.86 & 14.860 & & \\
\hline 126.38 & 12.875 & 149.34 & 14.900 & & \\
\hline 126.86 & 12.920 & 149.82 & 14.938 & & \\
\hline 127.34 & 12.963 & 150.30 & 14.968 & & \\
\hline 127.82 & 13.007 & 150.78 & 15.008 & & \\
\hline 128.30 & 13.051 & 151.26 & 15.059 & & \\
\hline 128.77 & 13.095 & 151.73 & 15.100 & & \\
\hline 129.25 & 13.138 & 152.21 & 15.142 & & \\
\hline 129.73 & 13.193 & 152.69 & 15.179 & & \\
\hline 130.21 & 13.229 & 153.17 & 15.218 & & \\
\hline 130.69 & 13.265 & 153.65 & 15.259 & & . \\
\hline 131.17 & 13.320 & 154.13 & 15.300 & & \\
\hline 131.64 & 13.354 & 154.60 & 15.339 & & \\
\hline 132.12 & 13.397 & 155.08 & 15.379 & & \\
\hline 132.60 & 13.439 & 155.56 & $15.41 \overline{6}$ & & \\
\hline 133.08 & 13.485 & 156.04 & 15.456 & & \\
\hline 133.56 & 13.538 & 156.52 & 15.495 & & \\
\hline 134.04 & 13.581 & 157.00 & 15.535 & & \\
\hline 134.51 & 13.625 & 157.47 & 15.573 & & \\
\hline 134.99 & 13.661 & 157.95 & 15.611 & & \\
\hline 135.47 & 13.705 & 158.43 & 15.651 & & \\
\hline 135.95 & 13.749 & 158.91 & 15.691 & & \\
\hline 136.43 & 13.792 & 159.39 & 15.730 & & \\
\hline 136.91 & 13.837 & 159.87 & 15.770 & & \\
\hline 137.38 & 13.865 & 160.34 & 15.795 & & \\
\hline 137.86 & 13.916 & 160.82 & 15.838 & & \\
\hline 138.34 & 13.961 & 161.30 & 15.876 & & \\
\hline 138.82 & 14.003 & 161.78 & 15.910 & & \\
\hline 139.30 & 14.043 & 162.26 & 15.950 & & \\
\hline
\end{tabular}


Table C9. Thermo-Gravimetric Analysis Data for $K$ Basin Fue 7 Sample Run 21 a at $300{ }^{\circ} \mathrm{C}$. (Page 1 of 3 )

\begin{tabular}{|c|c|c|c|c|c|c|c|}
\hline \multicolumn{8}{|c|}{ RUN NUMBER 21a AT $300 \mathrm{C}$} \\
\hline $\begin{array}{l}\text { TIME } \\
\text { (minutes) }\end{array}$ & $\begin{array}{c}\text { wt change } \\
(\mathrm{mg})\end{array}$ & $\begin{array}{l}\text { TIME } \\
\text { (minutes) }\end{array}$ & $\begin{array}{c}\text { wt change } \\
\text { (mg) }\end{array}$ & $\begin{array}{l}\text { IME } \\
\text { (minutes) }\end{array}$ & $\begin{array}{c}\text { wt change } \\
(\mathrm{mg})\end{array}$ & $\begin{array}{l}\text { TIME } \\
\text { (minutes) }\end{array}$ & $\begin{array}{c}\text { wt change } \\
\text { (mg) }\end{array}$ \\
\hline 24.99 & 0.343 & $\overline{449.49}$ & 1.557 & 73.99 & 2.822 & 98.49 & 3.860 \\
\hline 25.48 & 0.301 & 49.98 & 1.543 & 74.48 & 2.832 & 98.98 & 3.835 \\
\hline 25.97 & 0.388 & 50.47 & 1.540 & 74.97 & 2.834 & 99.47 & 3.809 \\
\hline 26.46 & 0.348 & 50.96 & 1.546 & 75.46 & 2.873 & 99.96 & 3.913 \\
\hline 26.95 & 0.400 & 51.45 & 1.621 & 75.95 & 2.855 & 100.45 & 3.916 \\
\hline 27.44 & 0.382 & 51.94 & 1.599 & 76.44 & 2.899 & 100.94 & 3.858 \\
\hline 27.93 & 0.374 & 52.43 & 1.576 & 76.93 & 2.859 & 101.43 & 3.848 \\
\hline 28.42 & 0.605 & 52.92 & 1.593 & 77.42 & 2.913 & 101.92 & 3.789 \\
\hline 28.91 & 0.455 & 53.41 & 1.655 & 77.91 & 2.871 & 102.41 & 3.828 \\
\hline 29.40 & 0.789 & 53.90 & 1.666 & 78.40 & 2.898 & 102.90 & 3.773 \\
\hline 29.89 & 0.416 & 54.39 & 1.668 & 78.89 & 2.928 & 103.39 & 3.865 \\
\hline 30.38 & 0.548 & 54.88 & 1.703 & 79.38 & 2.922 & 103.88 & 3.823 \\
\hline 30.87 & 0.531 & 55.37 & 1.699 & 79.87 & 2.940 & 104.37 & 3.878 \\
\hline 31.36 & 0.825 & 55.86 & 1.832 & 80.36 & 2.943 & 104.86 & 3.823 \\
\hline 31.85 & 0.663 & 56.35 & 2.034 & 80.85 & 2.934 & 105.35 & 3.643 \\
\hline 32.34 & 0.873 & 56.84 & 2.080 & 81.34 & 2.999 & 105.84 & 3.764 \\
\hline 32.83 & 0.775 & 57.33 & 2.114 & 81.83 & 3.075 & 106.33 & 3.818 \\
\hline 33.32 & 0.747 & 57.82 & 2.020 & 82.32 & 3.073 & 106.82 & 3.869 \\
\hline 33.81 & 0.980 & 58.31 & 2.081 & 82.81 & 3.184 & 107.31 & 3.883 \\
\hline 34.30 & 0.855 & 58.80 & 2.064 & 83.30 & 3.182 & 107.80 & 3.949 \\
\hline 34.79 & 0.863 & 59.29 & 2.320 & 83.79 & 3.173 & 108.29 & 3.980 \\
\hline 35.28 & 0.799 & 59.78 & 2.270 & 84.28 & 3.501 & 108.78 & 3.994 \\
\hline 35.77 & 0.815 & 60.27 & 2.145 & 84.77 & 3.611 & 109.27 & 4.056 \\
\hline 36.26 & 0.732 & 60.76 & 2.080 & 85.26 & 2.944 & 109.76 & 4.070 \\
\hline 36.75 & 0.784 & 61.25 & 1.949 & 85.75 & 3.191 & 110.25 & 4.030 \\
\hline 37.24 & 0.926 & 61.74 & 1.928 & 86.24 & 3.221 & 110.74 & 4.092 \\
\hline $37: 73$ & 0.519 & 62.23 & 2.093 & 86.73 & 3.518 & 111.23 & 3.925 \\
\hline 38.22 & 0.938 & 62.72 & 2.074 & 87.22 & 3.494 & 111.72 & 3.950 \\
\hline 38.71 & 1.519 & 63.21 & 2.053 & 87.71 & 3.531 & 112.21 & 3.995 \\
\hline 39.20 & 1.014 & 63.70 & 2.107 & 88.20 & 3.568 & 112.70 & 3.960 \\
\hline 39.69 & 0.629 & 64.19 & 2.236 & 88.69 & 3.609 & 113.19 & 3.997 \\
\hline 40.18 & 1.400 & 64.68 & 2.174 & 89.18 & 3.571 & 113.68 & 4.072 \\
\hline 40.67 & 1.213 & 65.17 & 2.167 & 89.67 & 3.601 & 114.17 & 4.032 \\
\hline 41.16 & 0.975 & 65.66 & 2.290 & 90.16 & 3.489 & 114.66 & 4.095 \\
\hline 41.65 & 1.428 & 66.15 & 2.774 & 90.65 & 3.560 & 115.15 & 4.167 \\
\hline 42.14 & 1.524 & 66.64 & 2.817 & 91.14 & 3.582 & 115.64 & 4.135 \\
\hline 42.63 & 1.550 & 67.13 & 2.820 & 91.63 & 3.620 & 116.13 & 4.228 \\
\hline 43.12 & 1.562 & 67.62 & 2.828 & 92.12 & 3.583 & 116.62 & 4.252 \\
\hline 43.61 & 1.585 & 68.11 & 2.808 & 92.61 & 3.558 & 117.11 & 4.300 \\
\hline 44.10 & 1.570 & 68.60 & 2.809 & 93.10 & 3.607 & 117.60 & 4.234 \\
\hline 44.59 & 1.580 & 69.09 & 2.795 & 93.59 & 3.640 & 118.09 & 4.230 \\
\hline 45.08 & 1.569 & 69.58 & 2.819 & 94.08 & 3.558 & 118.58 & 4.328 \\
\hline 45.57 & 1.578 & 70.07 & 2.7 .63 & 94.57 & 3.509 & 119.07 & 4.194 \\
\hline 46.06 & 1.588 & 70.56 & 2.808 & 95.06 & 3.528 & 119.56 & 4.215 \\
\hline 46.55 & 1.368 & 71.05 & 2.857 & 95.55 & 3.661 & 120.05 & 4.247 \\
\hline 47.04 & 1.566 & 71.54 & 2.821 & 96.04 & 3.566 & 120.54 & 4.253 \\
\hline 47.53 & 1.570 & 72.03 & 2.805 & 96.53 & 3.579 & 121.03 & 4.190 \\
\hline 48.02 & 1.561 & 72.52 & 2.799 & 97.02 & 3.770 & 121.52 & 4.227 \\
\hline 48.51 & 1.545 & 73.01 & 2.859 & 97.51 & 3.796 & 122.01 & 4.254 \\
\hline 49.00 & 1.563 & 73.50 & 2.826 & 98.00 & 3.795 & 122.50 & 4.217 \\
\hline
\end{tabular}


Table C9. Thermo-Gravimetric Analys is Data for $K$ Basin Fuel Sample Run 2la at $300^{\circ} \mathrm{C}$. (Page 2 of 3 )

\begin{tabular}{|c|c|c|c|c|c|c|c|}
\hline \multicolumn{8}{|c|}{ RUN NUMBER 21a AT $300 \mathrm{C}$ (cont.) } \\
\hline $\begin{array}{l}\text { TIME } \\
\text { (minutes) }\end{array}$ & $\begin{array}{c}\text { wt change } \\
\text { (mg) }\end{array}$ & $\begin{array}{l}\text { TIME } \\
\text { (minutes) }\end{array}$ & $\begin{array}{c}\text { wt change } \\
\text { (mg) }\end{array}$ & $\begin{array}{l}\text { TIME } \\
\text { (minutes) }\end{array}$ & $\begin{array}{c}\text { wt change } \\
\text { (mg) }\end{array}$ & $\begin{array}{l}\text { TIME } \\
\text { (minutes) }\end{array}$ & $\begin{array}{c}\text { wt change } \\
(\mathrm{mg})\end{array}$ \\
\hline 122.99 & 4.221 & 147.49 & 4.473 & 171.99 & 5.270 & 196.49 & 5.939 \\
\hline 123.48 & 4.143 & 147.98 & 4.440 & 172.48 & 5.013 & 196.98 & 5.934 \\
\hline 123.97 & 4.148 & 148.47 & 4.438 & 172.97 & 5.274 & 197.47 & 5.974 \\
\hline 124.46 & $4: 176$ & 148.96 & 4.446 & 173.46 & 5.305 & 197.96 & 5.943 \\
\hline 124.95 & 4.242 & 149.45 & 4.457 & 173.95 & 5.300 & 198.45 & 5.952 \\
\hline 125.44 & 4.026 & 149.94 & 4.408 & 174.44 & 5.421 & 198.94 & 5.957 \\
\hline 125.93 & 4.248 & 150.43 & 4.423 & 174.93 & 5.410 & 199.43 & 5.961 \\
\hline 126.42 & 4.179 & 150.92 & 4.449 & 175.42 & 5.433 & 199.92 & 5.980 \\
\hline 126.91 & 4.056 & 151.41 & 4.447 & 175.91 & 5.633 & 200.41 & 5.972 \\
\hline 127.40 & 4.277 & 151.90 & 4.480 & 176.40 & 5.791 & 200.90 & 5.969 \\
\hline 127.89 & 4.170 & 152.39 & 4.475 & 176.89 & 5.679 & 201.39 & 6.605 \\
\hline .128 .38 & 4.026 & 152.88 & 4.444 & 177.38 & 5.755 & 201.88 & 5.893 \\
\hline 128.87 & 4.080 & 153.37 & 4.455 & 177.87 & 5.791 & 202.37 & 5.980 \\
\hline 129.36 & 4.070 & 153.86 & 4.465 & 178.36 & 5.766 & 202.86 & 5.948 \\
\hline 129.85 & 4.039 & 154.35 & 4.456 & 178.85 & 4.895 & 203.35 & 5.918 \\
\hline 130.34 & 4.079 & 154.84 & 4.477 & 179.34 & 5.240 & 203.84 & 7.378 \\
\hline 130.83 & 4.127 & 155.33 & 4.469 & 179.83 & 5.501 & 204.33 & 7.192 \\
\hline 131.32 & 4.114 & 155.82 & 4.485 & 180.32 & 5.513 & 204.82 & 6.574 \\
\hline 131.81 & 4.190 & 156.31 & 4.491 & 180.81 & 5.612 & 205.31 & 6.025 \\
\hline 132.30 & 4.217 & 156.80 & 4.493 & 181.30 & 5.675 & 205.80 & 6.886 \\
\hline 132.79 & 4.250 & 157.29 & 4.483 & 181.79 & 5.683 & 206.29 & 7.914 \\
\hline 133.28 & 4.234 & 157.78 & 4.477 & 182.28 & 5.713 & 206.78 & 7.846 \\
\hline 133.77 & 4.281 & 158.27 & 4.494 & 182.77 & 5.712 & 207.27 & 8.135 \\
\hline 134.26 & 4.485 & 158.76 & 4.570 & 183.26 & 5.735 & 207.76 & 8.194 \\
\hline 134.75 & 4.390 & 159.25 & 4.536 & 183.75 & 5.799 & 208.25 & 8.180 \\
\hline 135.24 & 4.271 & 159.74 & 4.522 & 184.24 & 5.810 & 208.74 & 8.183 \\
\hline 135.73 & 4.435 & 160.23 & 4.580 & 184.73 & 5.813 & 209.23 & 8.274 \\
\hline 136.22 & 4.353 & 160.72 & 4.648 & 185.22 & 5.811 & 209.72 & 8.243 \\
\hline 136.71 & 4.370 & 161.21 & 4.548 & 185.71 & 5.834 & 210.21 & 8.185 \\
\hline 137.20 & 4.234 & 161.70 & 4.581 & 186.20 & 5.837 & 210.70 & 8.229 \\
\hline 137.69 & 4.105 & 162.19 & 4.540 & 186.69 & 5.830 & 211.19 & 8.210 \\
\hline 138.18 & 4.223 & 162.68 & 4.533 & 187.18 & 5.841 & 211.68 & 8.257 \\
\hline 138.67 & 4.406 & 163.17 & 4.540 & 187.67 & 5.904 & 212.17 & 8.131 \\
\hline 139.16 & 4.225 & 163.66 & 4.485 & 188.16 & 5.903 & 212.66 & 8.222 \\
\hline 139.65 & 4.369 & 164.15 & 4.518 & 188.65 & 5.945 & 213.15 & 8.252 \\
\hline 140.14 & 4.339 & 164.64 & 4.561 & 189.14 & 5.918 & 213.64 & 8.278 \\
\hline 140.63 & 4.313 & 165.13 & 4.569 & 189.63 & 5.943 & 214.13 & 8.329 \\
\hline 141.12 & 4.343 & 165.62 & 4.573 & 190.12 & 5.995 & 214.62 & 8.251 \\
\hline 141.61 & 4.358 & 166.11 & 4.600 & 190.61 & 5.958 & 215.11 & 8.260 \\
\hline 142.10 & 4.414 & 166.60 & 4.555 & 191.10 & 5.955 & 215.60 & 8.325 \\
\hline 142.59 & 4.409 & 167.09 & 4.574 & 191.59 & 5.938 & 216.09 & 8.356 \\
\hline 143.08 & 4.425 & 167.58 & 4.576 & 192.08 & 5.907 & 216.58 & 8.330 \\
\hline 143.57 & 4.336 & 168.07 & 4.735 & 192.57 & 5.921 & 217.07 & 8.281 \\
\hline 144.06 & 4.388 & 168.56 & 4.627 & 193.06 & 5.968 & 217.56 & 8.305 \\
\hline 144.55 & 4.426 & 169.05 & 4.729 & 193.55 & 5.899 & 218.05 & 8.315 \\
\hline 145.04 & 4.399 & 169.54 & 4.680 & 194.04 & 5.930 & 218.54 & 8.318 \\
\hline 145.53 & 4.435 & 170.03 & 4.788 & 194.53 & 5.935 & 219.03 & 8.387 \\
\hline 146.02 & 4.462 & 170.52 & 4.710 & 195.02 & 5.899 & 219.52 & 8.400 \\
\hline 146.51 & 4.457 & 171.01 & 5.262 & 195.51 & 5.943 & 220.01 & 8.382 \\
\hline 147.00 & 4.451 & 171.50 & 4.915 & 196.00 & 5.924 & 220.50 & 8.353 \\
\hline
\end{tabular}


Table C9. Thermo-Gravimetric Analys is Data for $K$ Basin Fuel Sample Run 21a at $300{ }^{\circ} \mathrm{C}$. (Page 3 of 3 )

\begin{tabular}{|c|c|c|c|c|c|c|c|}
\hline \multicolumn{8}{|c|}{ RUN NUMBER 21a AT $300 \mathrm{C}$ (cont.) } \\
\hline TIME & wt change & TIME & wt change & TIME & wt change & TIME & wt change \\
\hline (minutes) & (mg) & (minutes) & $(\mathrm{mg})$ & (minutes) & (mg) & (minutes) & (mg) \\
\hline 220.99 & 8.453 & 245.49 & 8.550 & 269.99 & 8.570 & 294.49 & 8.563 \\
\hline 221.48 & 8.412 & 245.98 & 8.567 & 270.48 & 8.574 & 294.98 & 8.553 \\
\hline 221.97 & 8.370 & 246.47 & 8.524 & 270.97 & 8.552 & 295.47 & 8.540 \\
\hline 222.46 & 8.339 & 246.96 & 8.570 & 271.46 & 8.572 & 295.96 & 8.550 \\
\hline 222.95 & 8.416 & 247,45 & 8.563 & 271.95 & 8.583 & 296.45 & 8.540 \\
\hline 223.44 & 8.399 & 247.94 & 8.550 & 272.44 & 8.562 & 296.94 & 8.539 \\
\hline 223.93 & 8.425 & 248.43 & 8.522 & 272.93 & 8.569 & 297.43 & 8.573 \\
\hline 224.42 & 8.450 & 248.92 & 8.582 & 273.42 & 8.573 & 297.92 & 8.576 \\
\hline 224.91 & 8.447 & 249.41 & 8.563 & 273.91 & 8.561 & 298.41 & 8.579 \\
\hline 225.40 & 8.434 & 249.90 & 8.568 & 274.40 & 8.554 & 298.90 & 8.555 \\
\hline 225.89 & 8.458 & 250.39 & 8.550 & 274.89 & 8.559 & 299.39 & 8.568 \\
\hline 226.38 & 8.485 & 250.88 & 8.540 & 275.38 & 8.573 & 299.88 & 8.562 \\
\hline 226.87 & 8.448 & 251.37 & 8.578 & 275.87 & 8.576 & 300.37 & 8.556 \\
\hline 227.36 & 8.477 & 251.86 & 8.582 & 276.36 & 8.582 & 300.86 & 8.544 \\
\hline 227.85 & 8.521 & 252.35 & 8.570 & 276.85 & 8.566 & 301.35 & 8.573 \\
\hline 228.34 & 8.507 & 252.84 & 8.558 & 277.34 & 8.546 & 301.84 & 8.565 \\
\hline 228.83 & 8.545 & 253.33 & 8.564 & 277.83 & 8.567 & 302.33 & 8.551 \\
\hline 229.32 & 8.493 & 253.82 & 8.537 & 278.32 & 8.575 & 302.82 & 8.583 \\
\hline 229.81 & 8.543 & 254.31 & 8.533 & 278.81 & 8.555 & 303.31 & 8.581 \\
\hline 230.30 & 8.589 & 254.80 & 8.559 & 279.30 & 8.558 & 303.80 & 8.540 \\
\hline 230.79 & 8.565 & 255.29 & 8.573 & 279.79 & 8.585 & 304.29 & 8.577 \\
\hline 231.28 & 8.532 & 255.78 & 8.587 & 280.28 & 8.577 & 304.78 & 8.583 \\
\hline 231.77 & 8.548 & 256.27 & 8.576 & 280.77 & 8.552 & 305.27 & 8.563 \\
\hline 232.26 & 8.515 & 256.76 & 8.574 & 281.26 & 8.560 & 305.76 & 8.566 \\
\hline 232.75 & 8.543 & 257.25 & 8.573 & 281.75 & 8.553 & 306.25 & 8.566 \\
\hline 233.24 & 8.549 & 257.74 & 8.553 & 282.24 & 8.558 & 306.74 & 8.571 \\
\hline 233.73 & 8.538 & 258.23 & 8.567 & 282.73 & 8.565 & 307.23 & 8.573 \\
\hline 234.22 & 8.573 & 258.72 & 8.577 & 283.22 & 8.572 & 307.72 & 8.577 \\
\hline 234.71 & 8.584 & 259.21 & 8.523 & 283.71 & 8.570 & 308.21 & 8.566 \\
\hline 235.20 & 8.549 & 259.70 & 8.557 & 284.20 & 8.540 & 308.70 & 8.581 \\
\hline 235.69 & 8.430 & 260.19 & 8.574 & 284.69 & 8.571 & 309.19 & 8.565 \\
\hline 236.18 & 8.519 & 260.68 & 8.552 & 285.18 & 8.460 & 309.68 & 8.561 \\
\hline 236.67 & 8.545 & 261.17 & 8.563 & 285.67 & 8.538 & 310.17 & 8.562 \\
\hline 237.16 & 8.515 & 261.66 & 8.581 & 286.16 & 8.549 & 310.66 & 8.545 \\
\hline 237.65 & 8.525 & 262.15 & 8.569 & 286.65 & 8.554 & 311.15 & 8.590 \\
\hline 238.14 & 8.557 & 262.64 & 8.545 & 287.14 & 8.537 & 311.64 & 8.566 \\
\hline 238.63 & 8.515 & 263.13 & 8.547 & 287.63 & 8.549 & 312.13 & 8.562 \\
\hline 239.12 & 8.480 & 263.62 & 8.581 & 288.12 & 8.566 & 312.62 & 8.564 \\
\hline 239.61 & 8.538 & 264.11 & 8.569 & 288.61 & 8.557 & 313.11 & 8.588 \\
\hline 240.10 & 8.530 & 264.60 & 8.549 & 289.10 & 8.552 & 313.60 & 8.560 \\
\hline 240.59 & 8.522 & 265.09 & 8.562 & 289.59 & 8.557 & 314.09 & 8.587 \\
\hline 241.08 & 8.535 & 265.58 & 8.564 & 290.08 & 8.564 & 314.58 & .8 .579 \\
\hline 241.57 & 8.523 & 266.07 & 8.542 & 290.57 & 8.560 & & \\
\hline 242.06 & 8.547 & 266.56 & 8.568 & 291.06 & 8.531 & & \\
\hline 242.55 & 8.525 & 267.05 & 8.564 & 291.55 & 8.559 & & \\
\hline 243.04 & 8.505 & 267.54 & 8.552 & 292.04 & 8.570 & & \\
\hline 243.53 & 8.530 & 268.03 & 8.561 & 292.53 & 8.525 & & \\
\hline 244.02 & 8.531 & 268.52 & 8.562 & 293.02 & 8.560 & & \\
\hline 244.51 & 8.533 & 269.01 & 8.557 & 293.51 & 8.569 & & \\
\hline 245.00 & 8.552 & 269.50 & 8.553 & 294.00 & 8.559 & & \\
\hline
\end{tabular}


Table C10. Thermo-Gravimetric Analys is Data

for $K$ Basin Fue] Sample Run 22 at $350{ }^{\circ} \mathrm{C}$.

\begin{tabular}{|c|c|c|c|c|c|c|c|}
\hline \multicolumn{8}{|c|}{ RUN NUMBER 22 AT $350 \mathrm{C}$} \\
\hline $\begin{array}{c}\text { Time } \\
\text { (minutes) }\end{array}$ & $\begin{array}{c}\text { Wt Gain } \\
\text { (gm) }\end{array}$ & $\begin{array}{c}\text { Time } \\
\text { (minutes) }\end{array}$ & $\begin{array}{l}\text { Wt Gain } \\
\text { (gm) }\end{array}$ & $\begin{array}{cc}\text { Time } \\
\text { (minutes) } \\
\end{array}$ & $\begin{array}{l}\text { Wt Gain } \\
(\mathrm{gm})\end{array}$ & $\begin{array}{c}\text { Time } \\
\text { (minutes) }\end{array}$ & $\begin{array}{c}\text { Wt Gain } \\
(\mathrm{gm})\end{array}$ \\
\hline 20.00 & 0.4354 & 42.36 & 3.1188 & 64.71 & 2.8479 & 87.07 & 5.6842 \\
\hline 20.46 & 0.3863 & 42.82 & 1.8908 & 65.18 & 2.8679 & 87.54 & 5.8933 \\
\hline 20.93 & 0.7558 & 43.29 & 2.1488 & 65.65 & 2.8933 & 88.01 & 5.7371 \\
\hline 21.39 & 1.1421 & 43.75 & 3.5817 & 66.11 & 3.2363 & 88.47 & 5.9838 \\
\hline 21.86 & 1.1250 & 44.22 & 2.2504 & 66.58 & 3.0179 & 88.94 & 5.9058 \\
\hline 22.32 & 1.5221 & 44.68 & 3.0767 & 67.04 & 3.1708 & 89.40 & 5.8333 \\
\hline 22.79 & 1.6933 & 45.15 & 2.2163 & 67.51 & 3.0683 & 89.87 & 5.8654 \\
\hline 23.26 & 1.0729 & 45.62 & 2.5921 & 67.98 & 3.0654 & 90.34 & 5.8804 \\
\hline 23.72 & 1.1125 & 46.08 & 3.3967 & 68.44 & 3.2163 & 90.80 & 5.9746 \\
\hline 24.19 & 1.4613 & 46.55 & 2.4133 & 68.91 & 3.0163 & 91.27 & 7.0233 \\
\hline 24.65 & 1.2458 & 47.01 & 2.4896 & 69.37 & 3.5904 & 91.73 & 6.2796 \\
\hline 25.12 & 1.3821 & 47.48 & 2.1400 & 69.84 & 3.3475 & 92.20 & 5.9454 \\
\hline 25.59 & 1.5550 & 47.94 & 2.2100 & 70.30 & 3.0788 & 92.67 & 6.0667 \\
\hline 26.05 & 1.5863 & 48.41 & 2.3496 & 70.77 & 3.3950 & 93.13 & 6.1092 \\
\hline 26.52 & 1.7379 & 48.88 & 2.3879 & 71.24 & 3.1267 & 93.60 & 6.0496 \\
\hline 26.98 & 1.5971 & 49.34 & 2.3458 & 71.70 & 3.5621 & 94.06 & 6.1896 \\
\hline 27.45 & 1.7013 & 49.81 & 2.4258 & 72.17 & 3.4933 & 94.53 & 5.9633 \\
\hline 27.91 & 1.5704 & 50.27 & 2.3688 & 72.63 & 3.4983 & 94.99 & 6.1567 \\
\hline 28.38 & 1.7788 & 50.74 & 2.4908 & 73.10 & 3.5813 & 95.46 & 7.6200 \\
\hline 28.85 & 1.7692 & 51.21 & 2.3042 & 73.57 & 3.3013 & 95.93 & 12.4910 \\
\hline 29.31 & 1.8204 & 51.67 & 2.3246 & 74.03 & 3.5863 & 96.39 & 12.2660 \\
\hline 29.78 & 1.2646 & 52.14 & 2.4329 & 74.50 & 3.3213 & 96.86 & 12.4500 \\
\hline 30.24 & 1.3858 & 52.60 & 2.5625 & 74.96 & 3.6429 & 97.32 & 12.3510 \\
\hline 30.71 & 1.8213 & 53.07 & 2.4892 & 75.43 & 3.8792 & 97.79 & 12.6070 \\
\hline 31.18 & 1.9721 & 53.54 & 2.5317 & 75.90 & 3.3388 & 98.26 & 18.9500 \\
\hline 31.64 & 1.9933 & 54.00 & 2.5221 & 76.36 & 3.7225 & 98.72 & 18.7740 \\
\hline 32.11 & 1.9392 & 54.47 & 2.5500 & 76.83 & 3.7092 & 99.19 & 18.8180 \\
\hline 32.57 & 1.5121 & 54.93 & 2.5208 & 77.29 & 3.5283 & 99.65 & 17.8760 \\
\hline 33.04 & 1.6358 & 55.40 & 2.6075 & 77.76 & 3.6125 & 100.12 & 16.5340 \\
\hline 33.50 & 1.7217 & 55.86 & 2.5513 & 78.22 & 3.6521 & 100.58 & 14.3890 \\
\hline 33.97 & 2.0438 & 56.33 & 2.5600 & 78.69 & 3.8033 & 101.05 & 13.7810 \\
\hline 34.44 & 2.1696 & 56.80 & 2.6221 & 79.16 & 3.8383 & 101.52 & 13.0080 \\
\hline 34.90 & 2.2200 & 57.26 & 2.6400 & 79.62 & 3.8283 & 101.98 & 12.8060 \\
\hline 35.37 & 2.6171 & 57.73 & 2.5867 & 80.09 & 3.6683 & 102.45 & 13.4630 \\
\hline 35.83 & 2.8646 & 58.19 & 2.6846 & 80.55 & 3.6829 & 102.91 & 13.1180 \\
\hline 36.30 & 2.8000 & 58.66 & 2.7083 & 81.02 & 3.6675 & 103.38 & 13.0260 \\
\hline 36.76 & 2.6438 & 59.13 & 2.6904 & 81.49 & 4.1263 & 103.85 & 13.1380 \\
\hline 37.23 & 2.7871 & 59.59 & 2.6546 & 81.95 & 5.8417 & 104.31 & 12.9020 \\
\hline 37.70 & 2.6121 & 60.06 & 2.6950 & 82.42 & 5.6375 & 104.78 & 14.0660 \\
\hline 38.16 & 2.7775 & 60.52 & 2.7338 & 82.88 & 5.5100 & 105.24 & 13.3570 \\
\hline 38.63 & 2.8504 & 60.99 & 2.8121 & 83.35 & 5.3488 & 105.71 & 18.8400 \\
\hline 39.09 & 2.8817 & 61.45 & 2.8217 & 83.81 & 5.5179 & & \\
\hline 39.56 & 2.6483 & 61.92 & 2.7967 & 84.28 & 5.6992 & & \\
\hline 40.03 & 2.9054 & 62.39 & 2.8567 & 84.75 & 5.6096 & & \\
\hline 40.49 & 2.8500 & 62.85 & 2.8275 & 85.21 & 5.7163 & & \\
\hline 40.96 & 3.0250 & 63.32 & 2.8358 & 85.68 & 5.8050 & & \\
\hline 41.42 & 3.0713 & 63.78 & 2.9775 & 86.14 & 5.6913 & & \\
\hline 41.89 & 2.9375 & 64.25 & 2.7617 & 86.61 & 5.7821 & & \\
\hline
\end{tabular}


Table C11. Thermo-Gravimetric Analysis Data for $K$ Basin Fuel Sample Run 23 at $150{ }^{\circ} \mathrm{C}$. (Page 1 of 3 )

RUN NUMBER 23 AT $150 \mathrm{C}$

\begin{tabular}{|c|c|c|c|c|c|c|c|}
\hline $\begin{array}{c}\text { TIME } \\
\text { (minutes) }\end{array}$ & $\begin{array}{c}\text { wt change } \\
(\mathrm{mg})\end{array}$ & $\begin{array}{c}\text { TIME } \\
\text { (minutes) }\end{array}$ & \begin{tabular}{|c} 
wt change \\
(mg)
\end{tabular} & $\begin{array}{c}\text { TIME } \\
\text { (minutes) }\end{array}$ & $\begin{array}{c}\text { wt change } \\
(\mathrm{mg})\end{array}$ & $\begin{array}{c}\text { TIME } \\
\text { (minutes) }\end{array}$ & \begin{tabular}{|c} 
wt change \\
(mg)
\end{tabular} \\
\hline 25.00 & 0.0687 & 49.00 & 0.1317 & 73.00 & 0.1754 & 97.00 & 0.2100 \\
\hline 25.50 & 0.0662 & 49.50 & 0.1304 & 73.50 & 0.1813 & 97.50 & 0.2158 \\
\hline 26.00 & 0.0687 & 50.00 & 0.1342 & 74.00 & 0.1779 & 98.00 & 0.2192 \\
\hline 26.50 & 0.0717 & 50.50 & 0.1342 & 74.50 & 0.1804 & 98.50 & 0.2229 \\
\hline 27.00 & 0.0738 & 51.00 & 0.1363 & 75.00 & 0.1800 & 99.00 & 0.2183 \\
\hline 27.50 & 0.0742 & 51.50 & 0.1258 & 75.50 & 0.1792 & 99.50 & 0.2229 \\
\hline 28.00 & 0.0808 & 52.00 & 0.1371 & 76.00 & 0.1763 & 100.00 & 0.2221 \\
\hline 28.50 & 0.0758 & 52.50 & 0.1346 & 76.50 & 0.1767 & 100.50 & 0.2225 \\
\hline 29.00 & 0.0771 & 53.00 & 0.1263 & 77.00 & 0.1779 & 101.00 & 0.2267 \\
\hline 29.50 & 0.0787 & 53.50 & 0.1333 & 77.50 & 0.1800 & 101.50 & 0.2242 \\
\hline 30.00 & 0.0771 & 54.00 & 0.1346 & 78.00 & 0.1788 & 102.00 & 0.2238 \\
\hline 30.50 & 0.0858 & 54.50 & 0.1433 & 78.50 & 0.1850 & 102.50 & 0.2233 \\
\hline 31.00 & 0.0900 & 55.00 & 0.1413 & 79.00 & 0.1846 & 103.00 & 0.2254 \\
\hline 31.50 & 0.0650 & 55.50 & 0.1288 & 79.50 & 0.1904 & 103.50 & 0.2275 \\
\hline 32.00 & 0.0738 & 56.00 & 0.1300 & 80.00 & 0.1908 & 104.00 & 0.2250 \\
\hline 32.50 & 0.0796 & 56.50 & 0.1271 & 80.50 & 0.1892 & 104.50 & 0.2246 \\
\hline 33.00 & 0.0812 & 57.00 & 0.1204 & 81.00 & 0.1875 & 105.00 & 0.1842 \\
\hline 33.50 & 0.0892 & 57.50 & 0.1196 & 81.50 & 0.1908 & 105.50 & 0.1971 \\
\hline 34.00 & 0.0888 & 58.00 & 0.1400 & 82.00 & 0.1900 & 106.00 & 0.1871 \\
\hline 34.50 & 0.0908 & 58.50 & 0.1400 & 82.50 & 0.1883 & 106.50 & 0.2025 \\
\hline 35.00 & 0.0867 & 59.00 & 0.1438 & 83.00 & 0.1879 & 107.00 & 0.2083 \\
\hline 35.50 & 0.0987 & 59.50 & 0.1496 & 83.50 & 0.1913 & 107.50 & 0.2029 \\
\hline 36.00 & 0.0962 & 60.00 & 0.1463 & 84.00 & 0.1867 & 108.00 & 0.2063 \\
\hline 36.50 & 0.0863 & 60.50 & 0.1479 & 84.50 & 0.1929 & 108.50 & 0.2050 \\
\hline 37.00 & 0.0954 & 61.00 & 0.1479 & 85.00 & 0.1946 & 109.00 & 0.2054 \\
\hline 37.50 & 0.0975 & 61.50 & 0.1471 & 85.50 & 0.1933 & 109.50 & 0.2133 \\
\hline 38.00 & 0.0979 & 62.00 & 0.1513 & 86.00 & 0.1863 & 110.00 & 0.2121 \\
\hline 38.50 & 0.0954 & 62.50 & 0.1554 & 86.50 & 0.1871 & 110.50 & 0.2071 \\
\hline 39.00 & 0.0992 & 63.00 & 0.1513 & 87.00 & 0.1863 & 111.00 & 0.2117 \\
\hline 39.50 & 0.1054 & 63.50 & 0.1467 & 87.50 & 0.1858 & 111.50 & 0.2129 \\
\hline 40.00 & 0.1083 & 64.00 & 0.1525 & 88.00 & 0.1933 & 112.00 & 0.2117 \\
\hline 40.50 & 0.1021 & 64.50 & 0.1567 & 88.50 & 0.1858 & 112.50 & 0.2133 \\
\hline 41.00 & 0.1117 & 65.00 & 0.1546 & 89.00 & 0.1950 & 113.00 & 0.2188 \\
\hline 41.50 & 0.1092 & 65.50 & 0.1600 & 89.50 & 0.1933 & 113.50 & 0.2217 \\
\hline 42.00 & 0.1150 & 66.00 & 0.1592 & 90.00 & 0.1946 & 114.00 & 0.2250 \\
\hline 42.50 & 0.1158 & 66.50 & 0.1633 & 90.50 & 0.1983 & 114.50 & 0.2263 \\
\hline 43.00 & 0.1179 & 67.00 & 0.1608 & 91.00 & 0.1979 & 115.00 & 0.2050 \\
\hline 43.50 & 0.1213 & 67.50 & 0.1575 & 91.50 & 0.1992 & 115.50 & 0.2129 \\
\hline 44.00 & 0.1208 & 68.00 & 0.1608 & 92.00 & 0.2013 & 116.00 & 0.2200 \\
\hline 44.50 & 0.1200 & 68.50 & 0.1642 & 92.50 & 0.2063 & 116.50 & 0.2204 \\
\hline 45.00 & 0.1271 & 69.00 & 0.1550 & 93.00 & 0.2046 & 117.00 & 0.2254 \\
\hline 45.50 & 0.1100 & 69.50 & 0.1663 & 93.50 & 0.2058 & 117.50 & 0.2283 \\
\hline 46.00 & 0.1217 & 70.00 & 0.1700 & 94.00 & 0.2092 & 118.00 & 0.2275 \\
\hline 46.50 & 0.1225 & 70.50 & 0.1692 & 94.50 & 0.2096 & 118.50 & 0.2096 \\
\hline 47.00 & 0.1271 & 71.00 & 0.1754 & 95.00 & 0.2133 & 119.00 & 0.2217 \\
\hline 47.50 & 0.1275 & 71.50 & 0.1779 & 95.50 & 0.2088 & 119.50 & 0.2238 \\
\hline 48.00 & 0.1296 & 72.00 & 0.1783 & 96.00 & 0.2079 & 120.00 & 0.2263 \\
\hline 48.50 & 0.1304 & 72.50 & 0.1775 & 96.50 & 0.2108 & 120.50 & 0.2283 \\
\hline
\end{tabular}


Table C11. Thermo-Gravimetric Analysis Data for $\mathrm{K}$ Basin Fuel Sample Run 23 at $150{ }^{\circ} \mathrm{C}$. (Page 2 of 3 )

RUN NUMBER 23 AT $150 \mathrm{C}$

\begin{tabular}{|c|c|c|c|c|c|c|c|}
\hline $\begin{array}{c}\text { TIME } \\
\text { (minutes) }\end{array}$ & \begin{tabular}{|c|}
$\begin{array}{c}\text { wt change } \\
(\mathrm{mg})\end{array}$ \\
\end{tabular} & $\begin{array}{c}\text { TIME } \\
\text { (minutes) }\end{array}$ & $\begin{array}{c}\text { wt change } \\
\text { (mg) }\end{array}$ & $\begin{array}{c}\text { TIME } \\
\text { (minutes) }\end{array}$ & $\begin{array}{c}\text { wt change } \\
\text { (mg) }\end{array}$ & $\begin{array}{c}\text { TIME } \\
\text { (minutes) }\end{array}$ & $\begin{array}{c}\text { wt change } \\
(\mathrm{mg})\end{array}$ \\
\hline 121.00 & 0.2250 & 145.00 & 0.2471 & 169.00 & 0.2846 & 193.00 & 0.2829 \\
\hline 121.50 & 0.2321 & 145.50 & 0.2450 & 169.50 & 0.2767 & 193.50 & 0.2796 \\
\hline 122.00 & 0.2296 & 146.00 & 0.2496 & 170.00 & 0.2771 & 194.00 & 0.2821 \\
\hline 122.50 & 0.2346 & 146.50 & 0.2517 & 170.50 & 0.2783 & 194.50 & 0.2842 \\
\hline 123.00 & 0.2317 & 147.00 & 0.2525 & 171.00 & 0.2846 & 195.00 & 0.2858 \\
\hline 123.50 & 0.2342 & 147.50 & 0.2483 & 171.50 & 0.2763 & 195.50 & 0.2779 \\
\hline 124.00 & 0.2388 & 148.00 & 0.2525 & 172.00 & 0.2825 & 196.00 & 0.2854 \\
\hline 124.50 & 0.2371 & 148.50 & 0.2517 & 172.50 & 0.2792 & 196.50 & 0.2833 \\
\hline 125.00 & 0.2388 & 149.00 & 0.2517 & 173.00 & 0.2842 & 197.00 & 0.2842 \\
\hline 125.50 & 0.2408 & 149.50 & 0.2525 & 173.50 & 0.2742 & 197.50 & 0.2842 \\
\hline 126.00 & 0.2383 & 150.00 & 0.2454 & 174.00 & 0.2775 & 198.00 & 0.2892 \\
\hline 126.50 & 0.2421 & 150.50 & 0.2463 & 174.50 & 0.2846 & 198.50 & 0.2871 \\
\hline 127.00 & 0.2388 & 151.00 & 0.2492 & 175.00 & 0.2825 & 199.00 & 0.2854 \\
\hline 127.50 & 0.2379 & 151.50 & 0.2567 & 175.50 & 0.2833 & 199.50 & 0.2796 \\
\hline 128.00 & 0.2329 & 152.00 & 0.2583 & 176.00 & 0.2825 & 200.00 & 0.2871 \\
\hline 128.50 & 0.2358 & 152.50 & 0.2558 & 176.50 & 0.2829 & 200.50 & 0.2838 \\
\hline 129.00 & 0.2358 & 153.00 & 0.2575 & 177.00 & 0.2846 & 201.00 & 0.2779 \\
\hline 129.50 & 0.2367 & 153.50 & 0.2596 & 177.50 & 0.2904 & 201.50 & 0.2804 \\
\hline 130.00 & 0.2383 & 154.00 & 0.2563 & 178.00 & 0.2738 & 202.00 & 0.2800 \\
\hline 130.50 & 0.2421 & 154.50 & 0.2608 & 178.50 & 0.2825 & 202.50 & 0.2858 \\
\hline 131.00 & 0.2383 & 155.00 & 0.2575 & 179.00 & 0.2792 & 203.00 & 0.2829 \\
\hline 131.50 & 0.2379 & 155.50 & 0.2646 & 179.50 & 0.2854 & 203.50 & 0.2800 \\
\hline 132.00 & 0.2396 & 156.00 & 0.2633 & 180.00 & 0.2804 & 204.00 & 0.2792 \\
\hline 132.50 & 0.2400 & 156.50 & 0.2688 & 180.50 & 0.2813 & 204.50 & 0.2829 \\
\hline 133.00 & 0.2425 & 157.00 & 0.2654 & 181.00 & 0.2733 & 205.00 & 0.2846 \\
\hline 133.50 & 0.2383 & 157.50 & 0.2725 & 181.50 & 0.2783 & 205.50 & 0.2863 \\
\hline 134.00 & 0.2392 & 158.00 & 0.2704 & 182.00 & 0.2817 & 206.00 & 0.2829 \\
\hline 134.50 & 0.2404 & 158.50 & 0.2688 & 182.50 & 0.2821 & 206.50 & 0.2688 \\
\hline 135.00 & 0.2413 & 159.00 & 0.2704 & 183.00 & 0.2808 & 207.00 & 0.2771 \\
\hline 135.50 & 0.2388 & 159.50 & 0.2692 & 183.50 & 0.2825 & 207.50 & 0.2813 \\
\hline 136.00 & 0.2408 & 160.00 & 0.2725 & 184.00 & 0.2842 & 208.00 & 0.2858 \\
\hline 136.50 & 0.2371 & 160.50 & 0.2750 & 184.50 & 0.2829 & 208.50 & 0.2863 \\
\hline 137.00 & 0.2379 & 161.00 & 0.2733 & 185.00 & 0.2871 & 209.00 & 0.2863 \\
\hline 137.50 & 0.2338 & 161.50 & 0.2713 & 185.50 & 0.2825 & 209.50 & 0.2804 \\
\hline 138.00 & 0.2321 & 162.00 & 0.2638 & 186.00 & 0.2850 & 210.00 & 0.2867 \\
\hline 138.50 & 0.2346 & 162.50 & 0.2688 & 186.50 & 0.2842 & 210.50 & 0.2900 \\
\hline 139.00 & 0.2354 & 163.00 & 0.2679 & 187.00 & 0.2783 & 211.00 & 0.2846 \\
\hline 139.50 & 0.2429 & 163.50 & 0.2471 & 187.50 & 0.2854 & 211.50 & 0.2854 \\
\hline 140.00 & 0.2471 & 164.00 & 0.2721 & 188.00 & 0.2821 & 212.00 & 0.2838 \\
\hline 140.50 & 0.2425 & 164.50 & 0.2804 & 188.50 & 0.2829 & 212.50 & 0.2858 \\
\hline 141.00 & 0.2429 & 165.00 & 0.2796 & 189.00 & 0.2883 & 213.00 & 0.2842 \\
\hline 141.50 & & 165.50 & 829 & 189.50 & 0.2804 & 213.50 & 0.2883 \\
\hline 142.00 & 0.2433 & 166.00 & 0.2704 & 190.00 & 0.2854 & 214.00 & 0.2879 \\
\hline 142.50 & 0.2467 & 166.50 & 0.2767 & 190.50 & 0.2842 & 214.50 & 0.2875 \\
\hline 143.00 & 0.2446 & 167.00 & 0.2746 & 191.00 & 0.2821 & 215.00 & 0.2858 \\
\hline 143.50 & 0.2483 & 167.50 & 0.2767 & 191.50 & 0.2850 & 215.50 & 0.2838 \\
\hline 144.00 & 0.2450 & 168.00 & 0.2796 & 192.00 & 0.2854 & 216.00 & 0.2854 \\
\hline 144.50 & 0.2396 & 168.50 & 0.2813 & 192.50 & 0.2846 & 216.50 & 0.2921 \\
\hline
\end{tabular}


Table C11. Thermo-Gravimetric Analysis Data for $K$ Basin Fuel Sample Run 23 at $150^{\circ} \mathrm{C}$. (Page 3 of 3 )

RUN NUMBER 23 AT $150 \mathrm{C}$

\begin{tabular}{|c|c|c|c|c|c|c|c|}
\hline $\begin{array}{c}\text { TIME } \\
\text { (minutes) }\end{array}$ & $\begin{array}{c}\text { wt change } \\
\text { (mg) }\end{array}$ & $\begin{array}{c}\text { TIME } \\
\text { (minutes) }\end{array}$ & $\begin{array}{c}\text { wt change } \\
\text { (mg) }\end{array}$ & $\begin{array}{c}\text { TIME } \\
\text { (minutes) }\end{array}$ & $\begin{array}{c}\text { wt change } \\
(\mathrm{mg})\end{array}$ & $\begin{array}{c}\text { TIME } \\
\text { (minutes) }\end{array}$ & $\begin{array}{l}\text { wt change } \\
\text { (mg) }\end{array}$ \\
\hline 217.00 & 0.2892 & 241.00 & 0.2942 & 265.00 & 0.2858 & 289.00 & 0.2679 \\
\hline 217.50 & 0.2888 & 241.50 & 0.2925 & 265.50 & 0.2875 & 289.50 & 0.2675 \\
\hline 218.00 & 0.2908 & 242.00 & 0.2904 & 266.00 & 0.2933 & 290.00 & 0.2688 \\
\hline 218.50 & 0.2871 & 242.50 & 0.2942 & 266.50 & 0.2896 & 290.50 & 0.2688 \\
\hline 219.00 & 0.2863 & 243.00 & 0.2942 & 267.00 & 0.2933 & 291.00 & 0.2675 \\
\hline 219.50 & 0.2896 & 243.50 & 0.2958 & 267.50 & 0.2875 & 291.50 & 0.2683 \\
\hline 220.00 & 0.2892 & 244.00 & 0.2950 & 268.00 & 0.2913 & 292.00 & 0.2713 \\
\hline 220.50 & 0.2846 & 244.50 & 0.2967 & 268.50 & 0.2863 & 292.50 & 0.2721 \\
\hline 221.00 & 0.2867 & 245.00 & 0.2942 & 269.00 & 0.2850 & 293.00 & 0.2442 \\
\hline 221.50 & 0.2854 & 245.50 & 0.2971 & 269.50 & 0.2779 & 293.50 & 0.2633 \\
\hline 222.00 & 0.2892 & 246.00 & 0.2950 & 270.00 & 0.2717 & 294.00 & 0.2688 \\
\hline 222.50 & 0.2883 & 246.50 & 0.2958 & 270.50 & 0.2825 & 294.50 & 0.2758 \\
\hline 223.00 & 0.2888 & 247.00 & 0.2933 & 271.00 & 0.2792 & 295.00 & 0.2729 \\
\hline 223.50 & 0.2846 & 247.50 & 0.2958 & 271.50 & 0.2913 & 295.50 & 0.2538 \\
\hline 224.00 & 0.2867 & 248.00 & 0.2950 & 272.00 & 0.2863 & 296.00 & 0.2788 \\
\hline 224.50 & 0.2854 & 248.50 & 0.2983 & 272.50 & 0.2792 & 296.50 & 0.2713 \\
\hline 225.00 & 0.2746 & 249.00 & 0.2971 & 273.00 & 0.2829 & 297.00 & 0.2700 \\
\hline 225.50 & 0.2900 & 249.50 & 0.2963 & 273.50 & 0.2883 & 297.50 & 0.2779 \\
\hline 226.00 & 0.2900 & 250.00 & 0.2983 & 274.00 & 0.2842 & 298.00 & 0.2733 \\
\hline 226.50 & 0.2871 & 250.50 & 0.2958 & 274.50 & 0.2821 & 298.50 & 0.2717 \\
\hline 227.00 & 0.2888 & 251.00 & 0.2988 & 275.00 & 0.2775 & 299.00 & 0.2713 \\
\hline 227.50 & 0.2917 & 251.50 & 0.2975 & 275.50 & 0.2842 & 299.50 & 0.2738 \\
\hline 228.00 & 0.2892 & 252.00 & 0.2979 & 276.00 & 0.2838 & 300.00 & 0.2700 \\
\hline 228.50 & 0.2892 & 252.50 & 0.2979 & 276.50 & 0.2808 & 300.50 & 0.2721 \\
\hline 229.00 & 0.2900 & 253.00 & 0.2971 & 277.00 & 0.2533 & 301.00 & 0.2767 \\
\hline 229.50 & 0.2908 & 253.50 & 0.2958 & 277.50 & 0.2758 & 301.50 & 0.2729 \\
\hline 230.00 & 0.2913 & 254.00 & 0.2942 & 278.00 & 0.2654 & 302.00 & 0.2729 \\
\hline 230.50 & 0.2908 & 254.50 & 0.2954 & 278.50 & 0.2792 & 302.50 & 0.2742 \\
\hline 231.00 & 0.2833 & 255.00 & 0.2963 & 279.00 & 0.2625 & 303.00 & 0.2767 \\
\hline 231.50 & 0.2904 & 255.50 & 0.2979 & 279.50 & 0.2738 & 303.50 & 0.2713 \\
\hline 232.00 & 0.2921 & 256.00 & 0.2967 & 280.00 & 0.2692 & 304.00 & 0.2750 \\
\hline 232.50 & 0.2913 & 256.50 & 0.2975 & 280.50 & 0.2779 & 304.50 & 0.2746 \\
\hline 233.00 & 0.2917 & 257.00 & 0.2967 & 281.00 & 0.2763 & 305.00 & 0.2750 \\
\hline 233.50 & 0.2913 & 257.50 & 0.2938 & 281.50 & 0.2804 & 305.50 & 0.2683 \\
\hline 234.00 & 0.2929 & 258.00 & 0.2971 & 282.00 & 0.2788 & 306.00 & 0.2733 \\
\hline 234.50 & 0.2900 & 258.50 & 0.2958 & 282.50 & 0.2683 & 306.50 & 0.2754 \\
\hline 235.00 & 0.2938 & 259.00 & 0.2938 & 283.00 & 0.2754 & 307.00 & 0.2763 \\
\hline 235.50 & 0.2950 & 259.50 & 0.2929 & 283.50 & 0.2767 & 307.50 & 0.2754 \\
\hline 236.00 & 0.2904 & 260.00 & 0.2946 & 284.00 & 0.2583 & 308.00 & 0.2754 \\
\hline 236.50 & 0.2921 & 260.50 & 0.2913 & 284.50 & 0.2658 & 308.50 & 0.2750 \\
\hline 237.00 & 0.2929 & 261.00 & 0.2900 & 285.00 & 0.2575 & 309.00 & 0.2783 \\
\hline 237.50 & 0.2929 & 261.50 & 0.2954 & 285.50 & 0.2604 & 309.50 & 0.2750 \\
\hline 238.00 & 0.2908 & 262.00 & 0.2929 & 286.00 & 0.2617 & & \\
\hline 238.50 & 0.2933 & 262.50 & 0.2896 & 286.50 & 0.2575 & & \\
\hline 239.00 & 0.2921 & 263.00 & 0.2888 & 287.00 & 0.2663 & & \\
\hline 239.50 & 0.2917 & 263.50 & 0.2829 & 287.50 & 0.2696 & & \\
\hline 240.00 & 0.2921 & 264.00 & 0.2792 & 288.00 & 0.2638 & & \\
\hline 240.50 & 0.2904 & 264.50 & 0.2838 & 288.50 & 0.2579 & & \\
\hline
\end{tabular}


Table C12. Thermo-Gravimetric Analysis Data for $K$ Basin Fuel Sample Run 47 at $225^{\circ} \mathrm{C}$. (Page 1 of 2)

\begin{tabular}{|c|c|c|c|c|c|c|c|}
\hline \multicolumn{8}{|c|}{ RUN NUMBER 47 AT $225 \mathrm{C}$} \\
\hline $\begin{array}{c}\text { TIME } \\
\text { (minutes) }\end{array}$ & $\begin{array}{c}\text { wt change } \\
(\mathrm{mg})\end{array}$ & $\begin{array}{c}\text { TIME } \\
\text { (minutes) }\end{array}$ & \begin{tabular}{|c} 
wt change \\
(mg)
\end{tabular} & $\begin{array}{c}\text { TIME } \\
\text { (minutes) }\end{array}$ & \begin{tabular}{|c|}
$\begin{array}{c}\text { wt change } \\
\text { (mg) }\end{array}$ \\
\end{tabular} & $\begin{array}{c}\text { TIME } \\
\text { (minutes) }\end{array}$ & $\begin{array}{c}\text { wt change } \\
\text { (mg) }\end{array}$ \\
\hline 14.00 & -0.1424 & 62.00 & 1.8340 & 110.00 & 2.1497 & 158.00 & 4.1023 \\
\hline 15.00 & -0.0934 & 63.00 & 1.8617 & 111.00 & 2.1670 & 159.00 & 4.1395 \\
\hline 16.00 & -0.0452 & 64.00 & 1.8869 & 112.00 & 2.1849 & 160.00 & 4.1738 \\
\hline 17.00 & 0.0023 & 65.00 & 1.9099 & 113.00 & 2.2036 & 161.00 & 4.2051 \\
\hline 18.00 & 0.0491 & 66.00 & 1.9310 & 114.00 & 2.2231 & 162.00 & 4.2339 \\
\hline 19.00 & 0.0951 & 67.00 & 1.9506 & 115.00 & 2.2432 & 163.00 & 4.2604 \\
\hline 20.00 & 0.1404 & 68.00 & 1.9689 & 116.00 & 2.2639 & 164.00 & 4.2842 \\
\hline 21.00 & 0.1850 & 69.00 & 1.9859 & 117.00 & 2.2852 & 165.00 & 4.3053 \\
\hline 22.00 & 0.2289 & 70.00 & 2.0019 & 118.00 & 2.3070 & 166.00 & 4.3244 \\
\hline 23.00 & 0.2723 & 71.00 & 2.0166 & 119.00 & 2.3286 & 167.00 & 4.3421 \\
\hline 24.00 & 0.3151 & 72.00 & 2.0299 & 120.00 & 2.3505 & 168.00 & 4.3591 \\
\hline 25.00 & 0.3574 & 73.00 & 2.0414 & 121.00 & 2.3729 & 169.00 & 4.3757 \\
\hline 26.00 & 0.3993 & 74.00 & 2.0510 & 122.00 & 2.3965 & 170.00 & 4.3927 \\
\hline 27.00 & 0.4407 & 75.00 & 2,0584 & 123.00 & 2.4218 & 171.00 & 4.4109 \\
\hline 28.00 & 0.4817 & 76.00 & 2.0630 & 124.00 & 2.4491 & 172.00 & 4.4312 \\
\hline 29.00 & 0.5223 & 77.00 & 2.0645 & 125.00 & 2.4786 & 173.00 & 4.4537 \\
\hline 30.00 & 0.5625 & 78.00 & 2.0631 & 126.00 & 2.5097 & 174.00 & 4.4786 \\
\hline 31.00 & 0.6024 & 79.00 & 2.0586 & 127.00 & 2.5421 & 175.00 & 4.5058 \\
\hline 32.00 & 0.6420 & 80.00 & 2.0508 & 128.00 & 2.5756 & 176.00 & 4.5345 \\
\hline 33.00 & 0.6813 & 81.00 & 2.0393 & 129.00 & 2.6099 & 177.00 & 4.5644 \\
\hline 34.00 & 0.7205 & 82.00 & 2.0237 & 130.00 & 2.6445 & 178.00 & 4.5955 \\
\hline 35.00 & 0.7595 & 83.00 & 2.0036 & 131.00 & 2.6794 & 179.00 & 4.6272 \\
\hline 36.00 & 0.7983 & 84.00 & 1.9791 & 132.00 & 2.7143 & 180.00 & 4.6590 \\
\hline 37.00 & 0.8368 & 85.00 & 1.9513 & 133.00 & 2.7491 & 181.00 & 4.6905 \\
\hline 38.00 & 0.8750 & 86.00 & 1.9214 & 134.00 & 2.7835 & 182.00 & 4.7216 \\
\hline 39.00 & 0.9127 & 87.00 & 1.8907 & 135.00 & 2.8176 & 183.00 & 4.7526 \\
\hline 40.00 & 0.9494 & 88.00 & 1.8605 & 136.00 & 2.8519 & 184.00 & 4.7839 \\
\hline 41.00 & 0.9844 & 89.00 & 1.8325 & 137.00 & 2.8876 & 185.00 & 4.8156 \\
\hline 42.00 & 1.0162 & 90.00 & 1.8086 & 138.00 & 2.9261 & 186.00 & 4.8485 \\
\hline 43.00 & 1.0432 & 91.00 & 1.7903 & 139.00 & 2.9687 & 187.00 & 4.8825 \\
\hline 44.00 & 1.0733 & 92.00 & 1.7787 & 140.00 & 3.0164 & 188.00 & 4.9180 \\
\hline 45.00 & 1.1070 & 93.00 & 1.7738 & 141.00 & 3.0690 & 189.00 & 4.9553 \\
\hline 46.00 & 1.1445 & 94.00 & 1.7758 & 142.00 & 3.1261 & 190.00 & 4.9950 \\
\hline 47.00 & 1.1859 & 95.00 & 1.7844 & 143.00 & 3.1874 & 191.00 & 5.0380 \\
\hline 48.00 & 1.2310 & 96.00 & 1.7992 & 144.00 & 3.2523 & 192.00 & 5.0849 \\
\hline 49.00 & 1.2795 & 97.00 & 1.8196 & 145.00 & 3.3201 & 193.00 & 5.1361 \\
\hline 50.00 & 1.3302 & 98.00 & 1.8450 & 146.00 & 3.3897 & 194.00 & 5.1911 \\
\hline 51.00 & 1.3821 & 99.00 & 1.8739 & 147.00 & 3.4600 & 195.00 & 5.2491 \\
\hline 52.00 & 1.4338 & 100.00 & 1.9051 & 148.00 & 3.5304 & 196.00 & 5.3095 \\
\hline 53.00 & 1.4842 & 101.00 & 1.9371 & 149.00 & 3.6001 & 197.00 & 5.3719 \\
\hline 54.00 & 1.5325 & 102.00 & 1.9686 & 150.00 & 3.6687 & 198.00 & 5,4366 \\
\hline 55.00 & 1.5781 & 103.00 & 1.9984 & 151.00 & 3.7355 & 199.00 & 5.5034 \\
\hline 56.00 & 1.6209 & 104.00 & 2.0259 & 152.00 & 3.7995 & 200.00 & 5.5725 \\
\hline 57.00 & 1.6610 & 105.00 & 2.0512 & 153.00 & 3.8601 & 201.00 & 5.6439 \\
\hline 58.00 & 1.6992 & 106.00 & 2.0743 & 154.00 & 3.9166 & 202.00 & 5.7173 \\
\hline 59.00 & 1.7359 & 107.00 & 2.0952 & 155.00 & 3.9688 & 203.00 & 5.7923 \\
\hline 60.00 & 1.7708 & 108.00 & 2.1144 & 156.00 & 4.0171 & 204.00 & 5.8685 \\
\hline 61.00 & 1.8036 & 109.00 & 2.1323 & 157.00 & 4.0616 & 205.00 & 5.9454 \\
\hline
\end{tabular}


Table C12. Thermo-Gravimetric Analysis Data for K Basin Fuet Sample Run 47 at $225^{\circ} \mathrm{C}$. (Page 2 of 2)

\begin{tabular}{|c|c|c|c|c|c|}
\hline \multicolumn{6}{|c|}{ RUN NUMBER 47 AT 225 C } \\
\hline $\begin{array}{c}\text { TIME } \\
\text { (minutes) }\end{array}$ & \begin{tabular}{|c} 
wt change \\
(mg)
\end{tabular} & $\begin{array}{c}\text { TIME } \\
\text { (minutes) }\end{array}$ & $\begin{array}{c}\text { wt change } \\
\text { (mg) }\end{array}$ & $\begin{array}{c}\text { TIME } \\
\text { (minutes) }\end{array}$ & $\begin{array}{c}\text { wt change } \\
(\mathrm{mg})\end{array}$ \\
\hline 206.00 & 6.0220 & 254.00 & 10.8621 & 302.00 & 14.2225 \\
\hline 207.00 & 6.0974 & 255.00 & 10.9338 & 303.00 & 14.3200 \\
\hline 208.00 & 6.1709 & 256.00 & 10.9992 & 304.00 & 14.4174 \\
\hline 209.00 & 6.2425 & 257.00 & 11.0584 & 305.00 & 14.5144 \\
\hline 210.00 & 6.3120 & 258.00 & 11.1117 & 305.00 & 14.6110 \\
\hline 211.00 & 6.3800 & 259.00 & 11.1598 & 307.00 & 14.7072 \\
\hline 212.00 & 6.4473 & 260.00 & 11.2031 & 308.00 & 14.8028 \\
\hline 213.00 & 6.5155 & 261.00 & 11.2417 & 309.00 & $14 . \overline{8978}$ \\
\hline 214.00 & 6.5862 & 262.00 & 11.2764 & 310.00 & 14.9923 \\
\hline 215.00 & 6.6602 & 263.00 & 11.3085 & 311.00 & 15.0863 \\
\hline 216.00 & 6.7382 & 264.00 & 11.3393 & 312.00 & 15.1799 \\
\hline 217.00 & 6.8202 & 265.00 & 11.3702 & & \\
\hline 218.00 & 6.9064 & 266.00 & 11.4028 & & \\
\hline 219.00 & 6.9969 & 267.00 & 11.4385 & & \\
\hline 220.00 & 7.0922 & 268.00 & 11.4776 & & \\
\hline 221.00 & 7.1920 & 269.00 & 11.5203 & & \\
\hline 222.00 & 7.2950 & 270.00 & 11.5661 & & \\
\hline 223.00 & 7.4004 & 271.00 & 11.6157 & & \\
\hline 224.00 & 7.5074 & 272.00 & 11.6698 & & \\
\hline 225.00 & 7.6161 & 273.00 & 11.7296 & & \\
\hline 226.00 & 7.7262 & 274.00 & 11.7954 & & \\
\hline 227.00 & 7.8375 & 275.00 & 11.8663 & & \\
\hline 228.00 & 7.9498 & 276.00 & 11.9409 & & \\
\hline 229.00 & 8.0632 & 277.00 & 12.0184 & & \\
\hline 230.00 & 8.1783 & 278.00 & 12.0984 & & \\
\hline 231.00 & 8.2946 & 279.00 & 12.1801 & & \\
\hline 232.00 & 8.4120 & 280.00 & 12.2623 & & \\
\hline 233.00 & 8.5298 & 281.00 & 12.3442 & & \\
\hline 234.00 & 8.6475 & 282.00 & 12.4251 & & \\
\hline 235.00 & 8.7649 & 283.00 & 12.5048 & & \\
\hline 236.00 & 8.8823 & 284.00 & 12.5952 & & \\
\hline 237.00 & 9.0004 & 285.00 & 12.6799 & & \\
\hline 238.00 & 9.1195 & 286.00 & 12.7626 & & \\
\hline 239.00 & 9.2395 & 287.00 & 12.8449 & & \\
\hline 240.00 & 9.3600 & 288.00 & 12.9280 & & \\
\hline 241.00 & 9.4807 & 289.00 & 13.0121 & & \\
\hline 242.00 & 9.6012 & 290.00 & 13.0976 & & \\
\hline 243.00 & 9.7214 & 291.00 & 13.1846 & & \\
\hline 244.00 & 9.8408 & 292.00 & 13.2733 & & \\
\hline 245.00 & 9.9590 & 293.00 & 13.3634 & & \\
\hline 246.00 & 10.0753 & 294.00 & 13.4551 & & \\
\hline 247.00 & 10.1891 & 295.00 & 13.5480 & & \\
\hline 248.00 & 10.2996 & 296.00 & 13.6422 & & \\
\hline 249.00 & 10.4061 & 297.00 & 13.7373 & & \\
\hline 250.00 & 10.5081 & 298.00 & 13.8333 & & \\
\hline 251.00 & 10.6056 & 299.00 & 13.9300 & & \\
\hline 252.00 & 10.6978 & 300.00 & 14.0272 & & \\
\hline 253.00 & 10.7835 & 301.00 & 14.1248 & & \\
\hline
\end{tabular}


Table C13. Thermo-Gravimetric Analysis Data for $K$ Bas in Fuel Sample Run 48 at $275^{\circ} \mathrm{C}$. (Page 1 of 2 )

\begin{tabular}{|c|c|c|c|c|c|c|c|}
\hline \multicolumn{8}{|c|}{ RUN NUMBER 48 AT $275 \mathrm{C}$} \\
\hline $\begin{array}{c}\text { TIME } \\
\text { (minutes) }\end{array}$ & $\begin{array}{c}\text { wt change } \\
\text { (mg) }\end{array}$ & $\begin{array}{c}\text { TIME } \\
\text { (minutes) }\end{array}$ & $\begin{array}{c}\text { wt change } \\
\text { (mg) }\end{array}$ & $\begin{array}{c}\text { TIME } \\
\text { (minutes) }\end{array}$ & $\begin{array}{c}\text { wt change } \\
\text { (mg) }\end{array}$ & $\begin{array}{c}\text { TiME } \\
\text { (minutes) }\end{array}$ & $\begin{array}{c}\text { wt change } \\
\text { (mg) }\end{array}$ \\
\hline 14.00 & -0.1424 & 62.00 & 1.8340 & 110.00 & 2.1497 & 158.00 & 4.1023 \\
\hline 15.00 & -0.0934 & 63.00 & 1.8617 & 111.00 & 2.1670 & 159.00 & 4.1395 \\
\hline 16.00 & -0.0452 & 64.00 & 1.8869 & 112.00 & 2.1849 & 160.00 & 4.1738 \\
\hline 17.00 & 0.0023 & 65.00 & 1.9099 & 113.00 & 2.2036 & 161.00 & 4.2051 \\
\hline 18.00 & 0.0491 & 66.00 & 1.9310 & 114.00 & 2.2231 & 162.00 & 4.2339 \\
\hline 19.00 & 0.0951 & 67.00 & 1.9506 & 115.00 & 2.2432 & 163.00 & 4.2604 \\
\hline 20.00 & 0.1404 & 68.00 & 1.9689 & 116.00 & 2.2639 & 164.00 & 4.2842 \\
\hline 21.00 & 0.1850 & 69.00 & 1.9859 & 117.00 & 2.2852 & 165.00 & 4.3053 \\
\hline 22.00 & 0.2289 & 70.00 & 2.0019 & 118.00 & 2.3070 & 166.00 & 4.3244 \\
\hline 23.00 & 0.2723 & 71.00 & 2.0166 & 119.00 & 2.3286 & 167.00 & 4.3421 \\
\hline 24.00 & 0.3151 & 72.00 & 2.0299 & 120.00 & 2.3505 & 168.00 & 4.3591 \\
\hline 25.00 & 0.3574 & 73.00 & 2.0414 & 121.00 & 2.3729 & 169.00 & 4.3757 \\
\hline 26.00 & 0.3993 & 74.00 & 2.0510 & 122.00 & 2.3965 & 170.00 & 4.3927 \\
\hline 27.00 & 0.4407 & 75.00 & 2.0584 & 123.00 & 2.4218 & 171.00 & 4.4109 \\
\hline 28.00 & 0.4817 & 76.00 & 2.0630 & 124.00 & 2.4491 & 172.00 & 4.4312 \\
\hline 29.00 & 0.5223 & 77.00 & 2.0645 & 125.00 & 2.4786 & 173.00 & 4.4537 \\
\hline 30.00 & 0.5625 & 78.00 & 2.0631 & 126.00 & 2.5097 & 174.00 & 4.4786 \\
\hline 31.00 & 0.6024 & 79.00 & 2.0586 & 127.00 & 2.5421 & 175.00 & 4.5058 \\
\hline 32.00 & 0.6420 & 80.00 & 2.0508 & 128.00 & 2.5756 & 176.00 & 4.5345 \\
\hline 33.00 & 0.6813 & 81.00 & 2.0393 & 129.00 & 2.6099 & 177.00 & 4.5644 \\
\hline 34.00 & 0.7205 & 82.00 & 2.0237 & 130.00 & 2.6445 & 178.00 & 4.5955 \\
\hline 35.00 & 0.7595 & 83.00 & 2.0036 & 131.00 & 2.6794 & 179.00 & 4.6272 \\
\hline 36.00 & 0.7983 & 84.00 & 1.9791 & 132.00 & 2.7143 & 180.00 & 4.6590 \\
\hline 37.00 & 0.8368 & 85.00 & 1.9513 & 133.00 & 2.7491 & 181.00 & 4.6905 \\
\hline 38.00 & 0.8750 & 86.00 & 1.9214 & 134.00 & 2.7835 & 182.00 & 4.7216 \\
\hline 39.00 & 0.9127 & 87.00 & 1.8907 & 135.00 & 2.8176 & 183.00 & 4.7526 \\
\hline 40.00 & 0.9494 & 88.00 & 1.8605 & 136.00 & 2.8519 & 184.00 & 4.7839 \\
\hline 41.00 & 0.9844 & 89.00 & 1.8325 & 137.00 & 2.8876 & 185.00 & 4.8156 \\
\hline 42.00 & 1.0162 & 90.00 & 1.8086 & 138.00 & 2.9261 & 186.00 & 4.8485 \\
\hline 43.00 & 1.0432 & 91.00 & 1.7903 & 139.00 & 2.9687 & 187.00 & 4.8825 \\
\hline 44.00 & 1.0733 & 92.00 & 1.7787 & 140.00 & 3.0164 & 188.00 & 4.9180 \\
\hline 45.00 & 1.1070 & 93.00 & 1.7738 & 141.00 & 3.0690 & 189.00 & 4.9553 \\
\hline 46.00 & 1.1445 & 94.00 & 1.7758 & 142.00 & 3.1261 & 190.00 & 4.9950 \\
\hline 47.00 & 1.1859 & 95.00 & 1.7844 & 143.00 & 3.1874 & 191.00 & 5.0380 \\
\hline 48.00 & 1.2310 & 96.00 & 1.7992 & 144.00 & 3.2523 & 192.00 & 5.0849 \\
\hline 49.00 & 1.2795 & 97.00 & 1.8196 & 145.00 & 3.3201 & 193.00 & 5.1361 \\
\hline 50.00 & 1.3302 & 98.00 & 1.8450 & 146.00 & 3.3897 & 194.00 & 5.1911 \\
\hline 51.00 & 1.3821 & 99.00 & 1.8739 & 147.00 & 3.4600 & 195.00 & 5.2491 \\
\hline 52.00 & 1.4338 & 100.00 & 1.9051 & 148.00 & 3.5304 & 196.00 & 5.3095 \\
\hline 53.00 & 1.4842 & 101.00 & 1.9371 & 149.00 & 3.6001 & 197.00 & 5.3719 \\
\hline 54.00 & 1.5325 & 102.00 & 1.9686 & 150.00 & 3.6687 & 198.00 & 5.4366 \\
\hline 55.00 & 1.5781 & 103.00 & 1.9984 & 151.00 & 3.7355 & 199.00 & 5.5034 \\
\hline 56.00 & 1.6209 & 104.00 & 2.0259 & 152.00 & 3.7995 & 200.00 & 5.5725 \\
\hline 57.00 & 1.6610 & 105.00 & 2.0512 & 153.00 & 3.8601 & 201.00 & 5.6439 \\
\hline 58.00 & 1.6992 & 106.00 & 2.0743 & 154.00 & 3.9166 & 202.00 & 5.7173 \\
\hline 59.00 & 1.7359 & 107.00 & 2.0952 & 155.00 & 3.9688 & 203.00 & 5.7923 \\
\hline 60.00 & 1.7708 & 108.00 & 2.1144 & 156.00 & 4.0171 & 204.00 & 5.8685 \\
\hline 61.00 & 1.8036 & 109.00 & 2.1323 & 157.00 & 4.0616 & 205.00 & 5.9454 \\
\hline
\end{tabular}


Table C13. Thermo-Gravimetric Analysis Data for K Basin Fuel Sample Run 48 at $275^{\circ} \mathrm{C}$. (Page 2 of 2)

\begin{tabular}{|c|c|c|c|c|c|}
\hline \multicolumn{6}{|c|}{ RUN NUMBER 48 AT 275 C (cont.) } \\
\hline $\begin{array}{c}\text { TIME } \\
\text { (minutes) }\end{array}$ & $\begin{array}{c}\text { wt change } \\
\text { (mg) }\end{array}$ & $\begin{array}{c}\text { TIME } \\
\text { (minutes) }\end{array}$ & $\begin{array}{c}\text { wt change } \\
\text { (mg) }\end{array}$ & $\begin{array}{c}\text { TIME } \\
\text { (minutes) }\end{array}$ & $\begin{array}{l}\text { wt change } \\
\text { (mg) }\end{array}$ \\
\hline 206.00 & 6.0220 & 254.00 & 10.8621 & 302.00 & 14.2225 \\
\hline 207.00 & 6.0974 & 255.00 & 10.9338 & 303.00 & 14.3200 \\
\hline 208.00 & 6.1709 & 256.00 & 10.9992 & 304.00 & 14.4174 \\
\hline 209.00 & 6.2425 & 257.00 & 11.0584 & 305.00 & 14.5144 \\
\hline 210.00 & 6.3120 & 258.00 & 11.1117 & 306.00 & 14.6110 \\
\hline 211.00 & 6.3800 & 259.00 & 11.1598 & 307.00 & 14.7072 \\
\hline 212.00 & 6.4473 & 260.00 & 11.2031 & 308.00 & 14.8028 \\
\hline 213.00 & 6.5155 & 261.00 & 11.2417 & 309.00 & 14.8978 \\
\hline 214.00 & 6.5862 & 262.00 & 11.2764 & 310.00 & 14.9923 \\
\hline 215.00 & 6.6602 & 263.00 & 11.3085 & 311.00 & 15.0863 \\
\hline 216.00 & 6.7382 & 264.00 & 11.3393 & 312.00 & 15.1799 \\
\hline 217.00 & 6.8202 & 265.00 & 11.3702 & & \\
\hline 218.00 & 6.9064 & 266.00 & $\uparrow 1.4028$ & & \\
\hline 219.00 & 6.9969 & 267.00 & 11.4385 & & \\
\hline 220.00 & 7.0922 & 268.00 & 11.4776 & & \\
\hline 221.00 & 7.1920 & 269.00 & 11.5203 & & \\
\hline 222.00 & 7.2950 & 270.00 & 11.5661 & & \\
\hline 223.00 & 7.4004 & 271.00 & 11.6157 & & \\
\hline 224.00 & 7.5074 & 272.00 & 11.6698 & & \\
\hline 225.00 & 7.6161 & 273.00 & 11.7296 & & \\
\hline 226.00 & 7.7262 & 274.00 & 11.7954 & & \\
\hline 227.00 & 7.8375 & 275.00 & 11.8663 & & \\
\hline 228.00 & 7.9498 & 276.00 & 11.9409 & & \\
\hline 229.00 & 8.0632 & 277.00 & 12.0184 & & \\
\hline 230.00 & 8.1783 & 278.00 & 12.0984 & & \\
\hline 231.00 & 8.2946 & 279.00 & 12.1801 & & \\
\hline 232.00 & 8.4120 & 280.00 & 12.2623 & & \\
\hline 233.00 & 8.5298 & 281.00 & 12.3442 & & \\
\hline 234.00 & 8.6475 & 282.00 & 12.4251 & & \\
\hline 235.00 & 8.7649 & 283.00 & 12.5048 & & \\
\hline 236.00 & 8.8823 & 284.00 & 12.5952 & & \\
\hline 237.00 & 9.0004 & 285.00 & 12.6799 & & \\
\hline 238.00 & 9.1195 & 286.00 & 12.7626 & & \\
\hline 239.00 & 9.2395 & 287.00 & 12.8449 & & \\
\hline 240.00 & 9.3600 & 288.00 & 12.9280 & & \\
\hline 241.00 & 9.4807 & 289.00 & 13.0121 & & \\
\hline 242.00 & 9.6012 & 290.00 & 13.0976 & & \\
\hline 243.00 & 9.7214 & 291.00 & 13.1846 & & \\
\hline 244.00 & 9.8408 & 292.00 & 13.2733 & & \\
\hline 245.00 & 9.9590 & 293.00 & 13.3634 & & \\
\hline 246.00 & 10.0753 & 294.00 & 3.4551 & & \\
\hline 247.00 & 10.1891 & 295.00 & 13.5480 & & \\
\hline 248.00 & 10.2996 & 296.00 & 13.6422 & & \\
\hline 249.00 & 10.4061 & 297.00 & 13.7373 & & \\
\hline 250.00 & 10.5081 & 298.00 & 13.8333 & & \\
\hline 251.00 & 10.6056 & 299.00 & 13.9300 & & \\
\hline 252.00 & 10.6978 & 300.00 & 14.0272 & & \\
\hline 253.00 & 10.7835 & 301.00 & 14.1248 & & \\
\hline
\end{tabular}


Table C14. Thermo-Gravimetric Anaiysis Data for K Basin Fuel Sample Run $49 \mathrm{a}$ at $90^{\circ} \mathrm{C}$. (Page 1 of 6 )

\begin{tabular}{|c|c|c|c|c|c|c|c|}
\hline \multicolumn{8}{|c|}{ RUN NUMBER 49a AT $90 \mathrm{C}$} \\
\hline $\begin{array}{l}\text { TTME } \\
\text { (minutes) }\end{array}$ & $\begin{array}{c}\text { wt change } \\
\text { (mg) }\end{array}$ & $\begin{array}{l}\text { TIME } \\
\text { (minutes) }\end{array}$ & \begin{tabular}{|c|} 
wt change \\
(mg)
\end{tabular} & $\begin{array}{l}\text { TIME } \\
\text { (minutes) }\end{array}$ & $\begin{array}{c}\text { wt change } \\
\text { (mg) }\end{array}$ & $\begin{array}{l}\text { TIME } \\
\text { (minutes) }\end{array}$ & $\begin{array}{c}\text { wt change } \\
\text { (mg) }\end{array}$ \\
\hline 10.00 & 0.313 & 60.00 & 0.758 & 110,00 & 0.900 & 160.00 & 1.025 \\
\hline 11.00 & 0.329 & 61.00 & 0.771. & 111.00 & 0.908 & 161.00 & 1.025 \\
\hline 12.00 & 0.342 & 62.00 & 0.783 & 112.00 & 0.908 & 162.00 & 1.033 \\
\hline 13.00 & 0.363 & 63.00 & 0.779 & 113.00 & 0.896 & 163.00 & 1.025 \\
\hline 14.00 & 0.363 & 64.00 & 0.783 & 114.00 & 0.900 & 164.00 & 1.029 \\
\hline 15.00 & 0.371 & 65.00 & 0.792 & 115.00 & 0.900 & 165.00 & 1.038 \\
\hline 16.00 & 0.375 & 66.00 & 0.792 & 116.00 & 0.900 & 166.00 & 1.038 \\
\hline 17.00 & 0.388 & 67.00 & 0.767 & 117.00 & 0.904 & 167.00 & 1.042 \\
\hline 18.00 & 0.392 & 68.00 & 0.779 & 118.00 & 0.896 & 168.00 & 1.038 \\
\hline 19.00 & 0.396 & 69.00 & 0.792 & 119.00 & 0.913 & 169.00 & 1.042 \\
\hline 20.00 & 0.388 & 70.00 & 0.783 & 120.00 & 0.913 & 170.00 & 1.038 \\
\hline 21.00 & 0.425 & 71.00 & 0.767 & 121.00 & 0.929 & 171.00 & 1.038 \\
\hline 22.00 & 0.438 & 72.00 & 0.783 & 122.00 & 0.925 & 172.00 & 1.042 \\
\hline 23.00 & 0.463 & 73.00 & 0.800 & 123.00 & 0.933 & 173.00 & 1.038 \\
\hline 24.00 & 0.483 & 74.00 & 0.800 & 124.00 & 0.925 & 174.00 & 1.054 \\
\hline 25.00 & 0.500 & 75.00 & 0.788 & 125.00 & 0.929 & 175.00 & 1.063 \\
\hline 26.00 & 0.517 & 76.00 & 0.783 & 126.00 & 0.933 & 176.00 & 1.067 \\
\hline 27.00 & 0.521 & 77.00 & 0.796 & 127.00 & 0.925 & 177.00 & 1.063 \\
\hline 28.00 & 0.529 & 78.00 & 0.808 & 128.00 & 0.925 & 178.00 & 1.067 \\
\hline 29,00 & 0.533 & 79.00 & 0.796 & 129.00 & 0.929 & 179.00 & 1.075 \\
\hline 30.00 & 0.538 & 80.00 & 0.808 & 130.00 & 0.929 & 180.00 & 1.075 \\
\hline 31.00 & 0.550 & 81.00 & 0.813 & 131.00 & 0.921 & 181.00 & 1.075 \\
\hline 32.00 & 0.554 & 82.00 & 0.817 & 132.00 & 0.900 & 182.00 & 1.088 \\
\hline 33.00 & 0.575 & 83.00 & 0.821 & 133.00 & 0.908 & 183.00 & 1.088 \\
\hline 34.00 & 0.592 & 84.00 & 0.821 & 134.00 & 0.925 & 184.00 & 1.088 \\
\hline 35.00 & 0.604 & 85.00 & 0.825 & 135.00 & 0.929 & 185.00 & 1.088 \\
\hline 36.00 & 0.608 & 86.00 & 0.817 & 136.00 & 0.925 & 186.00 & 1.092 \\
\hline 37.00 & 0.613 & 87.00 & 0.800 & 137.00 & 0.942 & 187.00 & 1.088 \\
\hline 38.00 & 0.625 & 88.00 & 0.792 & 138.00 & 0.950 & 188.00 & 1.088 \\
\hline 39.00 & 0.637 & 89.00 & 0.800 & 139.00 & 0.950 & 189.00 & 1.100 \\
\hline 40.00 & 0.637 & 90.00 & 0.800 & 140.00 & 0.967 & 190.00 & 1.108 \\
\hline 41.00 & 0.658 & 91.00 & 0.808 & 141.00 & 0.967 & 191.00 & 1.100 \\
\hline 42.00 & 0.650 & 92.00 & 0.825 & 142.00 & 0.954 & 192.00 & 1.104 \\
\hline 43.00 & 0.671 & 93.00 & 0.825 & 143.00 & 0.967 & 193.00 & 1.104 \\
\hline 44.00 & 0.675 & 94.00 & 0.829 & 144.00 & 0.967 & 194.00 & 1.113 \\
\hline 45.00 & 0.679 & 95.00 & 0.833 & 145.00 & 0.988 & 195.00 & 1.108 \\
\hline 46.00 & 0.679 & 96.00 & 0.838 & 146.00 & 1.000 & 196.00 & 1.104 \\
\hline 47.00 & 0.692 & 97.00 & 0.850 & 147.00 & 1.008 & 197.00 & 1.117 \\
\hline 48.00 & 0.704 & 98.00 & 0.842 & 148.00 & 0.992 & 198.00 & 1.125 \\
\hline 49.00 & 0.713 & 99.00 & 0.842 & 149.00 & 0.988 & 199.00 & 1.121 \\
\hline 50.00 & 0.717 & 100.00 & 0.858 & 150.00 & 0.988 & 200.00 & 1.125 \\
\hline 51.00 & 0.725 & 101.00 & 0.863 & 151.00 & 1.008 & 201.00 & 1.121 \\
\hline 52.00 & 0.725 & 102.00 & 0.867 & 152.00 & 1.013 & 202.00 & 1.133 \\
\hline 53.00 & 0.721 & 103.00 & 0.875 & 153.00 & 1.025 & 203.00 & 1.125 \\
\hline 54.00 & 0.725 & 104.00 & 0.875 & 154.00 & 1.017 & 204.00 & 1.121 \\
\hline 55.00 & 0.738 & 105.00 & 0.883 & 155.00 & 1.021 & 205.00 & 1.125 \\
\hline 56.00 & 0.742 & 106.00 & 0.888 & 156.00 & 1.017 & 206.00 & 1.108 \\
\hline 57.00 & 0.750 & 107.00 & 0.888 & 157.00 & 1.017 & 207.00 & 1.108 \\
\hline 58.00 & 0.758 & 108.00 & 0.888 & 158.00 & 1.025 & 208.00 & 1.108 \\
\hline 59.00 & 0.754 & 109.00 & 0.892 & 159.00 & 1.029 & 200.00 & 1.113 \\
\hline
\end{tabular}


Table C14. Thermo-Gravimetric Analysis Data for $\mathrm{K}$ Basin Fue] Sample Run $49 \mathrm{a}$ at $90^{\circ} \mathrm{C}$. (Page 2 of 6 )

\begin{tabular}{|c|c|c|c|c|c|c|c|}
\hline \multicolumn{8}{|c|}{ RUN NUMBER 49a AT $90 \mathrm{C}$ (cont.) } \\
\hline $\begin{array}{l}\text { TIME } \\
\text { (minutes) }\end{array}$ & \begin{tabular}{|c|}
$\begin{array}{c}\text { wt change } \\
(\mathrm{mg})\end{array}$ \\
\end{tabular} & $\begin{array}{l}\text { TIME } \\
\text { (minutes) }\end{array}$ & $\begin{array}{c}\text { wt change } \\
\text { (mg) }\end{array}$ & $\begin{array}{l}\text { TIME } \\
\text { (minutes) }\end{array}$ & $\begin{array}{c}\text { wt change } \\
(\mathrm{mg})\end{array}$ & $\begin{array}{l}\text { TIME } \\
\text { (minutes) }\end{array}$ & $\begin{array}{c}\text { wt change } \\
\text { (mg) }\end{array}$ \\
\hline 210.00 & 1.108 & 260.00 & 1.150 & 310.00 & 1.213 & 360.00 & 1.238 \\
\hline 211.00 & 1.108 & 261.00 & 1.154 & 311.00 & 1.213 & 361.00 & 1.229 \\
\hline 212.00 & 1.108 & 262.00 & 1.158 & 312.00 & 1.213 & 362.00 & 1.238 \\
\hline 213.00 & 1.117 & 263.00 & 1.154 & 313.00 & 1.217 & 363.00 & 1.246 \\
\hline 214.00 & 1.125 & 264.00 & 1.150 & 314.00 & 1.225 & 364.00 & 1.250 \\
\hline 215.00 & 1.138 & 265.00 & 1.150 & 315.00 & 1.225 & 365.00 & 1.250 \\
\hline 216.00 & 1.142 & 266.00 & 1.150 & 316.00 & 1.225 & 366.00 & 1.238 \\
\hline 217.00 & 1.142 & 267.00 & 1.150 & 317.00 & 1.221 & 367.00 & 1.238 \\
\hline 218.00 & 7.138 & 268.00 & 1.163 & 318.00 & 1.225 & 368.00 & 1.233 \\
\hline 21900 & 1.133 & 269.00 & 1.171 & 319.00 & 1.225 & 369.00 & 1.225 \\
\hline 220.00 & 1.125 & 270.00 & 1.167 & 320.00 & 1.225 & 370.00 & 1.233 \\
\hline 221.00 & 1.121 & 271.00 & 1.175 & 321.00 & 1.225 & 371.00 & 1.233 \\
\hline 222.00 & 1.125 & 272.00 & 1.175 & 322.00 & 1.233 & 372.00 & 1.233 \\
\hline 223.00 & 1.125 & 273.00 & 1.171 & 323.00 & 1.229 & 373.00 & 1.238 \\
\hline 224.00 & 1.125 & 274.00 & 1.167 & 324.00 & 1.225 & 374.00 & 1.238 \\
\hline 225.00 & 1.125 & 275.00 & 1.163 & 325.00 & 1.229 & 375.00 & 1.233 \\
\hline 226.00 & 1.129 & 276.00 & 1.158 & 326.00 & 1.233 & 376.00 & 1.225 \\
\hline 227.00 & 1.125 & 277.00 & 1.167 & 327.00 & 1.238 & 377.00 & 1.233 \\
\hline 228.00 & 1.142 & 278.00 & 1.175 & 328.00 & 1.225 & 378.00 & 1.238 \\
\hline 229.00 & 1.150 & 279.00 & 1.175 & 329.00 & 1.225 & 379.00 & 1.238 \\
\hline 230.00 & 1.146 & 280.00 & 1.175 & 330.00 & 1.225 & 380.00 & 1.233 \\
\hline 231.00 & 1.150 & 281.00 & 1.175 & 331.00 & 1.229 & 381.00 & 1.238 \\
\hline 232.00 & 1.146 & 282.00 & 1.171 & 332.00 & 1.238 & 382.00 & 1.233 \\
\hline 233.00 & 1.150 & 283.00 & 1.167 & 333.00 & 1.229 & 383.00 & 1.238 \\
\hline 234.00 & 1.150 & 284.00 & 1.167 & 334.00 & 1.242 & 384.00 & 1.242 \\
\hline 235.00 & 1.133 & 285.00 & 1.175 & 335.00 & 1.254 & 385.00 & 1.238 \\
\hline 236.00 & 1.138 & 286.00 & 1.175 & 336.00 & 1.250 & 386.00 & 1.238 \\
\hline 237.00 & 1.146 & 287.00 & 1.188 & 337.00 & 1.250 & 387.00 & 1.233 \\
\hline 238.00 & 1.158 & 288.00 & 1.188 & 338.00 & 1.250 & 388.00 & 1.229 \\
\hline 239.00 & 1.150 & 289.00 & 1.183 & 339.00 & 1.250 & 389.00 & 1.225 \\
\hline 240.00 & 1.138 & 290.00 & 1.192 & 340.00 & 1.258 & 390.00 & 1.225 \\
\hline 241.00 & 1.138 & 291.00 & 1.200 & 341.00 & 1.258 & 391.00 & 1.225 \\
\hline 242.00 & 1.146 & 292.00 & 1.204 & 342.00 & 1.250 & 392.00 & 1.225 \\
\hline 243.00 & 1.138 & 293.00 & 1.204 & 343.00 & 1.250 & 393.00 & 1.225 \\
\hline 244.00 & 1.142 & 294.00 & 1.200 & 344.00 & 1.238 & 394.00 & 1.225 \\
\hline 245.00 & 1.142 & 295.00 & 1.200 & 345.00 & 1.225 & 395.00 & 1.225 \\
\hline 246.00 & 1.138 & 296.00 & 1.200 & 346.00 & 1.229 & 396.00 & 1.225 \\
\hline 247.00 & 1.138 & 297.00 & 1.200 & 347.00 & 1.233 & 397.00 & 1.225 \\
\hline 248.00 & 1.138 & 298.00 & 1.200 & 348.00 & 1.238 & 398.00 & 1.225 \\
\hline 249.00 & 1.138 & 299.00 & 1.200 & 349.00 & 1.238 & 399.00 & 1.221 \\
\hline 250.00 & 1.150 & 300.00 & 1.208 & 350.00 & 1.238 & 400.00 & 1.229 \\
\hline 251.00 & 1.150 & 301.00 & 1.213 & 351.00 & 1.250 & 401.00 & 1.233 \\
\hline 252.00 & 1.146 & 302.00 & 1.213 & 352.00 & 1.246 & 402.00 & 1.229 \\
\hline 253.00 & 1.146 & 303.00 & 1.217 & 353.00 & 1.233 & 403.00 & 1.238 \\
\hline 254.00 & 1.138 & 304.00 & 1.225 & 354.00 & 1.238 & 404.00 & 1.238 \\
\hline 255.00 & 1.142 & 305.00 & 1.225 & 355.00 & 1.242 & 405.00 & 1.238 \\
\hline 256.00 & 1.146 & 306.00 & 1.225 & 356.00 & 1.229 & 406.00 & 1.238 \\
\hline 257.00 & 1.150 & 307.00 & 1.225 & 357.00 & 1.233 & 407.00 & 1.233 \\
\hline 258.00 & 1.154 & 308.00 & 1.225 & 358.00 & 1.233 & 408.00 & 1.225 \\
\hline 259.00 & 1.150 & 309.00 & 1.221 & 359.00 & 1.233 & 409.00 & 1.225 \\
\hline
\end{tabular}


Table C14. Thermo-Gravimetric Analysis Data for $K$ Basin Fuel Sample Run $49 \mathrm{a}$ at $90^{\circ} \mathrm{C}$. (Page 3 of 6 )

\begin{tabular}{|c|c|c|c|c|c|c|c|}
\hline \multicolumn{8}{|c|}{ RUN NUMBER 49a AT $90 \mathrm{C}$ (cont.) } \\
\hline $\begin{array}{l}\text { TIME } \\
\text { (minutes) }\end{array}$ & \begin{tabular}{|c|}
$\begin{array}{c}\text { wt change } \\
\text { (mg) }\end{array}$ \\
\end{tabular} & $\begin{array}{l}\text { TIME } \\
\text { (minutes) }\end{array}$ & $\begin{array}{c}\text { wt change } \\
\text { (mg) }\end{array}$ & $\begin{array}{l}\text { TIME } \\
\text { (minutes) }\end{array}$ & $\begin{array}{c}\text { wh change } \\
(\mathrm{mg})\end{array}$ & \begin{tabular}{|l} 
TIME \\
(minutes)
\end{tabular} & $\begin{array}{c}\text { wt change } \\
\text { (mg) }\end{array}$ \\
\hline 410.00 & 1.225 & 4600.00 & 1.221 & 510.00 & 1.204 & 560.00 & 1.238 \\
\hline 411.00 & 1.229 & 461.00 & 1.217 & 511.00 & 1.204 & 561.00 & 1.238 \\
\hline 412.00 & 1.238 & 462.00 & 1.217 & 512.00 & 1.200 & 562.00 & 1.242 \\
\hline 413.00 & 1.238 & 463.00 & 1.213 & 513.00 & 1.200 & 563.00 & 1.246 \\
\hline 414.00 & 1.238 & 464.00 & 1.213 & 514.00 & 1.200 & 564.00 & 1.229 \\
\hline 415.00 & 1.238 & 465.00 & 1.213 & 515.00 & 1.213 & 565.00 & 1.238 \\
\hline 416.00 & 1.225 & 466.00 & 1.208 & 516.00 & $1.217^{\circ}$ & 566.00 & 1.233 \\
\hline 417.00 & 1.225 & 467.00 & 1.208 & 517.00 & 1.213 & 567.00 & 1.246 \\
\hline 418.00 & 1.229 & 468.00 & 1.213 & 518.00 & 1.213 & 568.00 & 1.254 \\
\hline 419.00 & 1.233 & 469.00 & 1.204 & 519.00 & 1.213 & 569.00 & 1.246 \\
\hline 420.00 & 1.229 & 470.00 & 1.208 & 520.00 & 1.204 & 570.00 & 1.238 \\
\hline 421.00 & 1.226 & 471.00 & 1.213 & 521.00 & 1.213 & 571.00 & 1.238 \\
\hline 422.00 & 1.225 & 472.00 & 1.204 & 522.00 & 1.217 & 572.00 & 1.238 \\
\hline 423.00 & 1.225 & 473.00 & 1.208 & 523.00 & 1.225 & 573.00 & 1.233 \\
\hline 424.00 & 1.238 & 474.00 & 1.225 & 524.00 & 1.246 & 574.00 & 1.242 \\
\hline 425.00 & 7.242 & 475.00 & 1.213 & 525.00 & 1.242 & 575.00 & 1.238 \\
\hline 426.00 & 1.229 & 476.00 & 1.213 & 526.00 & 1.254 & 576.00 & 1.238 \\
\hline 427.00 & 1.238 & 477.00 & 1.200 & 527.00 & 1.263 & 577.00 & 1.238 \\
\hline 428.00 & 1.233 & 478.00 & 1.200 & 528.00 & 1.263 & 578.00 & 1.238 \\
\hline 429.00 & 1.238 & 479.00 & 1.200 & 529.00 & 1.254 & 579.00 & 1.229 \\
\hline 430.00 & 1.225 & 480.00 & 1.196 & 530.00 & 1.254 & 580.00 & 1.225 \\
\hline 431.00 & 1.225 & 481.00 & 1.208 & 531.00 & 1.250 & 581.00 & 1.229 \\
\hline 432.00 & 1.225 & 482.00 & 1.213 & 532.00 & 1.254 & 582.00 & 1.229 \\
\hline 433.00 & 1.225 & 483.00 & 1.213 & 533.00 & 1.238 & 583.00 & 1.225 \\
\hline 434.00 & 1.225 & 484.00 & 1.200 & 534.00 & 1.233 & 584.00 & 1.229 \\
\hline 435.00 & 1.225 & 485.00 & 1.200 & 535.00 & 1.225 & 585.00 & 1.225 \\
\hline 436.00 & 1.229 & 486.00 & 1.200 & 536.00 & 1.225 & 586.00 & 1.229 \\
\hline 437.00 & 1.229 & 487.00 & 1.208 & 537.00 & 1.225 & 587.00 & 1.233 \\
\hline 438.00 & 1.233 & 488.00 & 1.208 & 538.00 & 1.225 & 588.00 & 1.233 \\
\hline 439.00 & 1.233 & 489.00 & 1.213 & 539.00 & 1.238 & 589.00 & 1.238 \\
\hline 440.00 & 1.225 & 490.00 & 1.217 & 540.00 & 1.229 & 590.00 & 1.238 \\
\hline 441.00 & 1.229 & 491.00 & 1.213 & 541.00 & 1.225 & 591.00 & 1.238 \\
\hline 442.00 & 1.221 & 492.00 & 1.217 & 542.00 & 1.225 & 592.00 & 1.233 \\
\hline 443.00 & 1.217 & 493.00 & 1.221 & 543.00 & 1.225 & 593.00 & 1.238 \\
\hline 444.00 & 1.225 & 494.00 & 1.217 & 544.00 & 1.225 & 594.00 & 1.233 \\
\hline 445.00 & 1.213 & 495.00 & 1.217 & 545.00 & 1.263 & 595.00 & 1.238 \\
\hline 446.00 & 1.213 & 496.00 & 1.225 & 546.00 & 1.242 & 596.00 & 1.229 \\
\hline 447.00 & 1.213 & 497.00 & 1.213 & 547.00 & 1.238 & 597.00 & 1.225 \\
\hline 448.00 & 1.217 & 498.00 & 1.200 & 548.00 & 1.229 & 598.00 & 1.229 \\
\hline 449.00 & 1.213 & 499.00 & 1.200 & 549.00 & 1.217 & 599.00 & 1.238 \\
\hline 450.00 & 1.213 & 500.00 & 1.208 & 550.00 & 1.225 & 600.00 & 1.238 \\
\hline 451.00 & 1.213 & 501.00 & 1.213 & 551.00 & 1.229 & 601.00 & 1.229 \\
\hline 452.00 & 1.213 & 502.00 & 1.213 & 552.00 & 1.233 & 602.00 & 1.238 \\
\hline 453.00 & 1.204 & 503.00 & 1.213 & 553.00 & 1.238 & 603.00 & 1.233 \\
\hline 454.00 & 1.204 & 504.00 & 1.213 & 554.00 & 1.238 & 604.00 & 1.233 \\
\hline 455.00 & 1.213 & 505.00 & 1.213 & 555.00 & 1.238 & 605.00 & 1.233 \\
\hline 456.00 & 1.217 & 506.00 & 1.217 & 556.00 & 1.238 & 606.00 & 1.238 \\
\hline 457.00 & 1.213 & 507.00 & 1.213 & 557.00 & 1.233 & 607.00 & 1.238 \\
\hline 458.00 & 1.217 & 508.00 & 1.213 & 558.00 & 1.246 & 608.00 & 1.238 \\
\hline 459.00 & 1.217 & 509.00 & 1.208 & 559.00 & 1.250 & 609.00 & 1.242 \\
\hline
\end{tabular}


Table C14. Thermo-Gravimetric Analysis Data for $K$ Basin Fuel Sample Run $49 \mathrm{a}$ at $90^{\circ} \mathrm{C}$. (Page 4 of 6 )

\begin{tabular}{|c|c|c|c|c|c|c|c|}
\hline \multicolumn{8}{|c|}{ RUN NUMBER 49a AT $90 \mathrm{C}$ (cont.) } \\
\hline $\begin{array}{l}\text { TIME } \\
\text { (minutes) }\end{array}$ & \begin{tabular}{|c|} 
wt change \\
(mg)
\end{tabular} & $\begin{array}{l}\text { TIME } \\
\text { (minutes) }\end{array}$ & $\begin{array}{c}\text { wht change } \\
\text { (mg) }\end{array}$ & $\begin{array}{l}\text { TIME } \\
\text { (minutes) }\end{array}$ & \begin{tabular}{|c|}
$\begin{array}{c}\text { wh change } \\
\text { (mg) }\end{array}$ \\
\end{tabular} & $\begin{array}{l}\text { TIME } \\
\text { (minutes) }\end{array}$ & $\begin{array}{c}\text { wht change } \\
\text { (mg) }\end{array}$ \\
\hline 610.00 & 1.246 & 660.00 & 1.254 & 710.00 & 1.275 & 760.00 & 1.317 \\
\hline 611.00 & 1.246 & 661.00 & 1.250 & 711.00 & 1.288 & 761.00 & 1.308 \\
\hline 612.00 & 1.250 & 662.00 & 1.246 & 712.00 & 1.288 & 762.00 & 1.304 \\
\hline 613.00 & 1.238 & 663.00 & 1.250 & 713.00 & 1.288 & 763.00 & 1.313 \\
\hline 614.00 & 1.238 & 664.00 & 1.250 & 714.00 & 1.283 & 764.00 & 1.321 \\
\hline 615.00 & 1.238 & 665.00 & 1.246 & 715.00 & 1.275 & 765.00 & 1.317 \\
\hline 616.00 & 1.238 & 666.00 & 1.250 & 716.00 & 1.288 & 766.00 & 1.317 \\
\hline 617.00 & 1.225 & 667.00 & 1.242 & 717.00 & 1.275 & 767.00 & 1.317 \\
\hline 618.00 & 1.225 & 668.00 & 1.242 & 718.00 & 1.288 & 768.00 & 1.313 \\
\hline 619.00 & 1.225 & 669.00 & 1.250 & 719.00 & 1.288 & 769.00 & 1.317 \\
\hline 620.00 & 1.225 & 670.00 & 1.258 & 720.00 & 1.288 & 770.00 & 1.313 \\
\hline 621.00 & 1.225 & 671.00 & 1.250 & 721.00 & 1.288 & 771.00 & 1.313 \\
\hline 622.00 & 1.225 & 672.00 & 1.250 & 722.00 & 1.288 & 772.00 & 1.313 \\
\hline 623.00 & 1.238 & 673.00 & 1.258 & 723.00 & 1.288 & 773.00 & 1.313 \\
\hline 624.00 & 1.238 & 674.00 & 1.258 & 724.00 & 1.288 & 774.00 & 1.321 \\
\hline 625.00 & 1.225 & 675.00 & 1.267 & 725.00 & 1.283 & 775.00 & 1.321 \\
\hline 626.00 & 1.229 & 676.00 & 1.267 & 726.00 & 1.288 & 776.00 & 1.313 \\
\hline 627.00 & 1.238 & 677.00 & 1.275 & 727.00 & 1.288 & 777.00 & 1.313 \\
\hline 628.00 & 1.238 & 678.00 & 1.263 & 728.00 & 1.288 & 778.00 & $1.3 \uparrow 3$ \\
\hline 629.00 & 1.238 & 679.00 & 1.271 & 729.00 & 1.288 & 779.00 & 1.313 \\
\hline 630.00 & 1.225 & 680.00 & 1.258 & 730.00 & 1.300 & 780.00 & 1.308 \\
\hline 631.00 & 1.225 & 681.00 & 1.254 & 731.00 & 1.300 & 781.00 & 1.304 \\
\hline 632.00 & 1.225 & 682.00 & 1.263 & 732.00 & 1.300 & 782.00 & 1.308 \\
\hline 633.00 & 1.225 & 683.00 & 1.263 & 733.00 & 1.296 & 783.00 & 1.308 \\
\hline 634.00 & 1.229 & 684.00 & 1.263 & 734.00 & 1.288 & 784.00 & 1.313 \\
\hline 635.00 & 1.238 & 685.00 & 1.271 & 735.00 & 1.288 & 785.00 & 1.317 \\
\hline 636.00 & 1.246 & 686.00 & 1.271 & 736.00 & 1.296 & 786.00 & 1.308 \\
\hline 637.00 & 1.246 & 687.00 & 1.275 & 737.00 & 1.300 & 787.00 & 1.321 \\
\hline 638.00 & 1.246 & 688.00 & 1.271 & 738.00 & 1.300 & 788.00 & 1.317 \\
\hline 639.00 & 1.242 & 689.00 & 1.263 & 739.00 & 1.300 & 789.00 & 1.317 \\
\hline 640.00 & 1.238 & 690.00 & 1.263 & 740.00 & 1.313 & 790.00 & 1.321 \\
\hline 641.00 & 1.246 & 691.00 & 1.263 & 741.00 & 1.304 & 791.00 & 1.308 \\
\hline 642.00 & 7.254 & 692.00 & 1.258 & 742.00 & 1.313 & 792.00 & 1.304 \\
\hline 643.00 & 1.242 & 693.00 & 1.250 & 743.00 & 1.313 & 793.00 & 1.313 \\
\hline 644.00 & 1.238 & 694.00 & 1.250 & 744.00 & 1.317 & 794.00 & 1.321 \\
\hline 645.00 & 1.238 & 695.00 & 1.254 & 745.00 & 1.300 & 795.00 & 1.325 \\
\hline 646.00 & 1.238 & 696.00 & 1.258 & 746.00 & 1.304 & 796.00 & 1.329 \\
\hline 647.00 & 1.246 & 697.00 & 1.271 & 747.00 & 1.304 & 797.00 & 1.329 \\
\hline 648.00 & 1.246 & 698.00 & 1.275 & 748.00 & 1,313 & 798.00 & 1.325 \\
\hline 649.00 & 1.246 & 699.00 & 1.275 & 749.00 & 1.317 & 799.00 & 1.325 \\
\hline 650.00 & 1.250 & 700.00 & 1.271 & 750.00 & 1.317 & 800.00 & 1.321 \\
\hline 651.00 & 1.250 & 701.00 & 1.271 & 751.00 & 1.300 & 801.00 & 1.313 \\
\hline 652.00 & 1.250 & 702.00 & 1.275 & 752.00 & 1.300 & 802.00 & 1.313 \\
\hline 653.00 & 1.250 & 703.00 & 1.279 & 753.00 & 1.317 & 803.00 & 1.313 \\
\hline 654.00 & 1.246 & 704.00 & 1.267 & 754.00 & 1.325 & 804.00 & 1.313 \\
\hline 655.00 & 1.238 & 705.00 & 1.267 & 755.00 & 1.325 & 805.00 & 1.313 \\
\hline 656.00 & 1.238 & 706.00 & 1.275 & 756.00 & 1.313 & 806.00 & 1.313 \\
\hline 657.00 & 1.238 & 707.00 & 1.288 & 757.00 & 1.313 & 807.00 & 1.313 \\
\hline 658.00 & 1.242 & 708.00 & 1.283 & 758.00 & 1.313 & 808.00 & 1.325 \\
\hline 659.00 & 1.250 & 709.00 & 1.279 & 759.00 & 1.313 & 809.00 & 1.321 \\
\hline
\end{tabular}


Table C14. Thermo-Gravimetric Analysis Data for $\mathrm{K}$ Basin Fuel Sample Run $49 \mathrm{a}$ at $90{ }^{\circ} \mathrm{C}$. (Page 5 of 6 )

\begin{tabular}{|c|c|c|c|c|c|c|c|}
\hline \multicolumn{8}{|c|}{ RUN NUMBER 49a AT $90 \mathrm{C}$ (cont.) } \\
\hline $\begin{array}{l}\text { TIME } \\
\text { (minutes) }\end{array}$ & $\begin{array}{c}\text { wt change } \\
\text { (mg) }\end{array}$ & $\begin{array}{l}\text { TIME } \\
\text { (minutes) }\end{array}$ & $\begin{array}{c}\text { wt change } \\
\text { (mg) }\end{array}$ & $\begin{array}{l}\text { TIME } \\
\text { (minutes) }\end{array}$ & \begin{tabular}{|c|}
$\begin{array}{c}\text { wt change } \\
(\mathrm{mg})\end{array}$ \\
\end{tabular} & $\begin{array}{l}\text { TIME } \\
\text { (minutes) }\end{array}$ & \begin{tabular}{|c|} 
wt change \\
(mg)
\end{tabular} \\
\hline 810.00 & 1.325 & 860.00 & 1.363 & 910.00 & 1.363 & 960.00 & 1.388 \\
\hline 811.00 & 1.325 & 861.00 & 1.363 & 911.00 & 1.358 & 961.00 & 1.383 \\
\hline 812.00 & 1.313 & 862.00 & 1.363 & 912.00 & 1.358 & 962.00 & 1.383 \\
\hline 813.00 & 1.308 & 863.00 & 1.367 & 913.00 & 1.350 & 963.00 & 1.375 \\
\hline 814.00 & 1.300 & 864.00 & $1.37 \uparrow$ & 914.00 & 1.354 & 964.00 & 1.379 \\
\hline 815.00 & 1.304 & 865.00 & 1.379 & 915.00 & 1.363 & 965.00 & 1.383 \\
\hline 816.00 & 1.304 & 866.00 & 1.375 & 916.00 & 1.363 & 966.00 & 1.388 \\
\hline 817.00 & 1.304 & 867.00 & 1.375 & 917.00 & 1.354 & 967.00 & 1.388 \\
\hline 818.00 & 1.313 & 868.00 & 1.371 & 918.00 & 1.338 & 968.00 & 1.388 \\
\hline 819.00 & 1.317 & 869.00 & 1.375 & 919.00 & 1.350 & 969.00 & 1.388 \\
\hline 820.00 & 1.325 & 870.00 & 1.363 & 920.00 & 1.350 & 970.00 & 1.392 \\
\hline 821.00 & 1.321 & 871.00 & 1.375 & 921.00 & 1.354 & 971.00 & 1.383 \\
\hline 822.00 & 1.329 & 872.00 & 1.367 & 922.00 & 1.363 & 972.00 & 1.375 \\
\hline 823.00 & 1.325 & 873.00 & 1.375 & 923.00 & 1.358 & 973.00 & 1.379 \\
\hline 824.00 & 1.333 & 874.00 & 1.371 & 924.00 & 1.363 & 974.00 & 1.375 \\
\hline 825.00 & 1.342 & 875.00 & 1.367 & 925.00 & 1.363 & 975.00 & 1.379 \\
\hline 826.00 & 1.338 & 876.00 & 1.371 & 926.00 & 1.363 & 976.00 & 1.383 \\
\hline 827.00 & 1.342 & 877.00 & 1.375 & 927.00 & 1.375 & 977.00 & 1.383 \\
\hline 828.00 & 1.338 & 878.00 & 1.379 & 928.00 & 1.375 & 978.00 & 1.367 \\
\hline 829.00 & 1.342 & 879.00 & 1.379 & 929.00 & 1.375 & 979.00 & 1.375 \\
\hline 830.00 & 1.346 & 880.00 & 1.367 & 930.00 & 1.367 & 980.00 & 1.371 \\
\hline 831.00 & 1.325 & 881.00 & 1.371 & 931.00 & 1.367 & 981.00 & .1 .379 \\
\hline 832.00 & 1.313 & 882.00 & 1.363 & 932.00 & 1.371 & 982.00 & 1.379 \\
\hline 833.00 & 1.321 & 883.00 & 1.354 & 933.00 & 1.363 & 983.00 & 1.379 \\
\hline 834.00 & 1.317 & 884.00 & 1.346 & 934.00 & 1.363 & 984.00 & .1 .383 \\
\hline 835.00 & 1.325 & 885.00 & 1.338 & 935.00 & 1.363 & 985.00 & 1.388 \\
\hline 836.00 & 1.329 & 886.00 & 1.354 & 936.00 & 1.375 & 986.00 & 1.388 \\
\hline 837.00 & 1.333 & 887.00 & 1.350 & 937.00 & 1.371 & 987.00 & 1.383 \\
\hline 838.00 & 1.333 & 888.00 & 1.350 & 938.00 & 1.363 & 988.00 & 1.371 \\
\hline 839.00 & 1.329 & 889.00 & 1.354 & 939.00 & 1.363 & 989.00 & 1.379 \\
\hline 840.00 & 1.333 & 890.00 & 1.358 & 940.00 & 1.367 & 990.00 & 1.379 \\
\hline 841.00 & 1.329 & 891.00 & 1.363 & 941.00 & 1.371 & 991.00 & 1.383 \\
\hline 842.00 & 1.338 & 892.00 & 1.363 & 942.00 & 1.379 & 992.00 & 1.383 \\
\hline 843.00 & 1.338 & 893.00 & 1.367 & 943.00 & 1.375 & 993.00 & 1.379 \\
\hline 844.00 & 1.338 & 894.00 & 1.363 & 944.00 & 1.375 & 994.00 & 1.375 \\
\hline 845.00 & 1.350 & 895.00 & 1.367 & 945.00 & 1.371 & 995.00 & 1.375 \\
\hline 846.00 & 1.350 & 896.00 & 1.354 & 946.00 & 1.379 & 996.00 & 1.379 \\
\hline 847.00 & 1.354 & 897.00 & 1.350 & 947.00 & 1.379 & 997.00 & 1.388 \\
\hline 848.00 & 1.350 & 898.00 & 1.363 & 948.00 & 1.375 & 998.00 & 1.388 \\
\hline 849.00 & 1.350 & 899.00 & 1.350 & 949.00 & 1.371 & 999.00 & 1.383 \\
\hline 850.00 & 1.350 & 800.00 & 1.358 & 950.00 & 1.371 & 1000.00 & 1.383 \\
\hline 851.00 & 1.338 & 901.00 & 1.350 & 951.00 & 1.379 & 1001.00 & 1.388 \\
\hline 852.00 & 1.338 & 902.00 & 1.333 & 952.00 & 1.392 & 1002.00 & 1.367 \\
\hline 853.00 & 1.342 & 903.00 & 1.350 & 953.00 & 1.388 & 1003.00 & 1.371 \\
\hline 854.00 & 1.350 & 904.00 & 1.367 & 954.00 & 1.388 & 1004.00 & 1.375 \\
\hline 855.00 & 1.350 & 905.00 & 1.363 & 955.00 & 1.379 & 1005.00 & 1.375 \\
\hline 856.00 & 1.350 & 906.00 & 1.367 & 956.00 & 1.379 & 1006.00 & 1.379 \\
\hline 857.00 & 1.358 & 907.00 & 1.358 & 957.00 & 1.379 & 1007.00 & 1.375 \\
\hline 858.00 & 1.363 & 908.00 & 1.363 & 958.00 & 1.388 & 1008.00 & 1.363 \\
\hline 859.00 & 1.363 & 909.00 & 1.363 & 959.00 & 1.383 & 1009.00 & 1.371 \\
\hline
\end{tabular}


Table C14. Thermo-Gravimetric Analysis Data for K Basin

Fuel Sample Run $49 \mathrm{a}$ at $90{ }^{\circ} \mathrm{C}$. (Page 6 of 6 )

\begin{tabular}{|c|c|c|c|c|c|c|c|}
\hline \multicolumn{8}{|c|}{ RUN NUMBER 49a AT $90 \mathrm{C}$ (cont.) } \\
\hline $\begin{array}{l}\text { TIME } \\
\text { (minutes) }\end{array}$ & \begin{tabular}{|c}
$\begin{array}{c}w \text { change } \\
\text { (mg) }\end{array}$ \\
\end{tabular} & $\begin{array}{l}\text { TIME } \\
\text { (minutes) }\end{array}$ & $\begin{array}{c}\begin{array}{c}\text { wh change } \\
\text { (mg) }\end{array} \\
\end{array}$ & $\begin{array}{l}\text { TIME } \\
\text { (minutes) }\end{array}$ & $\begin{array}{c}\text { wt change } \\
\text { (mg) }\end{array}$ & $\begin{array}{l}\text { TIME } \\
\text { (minutes) }\end{array}$ & $\begin{array}{c}\text { wt change } \\
\text { (mg) }\end{array}$ \\
\hline 1010.00 & 1.375 & 1060.00 & 1.400 & 1110.00 & 1.400 & 1160.00 & 1.413 \\
\hline 1011.00 & 1.375 & 1061.00 & 1.388 & 1111.00 & 1.388 & 1161.00 & 1.413 \\
\hline 1012.00 & 1.379 & 1062.00 & 1.388 & 1112.00 & 1.388 & 1162.00 & 1.417 \\
\hline 1013.00 & 1.371 & 1063.00 & 1.388 & .1113 .00 & 1.388 & 1163.00 & 1.425 \\
\hline 1014.00 & 1.371 & 1064.00 & 1.388 & 1114.00 & 1.388 & 1164.00 & 1.417 \\
\hline 1015.00 & 1.363 & 1065.00 & 1.383 & 1115.00 & 1.392 & 1165.00 & 1.421 \\
\hline 1016.00 & 1.367 & 1066.00 & 1.379 & 1116.00 & 1.388 & 1166.00 & 1.404 \\
\hline 1017.00 & 1.371 & 1067.00 & 1.392 & 1117.00 & 1.396 & 1167.00 & 1.400 \\
\hline 1018.00 & 1.375 & 1068.00 & 1.392 & 1118.00 & 1.400 & 1168.00 & 1.400 \\
\hline 1019.00 & 1.371 & 1069.00 & 1.392 & 1119.00 & 1.396 & 1169.00 & 1.400 \\
\hline 1020.00 & 1.375 & 1070.00 & 1.396 & 1120.00 & 1.396 & 1170.00 & 1.413 \\
\hline 1021.00 & 1.367 & 1071.00 & 1.388 & 1121.00 & 1.400 & 1171.00 & 1.413 \\
\hline 1022.00 & 1.363 & 1072.00 & 1.396 & 1122.00 & 1.400 & 1172.00 & 1.404 \\
\hline 1023.00 & 1.367 & 1073.00 & 1.383 & 1123.00 & 1.400 & 1173.00 & 1.413 \\
\hline 1024.00 & 1.363 & 1074.00 & 1.388 & 1124.00 & 1.400 & 1174.00 & 1.400 \\
\hline 1025.00 & 1.358 & 1075.00 & 1.379 & 1125.00 & 1.404 & 1175.00 & 1.400 \\
\hline 1026.00 & 1.367 & 1076.00 & 1.379 & 1126.00 & 1.400 & 1176.00 & 1.400 \\
\hline 1027.00 & 1.375 & 1077.00 & 1.383 & 1127.00 & 1.396 & 1177.00 & 1.408 \\
\hline 1028.00 & 1.375 & 1078.00 & 1.379 & 1128.00 & 1.400 & 1178.00 & 1.404 \\
\hline 1029.00 & 1.379 & 1079.00 & 1.388 & 1129.00 & 1.400 & 1179.00 & 1.417 \\
\hline 1030.00 & 1.388 & 1080.00 & 1.388 & 1130.00 & 1.396 & 1180.00 & 1.413 \\
\hline 1031.00 & 1.375 & 1081.00 & 1.388 & 1131.00 & 1.396 & 1181.00 & 1.413 \\
\hline 1032.00 & 1.375 & 1082.00 & 1.388 & 1132.00 & 1.396 & 1182.00 & 1.408 \\
\hline 1033.00 & 1.379 & 1083.00 & 1.388 & 1133.00 & 1.400 & 1183.00 & 1.413 \\
\hline 1034.00 & 1.375 & 1084.00 & 1.388 & 1134.00 & 1.400 & 1184.00 & 1.408 \\
\hline 1035.00 & 1.375 & 1085.00 & 1.388 & 1135.00 & 1.400 & 1185.00 & 1.417 \\
\hline 1036.00 & 1.375 & 1086.00 & 1.388 & 1136.00 & 1.400 & 1186.00 & 1.425 \\
\hline 1037.00 & 1.375 & 1087.00 & 1.396 & 1137.00 & 1.404 & 1187.00 & 1.425 \\
\hline 1038.00 & 1.379 & 1088.00 & 1.388 & 1138.00 & 1.404 & 1188.00 & 1.413 \\
\hline 1039.00 & 1.375 & 1089.00 & 1.383 & 1139.00 & 1.400 & 1189.00 & 1.421 \\
\hline 1040.00 & 1.375 & 1090.00 & 1.383 & 1140.00 & 1.396 & 1190.00 & 1.417 \\
\hline 1041.00 & 1.379 & 1091.00 & 1.383 & 1141.00 & 1.396 & 1191.00 & 1.417 \\
\hline 1042.00 & 1.379 & 1092.00 & 1.375 & 1142.00 & 1.400 & 1192.00 & 1.429 \\
\hline 1043.00 & 1.375 & 1093.00 & 1.383 & 1143.00 & 1.404 & 1193.00 & 1.438 \\
\hline 1044.00 & 1.375 & 1094.00 & 1.388 & 1144.00 & 1.400 & 1194.00 & 1.429 \\
\hline 1045.00 & 1.375 & 1095.00 & 1.396 & 1145.00 & 1.400 & 1195.00 & 1.425 \\
\hline 1046.00 & 1.375 & 1096.00 & 1.400 & 1146.00 & 1.404 & 1196.00 & 1.425 \\
\hline 1047.00 & 379 & 1097.00 & 1.400 & 1147.00 & 1.400 & 1197.00 & 1.413 \\
\hline 1048.00 & 1.375 & 1098.00 & 1.400 & 1148.00 & 1.413 & 1198.00 & 1.408 \\
\hline 1049.00 & 1.367 & 1099.00 & 1.400 & 1149.00 & 1.417 & 1199.00 & 1.413 \\
\hline 1050.00 & 1.367 & 1100.00 & 1.400 & 1150.00 & 1.425 & 1200.00 & 1.408 \\
\hline 1051.00 & 1.375 & 1101.00 & 1.388 & 1151.00 & 1.413 & 1201.00 & 1.413 \\
\hline 1052.00 & 1.379 & 1102.00 & 1.392 & 1152.00 & 1.413 & 1202.00 & 1.413 \\
\hline 1053.00 & 1.388 & 1103.00 & 1.388 & 1153.00 & 1.408 & 1203.00 & 1.421 \\
\hline 1054.00 & 1.388 & 1104.00 & 1.396 & 1154.00 & 1.413 & 1204.00 & 1.421 \\
\hline 1055.00 & 1.388 & 1105.00 & 1.396 & 1155.00 & 1.400 & 1205.00 & 1.433 \\
\hline 1056.00 & 1.388 & 1106.00 & 1.392 & 1156.00 & 1.408 & 1206.00 & 1.438 \\
\hline 1057.00 & 1.388 & 1107.00 & 7.400 & 1157.00 & 1.413 & 1207.00 & 1.429 \\
\hline 1058.00 & 1.392 & 1108.00 & 1.400 & 1158.00 & 1.417 & 1208.00 & 1.421 \\
\hline 1059.00 & 1.408 & 1109.00 & 1.400 & 1159.00 & 1.425 & 1209.00 & 1.429 \\
\hline
\end{tabular}


HNF-SD-SNF-CN-035, Rev. 0

Table C15. Thermo-Gravimetric Analysis Data for K Basin Fuel Sample Run $49 \mathrm{~b}$ at $124{ }^{\circ} \mathrm{C}$.

\begin{tabular}{|c|c|c|c|c|c|}
\hline \multicolumn{6}{|c|}{ RUN NUMBER 49b AT $124 \mathrm{C}$} \\
\hline $\begin{array}{c}\text { TIME } \\
\text { (minutes) }\end{array}$ & $\begin{array}{c}\text { wt change } \\
\text { (mg) }\end{array}$ & $\begin{array}{l}\text { TIME } \\
\text { (minutes) }\end{array}$ & $\begin{array}{c}\text { wt change } \\
(\mathrm{mg})\end{array}$ & $\begin{array}{l}\text { TIME } \\
\text { (minutes) }\end{array}$ & $\begin{array}{c}\text { wt change } \\
\text { (mg) }\end{array}$ \\
\hline 20.00 & 0.9875 & 353.33 & $\overline{6.4833}$ & 686.67 & 43.2750 \\
\hline 26.67 & 1.1458 & 360.00 & 6.5333 & 693.33 & 43.3250 \\
\hline 33.33 & 1.4542 & 366.67 & 6.5750 & 700.00 & 43.4210 \\
\hline 40.00 & 1.7417 & 373.33 & 6.6167 & 706.67 & 43.5000 \\
\hline 46.67 & 2.0083 & 380.00 & 6.6583 & 713.33 & 43.5630 \\
\hline 53.33 & 2.2625 & 386.67 & 6.7042 & 720.00 & 43.5920 \\
\hline 60.00 & 2.5042 & 393.33 & 6.7458 & 726.67 & 43.6250 \\
\hline 66.67 & 2.7333 & 400.00 & 6.7833 & 733.33 & 43.6540 \\
\hline 73.33 & 2.9458 & 406.67 & 6.8125 & 740.00 & 43.6670 \\
\hline 80.00 & 3.1458 & 413.33 & 6.8333 & 746.67 & 43.6710 \\
\hline 86.67 & 3.3292 & 420.00 & 6.8542 & 753.33 & 43.6750 \\
\hline 93.33 & 3.4917 & 426.67 & 6.8750 & 760.00 & 43.6710 \\
\hline 100.00 & 3.6292 & 433.33 & 6.9000 & 766.67 & 43.6710 \\
\hline 106.67 & 3.7583 & 440.00 & 6.9250 & 773.33 & 43.6750 \\
\hline 113.33 & 3.8833 & 446.67 & 6.9500 & 780.00 & 43.6920 \\
\hline 120.00 & 4.0083 & 453.33 & 6.9708 & 786.67 & 43.7080 \\
\hline 126.67 & 4.1250 & 460.00 & 6.9917 & 793.33 & 43.7290 \\
\hline 133.33 & 4.2375 & 466.67 & 7.0125 & 800.00 & 43.7380 \\
\hline 140.00 & 4.3458 & 473.33 & 7.0417 & 806.67 & 43.7460 \\
\hline 146.67 & 4.4417 & 480.00 & 7.0750 & 813.33 & 43.7500 \\
\hline 153.33 & 4.5333 & 486.67 & 7.1125 & 820.00 & 43.7630 \\
\hline 160.00 & 4.6208 & 493.33 & 7.1583 & 826.67 & 43.7670 \\
\hline 166.67 & 4.7167 & 500.00 & 7.2042 & 833.33 & 43.7710 \\
\hline 173.33 & 4.8083 & 506.67 & 7.2542 & 840.00 & 43.7790 \\
\hline 180.00 & 4.8958 & 513.33 & 7.2958 & 846.67 & 43.7920 \\
\hline 186.67 & 4.9792 & 520.00 & 7.3333 & 853.33 & 43.8000 \\
\hline 193.33 & 5.0625 & 526.67 & 7.3708 & 860.00 & 43.8040 \\
\hline 200.00 & 5.1375 & 533.33 & 7.4042 & 866.67 & 43.8040 \\
\hline 206.67 & 5.2083 & 540.00 & 7.4417 & 873.33 & 43.8080 \\
\hline 213.33 & 5.2750 & 546.67 & 7.4708 & 880.00 & 43.8040 \\
\hline 220.00 & 5.3458 & 553.33 & 7.5167 & 886.67 & 43.8130 \\
\hline 226.67 & 5.4125 & 560.00 & 7.5583 & 893.33 & 43.8130 \\
\hline 233.33 & 5.4792 & 566.67 & 7.6042 & 900.00 & 43.8130 \\
\hline 240.00 & 5.5500 & 573.33 & 7.6375 & 906.67 & 43.8000 \\
\hline 246.67 & 5.6208 & 580.00 & 7.6708 & & \\
\hline 253.33 & 5.6875 & 586.67 & 7.7000 & & \\
\hline 260.00 & 5.7458 & 593.33 & 7.7333 & & \\
\hline 266.67 & 5.8083 & 600.00 & 7.7625 & & \\
\hline 273.33 & 5.8708 & 606.67 & 7.7917 & & \\
\hline 280.00 & 5.9333 & 613.33 & 7.8125 & & \\
\hline 286.67 & 5.9875 & 620.00 & 7.8333 & & \\
\hline 293.33 & 6.0417 & 626.67 & 7.8542 & & \\
\hline 300.00 & 6.0958 & 633.33 & 7.8708 & & \\
\hline 306.67 & 6.1542 & 640.00 & 12.5830 & & \\
\hline 313.33 & 6.2083 & 646.67 & 21.2000 & & \\
\hline 320.00 & 6.2583 & 653.33 & 31.5710 & & \\
\hline 326.67 & 6.3083 & 660.00 & 38.2790 & & \\
\hline 333.33 & 6.3542 & 666.67 & 41.3880 & & \\
\hline 340.00 & 6.3958 & 673.33 & 42.8000 & & \\
\hline 346.67 & 6.4375 & 680.00 & 43.1830 & & \\
\hline
\end{tabular}


Table C16. Thermo-Gravimetric Analysis Data for $K$ Basin Fuel Sample Run 51 at $69{ }^{\circ} \mathrm{C}$. (Page 1 of 7 )

\begin{tabular}{|c|c|c|c|c|c|c|c|c|c|c|c|}
\hline \multicolumn{12}{|c|}{ RUN NUMBER 51 AT $69 \mathrm{C}$} \\
\hline $\begin{array}{c}\text { TME } \\
\text { (minutes) }\end{array}$ & $\begin{array}{c}\text { Wh change } \\
\text { (mg) }\end{array}$ & $\begin{array}{l}\text { TiME } \\
\text { (minutes) }\end{array}$ & $\begin{array}{c}\text { Wt change } \\
\text { (mg) }\end{array}$ & $\begin{array}{l}\text { TIME } \\
\text { (minutes) }\end{array}$ & $\begin{array}{c}\text { wt change } \\
\text { (mg) }\end{array}$ & $\begin{array}{l}\text { TाME } \\
\text { (minutes) }\end{array}$ & $\begin{array}{c}\text { wtchange } \\
\text { (mg) }\end{array}$ & $\begin{array}{l}\text { TlME } \\
\text { (minutes) }\end{array}$ & \begin{tabular}{|c|} 
wt change \\
(mg)
\end{tabular} & $\begin{array}{l}\text { TiME } \\
\text { (minutes) }\end{array}$ & $\begin{array}{c}\text { witchange } \\
\text { (min) }\end{array}$ \\
\hline 65.00 & 0.5875 & 183.33 & 2.4708 & 301.67 & 3.1250 & 420.00 & 3.3875 & 538.33 & 3.5375 & 656.67 & 3.7708 \\
\hline 66.67 & 0.6000 & 185.00 & 2.4958 & 303.33 & 3.1375 & 421.67 & 3.4083 & 540.00 & 3.5292 & 658.33 & 3.7687 \\
\hline 68.33 & 0.6083 & 186.67 & 2.5083 & 305.00 & 3.1375 & 423.33 & 3.4042 & 541.67 & 3.5458 & 660.00 & 3.7833 \\
\hline 70.00 & 0.6083 & 188.33 & 2.5208 & 306.67 & 3.1500 & 425.00 & 3.4208 & 543.33 & 3.5750 & 661.67 & 3.7792 \\
\hline 71.67 & 0.6042 & 190.00 & 2.5417 & 300.33 & 3.1500 & 426.67 & 3.4292 & 545.00 & 3.5792 & 663.33 & 3.7708 \\
\hline 73.33 & 0.6042 & 191.67 & 2.5708 & 310.00 & 3.1583 & 428.33 & 3.4375 & 546.67 & 3.5792 & 665.00 & 3.7375 \\
\hline 75.00 & 0.5958 & 193.33 & 2.5917 & 311.67 & 3.1625 & 430.00 & 3.3917 & 548.33 & 3.5667 & 666.67 & 3.7292 \\
\hline 76.67 & 0.6083 & 195.00 & 2.6083 & 313.33 & 3.1667 & 431.67 & 3.3833 & 550.00 & 3.5917 & 668.33 & 3.7202 \\
\hline 78.33 & 0.6208 & 196.67 & 26125 & 315.00 & 3.1708 & 433.33 & 3.3833 & 551.67 & 3.5833 & 670.00 & 3.7417 \\
\hline 80.00 & 0.6458 & 198.33 & 2.6458 & 316.67 & 3.1750 & 435.00 & 3.3917 & 553.33 & 3.5917 & 671.67 & 3.7500 \\
\hline 81.67 & 0.6542 & 200.00 & 2.6583 & 318.33 & 3.1833 & 436.67 & 3.3917 & 555.00 & 3.5708 & 673.33 & 3.7625 \\
\hline 83.33 & 0.6458 & 201.67 & 2.6708 & 320.00 & 3.1917 & 438.33 & 3.3958 & 556.67 & 3.5750 & 675.00 & 3.7875 \\
\hline 85.00 & 0.6583 & 203.33 & 26750 & 321.67 & 3.1958 & 440.00 & 3.4375 & 558.33 & 3.5875 & 676.67 & 3.8125 \\
\hline 86.67 & 0.6417 & 205.00 & 26708 & 323.33 & 3.1917 & 441.67 & 3.4333 & 560.00 & 3.5958 & 678.33 & 3.8375 \\
\hline 88.33 & 0.6792 & 206.67 & 2.6750 & 325.00 & 3.1958 & 443.33 & 3.4375 & 561.67 & 3.5917 & 680.00 & 3.8333 \\
\hline 90.00 & 0.6833 & 208.33 & 2.6958 & 326.67 & 3.2000 & 445.00 & 34083 & 563.33 & 3.5625 & 681.67 & 3.8250 \\
\hline 91.67 & 0.7497 & 210.00 & 27167 & 328.33 & 3.2083 & 446.67 & 3.4000 & 565.00 & 3.5875 & 683.33 & 3.8000 \\
\hline 93.33 & 0.7625 & 211.67 & 2.7333 & 330.00 & 3.2167 & 448.33 & 3.4125 & 566.67 & 3.5917 & 685.00 & 3.7875 \\
\hline 95.00 & 0.8375 & 213.33 & 2.7292 & 331.67 & 3.2125 & 450.00 & 3.4417 & 568.33 & 3.6292 & 686.67 & 3.7708 \\
\hline 96.67 & 0.8667 & 215.00 & 2.7333 & 333.33 & 3.2042 & 451.67 & 3.4525 & 570.00 & 3.6250 & 688.33 & 3.7667 \\
\hline 98.33 & 0.9208 & 216.67 & 27500 & 335.00 & 3.1958 & 453.33 & 3.4417 & 571.67 & 3.6500 & 690.00 & 3.7667 \\
\hline 100.00 & 0.9250 & 218.33 & 27792 & 336.67 & 3.2250 & 455.00 & 3.4125 & 573.33 & 3.6583 & 691.67 & 3.7792 \\
\hline 101.67 & 0.9917 & 220.00 & 2.7958 & 338.33 & 3.2292 & 456.67 & 3,4292 & 575,00 & 3.6667 & 693.33 & 3.8083 \\
\hline 103.33 & 1.0250 & 221.67 & 2,8042 & 340.00 & 3.2292 & 458.33 & 3.4500 & 576.67 & 3.6667 & 695.00 & 3.8033 \\
\hline 105.00 & 1.0667 & 223.33 & 2.8000 & 341.67 & 3.2375 & 460.00 & 3.4708 & 578.33 & 3.6542 & 696.67 & 3.8083 \\
\hline 106.67 & 1.0750 & 225.00 & 2.8167 & 343.33 & 3.2542 & 461.67 & 3.4500 & 580.00 & 3.6250 & 698.33 & 3.7958 \\
\hline 103.33 & 1.1125 & 226.67 & 2.8333 & 345.00 & 3.2667 & 463.33 & 3.4833 & 581.67 & 3.6042 & 700.00 & 3.7875 \\
\hline 110.00 & 1.1542 & 228.33 & 2.8458 & 346.67 & 3.2458 & 465.00 & 3.4917 & 583.33 & 3.5875 & 701.67 & 3.7792 \\
\hline 111.67 & 1.2000 & 230.00 & 2.8417 & 348.33 & 3.2583 & 466.67 & 3.5167 & 585.00 & 3.6125 & 703.33 & 3.7625 \\
\hline 113.33 & 1.2167 & 231.67 & 2.8458 & 350.00 & 3.2667 & 468.33 & 3.4917 & 586.67 & 3.6375 & 705.00 & 3.7750 \\
\hline 115.00 & 1.2333 & 233.33 & 2.8500 & 351.67 & 3.2708 & 470.00 & 3.5042 & 588.33 & 3.6417 & 706.67 & 3.7792 \\
\hline 116.67 & 1.2583 & 235.00 & 2.8625 & 353.33 & 3.2917 & 471.67 & 3.4875 & 590.00 & 3.6375 & 708.33 & 3.7917 \\
\hline 118.33 & 1.3125 & 236.67 & 2.8833 & 355.00 & 3.2833 & 473.33 & 3.4667 & 591.67 & 3.6250 & 710.00 & 3.8042 \\
\hline 120.00 & 1.3667 & 238.33 & 2.9083 & 356.67 & 3.3000 & 475.00 & 3.4708 & 593.33 & 3.6458 & 711.67 & 3.8125 \\
\hline 121.67 & 1.4208 & 240.00 & 2.9333 & 358.33 & 3.2875 & 476.67 & 3.4667 & 595.00 & 3.6708 & 713.33 & 3.8167 \\
\hline 123.33 & 1.4583 & 241.67 & 2.9250 & 360.00 & 3.2958 & 478.33 & 3.4958 & 596.67 & 3.6958 & 715.00 & 3.8042 \\
\hline 125.00 & 1.4917 & 243.33 & 2.9250 & 361.67 & 3.2958 & 480.00 & 3,4708 & 598.33 & 3.7125 & 716.67 & 3.7958 \\
\hline 126.67 & 1.5250 & 245.00 & 2.9250 & 363.33 & 3.3000 & 481.67 & 3.4458 & 600.00 & 3.6917 & 718.33 & 3.8125 \\
\hline 128.33 & 1.5917 & 246.67 & 2,9417 & 365.00 & 3.3208 & 483.33 & 3.4125 & 601.67 & 3.6708 & 720.00 & 3.8208 \\
\hline 130.00 & 1.6167 & 248.33 & 2.9500 & 366.67 & 3.3208 & 485.00 & 3.4125 & 603.33 & 3.6667 & 721.67 & 3.8250 \\
\hline 131.67 & 1.6208 & 250.00 & 2.9750 & 368.33 & 3.3417 & 486.67 & 3.4583 & 605.00 & 3.6792 & 723.33 & 3.8208 \\
\hline 133.33 & 1.6208 & 251.67 & 2.9708 & 370.00 & 3.3458 & 488.33 & 3.4625 & 606.67 & 3.7083 & 725.00 & 3.8208 \\
\hline 135.00 & 1.6583 & 253.33 & 2.9625 & 371.67 & 3.3458 & 490.00 & 3.4542 & 608.33 & 3.7042 & 726.67 & 3.8417 \\
\hline 136.67 & 1.7167 & 255.00 & 2.9458 & 373.33 & 3.3252 & 491.67 & 3.4333 & 610.00 & 3.6917 & 728.33 & 3.8375 \\
\hline 138.33 & 1.7625 & 256.67 & 2.9583 & 375.00 & 3.3250 & 493.33 & 3.4375 & 611.67 & 3.6917 & 730.00 & 3.8375 \\
\hline 140.00 & 1.8187 & 268.33 & 2.9667 & 376.67 & 3.3167 & 495.00 & 3.4657 & 613.33 & 3.6833 & 731.67 & 3.8333 \\
\hline 141.67 & 1.8667 & 260.00 & 2.9750 & 378.33 & 3.3333 & 496.67 & 3.4833 & 615.00 & 3.6958 & 733.33 & 3.8375 \\
\hline 143.33 & 1.9000 & 261.67 & 2.9833 & 380.00 & 3.3250 & 498.33 & 3.6083 & 816.67 & 3.6958 & 735.00 & 3.8542 \\
\hline 145,00 & 1,9417 & 263.33 & 3.0042 & 381.67 & 3.3250 & 500.00 & 3.5167 & 618.33 & 3.7083 & 736.67 & 3.8458 \\
\hline 146.67 & 1.9667 & $265.00^{\circ}$ & 3.0250 & 383.33 & 3.3042 & 501.67 & 3.6083 & 620.00 & 3.7042 & 738.33 & 3.8468 \\
\hline 148.33 & 1.9958 & 266.67 & 3.0375 & 385.00 & 3.3125 & 506.33 & 3.4792 & 621.67 & 3.7000 & 740.00 & 3.8292 \\
\hline 150,00 & 2.0333 & 268.33 & 3.0417 & 386.67 & 3.3208 & 505.00 & 3.4625 & 623.33 & 3.7000 & 741.67 & 3.8250 \\
\hline 151.67 & 2.0657 & 270.00 & 3.0333 & 388.33 & 3.3292 & 506.67 & 3.4667 & 625.00 & 3.7125 & 743.33 & 3.8250 \\
\hline 153.33 & 2,1083 & 271,67 & 3.0333 & 390.00 & 3.3125 & 506.33 & 3.4875 & 626.67 & 3.6917 & 745.00 & 3.8292 \\
\hline 155.00 & 2.1167 & 273.33 & 3.0292 & 391.67 & 3.3167 & 510.00 & 3.4833 & 628.33 & 3.6958 & 746.67 & 3.8333 \\
\hline 156.67 & 2.1458 & 275,00 & 3.0458 & 393.33 & & & 3.4 & 630.00 & 3.7208 & 748.33 & 3.8333 \\
\hline 158.33 & 2.1667 & 276.67 & 3.0500 & 39500 & 3.3000 & 513.33 & 3.4875 & 631.67 & 3.7417 & 750.00 & $3.845 \overline{8}$ \\
\hline 160.00 & 2.1875 & 278.33 & & 396.67 & 3.3042 & & 3.5042 & 633.33 & 3.7458 & 751.67 & 3.8542 \\
\hline 161.67 & 2.2000 & 280.00 & 3.0583 & 398.33 & 3.3500 & 516.67 & 3.5125 & 635.00 & 3.7125 & 753.33 & 3.8583 \\
\hline 163.33 & 2.2083 & 281.67 & 3.0625 & 400.00 & 3.3667 & & 3.5042 & 836.67 & 3.7042 & 755,00 & 3.8542 \\
\hline 165.00 & 2.2458 & 283,33 & 3.0583 & 401.67 & 3.3667 & 520.00 & 3.5208 & 638.33 & 3.7250 & 756.67 & $3.8750^{\circ}$ \\
\hline 166.67 & 2.2067 & 285.00 & 3.0708 & 403.33 & 17 & 521.67 & 3.5375 & 640.00 & 3.7333 & 758.33 & 3.8833 \\
\hline 168.33 & 2.2958 & 286.67 & 3.0750 & 405.00 & 3.3333 & 523.33 & 3.5458 & 641.67 & 3.7625 & 760.00 & 3.8875 \\
\hline 170.00 & 2.3042 & 288.33 & 3.0875 & 406.67 & 3.3625 & 525.00 & 3.5375 & 643.33 & 3.7333 & 761.67 & 3.8708 \\
\hline 171.67 & 2.3375 & 250.00 & 3.1083 & 408.33 & 3.3708 & 526.67 & 3.5292 & 645,00 & 3.7542 & 763.33 & 3.8583 \\
\hline 173.33 & 2.3625 & 291.67 & 3.1250 & 410.00 & 3.3958 & 528.33 & 3.5458 & 646.67 & 3.7583 & 765.00 & 3.8625 \\
\hline 175.00 & 2.3833 & 293.33 & 3.1125 & 411.67 & 3.3833 & 530.00 & 3.5375 & 648.33 & 3.7750 & 766.67 & 3.8625 \\
\hline 176.67 & 2.3792 & 295.00 & 3.1042 & 413.33 & 3.3833 & 531.67 & 3.5333 & 650.00 & 3.7792 & 768.33 & 3.8708 \\
\hline 178.33 & 2.3958 & 296.67 & 3.1000 & 415.00 & 3.3750 & 533.33 & 3.5292 & 651.67 & 3.7667 & 770.00 & 38875 \\
\hline 180.00 & 2.4167 & 298.33 & 3.1125 & 416.67 & 3.3667 & 535.00 & 3.5417 & 653.33 & 3.7708 & 771.67 & 3.9083 \\
\hline 181,67 & 2.4583 & 300.00 & 3,1208 & 418.33 & 3.3708 & 536.67 & 3.5500 & 655.00 & 3.7625 & 773.33 & 3.9167 \\
\hline
\end{tabular}


Table C16. Thermo-Gravimetric Analysis Data for $K$ Basin Fuel Sample Run 51 at $69{ }^{\circ} \mathrm{C}$. (Page 2 of 7 )

\begin{tabular}{|c|c|c|c|c|c|c|c|c|c|c|c|}
\hline \multicolumn{12}{|c|}{ RUN NUMBER 51 AT $69 \mathrm{C}$ (cont.) } \\
\hline $\begin{array}{l}\text { TIME } \\
\text { (minutes) }\end{array}$ & $\begin{array}{c}\text { Wh change } \\
\text { (mg) }\end{array}$ & $\begin{array}{l}\text { TiME } \\
\text { (minutos) }\end{array}$ & $\begin{array}{c}\text { wh change } \\
\text { (mg) }\end{array}$ & $\begin{array}{l}\text { TIME } \\
\text { (minutes) }\end{array}$ & $\begin{array}{c}\text { Wh change } \\
\text { (mg) }\end{array}$ & $\begin{array}{l}\text { TIME } \\
\text { (minutes) }\end{array}$ & $\begin{array}{c}\text { Wh change } \\
\text { (mg) }\end{array}$ & $\begin{array}{l}\text { TiME } \\
\text { (minutes) }\end{array}$ & \begin{tabular}{|c|}
$\begin{array}{c}\text { Wt change } \\
\text { (mg) }\end{array}$ \\
\end{tabular} & $\begin{array}{l}\begin{array}{l}\text { TIME } \\
\text { (minutes) }\end{array} \\
\end{array}$ & $\begin{array}{c}\text { Wt change } \\
\text { (mg) }\end{array}$ \\
\hline 775.00 & 3.8958 & 893.33 & 3.9625 & 1011.67 & 4.0958 & 1130.00 & 4.2083 & 1248.33 & 4.2792 & 1366.67 & 4.3042 \\
\hline 776.67 & 3.8875 & 895.00 & 3.9708 & 1013.33 & 4.1125 & 1131.67 & 4.1958 & 1250.00 & 4.2750 & 1368.33 & 4.3125 \\
\hline 778.33 & 3.2833 & 896.67 & 3.9750 & 1015.00 & 4.1292 & 1133.33 & 4.1917 & 1251.67 & 4.2792 & 1370.00 & 4.3042 \\
\hline 780.00 & 3.8833 & 898.33 & 3.9792 & 1016.67 & 4.1125 & 1135.00 & 4.1958 & 1253.33 & 4.2058 & 1371.67 & 4.3083 \\
\hline 781.67 & 3.8708 & 900.00 & 3.9667 & 1018.33 & 4.1042 & 1136.67 & 4.2083 & 1255.00 & 4.3083 & 1373.33 & 4.3292 \\
\hline 783.33 & 3.8542 & 901.67 & 3.9708 & 1020.00 & 4.1000 & 1138.33 & 4.2250 & 1256.67 & 4.3042 & $\mathbf{1 3 7 5 . 0 0}$ & 4.3417 \\
\hline 785.00 & 3.8500 & 903.33 & 3.9667 & 1021.67 & 4.1125 & 1140.00 & 4.2458 & 1258.33 & 4.2792 & 1376.67 & 4.3625 \\
\hline 786.67 & 3.8417 & 905.00 & 3.9875 & 1023.33 & 4.1083 & 1141.67 & 4.2542 & 1260,00 & 4.2625 & 1378.33 & 4.3708 \\
\hline 788.33 & 3.8417 & 906.67 & 3.9917 & 1025.00 & 4.0058 & 1143.33 & 4.2583 & 1261.67 & 4.2750 & 138000 & 4.3833 \\
\hline 790.00 & 3.8375 & 908.33 & 3.9958 & 1026.67 & 4.1000 & 1145.00 & 4.2625 & 1263.33 & 4.3042 & 1381.67 & 4.3625 \\
\hline 791.67 & 3.8375 & 910.00 & 3.9792 & 1028.33 & 4.1000 & 1146.67 & 4.2542 & 1265.00 & 4.3083 & 1383.33 & 4.3375 \\
\hline 793.33 & 3.8417 & 911.67 & 3.9542 & 1030.00 & 4.1042 & 1148.33 & 4.2500 & 1266.67 & 4.2917 & 1385.00 & 4.3417 \\
\hline 795.00 & 3.8458 & 913.33 & 3.9417 & 1031.67 & 4.1167 & 1150.00 & 4.2458 & 1268.33 & 4.2750 & 1386.67 & 4.3542 \\
\hline 796.67 & 3.8542 & 915.00 & 3.9417 & 1033.33 & 4.1083 & 1157.67 & 4.2458 & 1270.00 & 4.2667 & 1388.33 & 4.3458 \\
\hline 798.33 & 3.8542 & 916.67 & 3.9583 & 1035.00 & 4.1167 & 1153.33 & 4.2542 & 1271.67 & 4.2833 & 1390.00 & 4.3500 \\
\hline 800.00 & 3.8542 & 918.33 & 3.9625 & 1036.67 & 4.1125 & 1155.00 & 4.2625 & 1273.33 & 4.2792 & 1391.67 & 4.3417 \\
\hline 801.87 & 3.8500 & 920.00 & 3.9625 & 1038.33 & 4.1333 & 1156.67 & 4.2708 & 1275.00 & 4.2917 & 1393.33 & 4.3500 \\
\hline 803.33 & 3.8542 & 921.67 & 3.9583 & 1040.00 & 4.1417 & 1158.33 & 4.2708 & 1276.67 & 4.3083 & 1395.00 & 4.3292 \\
\hline 805.00 & 3.8583 & 923.33 & 3.9542 & 9041.67 & 4.1542 & 1160.00 & 4.2708 & 1278.33 & 4.3333 & 1396.67 & 4.3333 \\
\hline 806.67 & 3.8625 & 925.00 & 3.0583 & 1043.33 & 4.1542 & 1161.67 & 4.2667 & 1280.00 & 4.3417 & 1398,33 & 4.3333 \\
\hline 809.33 & 3.8625 & 926.67 & 3.9542 & 1045.00 & 4.1542 & 1163.33 & 4.2667 & 1281.67 & 4.3375 & 1400.00 & 4.3417 \\
\hline 810.00 & 3.8625 & 928.33 & 3.9625 & 1046.67 & 4.1417 & 1165.00 & 4.2542 & 1283.33 & 4.3167 & 1401.67 & 4.3458 \\
\hline 811.67 & 3.3625 & 930.00 & 3.9583 & 1048.33 & 4.1292 & 1166.67 & 4.2625 & 1285.00 & 4.3042 & 1403.33 & 4.3458 \\
\hline 813.33 & 3.8750 & 931.67 & 3.9750 & 1050.00 & 4.1333 & 1168.33 & 4.2542 & 1286.67 & 4.2792 & 1405.00 & 4.3458 \\
\hline 815.00 & 3.8750 & 933.33 & 3.9833 & 1051.67 & 4.1333 & 1170.00 & 4.2708 & 1288.33 & 4.2958 & 1406.67 & 4.3583 \\
\hline 816.67 & 3.8792 & 935.00 & 3.9958 & 1053.33 & 4.1375 & 1171.67 & 4.2583 & 1290.00 & 4.3083 & 1408.33 & 4.3750 \\
\hline 818.33 & 3.8708 & 936.67 & 3.8875 & 1055.00 & 4.1167 & 1173.33 & 4.2583 & 1291.67 & 4.3167 & 1410.00 & 4.3792 \\
\hline 820.00 & 3.8667 & 938.33 & 3.9792 & 1056.67 & 4.1167 & 1175.00 & 4.2458 & 1293.33 & 4.3167 & 1411.67 & 4.3750 \\
\hline 821.67 & 3.8583 & 940.00 & 3.9708 & 1058.33 & 4.1417 & 1176.67 & 4.2500 & 1295.00 & 4.3125 & 1413.33 & 4.3708 \\
\hline 823.33 & 3.8833 & 941.67 & 3.9667 & 1060.00 & 4.1625 & 1178.33 & 4.2458 & 1296.67 & 4.3083 & 1415.00 & 4.3708 \\
\hline 825.00 & 3.8958 & 943.33 & 3.9750 & 1061.67 & 4.1792 & 1180.00 & 4.2542 & 1298.33 & 4.2917 & 1416.67 & 4.3708 \\
\hline 826.67 & 3.9208 & 945.00 & 3.9667 & 1063.33 & 4.1708 & 1181.67 & 4.2542 & 1300.00 & 4.2958 & 1418.33 & 4.3958 \\
\hline 828.33 & 3.9083 & 946.67 & 3.0667 & 1065.00 & 4.1625 & 1183.33 & 4.2583 & 1301.67 & 4.2875 & 1420.00 & 4.4042 \\
\hline 830.00 & 3.9083 & 948.33 & 3.9708 & 1066,67 & 4.1542 & 1185.00 & 4.2625 & 1303.33 & 4.3042 & 1421.67 & 4.4042 \\
\hline 831.67 & 3.9042 & 950.00 & 3.9792 & 1068.33 & 4.1583 & 1186.67 & 4.2792 & 1305.00 & 4.3042 & 1423.33 & 4.3708 \\
\hline 833.33 & 3.8958 & 951.67 & 3.9833 & 1070.00 & 4.1792 & 1188.33 & 4.3000 & 1306.67 & 4.3250 & 1425,00 & 4.3625 \\
\hline 835.00 & 3.0083 & 953.33 & 3.9875 & 1071.67 & 4.1833 & 1190,00 & 4,3083 & 1308.33 & 4.3083 & 1426.67 & 4.3583 \\
\hline 836.67 & 3.8958 & 955.00 & 4.0000 & 1073.33 & 4.1833 & 1191.67 & 4.3042 & 1310.00 & 4.3083 & 1428.33 & 4.3833 \\
\hline 838.33 & 3.9042 & 956.67 & 4.0125 & 1075.00 & 4.1833 & 1193.33 & 4.2917 & 1311.67 & 4.3000 & 1430.00 & 4.3917 \\
\hline 840.00 & 3.8958 & 958.33 & 4.0000 & 1076.67 & 4.1833 & 1195.00 & 4.2750 & 1313.33 & 4.3083 & 1431.67 & 4.4000 \\
\hline 841.67 & 3.9125 & 960.00 & 39958 & 1078.33 & 4.1708 & 1196.67 & 4.2667 & 1315.00 & 4.3083 & 1433.33 & 4.3833 \\
\hline 843,33 & 3.9083 & 961.67 & 3.9958 & 1080.00 & 4.1667 & 1198.33 & 4.2708 & 1316.67 & 4.3042 & 1435.00 & 4.4000 \\
\hline 845.00 & 3.9042 & 963.33 & 4.0083 & 1081.67 & 4.1583 & 1200.00 & 4.2667 & 1318.33 & 4.2917 & 1436.67 & 4.4333 \\
\hline 846.67 & 3.8958 & 965.00 & 4.0083 & 1083.33 & 4.1625 & 1201.67 & 4.2583 & 1320.00 & 4.2833 & 1438.33 & 4.4625 \\
\hline 848.33 & 3.9042 & 968.67 & 4.0083 & 1085.00 & 4.1458 & 1203.33 & 4.2542 & 1321.67 & 4.3042 & 1440.00 & 4.4500 \\
\hline 850.00 & 3.3042 & 968.33 & 4.0167 & 1086.67 & 4.1458 & 1205.00 & 4.2750 & 1323.33 & 4.3125 & 1441,67 & 4.4167 \\
\hline 851.67 & 3.9167 & 970.00 & 4.0250 & 1088.33 & 4.1708 & 1206.67 & 4.2917 & 1325.00 & 4.3208 & 1443.33 & 4.3875 \\
\hline 853.33 & 3.9167 & 971.67 & 4,0250 & 1090.00 & 4.1833 & 1208.33 & 4.2917 & 1326.67 & 4.2917 & 1445.00 & 4.3833 \\
\hline 855.00 & 3.9250 & 973,33 & 4.0167 & 1091.67 & 4.1958 & 1210.00 & 4.2833 & 1328.33 & 4.2958 & 1446.67 & 4.4042 \\
\hline 856.67 & 3.9125 & 975.00 & 4.0125 & 1093.33 & 4.1833 & 1211.67 & 4.2833 & 1330.00 & 4.2875 & 1448.33 & 4.4083 \\
\hline 858.33 & 3.9083 & 976.67 & 4.0125 & 1095.00 & 4.1958 & 1213.33 & 4.2750 & 1331.67 & 4.2958 & 1450.00 & 4.4083 \\
\hline 860.00 & 3.9125 & 978.33 & 4.0250 & 1098.67 & 4.1917 & 1215.00 & 4.2833 & & 4.2875 & 1451.67 & 4.4125 \\
\hline 861.67 & 3.9167 & 980.00 & 4.0375 & 1098.33 & 4.1917 & 1216.67 & 4.2708 & 1335.00 & 4.2833 & 1453.33 & 4.4292 \\
\hline 263.33 & 3.9250 & 981.67 & 4.0458 & 1100.00 & & 1218.33 & 4.2750 & 133 & 4.2 & .00 & 4.4333 \\
\hline 865.00 & 3.9208 & 983.33 & 4.0375 & 1101.67 & 4.2208 & 12220.00 & 4.2583 & 1338.33 & 4.2792 & 1456.67 & 4.4000 \\
\hline 866.67 & 3.9375 & 985.00 & 4.0208 & 1103.33 & 4.2250 & 1221.67 & 4.2625 & & 4.2667 & 1458.33 & 4.3917 \\
\hline 868.33 & 3.9667 & 986.67 & 4.0250 & 1105.00 & 4.2250 & 1223.33 & 4.2583 & 1341.67 & 4.2667 & 1460.00 & 4.3833 \\
\hline 870.00 & 3.9833 & 988.33 & 4.0167 & 1106.67 & 4.2083 & 1225.00 & 4.2500 & 1343.33 & 4.2667 & 1461.67 & 4.4042 \\
\hline 871.67 & 3.9792 & 990.00 & 4.0500 & 1108.33 & 4.1958 & 1226.67 & 4.2458 & 1345.00 & 4.2917 & 1463.33 & 4.3958 \\
\hline 873.33 & 3.9583 & 991.67 & 4.0625 & 1110.00 & 4.1958 & 1228.33 & 4.2458 & 1346.67 & 4.3083 & 1465.00 & 4.4125 \\
\hline 875.00 & 3.9458 & 993.33 & 4.0833 & 1111,67 & 4.1917 & 1230.00 & 4.2583 & 1348.33 & 4.3042 & 1466.67 & 4.4083 \\
\hline 876.67 & 3.9458 & 995.00 & 4.0750 & 1113.33 & 4.1917 & 1231.67 & 4.2708 & 1350.00 & 4.2917 & 1468.33 & 4.4083 \\
\hline 878.33 & 3.9500 & 996.67 & 4.0867 & 1115.00 & 4.1792 & 1233.33 & 4.2833 & 1351,67 & 4.2875 & 1470.00 & 4.3958 \\
\hline 880.00 & 3.9625 & 998.33 & 4.0792 & 1116.67 & 4.1833 & 1235.00 & 4.2917 & 1353.33 & 4.2875 & 1471.67 & 4.3833 \\
\hline 881.67 & 3.9625 & 1000.00 & 4.0792 & 1118.33 & 4.1917 & 1236.67 & 4.2833 & 1355.00 & 4.2875 & 1473.33 & 4.3875 \\
\hline 883.33 & 3.9583 & 1001.67 & 4.0833 & 1120.00 & 4.1917 & 1238.33 & 4.2958 & 1356.67 & 4.2750 & 1475.00 & 4.3917 \\
\hline 885.00 & 3.9542 & 1003.33 & 4.0750 & 1121.67 & 4.1875 & 1240.00 & 4.2917 & 1358.33 & 4.2958 & 1476.67 & 4.4042 \\
\hline 886,67 & 3.9500 & 1005.00 & 4.0875 & 1123.33 & 4.2000 & 1241.67 & 4.2875 & 1360.00 & 4.3125 & 1478.33 & 4.4083 \\
\hline 898,33 & 3.9542 & 1006.67 & 4.0875 & 5125.00 & 4.2250 & 1243.33 & 4.2708 & 1361.67 & 4,3167 & 1480.00 & 4.4125 \\
\hline 89000 & 3.9542 & 1008.33 & 4.0958 & 1126.67 & 4.2417 & 1245.00 & 4.2708 & 1363.33 & 4.3042 & 1481.67 & 4.3917 \\
\hline 891,67 & 3.9625 & 1010.00 & 4.0833 & 1128.33 & 4.2333 & 1246.67 & 4.2792 & 1365.00 & 4.3000 & 1483.33 & 4.3917 \\
\hline
\end{tabular}


Table C16. Thermo-Gravimetric Analys is Data for $K$ Basin Fuel Sample Run 51 at $69^{\circ} \mathrm{C}$. (Page 3 of 7)

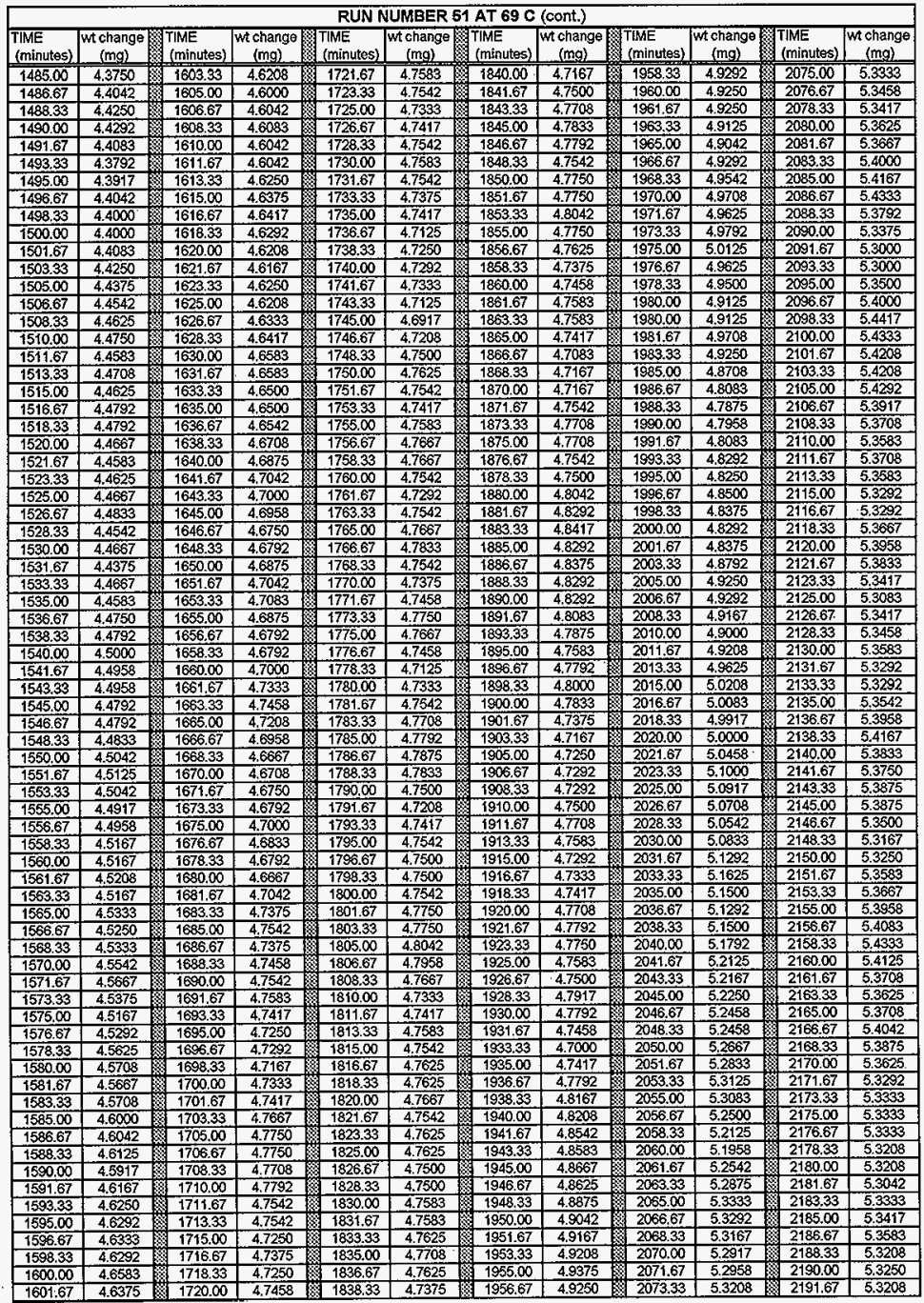


Table C16. Thermo-Gravimetric Analysis Data for $K$ Basin Fuel Sample Run 51 at $69^{\circ} \mathrm{C}$. (Page 4 of 7 )

\begin{tabular}{|c|c|c|c|c|c|c|c|c|c|c|c|}
\hline \multicolumn{12}{|c|}{ RUN NUMBER 61 AT $69 \mathrm{C}$ (cont.) } \\
\hline $\begin{array}{l}\text { TIME } \\
\text { (minutes) }\end{array}$ & $\begin{array}{c}\text { wh change } \\
\text { (mg) }\end{array}$ & $\begin{array}{l}\text { TiME } \\
\text { (minutes) }\end{array}$ & $\begin{array}{c}\text { we change } \\
\text { (mg) }\end{array}$ & $\begin{array}{l}\text { TiME } \\
\text { (minutes) }\end{array}$ & $\begin{array}{c}\text { wt change } \\
\text { (mg) }\end{array}$ & $\begin{array}{l}\text { TiME } \\
\text { (minutes) }\end{array}$ & \begin{tabular}{c|} 
wt change \\
(mg)
\end{tabular} & $\begin{array}{l}\text { TIME } \\
\text { (minutes) } \\
\end{array}$ & \begin{tabular}{|c|} 
Wh changs \\
(mg)
\end{tabular} & $\begin{array}{l}\text { TimE } \\
\text { (minutes) }\end{array}$ & $\begin{array}{c}\text { Wt change } \\
\text { (mg) }\end{array}$ \\
\hline 2993.33 & 5.3583 & 2311.67 & 5.4875 & 2430.00 & 5.5333 & 2548.33 & 5.6750 & 2666.67 & 5.7333 & 2785.00 & 5.9000 \\
\hline 2195.00 & 5.3708 & 2313.33 & 5.4833 & 2431.67 & 5.5667 & 2550.00 & 5.6667 & 2688.33 & 5.7375 & 2786.67 & 5.9083 \\
\hline 2196.67 & & & 4.450 & & & 2551.67 & & 2670.00 & 57250 & 2788.33 & 5.9208 \\
\hline 2198.33 & 1167 & 2316.67 & 5.4833 & 2435.00 & 5.5417 & 2553.33 & 5.6708 & 2671.67 & 5.7458 & 2790,00 & 5.9083 \\
\hline 2200.00 & & 2318.33 & 5.4792 & 2436,67 & 5.5375 & 2555,00 & & 2673.33 & 5.7625 & 2791.67 & 5.9083 \\
\hline 2201.67 & 5.4333 & 2320.00 & 5.4667 & 2438.33 & 5.5292 & 2556.67 & 5.6708 & 2675.00 & 5.7667 & 2793.33 & 5.9250 \\
\hline 2203.33 & & 2321.67 & 5.4458 & & & 2558.33 & & 2676.67 & & 2795.00 & \\
\hline 2205.00 & 4667 & 2323.33 & 5.4667 & 2441.67 & 5.5417 & 2560.00 & 5.6667 & 2678.33 & 5.7458 & 2796.67 & 5.9292 \\
\hline 2206.67 & 6667 & 2325.00 & 5.4750 & 2443.33 & 5.5542 & 2561.67 & 5.6417 & 2680.00 & 5.7500 & 2798.33 & 5.9292 \\
\hline 2208.33 & 5.4208 & 2326.67 & 5.4958 & 2445.00 & 5.5500 & 2563.33 & 5.6500 & 2681.67 & 5.7667 & 2800,00 & 5.9250 \\
\hline 2210.00 & & $\frac{102011}{2328.33}$ & & 2446.67 & & 2565.00 & & 2683.33 & $5=7$ & 2801,67 & 5.9292 \\
\hline 2211.67 & 3417 & 2330.00 & 5.4667 & 2448.33 & 5.5417 & 2566.67 & 5.6833 & 2685.00 & 5.7875 & 2803.33 & 5.9208 \\
\hline 2213.33 & & 2331.67 & 5.4750 & 2450.00 & & 2588.33 & & 2606.67 & 7917 & & \\
\hline 2215.00 & 1458 & 2333.33 & 5.4750 & 2451.67 & 5.5252 & 2570.00 & 5.6875 & 2688.33 & 5.7958 & 2806.67 & 5.9458 \\
\hline 2216.67 & & 2335.00 & sons & & 5.5333 & 2571.67 & & 2690.00 & 5.7792 & & \\
\hline 2218.33 & 5.4250 & 2336.67 & 5.4875 & 2455.00 & 5.5375 & 2573.33 & 5.7250 & 2691.67 & 5.7625 & 2810.00 & 5.9500 \\
\hline 2220.00 & & 2338.33 & 5.4792 & 2456.67 & 5.5250 & 2575.00 & 5.7125 & 2693.33 & 5.7583 & 2811.67 & 5.9500 \\
\hline 2221.67 & 5.4000 & 2340.00 & 5.4750 & 2458.33 & 5.5250 & 2576.67 & 5.7042 & 2695.00 & 5.7792 & 2813.33 & 5.9583 \\
\hline 2223.33 & & & 5.4500 & 2460.00 & 5.5500 & 2578.33 & 5.6875 & 2686.67 & & & 5.9750 \\
\hline 2225.00 & 5.4208 & 2343.33 & 5.4458 & 2461.67 & 5.5792 & 2580.00 & 5.6792 & 2688.33 & 5.8125 & 2816.67 & 5.9792 \\
\hline 2226.67 & & 2345.00 & 5.4250 & 2463.33 & 5.5917 & 2581.67 & 5.6750 & 2700.00 & 5.8042 & & 5.9708 \\
\hline 2228.33 & 5.4000 & 2346.67 & 5.4417 & 2465.00 & 5.5750 & 2583.33 & 5.6708 & 2701.67 & 5.7875 & 2820.00 & 5.9750 \\
\hline 2230.00 & & 2348.33 & 5.4458 & 2466.67 & 5.5750 & 2585.00 & 5.6792 & 2703.33 & 5.7792 & 2821.67 & 5.9708 \\
\hline 2231.67 & 5.4125 & 2350.00 & 5.4792 & 2468.33 & 5.5917 & 2586.67 & 5.6875 & 2705.00 & 5.7583 & 2823.33 & 5.9667 \\
\hline 2233.33 & & 2351.67 & 5.4750 & 2470.00 & 5.6167 & 2588.33 & 5.6875 & 2706.67 & 5.7750 & 2825.00 & 5.9417 \\
\hline 2235.00 & 667 & 2353.33 & 5.4958 & 2471.67 & 5.6167 & 2590.00 & 5.6750 & 2708.33 & 5.7917 & 2826.67 & 5.9625 \\
\hline 2236.67 & & 2355.00 & & 2473.33 & 5.6 & 2591.67 & 5.6667 & 2710.00 & 5.8083 & 2828.33 & 5.9792 \\
\hline 2238.33 & 3 & 2356.67 & 5.5375 & 2475.00 & 5.6042 & 2593.33 & 5.6833 & 2711.67 & 5.8042 & 2830.00 & 6.0042 \\
\hline 2240.00 & & 2358.33 & 5.5292 & 2476.67 & 5.5958 & 2595.00 & 5.7125 & 2713.33 & 5.8083 & 2831.67 & 5.9958 \\
\hline 2241.67 & & 2360.00 & 7 & 2478.33 & 5.6000 & 2596.67 & 5.7208 & 2715.00 & 5.8167 & 2833.33 & 5.9917 \\
\hline 2243.33 & & 2361.67 & $5.5 \mathrm{C}$ & 2480.00 & 5.5833 & 2598.33 & 5.7125 & 2716.67 & $\frac{5.8}{5.8}$ & 2835.00 & 6.0083 \\
\hline 2245.00 & $\frac{25}{667}$ & 2363.33 & 5.5000 & $248\} .67$ & 5.5875 & 2600.00 & 5.7083 & 2718.33 & 5.8125 & 2836.67 & 6.0125 \\
\hline 2246.67 & & 2365.00 & & & & & 42 & 2720.00 & 5.8 & 2838.33 & 6.0125 \\
\hline 2248.33 & & 2386.67 & 8 & & & & 5.7167 & 2721.67 & 5.8208 & 2840.00 & 6.0083 \\
\hline 2250.00 & 375 & 2368.33 & 5.5000 & 2486.67 & & 260 & 5.7167 & 2723.33 & 5.8292 & 2841.67 & 6.0083 \\
\hline 2251.67 & & 2370.00 & & & & & 5.7 & 2725.00 & & 2843.33 & 6.0000 \\
\hline 2253.33 & & 67 & & & & & 57 & 2726.67 & 5.8 & 2845.00 & 5.9792 \\
\hline 2255.00 & & 2373.33 & & 67 & & & & 2728.33 & & 2846.67 & 5.9958 \\
\hline 2256.67 & & & & & & & & & 5.8083 & 2848.33 & 6.0083 \\
\hline 2258.33 & & & & & & & & & & & 0125 \\
\hline 2260.00 & 5.40 & 237 & & 67 & & & $5=0$ & & & 2851.67 & .0042 \\
\hline 2261.67 & & & & & & & & & & 2853.33 & 6.0125 \\
\hline 2263.33 & & 2381.67 & & & & & & 2736.67 & & 2855.00 & 5.0208 \\
\hline 2265.00 & & & & & & & & & & 67. & 67 \\
\hline 2266.67 & & 2365.00 & & & $\frac{2 .}{5 .}$ & & 2 & & & 2856.33 & 6.0167 \\
\hline 2268.33 & & 23 & & & & & & & & & \\
\hline & & & & & & & & & & 2861.67 & 50 \\
\hline 2271. & & 2390 & & & & & & & & & \\
\hline 2273.33 & & & & & & & & & & .00 & 6.0042 \\
\hline 2275.00 & & & & & & 263 & & & & 2866.67 & 3.0042 \\
\hline 2276.67 & & 239 & & & & & & & & & \\
\hline 2278.33 & & & & & & & & 275 & 5.8 & 2870.00 & 6.0292 \\
\hline 2280 & & & & & & & & & & & .0417 \\
\hline 2281.67 & & & & & & & & & & & 33 \\
\hline 2283.33 & & & & & & & & & & & 500 \\
\hline 2285.00 & & & & & & & & & & & 6.0500 \\
\hline 2286.67 & & & & & & & & & & & 1583 \\
\hline 2288.33 & & 2406.67 & & 00 & & & & & & & \\
\hline 2290.00 & & & & & & & & & & & 25 \\
\hline 229 & & & & & & & & .00 & & & 37 \\
\hline 2293.33 & & & & & & & & & & & \\
\hline 22 & & & & & & & & & & & \\
\hline \begin{tabular}{|l|}
2296.67 \\
\end{tabular} & 5. & 2415.00 & & & & & & & & 2888.33 & 6.0458 \\
\hline 2298.33 & & & & & & & & 277 & & & 6.0458 \\
\hline 2300.00 & & & & 253 & & 2655,00 & & & & & 375 \\
\hline 2301.67 & 5.5125 & 2420.00 & 5.5203 & 253 & & & & & & 289 & 6.0208 \\
\hline 2303.33 & & 2421.67 & 5.5292 & 2540.00 & 5.6667 & 2658.33 & 5.7 & 277 & & 2895.00 & 6.0125 \\
\hline 2305.00 & 5.5000 & 242 & 5.54 & & & 26 & & & & 2896.67 & \\
\hline 2306.67 & & & & & & 2661.67 & & 2780.00 & & 2898.33 & 6.0125 \\
\hline 2308.33 & 5.4875 & 2426.67 & 5.5 & 2545.00 & 5.67 & 2663.33 & & 2781.67 & & 290 & 6.0167 \\
\hline & 5.4958 & 2428.33 & 5.5083 & 2546.67 & 5.6917 & 2665.00 & 5.7333 & 2783.33 & 5.9000 & 2901.67 & 6.0167 \\
\hline
\end{tabular}


Table C16. Thermo-Gravimetric Analysis Data for $K$ Basin Fuel Sample Run 51 at $69{ }^{\circ} \mathrm{C}$. (Page 5 of 7)

\begin{tabular}{|c|c|c|c|c|c|c|c|c|c|c|c|c|c|}
\hline \multicolumn{12}{|c|}{ RUN NUMBER 51 AT $69 \mathrm{C}$ (cont.) } & \multicolumn{2}{|l|}{$\therefore$} \\
\hline $\begin{array}{l}\text { TIME } \\
\text { (minutes) }\end{array}$ & $\begin{array}{c}\text { Wh change } \\
\text { (mg) }\end{array}$ & $\begin{array}{l}\text { TIME } \\
\text { (minutes) }\end{array}$ & $\begin{array}{c}\begin{array}{c}\text { Wh change } \\
(\mathrm{mg})\end{array} \\
\end{array}$ & & $\begin{array}{l}\text { TIME } \\
\text { (minutes) }\end{array}$ & $\begin{array}{c}\text { wh change } \\
\text { (mg) }\end{array}$ & & $\begin{array}{l}\text { TIME } \\
\text { (minutes) }\end{array}$ & $\begin{array}{c}\text { We chango } \\
\text { (mg) }\end{array}$ & $\begin{array}{l}\text { TiME } \\
\text { (minutes) }\end{array}$ & $\begin{array}{c}\text { wt change } \\
\text { (ma) }\end{array}$ & $\begin{array}{l}\text { TIME } \\
\text { (minutes) }\end{array}$ & $\begin{array}{c}\text { wh change } \\
\text { (mg) }\end{array}$ \\
\hline 2903.33 & 6.0167 & 3021.67 & 6.1875 & & 3140.00 & 6.2917 & & 3258.33 & 6.4167 & 3376.67 & 6.8167 & 3495.00 & 6.9417 \\
\hline 2905.00 & 6.0125 & 3023.33 & 6.1875 & & 3141.67 & 6.2875 & & 3260.00 & 6,4167 & 3378.33 & 6.8125 & 3496.67 & 6.9458 \\
\hline 2906.67 & 6.0125 & 3025.00 & 6.1875 & & 3143.33 & 6.2917 & & 3261.67 & 6.4292 & 3380.00 & 6.8208 & 3498.33 & 6.9458 \\
\hline 2908.33 & 6.0125 & 3026.67 & 6.1875 & & 3145.00 & 6.2958 & & 3263.33 & 6,4333 & 3381.67 & 6.8208 & 3500.00 & 6.9500 \\
\hline 2910.00 & 6.0208 & 3028.33 & 6.1958 & & 3146.67 & 6.3042 & & 3265.00 & 6.4500 & 3383.33 & 6.8208 & 3501.67 & 6.9500 \\
\hline 2911.67 & 6.0333 & 3030.00 & 6.1958 & & 3148.33 & 6.3083 & & 3266.67 & 6.4542 & 3335.00 & 6.8125 & 3503.33 & 6.9542 \\
\hline 2913.33 & 6.0375 & 3031.67 & 6.2042 & & 3150.00 & 6.3083 & & 3268,33 & 6.4583 & 3386,67 & 6.8208 & 3505.00 & 6.9500 \\
\hline 2915,00 & 6.0292 & 3033.33 & 6.1958 & & 3151.67 & 6.3000 & & 3270.00 & 6.4542 & 3388.33 & 6.8208 & 3506.67 & 6.9500 \\
\hline 2916.67 & 6.0250 & 3035.00 & 6.2042 & & 3153.33 & 6.2917 & & 3271.67 & 6.4542 & 3390.00 & 6.8202 & 3508.33 & 6.9458 \\
\hline 2918.33 & 6.0292 & 3036.67 & 6.2042 & & 3155.00 & 6.2958 & & 3273,33 & 6.4667 & 3391.67 & 6.8292 & 3510.00 & 6.9500 \\
\hline 2920.00 & 6.0417 & 3038.33 & 6.2083 & & 3156.67 & 6.3000 & & 3275,00 & 6.4833 & 3393.33 & 6.8417 & 3511.67 & 6.9542 \\
\hline 2921.67 & 6.0458 & 3040.00 & 6.2000 & & 3158.33 & 6.3083 & & 3276.67 & 6.5000 & 3395.00 & 6.8500 & 3513.33 & 6.9667 \\
\hline 2923.33 & 6.0500 & 3041.67 & 6.1958 & & 3160.00 & 6.3000 & & 3278,33 & 6.5042 & 3396.67 & 6.8500 & 3515,00 & 6.9708 \\
\hline 2925.00 & 6.0500 & 3043.33 & 6.1958 & & 3161.67 & 6.2958 & & 3280.00 & 6.5125 & 3398.33 & 6.8500 & 3516.67 & 6.9792 \\
\hline 2926.67 & 6.0542 & 3045.00 & 6.2042 & & 3163.33 & 6.2875 & & 3281.67 & 6.5250 & 3400.00 & 6.8500 & 3518.33 & 6.9792 \\
\hline 2928.33 & 6.0525 & 3046.67 & 6.2083 & & 3165.00 & 6.2917 & & 3283.33 & 6.5417 & 3401.67 & 6.8583 & 3520.00 & 6.9750 \\
\hline 2930.00 & 6.0708 & 3048.33 & 6.2125 & & 3166.67 & 6.2917 & & 3285.00 & 6.5500 & 3403.33 & 6.8625 & 3521.67 & 6.9708 \\
\hline 2931.67 & 6.0667 & 3050.00 & 6.2167 & & 3168.33 & 6.3000 & & 3286.67 & 6.5500 & 3405.00 & 6.8625 & 3523.33 & 6.9708 \\
\hline 2933.33 & 6.0667 & 3051.67 & 6.2167 & & 3170.00 & 6.2958 & & 3288,33 & 6.5625 & 3406.67 & 6.8667 & 3525.00 & 6.9792 \\
\hline 2935.00 & 6.0625 & 3053,33 & 6.2250 & & 3171.67 & 6.2958 & & 3290.00 & 6.5708 & 3408.33 & 6.8625 & 3526.67 & 6.9833 \\
\hline 2936.67 & 6.0667 & 3055.00 & 6.2292 & & 3173.33 & 6.2917 & & 3291.67 & 6.5750 & 3410.00 & 6.8625 & 3528.33 & 6.9958 \\
\hline 2938.33 & 6.0667 & 3056.67 & 6.2375 & & 3175.00 & 6.2917 & & 3293.33 & 6.5708 & 3411.67 & 6.8625 & 3530.00 & 7,0000 \\
\hline 2940.00 & 6.0708 & 3058.33 & 6.2417 & & 3176.67 & 6.2875 & & 3225.00 & 6.5792 & 341333 & 6.8667 & 3531.67 & 7.0083 \\
\hline 2941.67 & 6.0750 & 3000.00 & 6.2417 & & 3178.33 & 6.2833 & & 3296.67 & 6.5760 & 3415.00 & 6.8667 & 3533.33 & 7,0125 \\
\hline 2943.33 & 6.0875 & 3051.67 & 6.2375 & & 3180.00 & 6.2833 & & 3298.33 & 6.5792 & 3416.67 & 6.8667 & 3535,00 & 7.0208 \\
\hline 2945.00 & 6.0875 & 3063.33 & 6.2333 & & 318167 & 6.2875 & & 3300.00 & 6.5792 & 3418.33 & 6.8708 & 3536.67 & 7.0250 \\
\hline 2946.67 & 6.1042 & 3065.00 & 6.2375 & & 3183.33 & 6.2875 & & 3301.67 & 6.5917 & 3420.00 & 6.8750 & 3538.33 & 7,0292 \\
\hline 2948.33 & 6.1083 & 3066.67 & 6.2417 & & 3185.00 & 6.2917 & & 3303,33 & 6.5958 & 3421,67 & 6.8750 & 3540.00 & 7.0292 \\
\hline 2950.00 & 6.1167 & 3068.33 & 6.2375 & & 3186.67 & 6.2958 & & 3305.00 & 6.6042 & 3423.33 & 6.8750 & 3541.67 & 7.0292 \\
\hline 2951.67 & 6.1125 & 3070.00 & 6.2375 & & 3188.33 & 6.2958 & & 3306.67 & 6.6167 & 3425,00 & 6.8792 & 3543.33 & 7.0250 \\
\hline 2953,33 & 6.1083 & 3071.67 & 6.2417 & & 3190,00 & 6.2917 & & 3308,33 & 6.6292 & 3426.67 & 6.8792 & 3545.00 & 7.0375 \\
\hline 2955.00 & 6.1167 & 3073.33 & 6.2500 & & 3191.67 & 6.2875 & & 3310,00 & 6.6333 & 3428.33 & 6.8708 & 3546.67 & 7.0500 \\
\hline 2956.67 & 6.1167 & 3075.00 & 6.2542 & & 3193.33 & 6.2875 & & 3311.67 & 6.6375 & 3430.00 & 6.8667 & 3548.33 & 7.0583 \\
\hline 2958.33 & 6.1250 & 3076.67 & 6.2542 & & 3195.00 & 6.2875 & & 3313.33 & 6.6375 & 3431.67 & 6.8667 & 3550.00 & 7.0500 \\
\hline 2960.00 & 6.1208 & 3078.33 & 6.2542 & & 3196.67 & 6.2917 & & 3315.00 & 6.6417 & 3433.33 & 6.8750 & 3551.67 & 7.0458 \\
\hline 2961.67 & 6.1292 & 3080.00 & 6.2542 & & 3198.33 & 6.2958 & & 3316.67 & 6.6458 & 3435.00 & 6.8750 & 3553.33 & 7.0458 \\
\hline 2963,33 & 6.1292 & 3081.67 & 6.2542 & & 3200,00 & 6.3000 & & 3318.33 & 6.6542 & 3436.67 & 6.8792 & 3555.00 & 7.0500 \\
\hline 2965.00 & 6.1375 & 3083.33 & 6.2583 & & 3201.67 & 6.3042 & & 3320.00 & 6.6533 & 3438.33 & 6.8792 & 3556.67 & 7.0458 \\
\hline 2966.67 & 6.1375 & 3085.00 & 6.2583 & & 3203.33 & 6.3042 & & 3321,67 & 6.6708 & 3440,00 & 6.8792 & 3558.33 & 7.0417 \\
\hline 2968,33 & 6.1375 & 3086.67 & 6.2625 & & 3205.00 & 6.3000 & & 3323,33 & 6.6750 & 3441.67 & 6.8833 & 3560.00 & 7.0417 \\
\hline 2970.00 & 6.1417 & 3088.33 & 6.2583 & & 3206.67 & 6.2875 & & 3325.00 & 6.6750 & 3443,33 & 6.8833 & 3561.67 & 7.0458 \\
\hline 2971.67 & 6.1458 & 3090,00 & 6.2500 & & 3208.33 & 6.2833 & & 3326,67 & 6.6750 & 3445,00 & 6.8833 & 3563.33 & 7.0500 \\
\hline 2973.33 & 6.1500 & 3091.67 & 6.2542 & & 3210.00 & 6.2833 & & 3328.33 & 6.6750 & 3446.67 & 6.8833 & 3565.00 & 7.0525 \\
\hline 2975,00 & 6.1500 & 3093.33 & 6.2625 & & 3211.67 & 6.2875 & & 3330.00 & 6.6833 & 3448.33 & 6.8917 & 3566.67 & 7.0625 \\
\hline 2976,67 & 6.1500 & 3095.00 & 6.2750 & & 3213.33 & 6.2875 & & 3331.67 & 6.6875 & 3450.00 & 6.9000 & 3568.33 & 7.0625 \\
\hline 2978.33 & 6.1500 & 3096.67 & 6.2708 & & 3215.00 & 6.3000 & & 3333.33 & 6.7042 & 3451.67 & 6.9000 & 357000 & 7.0458 \\
\hline 2980.00 & 6.1500 & 3098.33 & 6.2708 & & 3216.67 & 6.2625 & & 3335.00 & 6.7083 & 3453.33 & 6.9042 & 3571.67 & 7.0458 \\
\hline 2981,67 & 6.1583 & 3100.00 & 6.2750 & & 3218.33 & 6.2000 & & 3336.67 & 6.7167 & 3455,00 & 6.9083 & 3573,33 & 7.0458 \\
\hline 2983.33 & 6.1583 & 3101.67 & 6.2792 & & 322 & 50 & & 3338,33 & 6.7125 & 3456.67 & 6.9125 & 3575.00 & 7.0500 \\
\hline 2985,00 & 6.1667 & 3103.33 & 6.2792 & & 3221,67 & 6.0917 & & 3340.00 & 6.7208 & 3458.33 & 6.9083 & 3576.67 & 7.0542 \\
\hline 2986.67 & 6.1625 & 3105.00 & 62750 & & 3223.33 & 6.0833 & & 3341.67 & 6.7208 & 3460.00 & 6.9083 & 3578.33 & 7.0583 \\
\hline 2988.33 & 6.1625 & 3106.67 & 6,2760 & & 3225,00 & 6.1208 & & 3343.33 & 6.7292 & 3461,67 & 6.9083 & 3580.00 & 7.0583 \\
\hline 2990.00 & 6.1542 & 3108.33 & 6.2750 & & 3226.67 & 6.1708 & & 3345.00 & 6.7417 & 3463.33 & 6.9208 & 3581.67 & 7.0542 \\
\hline 2991.67 & 6.1583 & 3110.00 & 6.2750 & & 3228.33 & 6.2333 & & 3346.67 & 6.7458 & 3465.00 & 6.9208 & 3583.33 & 7.0583 \\
\hline 2993.33 & 6.1625 & 3111.67 & 6.2792 & & 3230.00 & 6.2667 & & 3348.33 & 6.7458 & 3466.67 & 6.9250 & 3585.00 & 7.0583 \\
\hline 2995.00 & 6.1708 & 3113.33 & 6.2792 & & 3231.67 & 6.2958 & & 3350.00 & 6.7417 & 3468.33 & 6.9208 & 3586.67 & 7,0667 \\
\hline 2996.67 & 6.1750 & 3116.00 & 6.2792 & & 3233.33 & 6.3167 & & 3351.67 & 6.7625 & 3470.00 & 6.9250 & 3588.33 & 7.0667 \\
\hline 2998.33 & 6.1792 & 3116.67 & 6.2708 & & 3235.00 & 6.3292 & & 3353.33 & 6.7708 & 3471.67 & 6.9292 & 3590.00 & 7.0708 \\
\hline 300000 & 6.1792 & 3118.33 & 6.2750 & & 3236.67 & 6.3333 & & 3355.00 & 6.7750 & 3473.33 & 6.9292 & 3591.67 & 7.0667 \\
\hline 3001.67 & 6.1750 & 3120.00 & 6.2792 & & 3238.33 & 6.3375 & & 3356.67 & 6.7625 & 3475.00 & 6.9292 & 359333 & 7.0667 \\
\hline 3003.33 & 6.1750 & 3121.67 & 6.2875 & & 3240.00 & 6.3458 & & 3358.33 & 6.7625 & 3476.67 & 6.9292 & 3595.00 & 7.0750 \\
\hline 3005.00 & 6.1750 & 3123.33 & 6.2875 & & 3241.67 & 6.3625 & & 3360.00 & 6.7750 & 3478.33 & 6.9250 & 3556.67 & 7.0833 \\
\hline 300667 & 6.1792 & 3125,00 & 6.2833 & & 3243,33 & 6.3667 & & 3361.67 & 6.7875 & 3480.00 & 6.9292 & 3598.33 & 7.0833 \\
\hline 3008.33 & 6.1833 & 3126.67 & 6.2833 & & 3245.00 & 6.3708 & & 3363.33 & 6.7958 & 3481,67 & 6.9333 & 3600.00 & 7.0875 \\
\hline 3010.00 & 6.1875 & 3128.33 & 6.2233 & & 3246.67 & 6.3792 & & 3355.00 & 6.7917 & 3483.33 & 6.9417 & 3601.67 & 7.0875 \\
\hline 3011.67 & 6.1917 & 3130.00 & 6.2958 & & 3248.33 & 6.3917 & & 3366.67 & 6.7917 & 3485.00 & 6.9600 & 3603.33 & 7.0917 \\
\hline 3013.33 & 6.1917 & 3131.67 & 6.2958 & & 3250,00 & 6.4000 & & 3368.33 & 6.7958 & 3486.67 & 6.9500 & 3605.00 & 7.0917 \\
\hline 3015,00 & 6.1917 & 3133.33 & 6.3000 & & 3251.67 & 6.4083 & & 3370.00 & 6.8000 & 3483.33 & 6.9458 & 3606.67 & 7.0953 \\
\hline 3016.67 & 6.1833 & 3135.00 & 6.2958 & & 3253.33 & 6.4208 & & 3371.67 & 6.8125 & 3490.00 & 6.9375 & 3608.33 & 7.1042 \\
\hline 3018.33 & 6.1833 & 3136.67 & 6.3000 & & 3255,00 & 6.4208 & & 3373.33 & 6.8208 & 3491.67 & 6.9333 & 3610.00 & 7.1083 \\
\hline 3020.00 & 6.1833 & 3138.33 & 6.2958 & & 3256.67 & 6.4167 & & 3375.00 & 6.8250 & 3493.33 & 6.9375 & 3611.67 & 7.1083 \\
\hline
\end{tabular}


Table C16. Thermo-Gravimetric Analysis Data for $\mathrm{K}$ Basin Fuel Sample Run 51 at $69^{\circ} \mathrm{C}$. (Page 6 of 7)

\begin{tabular}{|c|c|c|c|c|c|c|c|c|c|c|c|}
\hline \multicolumn{12}{|c|}{ RUN NUMBER 51 AT $69 \mathrm{C}$ (cont.) } \\
\hline $\begin{array}{l}T M E \\
\text { (minutes) }\end{array}$ & $\begin{array}{c}\text { wt change } \\
\text { (mg) }\end{array}$ & $\begin{array}{l}\text { Time } \\
\text { (minutes) }\end{array}$ & $\begin{array}{c}\text { Wt change } \\
\text { (mg) }\end{array}$ & $\begin{array}{l}\text { TiMg } \\
\text { (minutes) }\end{array}$ & $\begin{array}{c}\text { wt change } \\
\text { (mg) }\end{array}$ & $\begin{array}{l}\text { TIME } \\
\text { (minutes) }\end{array}$ & $\begin{array}{c}\text { we change } \\
\text { (mg) }\end{array}$ & $\begin{array}{l}\text { TIME } \\
\text { (minutes) }\end{array}$ & $\begin{array}{c}\text { wt change } \\
\text { (mg) }\end{array}$ & \begin{tabular}{|l|} 
Time \\
(minutes)
\end{tabular} & \begin{tabular}{|c|}
$\begin{array}{c}\text { wt change } \\
\text { (mg) }\end{array}$ \\
\end{tabular} \\
\hline 3613.33 & 7.1042 & 3731.67 & 7.1833 & 3850.00 & 7.4333 & 3968.33 & 7.5000 & 4086.67 & 7.5167 & 4205.00 & 7.4875 \\
\hline 3615.00 & 7.1042 & 3733.33 & 7.1875 & 3851.67 & 7.4333 & 3970.00 & 7.5083 & 4088.33 & 7.5125 & 4206.67 & 7.4875 \\
\hline 3616.67 & 7.1000 & 3735.00 & 7.1833 & 3853.33 & 7.4333 & 3971,67 & 7.5083 & 4090.00 & 7.5208 & 4208.33 & 7.5000 \\
\hline 3618.33 & 7.1000 & 3736.67 & 7.1875 & 3355.00 & 7.4458 & 3973.33 & 7.5125 & 4091,67 & 7.5250 & 4210,00 & 7.5042 \\
\hline 3620.00 & 7.1083 & 3738.33 & 7.1958 & 3856.67 & 7.4458 & 3975.00 & 7.5042 & 4093.33 & 7.5292 & 4211.67 & 7.5083 \\
\hline .3621 .67 & 7.1167 & 3740.00 & 7.2083 & 3858.33 & 7.4583 & 3976,67 & 7.5042 & 4095.00 & 7.5333 & 4213,33 & 7,5042 \\
\hline 3623.33 & 7.1167 & 3741.67 & 7.2125 & 3860.00 & 7.4583 & 3978.33 & 7.5000 & 4096.67 & 7.5250 & 4215.00 & 7.5000 \\
\hline 3625.00 & 7.1042 & 3743.33 & 7.2208 & 3867.67 & 7.4583 & 3980.00 & 7.5000 & 4098.33 & 7.5292 & 4216,67 & 7.5000 \\
\hline 3626.67 & 7.1042 & 3745.00 & 7.2333 & 3863.33 & 7.4625 & 3981.67 & 7.5042 & 4100.00 & 7.5292 & 4218.33 & 7.4958 \\
\hline 3628.33 & 7.1125 & 3746.67 & 7.2458 & 3865.00 & 7.4625 & 3883.33 & 7.5083 & 4101.67 & 7.5333 & 4220.00 & 7.5042 \\
\hline 3630.00 & 7.1250 & 3748.33 & 7.2500 & 3865.67 & 7.4708 & 3985.00 & 7.5083 & 4103.33 & 7.5333 & 4221.67 & 7.5125 \\
\hline 3631.67 & 7.1375 & 3750.00 & 7.2500 & 3868.33 & 7.4708 & 3986.67 & 7.5042 & 4105,00 & 7.5292 & 4223.33 & 7.5250 \\
\hline 3633.33 & 7.1500 & 3751.67 & 7.2625 & 3870.00 & 7.4708 & 3988.33 & 7.5000 & 4106.67 & 7.5292 & 4225.00 & 7.5250 \\
\hline 3635.00 & 7.1583 & 3753.33 & 7.2708 & 3871.67 & 7.4708 & 3990.00 & 7.4958 & 4108.33 & 7.5250 & 4226.67 & 7.5292 \\
\hline 3636.67 & 7.1583 & 3755.00 & 7.2792 & 3873.33 & 7.4792 & 3991.67 & 7.5000 & 4110.00 & 7.5167 & 4228.33 & 7.5333 \\
\hline 3638.33 & 7.1542 & 3756.67 & 7.2792 & 3875.00 & 7.4792 & 3993.33 & 7.5000 & 4111.67 & 7.5208 & 4230.00 & 7.5417 \\
\hline 3640.00 & 7.1458 & 3758.33 & 7.2833 & 3376.67 & 7.4750 & 3995.00 & 7.5042 & 4113.33 & 7.5125 & 4231.67 & 7.5500 \\
\hline 3641.67 & 7.1542 & 3760.00 & 7.2833 & 3878.33 & 7.4625 & 3996.67 & 7.5042 & 4115.00 & 7.5208 & 4233.33 & 7,5542 \\
\hline 3643.33 & 7.1542 & 3761.67 & 7.2917 & 3680.00 & 7.4667 & 3998.33 & 7.5042 & 4116.67 & 7.5125 & 4235.00 & 7.5525 \\
\hline 3645.00 & 7.1625 & 3763.33 & 7.2917 & 3881.67 & 7.4750 & 4000.00 & 7.5083 & 4118.33 & 7.5167 & 4236.67 & 7.5525 \\
\hline 3546.67 & 7.1542 & 3765.00 & 7.2958 & 3883.33 & 7.4792 & 4001.67 & 7.5042 & 4120.00 & 7.5167 & 4238.33 & 7,5792 \\
\hline 3648.33 & 7.1542 & 3766.67 & 7.2917 & 3885.00 & 7.4833 & 4003.33 & 7.5042 & 4121.67 & 7.5167 & 4240.00 & 7.5750 \\
\hline 3650.00 & 7.1583 & 3768.33 & 7.3042 & 3885.67 & 7.4875 & 4005.00 & 7.5000 & 4123.33 & 7.5208 & 4241.67 & 7.5750 \\
\hline 3651.67 & 7,1583 & 3770.00 & 7.3167 & 3888.33 & 7.4875 & 4006.67 & 7.5000 & 4125.00 & 7.5250 & 4243.33 & 7.5708 \\
\hline 3653.33 & 7.1583 & 3771.67 & 7.3250 & 3890.00 & 7.4917 & 4008.33 & 7.5000 & 4126.67 & 7.5292 & 4245.00 & 7.5760 \\
\hline 3655.00 & 7.1500 & 3773.33 & 7.3250 & 3691.67 & 7.4875 & 4010.00 & 7.5000 & 4128.33 & 7.5250 & 4246.67 & 7.5792 \\
\hline 3656.67 & 7.1500 & 3775.00 & 7.3333 & 3693.33 & 7.4917 & 4011.67 & 7.4958 & 4130.00 & 7.5083 & 4248.33 & 7.5833 \\
\hline 3658.33 & 7.1500 & 3776.67 & 7.3417 & 3835.00 & 7.4917 & 4013.33 & 7.4958 & 4131.67 & 7.5042 & 4250.00 & 7.5958 \\
\hline 3660.00 & 7.1458 & 3778.33 & 7.3542 & 3896,67 & 7.4958 & 4015.00 & 7.4958 & 4133,33 & 7.5000 & 4251,67 & 7,6000 \\
\hline 3661.67 & 7.1458 & 3780.00 & 7.3583 & 3898.33 & 7.5000 & 4016.67 & 7.4958 & 4135.00 & 7.5042 & 4253.33 & 7.6125 \\
\hline 3663.33 & 7.1417 & 3781.67 & 7.3583 & 3900.00 & 7.5083 & 4018.33 & 7.4958 & 4136.67 & 7.5000 & 4255.00 & 7.6208 \\
\hline 3665.00 & 7.1458 & 3783.33 & 7.3542 & 3901.67 & 7,5042 & 4020.00 & 7.4958 & 4138.33 & 7.5000 & 4256.67 & 7.6333 \\
\hline 3666.67 & 7.1458 & 3785.00 & 7.3583 & 3003.33 & 7.5042 & 4021.67 & 7.5000 & 4140.00 & 7.5000 & 4258.33 & 7.62 .92 \\
\hline 3668.33 & 7.1500 & 3786.67 & 7.3625 & 3905.00 & 7.4958 & 4023.33 & 7.5000 & 4141.67 & 7.4958 & 4280.00 & 7.6292 \\
\hline 3670.00 & 7.1500 & 3788.33 & 7.3667 & 3906.67 & 7.5000 & 4025.00 & 7.5000 & 4143,33 & 7.4917 & 4261.67 & 7.6375 \\
\hline 3671.67 & $\overline{7.1542}$ & 3790.00 & 7.3708 & 3008.33 & 7.4958 & 4026.67 & 7.5042 & 4945.00 & 7.4997 & 4263.33 & 7.6417 \\
\hline 3673.33 & 7.1542 & 3791.67 & 7.3708 & 3910.00 & 7.4958 & 4028.33 & 7.5083 & 4146.67 & 7.4833 & 4265,00 & 7.6500 \\
\hline 3675.00 & 7.1500 & 3793.33 & 7.3708 & 3911.67. & 7.4917 & 4030.00 & 7.5125 & 4148.33 & 7.4875 & 4266.67 & 7.6417 \\
\hline $\begin{array}{l}3676.67 \\
\end{array}$ & 7.1458 & 3795.00 & 7.3750 & 3913.33 & 7.4958 & 4031.67 & 7.5167 & 4150.00 & 7.4833 & 8.33 & 7.6500 \\
\hline 3678.33 & 7.1417 & 3796.67 & 7.3792 & 3915.00 & 7.4917 & 4033,33 & 7.5167 & 4151.67 & 7.4958 & 4270.00 & 7.6417 \\
\hline 3680.00 & 7.1458 & 3798.33 & 7.3833 & 3916.67 & 7.5000 & 4035.00 & 7.5167 & 4153.33 & 7.4917 & 4271.67 & 7.6458 \\
\hline 3681.67 & 7.1458 & 3800.00 & 7.3792 & 3918.33 & 7.5000 & 4036.67 & 7.5125 & 4155.00 & 7.4917 & 4273.33 & 7.6458 \\
\hline $3683: 33$ & 7.1458 & 3801.67 & 7.3875 & 3920.00 & 7.5042 & 4038.33 & 7.5208 & 4156,67 & 7.4833 & 4275.00 & 7.6583 \\
\hline 3685.00 & 7.1458 & 3803.33 & 7.3917 & 3921.67 & 7,5000 & 4040.00 & 7.5292 & 4158.33 & 7.4750 & 4276.67 & 7.6792 \\
\hline 3636.67 & 7.1542 & 3805.00 & 7.3958 & 3923.33 & 7.5 & 4041.67 & 7.5375 & & 7.4750 & 4278,33 & 7.6833 \\
\hline 3688.33 & 7.1583 & 3806.67 & 7.3917 & 3925.00 & 7.5125 & 4043.33 & 7.5292 & 4161.67 & 7.4792 & 4280.00 & 7.6833 \\
\hline 3690.00 & 7.1625 & 3808.33 & 7.3917 & 3926.67 & 7.5125 & 4045.00 & 7.5208 & & 7.4750 & & 7.6750 \\
\hline 3691.67 & 7.1500 & 3810.00 & 7.3875 & 3928.33 & 7.5167 & 4046.67 & 7.5125 & 4165.00 & 7.4750 & 4283.33 & 7.6708 \\
\hline 3693.33 & 7.1542 & 3811.67 & 7.3875 & 3930.00 & 7.5167 & 4048.33 & 7.5125 & & 7.4750 & 4285.00 & 7.6667 \\
\hline 3595.00 & 7.1500 & 3813.33 & 7.3875 & 3931.67 & 7.5250 & 4050.00 & 7.5208 & 4168.33 & 7.4917 & 4286.67 & 7.6208 \\
\hline 3696.67 & 7.1542 & 3815.00 & 7.3917 & 3933.33 & .7 .5250 & 4051.67 & 7.5250 & & 7.4958 & 33 & 7.5083 \\
\hline 3698.33 & 7.1458 & 3816.67 & 7.4042 & 3935.00 & 7.5208 & 4053.33 & 7.5208 & 4171.67 & 7.5000 & 4200,00 & 7.4042 \\
\hline 3700.00 & 7.1500 & 3818.33 & 7.4083 & 3936.67 & & 405 & & & 7.5000 & 67 & 7.3542 \\
\hline 3701.67 & 7.15 & 3820.00 & 7.4167 & 3998.33 & 7.5083 & 4056.67 & 7.5042 & 4175.00 & 7.5000 & 4293.33 & 7.3833 \\
\hline 3703.33 & 7.1583 & 3821.67 & 7.4167 & 3940.00 & & 4058.33 & & & 7.5042 & 4295.00 & 7.4042 \\
\hline 3705.00 & 7.1542 & 3823.33 & 7.4167 & 3941,67 & 7.5208 & 4060.00 & 7.5042 & 4178,33 & 7.5000 & 4296.67 & $7.4+67$ \\
\hline 3706.67 & 7.1542 & 3825.00 & 7.4083 & 3943.33 & 7.5250 & 4061.67 & & & 7.4958 & 4298.33 & 7.4292 \\
\hline 3708.33 & 7.1542 & 3826.67 & 7.4000 & 3945.00 & 7.5167 & 4063.33 & 7.5042 & 4181.67 & 7.4917 & .00 & 7.4375 \\
\hline 3710.00 & 7.1542 & 382 & 7.4000 & 3946.67 & 7.5125 & 4065.00 & 7.5000 & 4183.23 & 7.4958 & 4301.67 & 7.4458 \\
\hline 3711.67 & 7.1583 & 3630.00 & 7.4042 & 3948.33 & 7.5042 & 4066.67 & 7.5000 & & 7.4958 & 4303.33 & 7.4500 \\
\hline 3713.33 & 7.1542 & 3831.67 & 7.4083 & 3950.00 & 7.5000 & 4068,33 & 7.508 & 4486.67 & 7.4917 & 4305.00 & 7.4667 \\
\hline 3715.00 & 7.1542 & 3833.33 & 7.4167 & 3951.67 & 7.5000 & 4070.00 & 7.5083 & 4188.33 & 7.4917 & 4306.67 & 7.4792 \\
\hline $\begin{array}{l}3716.67 \\
\end{array}$ & 7.1500 & 3835.00 & 7.4208 & 3953.33 & 7.4958 & 4071.67 & 7.5125 & 4490.00 & 7.4917 & 4308.33 & 7.4792 \\
\hline 3718.33 & 7.1500 & 3836.67 & 7.4208 & 3955.00 & 7.5083 & 4073.33 & 7.5083 & 4191.67 & 7.4917 & 4310.00 & 7.4750 \\
\hline 3720.00 & 7.1458 & 3838.33 & 7.4208 & 3956.67 & 7.5042 & 4075.00 & 7.5167 & 4193.33 & 7.4792 & 4311.67 & 7.4708 \\
\hline 3721.67 & 7.1458 & 3840,00 & 7.4292 & 3958.33 & 7.5125 & 4076.67 & 7.5208 & 4195.00 & 7.4833 & 4313.33 & 7.4708 \\
\hline 3723.33 & 7.1500 & 3841.67 & 7.4417 & 3960.00 & 7.5042 & 4078.33 & 7.5292 & 4196.67 & 7.4750 & 4315.00 & 7.4750 \\
\hline 3725.00 & 7.1583 & 3843.33 & 7.4417 & 3961.67 & 7.5042 & 4080.00 & 7.5292 & 4198.33 & 7.4875 & 4316.67 & 7.4792 \\
\hline 3726.67 & 7.1625 & 3845.00 & 7.4417 & 3963.33 & 7.5000 & 4081.67 & 7.5333 & 4200.00 & 7.4750 & 4318.33 & 7.4875 \\
\hline 3728.33 & 7.1667 & 3846.67 & 7.4417 & 3965.00 & 7.5000 & 4083.33 & 7.5292 & 4201.67 & 7.4833 & 4320.00 & 7.4667 \\
\hline 3730.00 & 7.1750 & 3848.33 & 7.4417 & 3966.67 & 7.5000 & 4085.00 & 7.5208 & 4203.33 & 7.4750 & 4321.67 & 7.4500 \\
\hline
\end{tabular}


HNF-SD-SNF-CN-035, Rev. 0

Table C16. Thermo-Gravimetric Analysis Data for K Basin Fuel Sample Run 51 at $69^{\circ} \mathrm{C}$. (Page 7 of 7 )

\begin{tabular}{|c|c|c|c|c|c|}
\hline \multicolumn{6}{|c|}{ RUN NUMBER 61 AT 69 C (cont.) } \\
\hline $\begin{array}{l}\text { TIME } \\
\text { (minutes) }\end{array}$ & $\begin{array}{c}\text { wit change } \\
\text { (mg) }\end{array}$ & $\begin{array}{l}\text { TiME } \\
\text { (minutes) }\end{array}$ & $\begin{array}{c}\text { Wh change } \\
\text { (mg) }\end{array}$ & 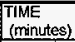 & $\begin{array}{c}\text { wh change } \\
\text { (mg) }\end{array}$ \\
\hline 4323.33 & 7.4417 & 4441.67 & 7.8625 & 4560.00 & 7.6958 \\
\hline 4325.00 & 7.4625 & 4443.33 & 7.8708 & 4561.67 & 7.6958 \\
\hline 4326.67 & 7.4792 & 4445.00 & 7.8667 & 4563.33 & 7.7042 \\
\hline 4328.33 & 7.4917 & 4446.67 & 7.8750 & 4565.00 & 7.7167 \\
\hline 4330.00 & 7.4917 & 4448.33 & 7.8708 & 4566.67 & 7.7167 \\
\hline 4331.67 & 7.4917 & 4450.00 & 7.8917 & 4568.33 & 7.7208 \\
\hline 4333.33 & 7.4917 & 4451.67 & 7.9042 & 4570.00 & 7.7208 \\
\hline 4335.00 & 7.4917 & 4453.33 & 7.9000 & 4571.67 & 7.7250 \\
\hline 4336.67 & 7.4958 & 4455.00 & 7.8958 & 4573.33 & 7.7333 \\
\hline 4338.33 & 7.5083 & 4456.67 & 7.8958 & 4575.00 & 7.7458 \\
\hline 4340.00 & 7,5208 & 4458.33 & 7.9000 & 4576.67 & 7.7542 \\
\hline 4341.67 & 7.5208 & 446000 & 7.8917 & 4578.33 & 7.7500 \\
\hline 4343.33 & 7.5167 & $4461: 67$ & 7.8792 & 4580.00 & 7.7417 \\
\hline 4345.00 & 7.5167 & 4463.33 & 7.8917 & 4581.67 & 7.7458 \\
\hline 4346.67 & 7.5333 & 4465.00 & 7.9042 & 4583.33 & 7.7542 \\
\hline 4348.33 & 7.5458 & 4466.67 & 7.9167 & 4585.00 & 7.7583 \\
\hline 4350.00 & 7.5458 & 4468.33 & 7.9167 & 4586.67 & 7.7625 \\
\hline 4351.67 & 7.5500 & 4470.00 & 7.9167 & 4588.33 & 7.7708 \\
\hline 4353.33 & 7.5583 & 4471.67 & 7.9167 & 4590.00 & 7.7750 \\
\hline 4355.00 & 7,5708 & 4473.33 & 7.9125 & 4591.67 & 7.7875 \\
\hline 4356.67 & 7.5792 & 4475.00 & 7.9083 & 4593.33 & 7.8042 \\
\hline 435.8 .33 & 7.5792 & 4476.67 & 7.9083 & 4595.00 & 7.8292 \\
\hline 4360.00 & 7,5792 & 4478.33 & 7.9083 & 4596.67 & 7.8375 \\
\hline 4361,67 & 7.5708 & 4480.00 & 7.9167 & 4598.33 & 7.8375 \\
\hline 4363.33 & 7.5667 & 4481.67 & 7.9250 & 4600.00 & 7.8500 \\
\hline 4365.00 & 7.5750 & 4483.33 & 7.9333 & 4601.67 & 7.8542 \\
\hline 4366.67 & 7.5750 & 4485.00 & 7.9292 & 4603.33 & 7.8583 \\
\hline 4368.33 & 7,5833 & 4486.67 & 7.9292 & 4605.00 & 7.8583 \\
\hline 4370.00 & 7.5958 & 4488.33 & 7.9333 & 4606.67 & 7.8833 \\
\hline 4371.67 & 7.6083 & 4490.00 & 7.9417 & 4608.33 & 7.9042 \\
\hline 4373.33 & 7.6167 & 4491.67 & 7.9417 & 4610.00 & 7.9292 \\
\hline 4375.00 & 7.6208 & 4493.33 & 7.9333 & 4611.67 & 7.0458 \\
\hline 4376.67 & 7.6458 & 4495.00 & 7.9333 & 4613.33 & 7.9583 \\
\hline 4378.33 & 7.6583 & 4496.67 & 7.9458 & 4615.00 & 7.9657 \\
\hline 4380.00 & 7.6525 & 4498.33 & 7.9600 & 4616.67 & 7.9500 \\
\hline 4381.67 & 7.6583 & 4500.00 & 7.9500 & 4618.33 & 7.9583 \\
\hline 4383.33 & 7,6708 & 4501.67 & 7.9292 & 4620.00 & 7.9583 \\
\hline 4335.00 & 7.6750 & 4503.33 & 7.9292 & 4621.67 & 7.9833 \\
\hline 4386.67 & 7.6750 & 4505.00 & 7.9292 & 4623.33 & 7.9875 \\
\hline 4388.33 & 7.6750 & 4506.67 & 7.9375 & 4625.00 & 8.0042 \\
\hline 4390.00 & 7.6958 & 4508.33 & 7.9292 & 4626.67 & 8.0208 \\
\hline 4391.67 & 7.7167 & 4510.00 & 7.9167 & 4628.33 & 8.0333 \\
\hline 4393.33 & 7.7167 & 4511.67 & 7.0000 & 4630.00 & 8.0333 \\
\hline 4395.00 & 7.7208 & 4513.33 & 7.8958 & 4631.67 & 8.0250 \\
\hline 4396.67 & 7.7208 & 4515.00 & 7.9000 & 4633.33 & 8.0333 \\
\hline 4398.33 & 7.7208 & 4516.67 & 7.9000 & 4635.00 & 8.0417 \\
\hline 4400.00 & 7.7292 & 4518.33 & 7.8833 & 4536.67 & 8.0583 \\
\hline 4401.67 & 7.7333 & 4520.00 & 7.8708 & 4638.33 & 8.0708 \\
\hline 4403.33 & 7.7458 & 4521.67 & 7.8542 & 4640.00 & 8.0833 \\
\hline 4405.00 & 7.7375 & 4523.33 & 7.8458 & 4641.67 & 8.0875 \\
\hline 4406.67 & 7.7458 & 4525,00 & 7.8250 & & \\
\hline 4408.33 & 7.7625 & 4526.67 & 7.8167 & & \\
\hline 4410.00 & 7.7708 & 4528.33 & 7.8083 & & \\
\hline 4411.67 & 7.7876 & 4530.00 & 7.8042 & & \\
\hline 4413.33 & 7.7833 & 4531.67 & 7.7875 & & \\
\hline 4415.00 & 7.7875 & 4533.33 & 7.7708 & & \\
\hline 4416.67 & 7.7792 & 4535.00 & 7.7500 & & \\
\hline 4418.33 & 7.7792 & 4536.67 & 7.7417 & & \\
\hline 4420.00 & 7.7917 & 4538.33 & 7.7333 & & \\
\hline 4421.67 & 7.7917 & 4540.00 & 7.7208 & & \\
\hline 4423.33 & 7.8042 & 4541,67 & 7.7208 & & \\
\hline 4425.00 & 7.8042 & 4543.33 & 7.7125 & & \\
\hline 4426.67 & 7.8167 & 4545.00 & 7.7083 & & \\
\hline 4428.33 & 7.8250 & 4546.67 & 7.6917 & & \\
\hline 4430.00 & 7.8333 & 4548.33 & 7.6917 & & \\
\hline 4431.67 & 7.8375 & 4550.00 & 7.6958 & & \\
\hline 4433.33 & 7.8375 & 4551.67 & 7.7042 & & \\
\hline 4435.00 & 7.8375 & 4553.33 & 7.7042 & & \\
\hline 4436.67 & 7.8458 & 4555.00 & 7.7083 & & \\
\hline 4436.33 & 7.8583 & 4556.67 & 7.7083 & & \\
\hline 4440.00 & 7.8667 & 4558.33 & 7.7042 & & \\
\hline
\end{tabular}




\section{DISTRIBUTION SHEET}

\begin{tabular}{|c|c|c|c|c|c|}
\hline To & \multirow{2}{*}{\multicolumn{3}{|c|}{$\begin{array}{l}\text { From } \\
\text { SNF Characterization } \\
\text { Project/2T720 }\end{array}$}} & \multicolumn{2}{|l|}{ Page 1 of 2} \\
\hline Distribution & & & & \multicolumn{2}{|c|}{ Date February 1998} \\
\hline \multicolumn{4}{|l|}{ Project Title/Work Order } & \multicolumn{2}{|l|}{ EDT No. } \\
\hline HNF-SD-SNF-CN-035, Rev. 1 & & & & \multicolumn{2}{|c|}{ ECN No. 614814} \\
\hline Name & MSIN & $\begin{array}{l}\text { Text } \\
\text { With All } \\
\text { Attach. }\end{array}$ & Text Only & $\begin{array}{l}\text { Attach./ } \\
\text { Appendix } \\
\text { Only }\end{array}$ & $\begin{array}{c}\text { EDT/ECN } \\
\text { Only }\end{array}$ \\
\hline
\end{tabular}

Fluor Daniel Hanford, Inc.

E. W. Gerber

R3-11 X

\section{ARES}

J. P. Sederburg

R3-86

$x$

B\&W Protec, Inc.

T. L. We1sh

$\mathrm{T} 4-40$

$x$

Duke Engineering \& Services Hanford, Inc.

R. B. Baker

D. W. Bergmann

D. R. Duncan

J. R. Frederickson

W. M. Funderburke

L. A. Lawrence

P. G. LeRoy

B. J. Makenas

R. P. Omberg

W. H. Rasin

A. M. Segrest

D. W. Smith

J. A. Swenson

D. J. Trimble (5)

SNF Project Files

Dyncorp Tri-Cities Services, Inc.
HO- 40

X3-79

R3-86

R3-86

R3-11

HO- 40

R3-26

$\mathrm{HO}-40$

$\mathrm{HO}-40$

R3-11

R3-13

R3-26

R3-11

Ho- 40

R3-11

R3-86

$x$

Fluor Daniel Northwest

R. D. Crowe

F. F. Huang
R3-26

E6-15
$X$
$X$
$X$
$X$
$X$
$X$
$X$
$X$
$X$
$X$
$X$
$X$
$X$
$X$
$X$ 


\section{DISTRIBUTION SHEET}

\begin{tabular}{|c|c|c|c|c|c|}
\hline To & \multirow{2}{*}{\multicolumn{3}{|c|}{$\begin{array}{l}\text { From } \\
\text { SNF Characterization } \\
\text { Project/2T720 }\end{array}$}} & \multicolumn{2}{|l|}{ Page 2 of 2} \\
\hline Distribution & & & & \multicolumn{2}{|c|}{ Date February 1998} \\
\hline \multirow{2}{*}{\multicolumn{4}{|c|}{ Project Title/Work Order }} & \multicolumn{2}{|l|}{ EDT No. } \\
\hline & & & & \multicolumn{2}{|c|}{ ECN No. 614814} \\
\hline Name & MSIN & $\begin{array}{l}\text { Text } \\
\text { With All } \\
\text { Attach. }\end{array}$ & Text Only & $\begin{array}{l}\text { Attach./ } \\
\text { Appendix } \\
\text { Only }\end{array}$ & $\begin{array}{l}\text { EDT/ECN } \\
\text { Only }\end{array}$ \\
\hline
\end{tabular}

Lockheed Martin Hanford Corp.

J. H. Wicks, Jr.

R3-11 X

Lockheed Martin Services. Inc.

Central Files

BT-07 $\quad X$

Numatec Hanford Corporation

E. R. Cramer

T. A. Flament

J. J. Irwin

C. R. Miska

J. P. Sloughter

HO-34

R3-86

R3-86

R3-86

H5-49

$x$
$X$
$x$
$x$
$X$

Pacific Northwest National Laboratory

J. Abrefah

J. M. Latkovich

P7-27

K9-44

P7-27

$x$
$x$
$x$

U.S. Department of Energy,

Richland Operations office
P. G. Loscoe
E. D. Sellers
$J$. Shuen

$\begin{array}{ll}S 7-41 & x \\ 57-41 & X \\ S 7-41 & x\end{array}$ 